\title{
Bioinspired Investigation via X-Ray Microtomography
}

\author{
North, Laura E.
}

How to cite:

North, Laura E. (2018) Bioinspired Investigation via X-Ray Microtomography. Doctoral thesis, Swansea University. http://cronfa.swan.ac.uk/Record/cronfa43706

Use policy:

This item is brought to you by Swansea University. Any person downloading material is agreeing to abide by the terms of the repository licence: copies of full text items may be used or reproduced in any format or medium, without prior permission for personal research or study, educational or non-commercial purposes only. The copyright for any work remains with the original author unless otherwise specified. The full-text must not be sold in any format or medium without the formal permission of the copyright holder. Permission for multiple reproductions should be obtained from the original author.

Authors are personally responsible for adhering to copyright and publisher restrictions when uploading content to the repository.

Please link to the metadata record in the Swansea University repository, Cronfa (link given in the citation reference above.)

http://www.swansea.ac.uk/library/researchsupport/ris-support/ 


\title{
Bioinspired Investigation via X-Ray Microtomography
}

\author{
by \\ Laura Elizabeth North, MSc, BEng \\ Thesis submitted to Swansea University \\ in fulfilment of the requirements for the Degree of \\ Doctor of Philosophy
}
College of Engineering
Swansea University
Swansea SA2 8EN
United Kingdom

March 2018 


\section{Summary}

Biological materials and systems are increasingly studied to provide inspiration, through the correlation of structure and function, for the design of materials in areas such as technology, engineering and medicine. X-ray microtomography allows three dimensional and non-destructive visualisation of both internal and external structures. It is the primary method used in this study to identify and investigate these natural structures and their functions. Both quantitative and qualitative analysis is performed on the resulting $3 \mathrm{D}$ volumetric data. Further insight is achieved by incorporating complementary methods including high-resolution electron microscopy, nanoindentation and additive layer manufacturing to characterise the structures at varying length scales in terms of their structural, chemical and mechanical properties. Two detailed case studies are given: the vertebrae of the hero shrew (Scutisorex somereni); and the cuttlebone of Sepia officinalis.

Hero shrew vertebrae are analysed for the first time using X-ray microtomography. Large variations in vertebrae volume, surface area and pillar count are shown across samples. Additive layer manufacturing is used to test a simple method for understanding flexibility across the vertebrae. The results show limitations of movement in certain directions, giving potential inspiration for applications in robotics and flexible shafts.

The diversity of internal architecture of the cuttlebone is captured for the first time in three dimensions, highlighting substantial variation in the morphology of pillars. New frameworks are established for pillar morphology across the cuttlebone. These provide a greater understanding to the relationship between pillar morphology and fluid interaction with the structures of the cuttlebone. Mechanical analysis via time-lapse compression testing shows a progressive collapse mechanism of the chambers. The morphology and properties investigated can provide inspiration for improved design of cellular structures, energy absorption and protection, and potentially for the design of a sophisticated buoyancy device. 


\section{Declaration}

This work has not previously been accepted in substance for any degree and is not being concurrently submitted in candidature for any degree.

\section{LAURA NORTH \\ $13^{\text {th }}$ March 2018}

\section{Statement}

This thesis is the result of my own investigations, except where otherwise stated and other sources are acknowledged by explicit references and a bibliography is appended.

\section{LAURA NORTH \\ $13^{\text {th }}$ March 2018}

\section{Statement}

I hereby give consent for my thesis, if accepted, to be available for photocopying and for inter-library loan (subject to the law of copyright), and for the title and summary to be made available to outside organisations.

LAURA NORTH

$13^{\text {th }}$ March 2018 


\section{Acknowledgements}

I would firstly like to thank my ace supervisor Dr Richard Johnston for his invaluable support, enthusiasm, ideas and patience.

I have received a huge amount of support from everyone in the JohnstonLab and AIM facility at Swansea University, especially Dr Mark Coleman, Dr Dan Moyle and Dr Ross Buckingham. A massive thank you to you all. Many thanks also to Pete Davies for all his superb technical support. I will miss all those cups of tea.

Thanks go out to all the people I have been fortunate enough to collaborate with, in particular: Prof Michelle Oyen, Dr David Labonte, Dr James McGettrick and Dr Ed Pope.

Biggest thanks of all goes to my PIC Pat, and my family for all the encouragement, support, advice and proof reading.

Finally, I would like to thank the Engineering and Physical Sciences Research Council (EPSRC) for funding this project.

LAURA NORTH

Swansea University

March 2018 


\section{Contents}

1 Introduction 1

2 Review of Literature 3

2.1 Biological materials science . . . . . . . . . . . . . . . . 3

2.1.1 Fundamentals . . . . . . . . . . . . . . . . . . . . 4

2.1.2 Structural design elements . . . . . . . . . . . . . . . . . . . 6

2.1.3 Biological materials . . . . . . . . . . . . . . . . . . 8

2.1.4 Bioinspired materials and biomimetics . . . . . . . . . . . . 12

2.2 Imaging techniques . . . . . . . . . . . . . . . . . . . . . . . 14

2.2.1 X-ray Computed Tomography . . . . . . . . . . . . . . . . . . 14

2.3 X-ray microtomography in biological materials science . . . . . . . . . . . . . 19

2.3.1 Sample preparation . . . . . . . . . . . . . . . . 20

2.3.2 Bioinvestigation from functional designs . . . . . . . . . . . . 21

$2.3 .34 \mathrm{D}$ imaging . . . . . . . . . . . . . . . . . 26

2.3.4 Additive layer manufacturing . . . . . . . . . . . . . . 28

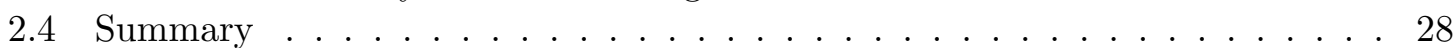

3 Core Methodologies $\quad 30$

3.1 X-ray microtomography . . . . . . . . . . . . . . . . . . . . 30

3.1 .1 Systems . . . . . . . . . . . . . . . . . . 30

3.1.2 Optimisation of CT scanning . . . . . . . . . . . . . 31

3.1.3 Reconstruction and data visualisation . . . . . . . . . . . . . 32

3.2 Scanning Electron Microscopy . . . . . . . . . . . . . . . . . . . . . . 34

3.3 Energy Dispersive X-ray Spectroscopy . . . . . . . . . . . . . . . . . . 34

3.4 Electron backscatter diffraction analysis . . . . . . . . . . . . . 36

3.4 .1 EBSD technique . . . . . . . . . . . . . . . 36

3.4.2 Preparation of samples for EBSD . . . . . . . . . . . . . . . 37

3.4.3 EBSD data analysis and representation . . . . . . . . . . . 37

3.5 Nanoindentation . . . . . . . . . . . . . . . . . . . . . 38

3.5.1 Mechanics of nanoindentation . . . . . . . . . . . . . . . 40

3.6 3D printing . . . . . . . . . . . . . . . . . . . . 41

4 Vertebrae of Hero Shrew 43

4.1 Introduction and previous work . . . . . . . . . . . . . . . . 43

4.2 Experimental methods . . . . . . . . . . . . . . . . . . . 46

4.2 .1 Specimens . . . . . . . . . . . . . . . . . . . 47

4.2 .2 X-ray microtomography . . . . . . . . . . . . . . . . . . 44 47

4.2 .3 Optical microscopy . . . . . . . . . . . . . . . . . . . . . . 49 
4.2.4 3D printing and vertebral range-of-movement analysis . . . . . . . . 49

4.3 Results and discussion . . . . . . . . . . . . . . . . . . . 50

4.3 .1 Vertebrae analysis . . . . . . . . . . . . . . . . 50

4.3.2 Vertebrae sample comparison . . . . . . . . . . . . . . 56

4.3 .3 Vertebra interaction . . . . . . . . . . . . . . 63

4.3 .4 Discussion . . . . . . . . . . . . . . . . . . . . 64

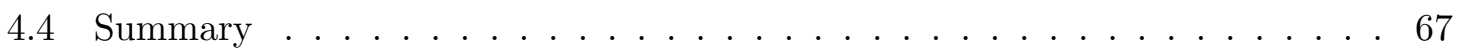

5 Cuttlebone of Sepia officinalis $\quad 68$

5.1 Introduction and previous work . . . . . . . . . . . . . . . . 68

5.2 Experimental methods . . . . . . . . . . . . . . . . . . . . . 72

5.2 .1 Specimens . . . . . . . . . . . . . . . . 72

5.2 .2 Structural characterisation . . . . . . . . . . . . . 73

5.2 .3 Chemical analysis . . . . . . . . . . . . . . . . 85

5.2 .4 Mechanical testing . . . . . . . . . . . . . . . . . 87

5.3 Results and discussion . . . . . . . . . . . . . . . . . . . . . 90

5.3 .1 Structural analysis . . . . . . . . . . . . . . . . . 9 91

5.3 .2 Chemical analysis . . . . . . . . . . . . . . . . . 132

5.3 .3 Mechanical analysis . . . . . . . . . . . . . . . . 137

5.4 Summary . . . . . . . . . . . . . . . . . . . . . . 149

6 Conclusions $\quad 150$

7 Recommendations for Future Work $\quad \mathbf{1 5 2}$

7.1 Hero shrew . . . . . . . . . . . . . . . . . . . . . . . . . . . 152

7.2 Cuttlebone . . . . . . . . . . . . . . . . . . 153

$\begin{array}{ll}\text { A SolidWorks Sketches } & 154\end{array}$

$\begin{array}{lr}\text { B Publications } & 156\end{array}$

$\begin{array}{ll}\text { Bibliography } & 164\end{array}$ 


\section{List of Figures}

2.1 Eight principal biological structural design elements . . . . . . . . . . 6

2.2 Ashby plot for biological materials showing strength as a function of density. 9

2.3 Ashby plot for biological materials showing toughness as a function of modulus. 10

2.4 The hierarchical structure of bone and bamboo. . . . . . . . . . . . . . 11

2.5 Schematic of an X-ray tube and the photon energy distribution of bremsstrahlung and characteristic X-rays . . . . . . . . . . . . 15

2.6 Sketch of projection acquisition for a cone-beam CT system . . . . . . . . . 17

2.7 Illustration of the Fourier slice theorem. . . . . . . . . . . . . . . 18

2.8 Illustration of the reconstruction process using filtered back-projection. . . . . 19

2.9 Examples of artefacts in X-ray $\mu \mathrm{CT} \ldots \ldots \ldots \ldots$

2.10 Effects of staining in absorption contrast X-ray $\mu \mathrm{CT} \ldots \ldots \ldots . . . . .21$

2.11 Conventional absorption contrast tomography and phase contrast tomography 22

$2.12 \mu \mathrm{CT}$ images of fifth abdominal tergite and the telson . . . . . . . . . . 23

2.13 High-resolution $\mu \mathrm{CT}$ images of a boxfish scute. . . . . . . . . . . . . . 24

2.14 Seahorse tail cross-sections and $\mu \mathrm{CT}$ images. . . . . . . . . . . . 25

2.15 Seahorse tail sections under compressive load. . . . . . . . . . . . . 26

$2.163 \mathrm{D}$ reconstructed $\mu \mathrm{CT}$ model of a denticle of a mako shark (Isurus oxyrinchus) 27

$2.173 \mathrm{D}$ printed prototypes designed to mimic the specialised joints of seahorse tail. 28

3.1 Schematic of a conventional $\mu \mathrm{CT}$ and optics based $\mu \mathrm{CT}$ system. . . . . . . 31

3.2 Examples of difficulties encountered when CT scanning. . . . . . . . . . . . 33

3.3 Representative example of EDS elemental mapping and the spectrum of elements identified . . . . . . . . . . . . . . . . . . 35

3.4 Set-up for EBSD analysis . . . . . . . . . . . . . . . . . 36

3.5 Inverse pole figure (IPF) crystal model and colour key for aragonite. . . . . . 38

3.6 Representation of EBSD data as a stereographic pole plot. . . . . . . . . . . 38

3.7 Illustration of a nanoindentation measurement . . . . . . . . . . . . . . . 39

3.8 Schematic of a typical load-displacement curve . . . . . . . . . . . . . . . 39

3.9 An example of the process of fabricating 3D printed structures . . . . . . . . 41

4.1 Sketch of Scutisorex somereni and its skeleton. . . . . . . . . . . . . . . . 43

4.2 Lateral views of lumbar region, and thoracic region, of African giant shrew (Crocidura olivieri), hero shrew (Scutisorex somereni), and Thor's hero shrew (Scutisorex thori). . . . . . . . . . . . . . . . . . 44

4.3 Lateral and dorsal views of hero shrew spine with anatomical directions. . . . 45

4.4 Hero shrew specimens provided by Dr. Cullinane. . . . . . . . . . . . . . . 47

4.5 Segmented vertebrae used for analysis. . . . . . . . . . . . . . 51

4.6 Cranial view of vertebra with labels indicating features. . . . . . . . . . 51

4.7 Vertebra volume. . . . . . . . . . . . . . . . . 53 
4.8 Vertebra surface area. . . . . . . . . . . . . . . . . . . . 54

4.9 Tubercle count for each vertebra. . . . . . . . . . . . . . . . . . 55

4.10 Tubercle count differences between the vertebra cranial face and the preceding vertebra caudal face. . . . . . . . . . . . . . . . 5 55

$4.113 \mathrm{D}$ renders of adjacent vertebrae $\mathrm{L} 5$ and $\mathrm{L} 6 \ldots \ldots \ldots \ldots$

$4.123 \mathrm{D}$ renders of vertebrae from re-articulated skeleton specimen. . . . . . . . 57

4.13 Internal structure of the hero shrew vertebrae. . . . . . . . . . . . . . 57

$4.143 \mathrm{D}$ renders of vertebrae virtually cropped to reveal internal structures. . . . . 58

4.15 Vertebra volume of common vertebrae on three samples. . . . . . . . . . . . 60

4.16 Vertebra surface area of common vertebrae on three samples. . . . . . . . . . 60

4.17 Tubercle counts of common vertebrae on three samples. . . . . . . . . . . . 61

4.18 3D renders of vertebrae from re-articulated skeleton and US samples. . . . . . 62

4.19 Scatterplots of volume vs surface area, volume vs tubercle count and surface area vs tubercle count. . . . . . . . . . . . . . . . . . . 62

$4.20 \mathrm{~T} 8$ - L2 tubercle count differences between the vertebra cranial face and the preceding vertebra caudal face. . . . . . . . . . . . . . 63

4.21 Dorsal and lateral views of 3D prints of vertebrae L4 - L6 at maximum displacement in the lateral flexion, extension and flexion directions. . . . . . . 65

4.22 Dorsal and lateral radiographs of the skeleton of Scutisorex somereni. . . . . 66

5.1 Schematic of a cuttlebone from Sepia officinalis. . . . . . . . . . . . . . 69

5.2 SEM image of transverse section of cuttlebone sample and unit cell model extracted from SEM images. . . . . . . . . . . . . . . . . . 71

5.3 Representation of the principal axes for preparation of the cuttlebone. . . . . 72

5.4 Schematic of cuttlebone cores used for morphological analysis. . . . . . . . . . 75

5.5 Longitudinal and transverse axes with percentages used to reference core

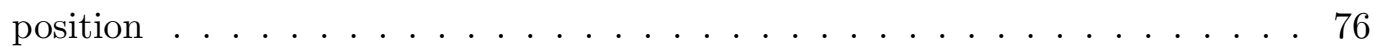

5.6 Comparison of different cuttlebone samples showing regions of similar pillar morphology. . . . . . . . . . . . . . . . . . 77

5.7 Five pillars from core_4c - chamber 10 - slice 40 used to validate measurements of pillars. . . . . . . . . . . . . . . . . . . 79

5.8 The major steps for preparing slices for analysis in ImageJ. . . . . . . . . . 80

5.9 Pillar 9 from core_4c - chamber 10, displaying measurements acquired using ImageJ. . . . . . . . . . . . . . . . . . . . . . 81

5.10 Examples of slices removed from analysis. . . . . . . . . . . . . . 82

5.11 Flow chart of iterative process used to produce EBSD maps of cuttlebone. . . 84

5.12 XPS schematic. . . . . . . . . . . . . . . . . . 86

5.13 XPS wide spectrum of cuttlebone. . . . . . . . . . . . . . 87

5.14 Schematic of compression testing. . . . . . . . . . . . . . . . 88

5.15 Example of area for nanoindentation. . . . . . . . . . . . . . . . . 89

5.16 Cross-section at mid-point of single chamber STL files used in compression analysis. . . . . . . . . . . . . . . . . . . 90

5.17 Comparison of number of slices acquired for each core of cuttlebone with the number of slices used for analysis. . . . . . . . . . . . . . . . . . 94

5.18 Slices of cuttlebone taken from chamber 10 in core_4c, showing pillar morphology across different sections of the chamber. . . . . . . . . . . . . 96

5.19 Pillar counts recorded by distance from chamber roof for each chamber in core_1b. . . . . . . . . . . . . . . . . . . 97 
5.20 Pillar counts recorded by distance from chamber roof for each chamber in core_2a. . . . . . . . . . . . . . . . . . . 97

5.21 Pillar counts recorded by distance from chamber roof for each chamber in core_2b. . . . . . . . . . . . . . . . . . . . 98

5.22 Pillar counts recorded by distance from chamber roof for each chamber in core_3c. . . . . . . . . . . . . . . . . . . . 98

5.23 Pillar counts recorded by distance from chamber roof for each chamber in

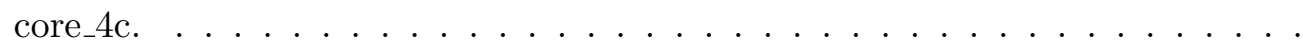

5.24 Pillar counts recorded by distance from chamber roof for each chamber in core_5c. . . . . . . . . . . . . . . . .

5.25 Pillar counts recorded by distance from chamber roof for each chamber in core_6b. . . . . . . . . . . . . . . . . . . 100

5.26 Sliced CT data of core_4c - chamber 32. . . . . . . . . . . . . . . 101

5.27 Overlaid slices taken from core_4c showing pillar alignment. . . . . . . . . . . 102

5.28 Material-to-air percentage recorded by distance from chamber roof for each chamber in core_1b. . . . . . . . . . . . . . . . . . . . . . . 103

5.29 Material-to-air percentage recorded by distance from chamber roof for each chamber in core_2a. . . . . . . . . . . . . . . . . . . . . 103

5.30 Material-to-air percentage recorded by distance from chamber roof for each chamber in core_2b. . . . . . . . . . . . . . . . . . . . . . . 104

5.31 Material-to-air percentage recorded by distance from chamber roof for each chamber in core_3c. . . . . . . . . . . . . . . . . . . . . . . . 104

5.32 Material-to-air percentage recorded by distance from chamber roof for each chamber in core_4c. . . . . . . . . . . . . . . . . . . . . 105

5.33 Material-to-air percentage recorded by distance from chamber roof for each chamber in core_5c. . . . . . . . . . . . . . . . . . . . . 105

5.34 Material-to-air percentage recorded by distance from chamber roof for each chamber in core_6b. . . . . . . . . . . . . . . . . . . 106

5.35 A boxplot showing the distribution of pillar perimeters for six slices taken from chamber 10 of core $4 . \ldots 107$

5.36 A boxplot showing the distribution of pillar Feret lengths for six slices taken from chamber 10 of core $4 . \ldots \ldots 107$

5.37 A boxplot showing the distribution of pillar tortuosity for six slices taken from chamber 10 of core 4 . . . . . . . . . . . . . . . . . . . 108

5.38 Average tortuosity recorded by distance from chamber roof for each chamber in core_1b. . . . . . . . . . . . . . . . . . . . . . 108

5.39 Average tortuosity recorded by distance from chamber roof for each chamber in core_2a. . . . . . . . . . . . . . . . . . . . . . 109

5.40 Average tortuosity recorded by distance from chamber roof for each chamber in core_2b. . . . . . . . . . . . . . . . . . . . . . . . 109

5.41 Average tortuosity recorded by distance from chamber roof for each chamber in core_3c. . . . . . . . . . . . . . . . . . . . . . 110

5.42 Average tortuosity recorded by distance from chamber roof for each chamber in core 4c. . . . . . . . . . . . . . . . . . . . . . 110

5.43 Average tortuosity recorded by distance from chamber roof for each chamber in core_5c. . . . . . . . . . . . . . . . . . . . . . 111

5.44 Average tortuosity recorded by distance from chamber roof for each chamber in core_6b. . . . . . . . . . . . . . . . . . . . . . . 111 
5.45 Four slices from core_ $1 \mathrm{~b}$ - chamber 3 with pillars considered for analysis coloured red. . . . . . . . . . . . . . . . . . . . . . 113

5.46 Pillar counts, mean pillar area, material to air percentages and mean pillar perimeters for each chamber of seven sample cores with conditional formatting.114

5.47 Mean pillar Feret lengths, mean pillar tortuosity, mean pillar thickness and mean pillar spacing for each chamber of seven sample cores with conditional formatting. . . . . . . . . . . . . . . . . . . . 115

5.48 Schematic of cuttlebone indicating regions of pillar counts. . . . . . . . . . 116

5.49 Schematic of cuttlebone indicating regions of material-to-air percentage. . . . 117

5.50 Schematic of cuttlebone indicating regions of pillar tortuosity. . . . . . . . . 117

5.51 Plots showing the distribution of fluid in chambers of four cuttlebone of varying densities. . . . . . . . . . . . . . . . . . . . 119

5.52 Example from core_5c chamber 22 slice 008 of the alignment of pillars in proximity to the siphonal zone. . . . . . . . . . . . . . . . . . 120

5.53 Example from core_6b chamber 10 of pillars splitting near the floor of a chamber. 120

5.54 Examples of tree shaped pillars from (a) core_3c and (b) core_6b. . . . . . . . 121

5.55 Examples of pillar alignment. . . . . . . . . . . . . . . . . . . . 122

5.56 Two images of straight pillars. . . . . . . . . . . . . . . . . . . 123

5.57 Straight line and bifurcation patterns seen in nature. . . . . . . . . . . . . . . 124

5.58 Secondary and backscattered electron SEM images of the dorsal shield and intersection with the lamellar matrix. . . . . . . . . . . . . . . . 125

5.59 SEM images of pillars. . . . . . . . . . . . . . . . . . . . . 125

5.60 SEM images of septa. . . . . . . . . . . . . . . . . . . . 127

5.61 SEM images of a septum that has been sectioned by FIB. . . . . . . . . . . 128

5.62 Band contrast maps. . . . . . . . . . . . . . . . . . . . . . 129

5.63 Inverse pole figure (IPF) maps and pole figure plots of crystallographic orientation of a septum. . . . . . . . . . . . . . . . . . 130

5.64 Inverse pole figure (IPF) with band contrast maps and pole figure plots of crystallographic orientation of the outer dorsal shield. . . . . . . . . . . 131

5.65 EDS map of Ca distribution in the dorsal shield. . . . . . . . . . . . . . . . 133

5.66 Samples used for XPS analysis. . . . . . . . . . . . . . . . . . . 134

5.67 Elemental wide spectrum of cuttlebone. . . . . . . . . . . . . . . . . 134

5.68 2D elemental distribution from XPS analysis. . . . . . . . . . . . 136

5.69 Load-displacement curves for all stages of compressive loading. . . . . . . . . 140

$5.703 \mathrm{D}$ renders of the bulk specimen after each stage of compressive loading. . . . 141

5.71 Magnified regions of highlighted areas in Figure 5.70 . . . . . . . . . . . . . 142

5.72 Stress-strain curves for all single pillars . . . . . . . . . . . . . . . . . . . . . 144

5.73 STL cross-sections at midpoint of chambers and their corresponding stressstrain curves. . . . . . . . . . . . . . . . . . . . . 146

5.74 Before and after compression photos for cham2 (green) and cham3 (blue). . . 147

5.75 Load-displacement curves for three chamber cuttlebone structure and three layer honeycomb structure . . . . . . . . . . . . . . . . . . . 147

5.76 Before and after compression photos of three layer cuttlebone structure and three layer honeycomb structure . . . . . . . . . . . . . . . . 148

A.1 Sketch of honeycomb structure. . . . . . . . . . . . . . . . . . . 155

A.2 Sketch of filleted pillar. . . . . . . . . . . . . . . . . . . 155 


\section{List of Tables}

4.1 X-ray $\mu \mathrm{CT}$ acquisition parameters for shrew samples. . . . . . . . . . . . 48

4.2 Summary of vertebrae from re-articulated skeleton. . . . . . . . . . . . . 52

4.3 Summary of vertebrae analysis from US_sample2. . . . . . . . . . . . . . 58

4.4 Summary of vertebrae analysis from US_sample3. . . . . . . . . . . . . . 59

4.5 Angles of rotation from 3D prints of vertebrae L4 and L6 relative to L5. . . . 64

5.1 X-ray $\mu \mathrm{CT}$ acquisition parameters for cuttlebone samples. . . . . . . . . . . 74

5.2 Position of sample cores relative to the total length and width of cuttlebone. $\quad 76$

5.3 Measurements of five pillars from core_4c - chamber 10 - slice 40 at two extreme threshold levels compared to threshold level used for quantitative analysis. . . . . . . . . . . . . . . . . . . . . . 79

5.4 Imaging modes for all scanning electron microscopes used. . . . . . . . . . . 83

5.5 EBSD map acquisition settings. . . . . . . . . . . . . . . 85

5.6 Reference positions of samples taken directly from CT data . . . . . . . . . 91

5.7 STL file dimensions, 3D print settings and compression test settings . . . . . 92

5.8 A summary of the number of chambers, and the minimum and maximum complete chambers for each core. . . . . . . . . . . . . . . 93

5.9 A summary of pillar counts and average pillar measurements of six slices from chamber 10 of core_4c. . . . . . . . . . . . . . . . . . . 95

5.10 Region element composition \% acquired by XPS analysis. . . . . . . . . . 135

5.11 Reduced elastic modulus and hardness measurements obtained from nanoindentation testing. . . . . . . . . . . . . . . . . 137 


\section{Chapter 1}

\section{Introduction}

Throughout nature, biological materials and complex structures, formed by organisms, exhibit outstanding mechanical and often multi-functional properties. These natural materials and structures have unique characteristics which differentiate them from their synthetic counterparts, with evolution, environmental constraints, and the limited availability of materials dictating their properties and morphology.

These complex structures are characterised by their hierarchical organisation at the nano-, micro-, and meso-levels, and their composite designs. The basic inorganic building blocks of most natural systems are independently very weak, however in combination with organic components and organised into highly-ordered structures, these systems make composites that have better mechanical properties than their synthetic equivalents $[1,2]$. In addition to the precise, intricate and sometimes unique architectures, biological materials are self-assembled at ambient temperatures and pressures, which if fully understood could lead to significant advances in materials science and novel synthesis techniques for multi-scale composites.

There are numerous examples of biological materials exhibiting excellent mechanical properties, whilst being hierarchically organised and functional. These include: the stomatopod dactyl club, which can withstand repetitive large impact forces with minimum damage due to its complex and ordered structure [3, 4]; silica rods in sea sponge skeletons that have high flexural toughness and flexibility compared with brittle synthetic rods [5]; and the toucan beak, which combines a solid keratin external shell with a cellular core enabling the structure to have a low density and sufficient stiffness [6].

Biomimetics is an interdisciplinary field across materials science and biology, in which lessons learned from nature form the basis for the development of novel materials. The field investigates biological materials and systems, and establishes the structure-function relationships, in order to develop new designs and concepts. The understanding and principles of biological systems that have benefited from evolutionary refinement can then be applied to modern synthetic materials using advanced technology.

Motivation for this study arises from the desire to further research in the field of bio- 
mimetics by utilising modern non-destructive imaging techniques to visualise both external and internal structures within nature in order to provide insight into the structure-function relationships. The ultimate aim is to inspire design and engineering improvements as a result of the increased understanding of these relationships.

This study is focussed on two unique bioarchitectures found in nature: the spine of the hero shrew (Scutisorex somereni); and the cuttlebone from Sepia officinalis. These biological structures display contrasting functions and operate in different environments, and so present an interesting set of case studies to investigate using a common methodology.

There is a limited general understanding of the modifications of the hero shrew vertebrae and this study aims to non-destructively characterise the unique morphology of the vertebral column. The cuttlebone structure has previously been identified for applications in bonetissue scaffolds, and as a biomimetic material due to its complex microarchitecture. However the diversity of morphology in cuttlebone has not been documented previously, and therefore the aim is to characterise the complex morphologies in three-dimensions and link to the functional properties of the cuttlebone.

The structure-property relationships of these biomaterials are investigated through a range of multi-scale, multi-modal imaging techniques and the characterisation of their structures, chemical composition and material properties. Unique architectures are also reproduced using additive layer manufacturing to create scaled-up physical models.

The principal imaging method implemented across the two material systems is X-ray microtomography $(\mu \mathrm{CT})$, a non-destructive technique that allows visualisation of both internal and external structures of an opaque object. An X-ray $\mu \mathrm{CT}$ scanner acquires a series of projections from different angular positions, measuring the attenuation of the ionising radiation propagating through the sample. These projections are then used to reconstruct the CT data as a three-dimensional (3D) volume.

The materials investigated are mineralised structures in the form of calcium-carbonate and calcium-phosphate-based composites. The X-ray attenuation values of these mineralised structures work well with X-ray $\mu \mathrm{CT}$ to provide a $3 \mathrm{D}$ reconstruction that can be virtually manipulated. The ability to analyse the internal structures in 3D is important for viewing morphological variations across the structures, especially with respect to any change in function.

The study of biological materials and systems increases our knowledge of these from a research perspective. The application of advanced imaging, and in 3D, correlated with higher resolution electron microscopy characterisation provides a unique and holistic description of important biological architectures. Through characterisation of structures at varying length-scales, with emphasis on understanding the relationships with function, we can also use the concepts and principles as inspiration for the future development of bioinspired materials, alternative processing methods or applications in engineering and design. 


\section{Chapter 2}

\section{Review of Literature}

This chapter is divided into three principal sections and will give an overview of biological materials science, non-invasive imaging techniques used for the characterisation of biological materials and the application of X-ray microtomography in this field by reviewing relevant literature. Critical reviews of work pertinent to each case study are given at the beginning of each chapter.

\subsection{Biological materials science}

The first materials were natural and biological: stone, bones, wood, antler, shell, skins. Over time, these were succeeded by synthetic materials as humans learned to produce ceramics, glass and metals, which provided increased strength and improved performance of tools. With technological developments and advancements, modern materials have become increasingly complex and varied, offering superior performance in certain applications. However, there has been an increased revival of interest in natural and biological materials. This is due to their remarkable multifunctional properties, unusual architectures, superior mechanical performance, and the mild synthesis conditions in which they are achieved.

Interest in biological systems as structures is not a new idea, the first major work in this field was by D'Arcy W. Thompson [7], first published in 1917. He looked at biological materials as engineering structures and described relationships between form and mechanical function. Other works of importance are Currey's investigations into biomineralised biological materials [8] and Vincent's work on the mechanics of biological structures [1]. These investigations from a more mechanistic approach helped gain momentum in this emerging field.

Materials science is well documented as a multidisciplinary field at the interaction of physics, chemistry and biology. At the heart of this, is the relationship between structure and properties. This establishment of structure-property relationships is essential for the understanding of complex biological materials.

The field of biological-based materials science traditionally encompasses three principal 
but overlapping fields:

- Biological (or natural) materials: the materials and systems encountered in nature;

- Biomimetics or bioinspired materials: materials, devices and structures inspired from biological systems or functions;

- Biomaterials: synthetic materials specifically designed for biocompatibility.

Biological materials can be divided into two classes: structural and functional. Mechanical properties are considered the most important in structural materials, with strength, hardness, toughness and density being some of their principal characteristics. The primary attributes of functional materials can include attachment, optical properties and energy storage, and these functions are considered the important properties. As many biological materials are multifunctional, the boundaries between these classes can be unclear, however the primary attributes of the material can typically be divided into either the sustainment of the structure or a specific function.

\subsubsection{Fundamentals}

Biological materials are more complex than synthetic materials as they are often multifunctional and exhibit hierarchical structures. When looking at biological systems within this field, a variety of constraints and requirements demanded by nature must be considered; self-assembly, self-healing capability, evolutionary design and environmental constraints, hydration effects, mild-synthesis conditions, multifunctionality, and hierarchical design. These seven interrelated features have been identified by Artz [9] and further expanded by Meyers et al. and Chen et al. [10, 11, 12, 13]. In addition to these, a set of characteristic features specific to marine organisms has been proposed [14]. These are not shared by their terrestrial counterparts and include: complete hydration, variation in hydrostatic pressure, temperature, and salinity, as well as near constant motion from currents and swells. These constraints, whether terrestrial or marine, have shaped all structural biological materials.

An overview of the key differences between biological materials and synthetic ones is provided below.

Building blocks - biological materials are made up of a small number of constituents which are divided into two categories: biopolymers and biominerals. The most common biopolymers include collagen, keratin, chitin, cellulose and elastin [10, 11, 12, 15]. Common mineral building blocks are the calcium carbonates (e.g. calcite and aragonite), calcium phosphates (e.g. hydroxyapatite) and silica $[10,11,12,15,16]$. Many other examples of biopolymers and biominerals exist within natural materials with each providing a specific role. For example; being a structural component; a template for growth; or used for energy storage.

The materials formed from these constituents can be further identified into two groups from the mechanical property viewpoint [11]: 
1. 'Soft' biological materials - consist of biopolymers and generally have highly directed properties dependent on function.

2. 'Hard' biological materials - hierarchically structured composites of both biopolymers and biominerals, typically stronger in compression.

The 'hard' materials can be both mineralised and non-mineralised, as heavily crosslinked biopolymers can act as structural components, as seen in squid beaks (chitin) [17], horn (keratin) [18] and plant cell walls (cellulose) [19].

Self-assembly - in contrast to many synthetic materials, natural structures are assembled from the bottom-up, rather than from the top-down. This is necessary due to the lack of a pre-existing scaffold [12]. Complex processes of genetic messaging, and cellular and enzyme activity at the molecular level are fundamental to construct these self-assembled structures. Efforts are being made to try and replicate this design strategy in the manufacture synthetic structures as it has considerable technological potential [20, 21].

Self-healing capabilities - synthetic materials undergo damage and failure in an irreversible manner. In contrast, biological materials often possess the ability to reverse the effects of damage by healing. Cells embedded in the structure and vascularity are essential for the various repairing mechanisms used. The self-healing properties of bone have inspired a new class of composites [22].

Evolution and environmental constraints - the limited availability of elements (primarily $\mathrm{C}, \mathrm{N}, \mathrm{Ca}, \mathrm{H}, \mathrm{O}, \mathrm{Si}, \mathrm{P}$ ) determines morphology and the resultant properties of biological materials. There is an inherent conflict between different properties [23] such as stiffness and toughness, which means that biological structures may not be optimised for all conditions, however the evolutionary process leads to sufficient solutions. In addition, biological materials also have the ability to adapt to their environment, for example, bone undergoes modelling and remodelling in order to respond to external stimuli.

Hydration - many materials have properties that vary with the level of hydration. A notable example is a reduction in stiffness and strength but a higher toughness with increasing levels of hydration (for example, [24]).

Mild synthesis conditions - the majority of biological materials are formed at or ambient temperatures and pressures (approximately $300 \mathrm{~K}$ and $1 \mathrm{~atm}$ ). This is in stark contrast to many synthetic materials.

Multifunctionality - many components often serve more than one purpose within an organism. For example, bone provides structural support, whilst promoting growth of red blood cells and providing protection to organs.

Hierarchy - biological materials are organised by different length scales, from nanoto macro-scale and they have properties which translate between these scales. This organisation is inherent to their design, and the number of length scales is dependent on the complexity of the structure. The crab exoskeleton, for example, has six levels of hierarchy, 
from polysaccharide chitins at the molecular level to a bouligand structure at the micro-level $[25]$.

\subsubsection{Structural design elements}

Although there is a huge variety of organisms across a wide range of environments, there are clear repetitions in structures as organisms develop similar solutions to constraints and requirements imposed on them. As a result, eight principal structural designs have been identified by Naleway et al. [26] and are shown in Figure 2.1. The designs are all made up of biopolymers and biominerals and are assembled hierarchically.

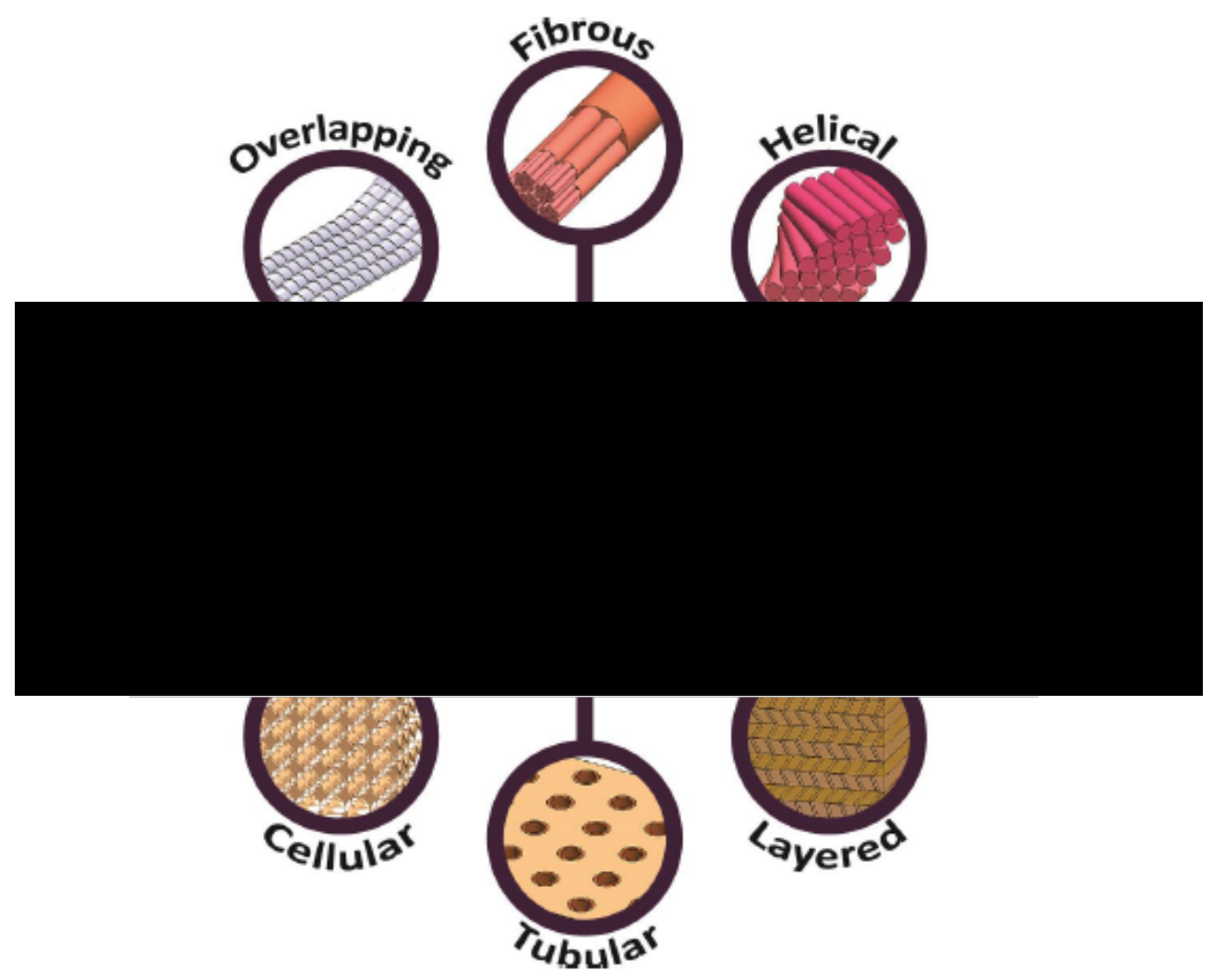

Figure 2.1: Eight principal biological structural design elements. Taken from [26].

Fibrous - typically found in biological materials that require high tensile strength or stiffness in a single direction. They are designed with numerous aligned fibres that often exhibit hierarchy across multiple length scales and generally occur within the nano- to microstructures of the materials. They are commonly found within non-mineralised, soft biological materials, such as silks [27, 28], but have also been observed in bone and arthropod exoskeletons where fibres of collagen and chitin respectively are mineralised [29, 30]. These structures initially allow a large amount of deformation with minimal energy consumption, followed by a large amount of energy consumption before fracture. 
Helical - common in composite or fibrous materials. They generally provide increased strength and toughness in multiple directions by stacking fibres or reinforcements at various angles. There are two main types of helical structures: twisted-ply structures that occur in non- or low-mineralised materials at a nano- to micro-scale and result in in-plane isotropy; and helically reinforcing structures that occur on exterior surfaces at a macro-scale and help to improve torsional rigidity. An idealised arrangement by Bouligand [31] describes how each layer is rotated by a constant angle until it completes a $180^{\circ}$ rotation. Many examples of helical structures found in biological materials exhibit increased toughness due to the increase of resistance to crack propagation from the differing orientations of layers, including the stomatopod dactyl club [4] and fish scales [32, 33, 34].

Gradient - occur in materials or interfaces where there is a property mismatch. To avoid stress build-up a gradual transition is employed, which can provide toughness, resist wear or prevent crack propagation. These structures vary in size from micro- to macroscale. Examples of gradient structures include those found in fish scales [35] and squid beak [17].

Layered - complex composites used to improve toughness of typically brittle materials through the introduction of interfaces. These interfaces have abrupt and often large changes in mechanical properties and occur in the microstructure. The brick-and-mortar structure of the abalone shell [36] is an excellent example of this layered structure.

Tubular - organised porosity that occurs at the microscale that can aid with energy absorption in compression and crack deflection. These structural elements are typically found in impact and pierce-resistant materials, including ram horns [37] and crab exoskeletons [25]. As well as improving fracture toughness and impact resistance, they also function to provide nutrients.

Cellular - lightweight porous or foam structures that provide directed stress distribution and energy absorption and therefore are capable of resisting bending and buckling. They are commonly seen in birds, where weight-savings are required, but are also seen in organisms to reduce weight in otherwise dense materials. These structures are seen either as the macrostructure as a bulk material, such as toucan beaks [6] or from the nano- to microscale within a composite, as in turtle shells [38]. When cellular structures are surrounded by dense layers they are known as sandwich structures.

Suture - interfaces of wavy or interdigitating patterns which control strength and flexibility. They generally consist of two phases; rigid suture teeth and a compliant interface. These sutures appear in the carapace of leatherback turtles [39], armadillo and alligator osteoderms [40, 41] and mammalian skulls [42]. Increased hierarchy of suture interfaces has been shown to significantly improve mechanical properties, and will generally have higher stiffness and toughness [43].

Overlapping - multiple plates, or scutes, are used to form a flexible protective surface at a macroscale. They are commonly used for armour and defence. The plates ensure constant coverage and protection of the organism whilst allowing flexibility. Numerous examples of overlapping structures include shark fins [44] and chiton exoskeletons [45]. 
The extraordinary mechanical properties observed in natural materials are typically a product of the intricate structural organisation at different spatial scales. By recognising the link between these structural design elements and their associated mechanical functions, insight can be made about the required function where they are found in biological materials.

\subsubsection{Biological materials}

Biological materials have been classified into four main groups by Wegst and Ashby [46]:

- ceramics and ceramic composites - where the mineral component is prevalent, including bone, shells and teeth;

- polymers and polymer composites - hooves of mammals, silk, ligaments and tendons;

- elastomers - materials that undergo large strains, such as skin, muscles and blood vessels;

- cellular materials - lightweight materials occurring in beaks, feathers, cancellous bone and wood.

Nature has evolved an expanse of biological materials to address different structural requirements such as, hardness, toughness, lightweight, stiffness. There is an inherent conflict between some properties, so a composite material is fashioned with an intricate ordering of structural components across length scales [47]. These composite materials combine the best properties from the constituents and have properties that far exceed those of the individual components [11, 23].

Ashby maps are a convenient way of concentrating a large amount of information into one diagram. Figure 2.2 shows classes of biological materials positioned in terms of strength and density. It is clear that the strength of biological materials is vast and varies over four orders of magnitude. Figure 2.3 shows the same classes of materials this time positioned by toughness and modulus. Calcite and mollusc shell have equivalent modulus values, however the mollusc shell is several orders of magnitude tougher, what is interesting is that mollusc shells are composed of more than $90 \%$ calcium carbonates. This emphasizes the importance of the hierarchical nature of structures in biological materials.

The hierarchical organisation in biological materials is inherent to their design. Hierarchical structures can be defined as a group of molecular units that are in contact with other phases, which are assembled at increasing length scales. Different materials exhibit hierarchy at several to many length scales depending on the complexity of the structure [48]. Figure 2.4 shows an example of this using bone and bamboo.

Many examples of bioinspiration and bioexploration can be found in the following literature along with in-depth examples of the different classes of biological materials $[10,11$, $12,46,50,51]$. 


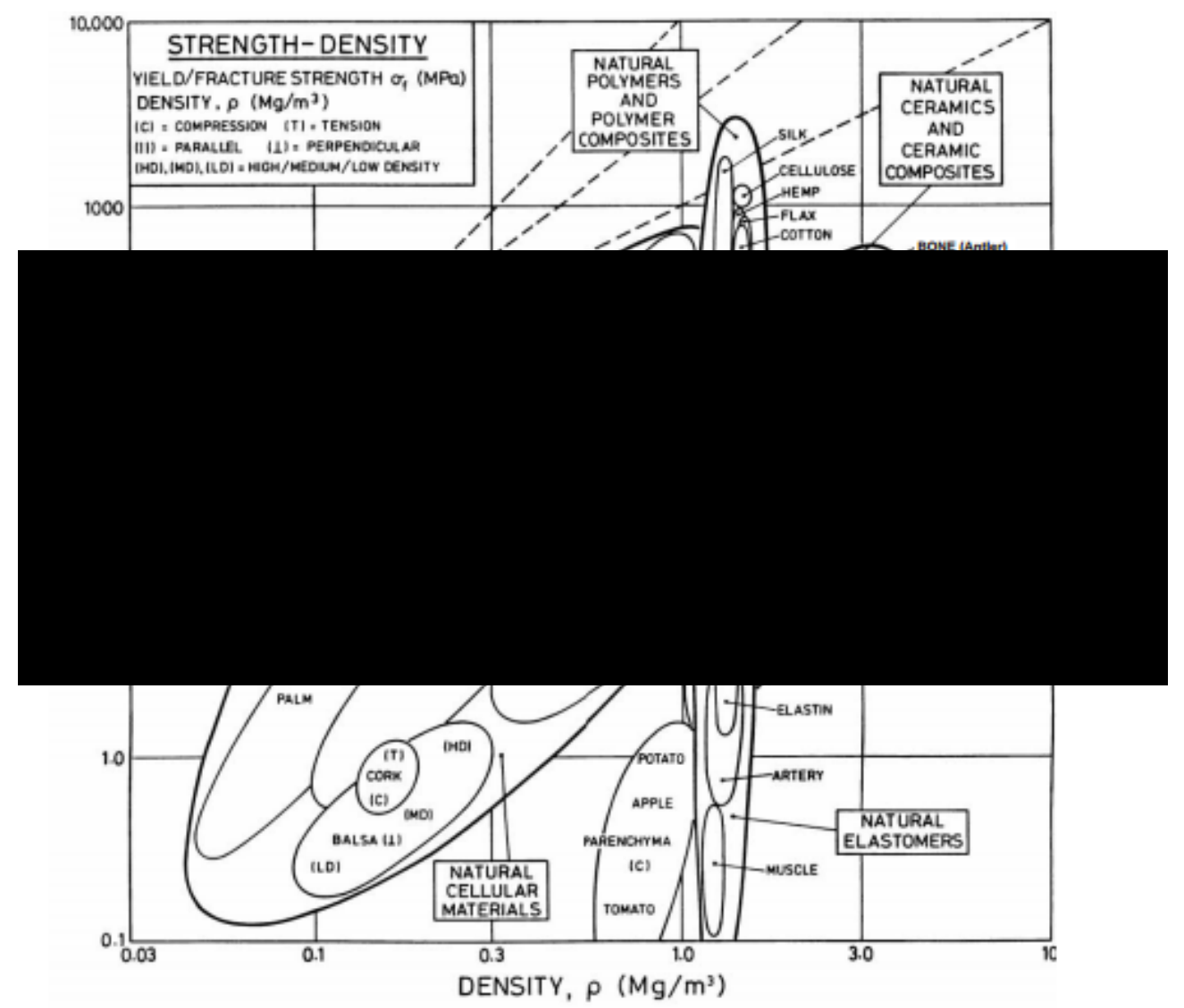

Figure 2.2: Ashby plot for biological materials showing strength as a function of density [46]. 


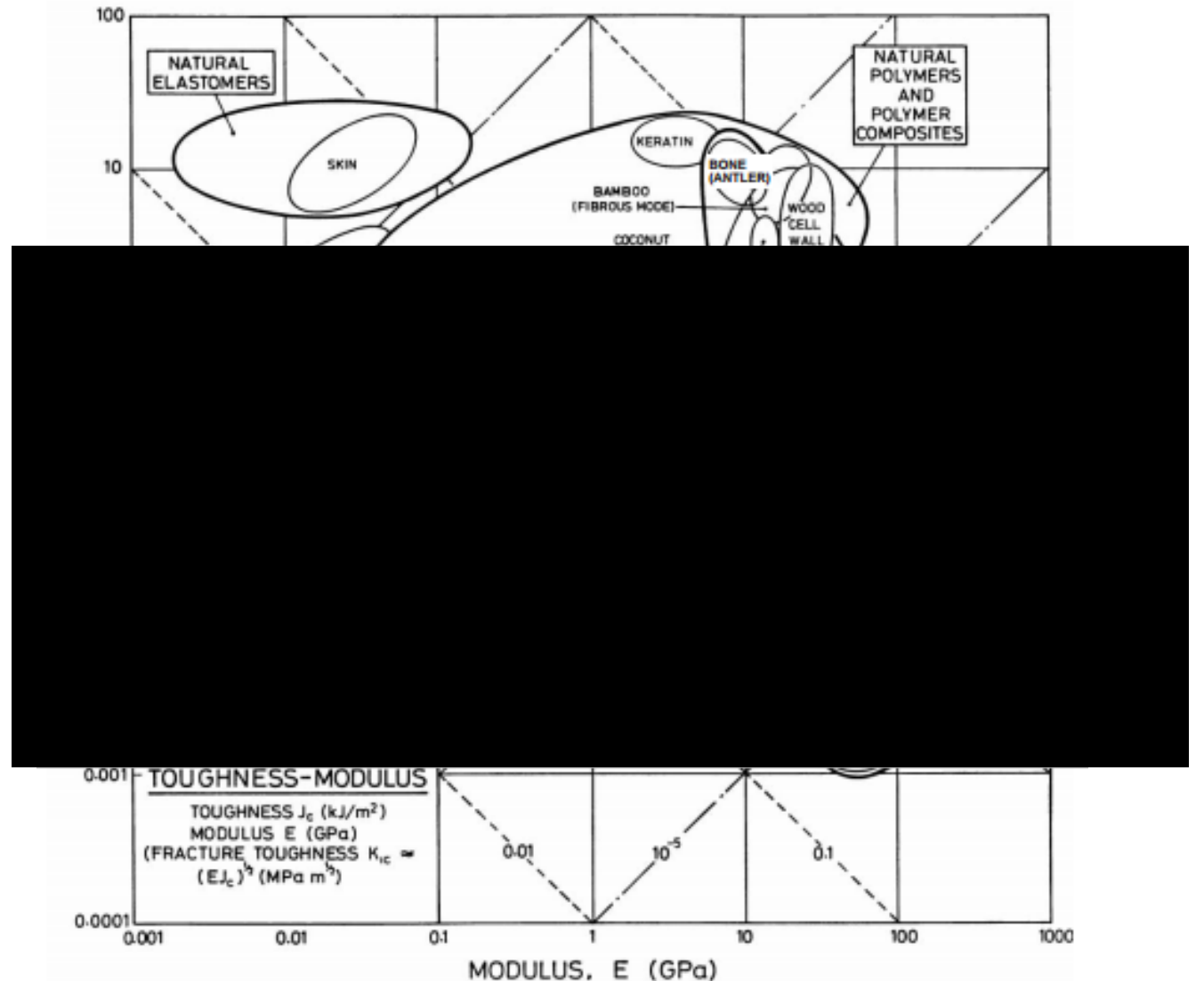

Figure 2.3: Ashby plot for biological materials showing toughness as a function of modulus $[46]$. 
a
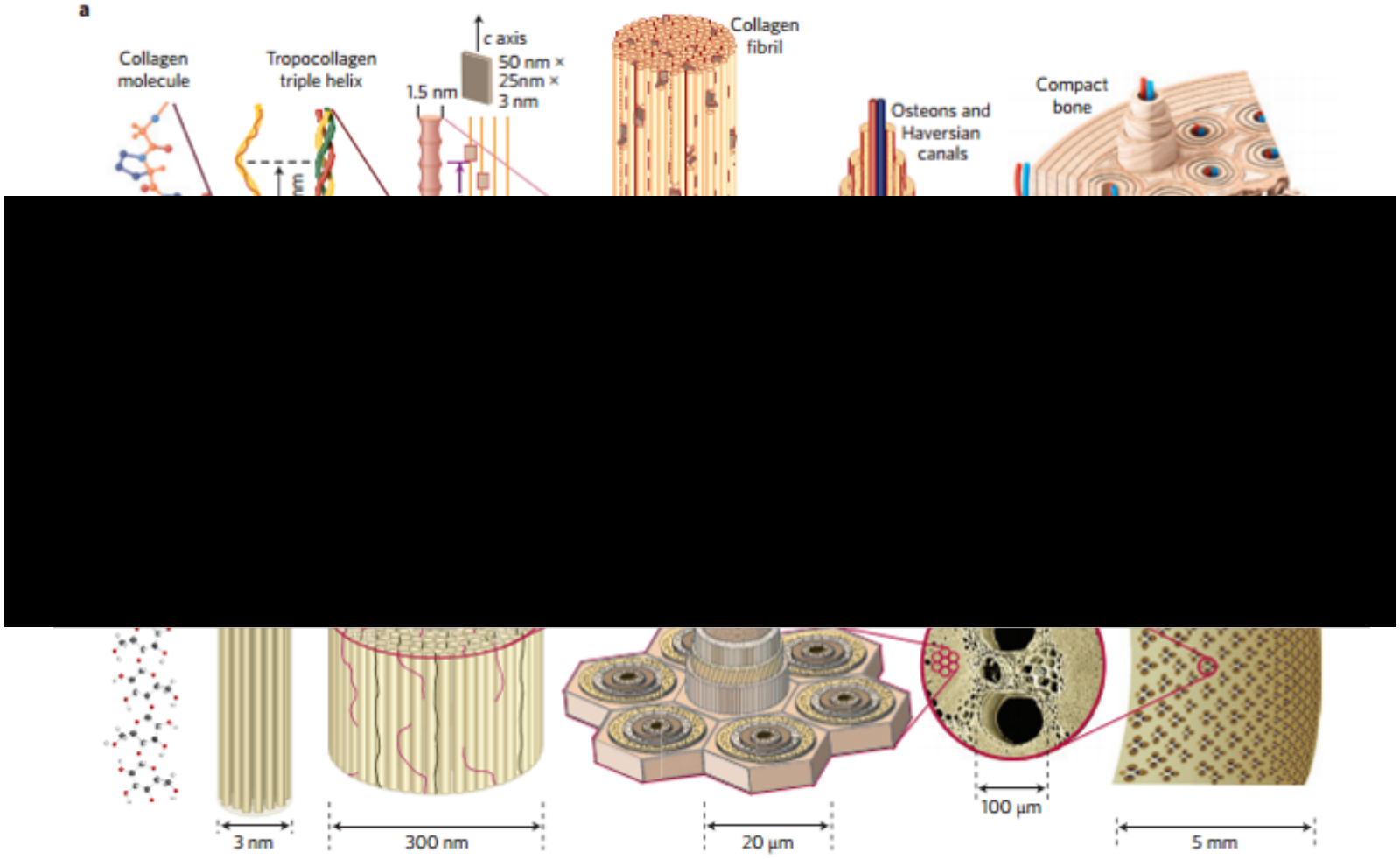

Figure 2.4: The hierarchical structure of (a) bone and (b) bamboo [49]. 


\subsubsection{Bioinspired materials and biomimetics}

Learning from biological systems, then applying modern engineering techniques in order to emulate these systems to solve complex engineering problems can be described as bioinspired design.

The origins of this field date back to the late 1950's and early 1960's. The term biomimetics was coined by Otto Schmitt and is defined in the Merriam-Webster dictionary as: "the study of the formation, structure, or function of biologically produced substances and materials (as enzymes or silk) and biological mechanisms and processes (as protein synthesis or photosynthesis) especially for the purpose of synthesizing similar products by artificial mechanisms which mimic natural ones" [52].

Many other terms have been proposed and used, however the general aim remains the same: to emulate the design and assembly principles used in natural materials. To achieve this, bioinspiration requires the identification, understanding, and quantification of natural design principles and their replication in synthetic materials [53]. This approach is being applied to both structural and functional materials and devices.

Although inspiration from nature is not a new concept (flying machines inspired by birds were first conceptualised by Da Vinci and later realised by the Wright brothers [54]), the rise of nanotechnology has made it possible to design and fabricate an array of advanced functional materials and devices inspired by nature [55].

There have typically been two approaches taken in the generation of bioinspired materials and structures [48]: traditional biomimetics and molecular-based biomimetics. Only the first of these approaches is considered here.

\subsubsection{The bioinspiration cycle}

The traditional bioinspiration approach is a linear path where one learns from nature to create a final design. The bioinspiration cycle proposed by Frank et al. [56] consists of four parts which learns from nature and provides benefit back to the understanding of biology, ecology and nature.

1. Biology: Develop an understanding of the materials and processes the organisms employ from a biological perspective. It is a collaborative process involving input from biologists, evolutionary biologists and ecologists. This is a necessary step to put the organisms into a biological context as misunderstandings can arise if researchers are ignorant e.g. of the anatomy and physiology, lifestyle (activities determine applied loadings). In addition, a clear understanding can help to identify organisms and behaviours that may be of interest in the development of new bioinspired designs.

2. Materials science: Use tools and techniques available in the fields of engineering, chemistry and physics to investigate organisms and understand how they function in their natural environments. Structures and behaviours can be analysed independently 
of their natural environment to determine their structural, mechanical, chemical and functional (e.g. optical or electromagnetic) properties.

3. Bioinspiration: Employ the lessons learned from nature to modern materials and techniques to form advanced designs that can bring benefit to society. Bioinspiration picks and chooses important structures, designs and concepts and employs them in novel designs.

4. Bioexploration: Develop a deeper understanding of biology and biological materials from the process of bioinspiration. This relatively recent aspect of the bioinspiration cycle was proposed by Porter [57].

\subsubsection{Investigation of biological design}

A framework for the investigation of biological design has been suggested by Frølich et al. [58]. The framework has been developed to aid in establishing relationships that identify the design parameters that are essential to materials' mechanical performance and are not simply artefacts of growth processes or evolutionary baggage. This combines parametric modelling based on structural characterisation, multi-material 3D printing, and direct mechanical testing. This framework allows for investigations of biological materials in a systematic and parametric way, rather than a typical correlation between computational models and their corresponding 3D prints. It creates an efficient and high-throughput experimental screening of a large design parameter space, therefore allowing the exploration of the structural design features that are vital in a materials' mechanical performance.

\subsubsection{Bioinspired materials}

Research in bioinspired design has led to the development of novel, high-performance engineering technologies that draw inspiration from natural biological systems. In addition to bioinspired materials, an assortment of bioinspired structures, devices and robotics [59, 60] have drawn inspiration from biological structures (e.g. buildings inspired by termite mounds [61]), mechanisms (e.g. light-harvesting devices inspired by photosynthesis [62]), and organisms (e.g. flying robots inspired by insects [63]).

One of the most famous examples of a commercialised biomimetic material is Velcro ${ }^{\circledR}$, which was invented by George de Mestral in 1941 after removing burdock burrs from his dog's fur [64]. Following observation of the burrs under a microscope de Mestral discovered the hook-and-loop mechanism - the inspiration for his invention.

A range of biologically inspired materials and designs have and are currently being investigated, for a broad set of applications, including, but limited to, fracture and impact resistance, armour and defence, cutting edges, aero and fluid dynamics, attachment, sensors and optical devices and medical applications. 
A few specific examples of structural and functional materials inspired by nature include: tough ceramics and composites inspired by abalone nacre [65, 66, 67]; cellular solids inspired by bone $[68,69,70,71]$; wet and dry adhesives inspired by mussel byssus and gecko toes $[72,73]$; resilient fibres inspired by spider silk $[74,75,76]$; self-cleaning surfaces and paints inspired by the lotus leaf $[77,78,79]$.

\section{$2.2 \quad$ Imaging techniques}

A thorough understanding of the interior of biological structures is essential for a better knowledge of their function. Destructive methods were traditionally the approach to visualising the internal structure by dissection or histological serial sectioning. Light microscopy or transmission electron microscopy were then used to visualise these sections in two dimensions. Scanning electron microscopes enabled the surfaces of these structures to be visualised as three dimensional objects due to its extensive depth of focus. Confocal laser scanning microscopy has produced 3D images of structures, such as insect morphology [80], however this can only be applied to transparent structures [81] and the sample size is limited.

The 3D internal structure of small samples could traditionally be reconstructed from 2D images of thin sections, however this is an elaborate and time-consuming process. Therefore a non-destructive approach is required for observing internal structure.

\subsubsection{X-ray Computed Tomography}

A key component in this study is the imaging technique of $\mathrm{X}$-ray micro-computed tomography $(\mu \mathrm{CT})$. To better understand the capabilities and limitations of the system, it is important to have a sound knowledge of the main concepts and principles behind the technology. A good understanding of what can be resolved and detected aids better interpretation and comprehension of the acquired CT data. The following provides background information on X-rays, computed tomography and how volume data is generated.

\subsubsection{X-ray physics and interaction with matter}

X-rays were discovered in 1895 by Wilhelm Conrad Röntgen. They are electromagnetic waves, with wavelength of approximately an Angström $\left(\AA, 10^{-10} \mathrm{~m}\right)$. Röntgen discovered that X-rays could penetrate through solid objects and be recorded on photographic film, creating a shadow image revealing the objects' internal structure.

$\mathrm{X}$-rays are generated when charged particles are accelerated or when electrons change shells within an atom, and can be produced in a laboratory-based setting (typically an X-ray tube, see Figure 2.5(a)), or at a synchrotron [82]. For a typical X-ray tube, electrons are emitted from a heated cathode filament (often tungsten) through thermionic emission and are accelerated towards a target anode by a voltage difference. The electrons collide with the material in the anode and X-rays are produced. 
There are two types of X-rays: (i) continuous X-rays, or bremsstrahlung and (ii) characteristic X-rays, as shown in Figure 2.5(b). Continuous X-rays/bremsstrahlung are produced when charged particles are decelerated due to collisions with the nuclei in the material. Characteristic X-rays are produced from electron transitions in the target material. Incoming electrons may collide with other electrons in the inner shells of the atom, causing the ejection of one of these. This creates a vacancy in the atom, which is filled by an electron from a higher energy level, emitting a photon with energy equal to the difference between the two energy states.

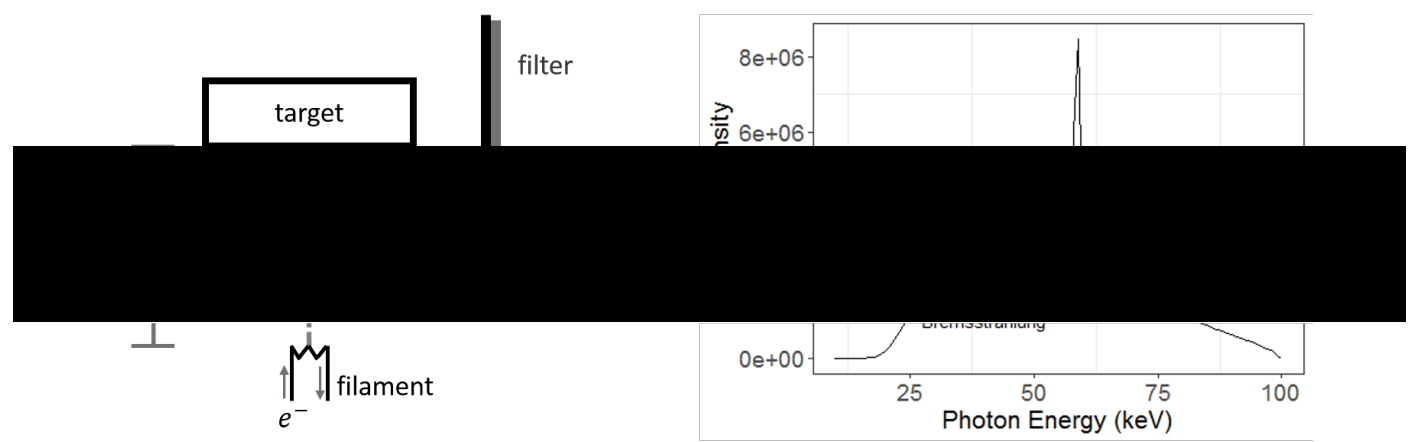

(a)

(b)

Figure 2.5: (a) Schematic of an X-ray tube (see text for description) [82]. (b) Plot showing the difference in photon energy distribution of bremsstrahlung and characteristic X-rays. Plot generated using SpekCalc [83] software for a tungsten target and acceleration voltage of $100 \mathrm{kV}$.

For energies up to $1 \mathrm{MeV}$, there are three primary processes in which X-rays interact with matter: the photoelectric effect, Compton scattering and Rayleigh scattering. The first two of these are the processes relevant to the X-ray energies used in CT systems. The photoelectric effect occurs when a photon's energy is transferred to an electron, which is then expelled from its orbit. This generally increases with atomic number $Z$ of the material, therefore materials such as bone (calcium and phosphorus) are more absorbing than soft tissue. Compton scattering occurs when a photon collides with an outer shell electron resulting in a photon of lower energy with a changed direction, and a recoil electron. These processes all contribute to the linear attenuation coefficient $\mu$ which is a measure of the probability of an X-ray interacting in a given material. It is dependent on the atomic number $Z$ and density $\rho$ of the absorbing material and varies with X-ray energy $E$.

As an incident X-ray beam passes through a material of density $\rho$ and thickness $x$, the beam will be attenuated due to absorption and scattering mechanisms. This attenuation follows the Beer-Lambert law:

$$
I(x)=I_{0} e^{-\mu x}
$$

where $I(x)$ is the intensity after it traverses a thickness of material $x$ with a linear attenuation coefficient $\mu$ and $I_{0}$ is the intensity of the unattenuated X-ray beam. $\mu$ can be normalised 
by the density of the material and is termed the mass attenuation coefficient $\mu / \rho$, in units of $\mathrm{cm}^{2} / \mathrm{g}$.

For a composite material with different attenuation characteristics, if the intensity of the incident photons $I_{0}$ and the intensity of photons after exiting the material $I$ are known, the total attenuation of photons across the material is equal to a line integral

$$
\int_{\text {ray }} \mu(x, y) d s=\ln \frac{I_{0}}{I}
$$

where $\mu(x, y)$ is the attenuation coefficient as a function of its spatial coordinates.

The attenuation coefficient is also a function of the energy of the incident X-ray beam, therefore the Beer-Lambert law is only valid for monochromatic X-rays. It is worth mentioning that the mathematics of computed tomography reconstruction are derived based on the assumption that X-ray attenuation follows the Beer-Lambert law. However due to the polychromatic nature of X-rays produced in lab-based CT systems, artefacts may be present in the reconstructed data. For a monochromatic beam, as seen in (2.2.1.2), the experimental measurement of $\ln \left(I_{0} / I\right)$ is linearly proportional to the absorber thickness.

\subsubsection{Computed Tomography}

X-ray computed tomography $(\mathrm{CT})$ is a technique that allows for the non-destructive acquisition of both interior and exterior structures of an object with X-rays. The process of X-ray $\mathrm{CT}$ is summarised in Figure 2.6. An X-ray source emits a beam (in our case, a cone-beam) of X-rays that are attenuated as they propagate through an object. A detector measures the energies of the X-rays that fully penetrate the sample, the output of which is a transmission image, termed projection or radiograph. Projections are taken from multiple angular positions, usually through $360^{\circ}$. These projections are reconstructed to create tomograms, which describe the material distribution on any plane. These tomograms can be stacked to produce a CT volume which can be visualised and manipulated digitally.

The first CT scanner was pioneered by Godfrey Hounsfield as a medical diagnostic tool in the 1970s [84]. Due to the benefits gained from being able to visualise an object's internal structure non-destructively, X-ray CT has been applied to many fields outside medicine. These include palaeontology [85], aerospace engineering [86], geology [87] and evolutionary biology [88].

Reconstruction Reconstruction is the process of estimating an object's cross-section from its projections. There are generally two approaches to solving the inverse problem of reconstruction: (i) using an analytical approach by formulating a solution in a closed-form equation, and (ii) attempting to solve it as an optimisation problem using an iterative approach [89]. Both methods have their own advantages and disadvantages, with analytical approaches being more computationally efficient, whilst iterative methods can improve 


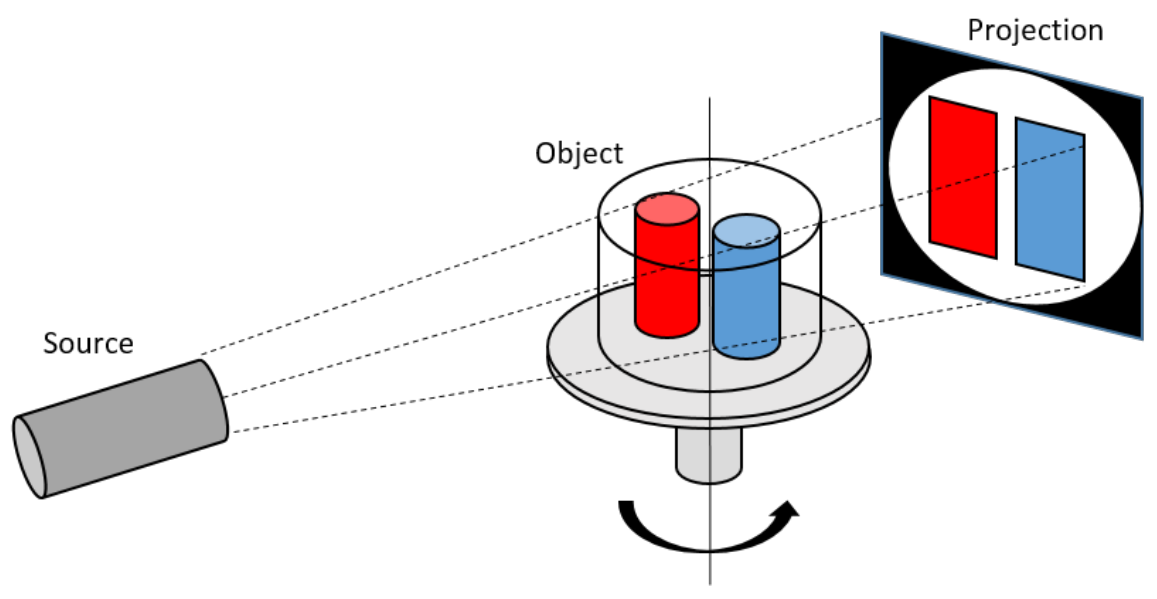

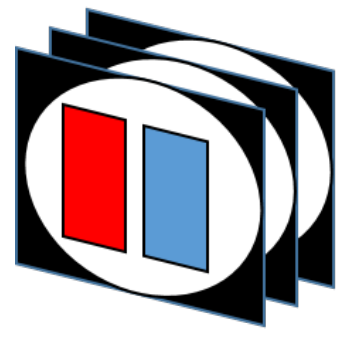

Projections for multiple angular positions

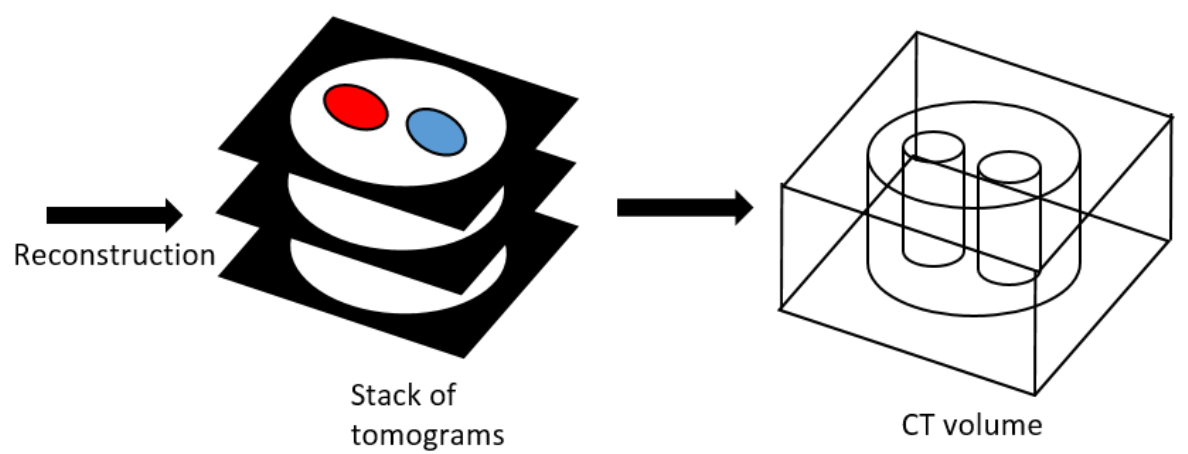

Figure 2.6: Sketch of projection acquisition for a cone-beam CT system, with the resulting process of reconstruction leading to visualisation of tomograms and a CT volume. 
image quality [90]. Only analytical reconstruction is applied in this thesis, therefore a brief description is provided.

Analytic reconstruction methods are based on the Fourier slice theorem, where the mathematics can be seen in other texts [91, 92]. In principle, the cross-section of the object to be reconstructed is considered as a $2 \mathrm{D}$ function $f(x, y)$, whilst the X-ray attenuation measured at each detector pixel is considered a line integral of the object function, denoted $P(\theta, t)$, where $\theta$ is the projection angle and $t$ is the detector column. The 1D Fourier transform of a projection $S(\omega)$ is equivalent to a radial line of the 2D Fourier transform of the original object $F(u, v)$. Therefore, by acquiring projections at multiple angular positions, the $2 \mathrm{D}$ Fourier transform can be filled and transformed inversely to give the original object function. This is demonstrated in Figure 2.7.

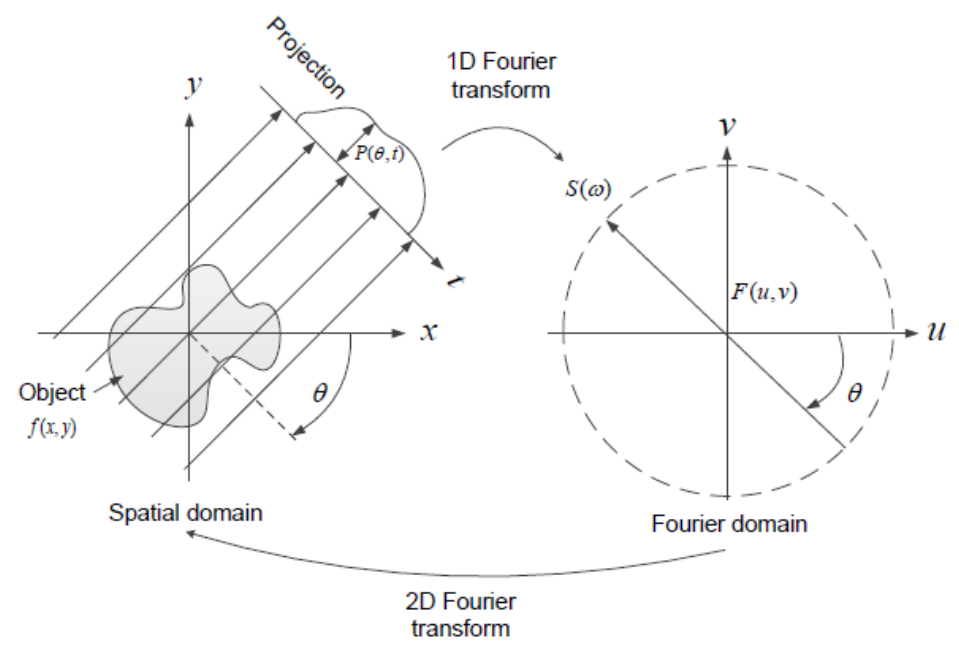

Figure 2.7: Illustration of the Fourier slice theorem [93].

The term $P(\theta, t)$ is the Radon transform of the function $f(x, y)$ and applying this at different angles to the object $f(x, y)$, a series of line projections are obtained, known as a sinogram (Figure 2.8). The algorithm used most commonly in CT data reconstruction is the filtered back-projection algorithm. Each line from the sinogram is superimposed over a square grid at the angle that corresponds to its acquisition and the final pixel value is determined by the summation of these back-projections. This is illustrated in Figure 2.8.

Artefacts Artefacts are the artificial features that appear in CT data but do not correspond to the physical features of the object. These artefacts reduce the quality of CT data and can arise from a range of sources, including the scanned object and reconstruction algorithm. Beam hardening and ring artefacts are discussed here but more information about the various types of artefacts can be found in [93, 95].

A tomogram's grey levels are related to their attenuation, and therefore the material properties. The grey levels in a tomogram can be affected by these artefacts, therefore it is 


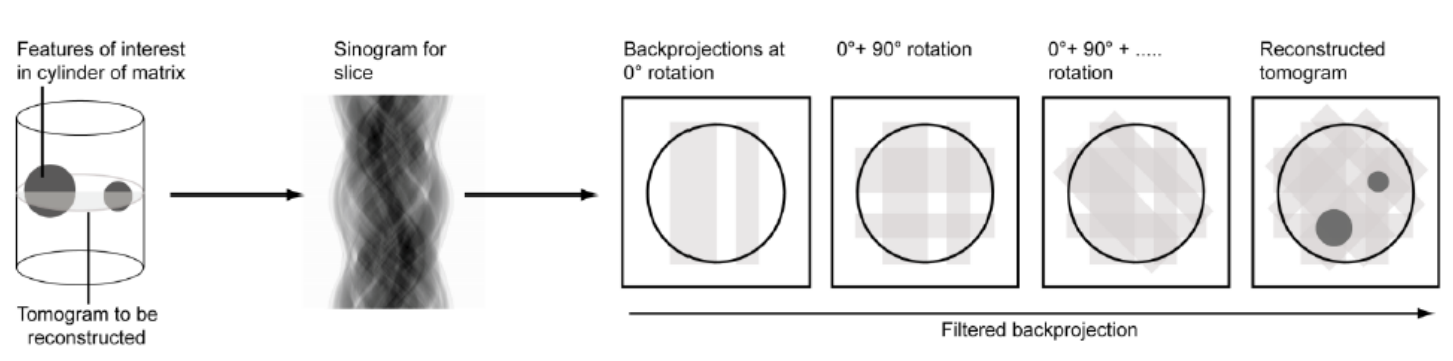

Figure 2.8: Illustration of the reconstruction process of a sample, resulting sinogram and process of filtered back-projection [94].

essential to understand them and how to minimise their effects. Figure 2.9 shows examples of some common artefacts seen in X-ray $\mu \mathrm{CT}$.

Beam hardening (Figure 2.9(a)) is one of the most common artefacts encountered and is caused by the uneven attenuation of X-rays from a polychromatic source [96]. Low energy photons are preferentially absorbed causing the mean energy of the X-ray spectrum to increase. The more material an X-ray beam penetrates, the more low energy electrons are removed from the spectrum making it increasingly difficult to attenuate the beam further. This leads to a characteristic dishing effect where the specimen appears less dense in the centre [95]. For irregular objects it may be difficult to distinguish between beam hardening effects and actual material variations. Ring artefacts result from shifts in output of individual detectors or sets of detectors [97]. This causes the superposition of concentric circles centred on the rotation axis of the tomogram, as seen in Figure 2.9(b). To overcome this, slight movement of the detector or sample between projections can break up the reinforcement of the artefacts and reduce their severity [95]. The impacts of both beam hardening and ring artefacts can be minimised using a combination of hardware (use of a beam filter to remove lower energy X-rays, improved detector calibration) and software solutions (wedge corrections) [85, 95, 97].

\subsection{X-ray microtomography in biological materials science}

The ability to visualise structures in three dimensions through non-destructive analysis has made X-ray $\mu \mathrm{CT}$ an attractive method for considering biological materials. X-ray $\mu \mathrm{CT}$ allows for internal and external features to be analysed and any morphological variation to be captured in 3D. It is also an extremely useful tool for correlative studies and hierarchical structure investigations.

An extremely broad amount of research has been conducted in this field. Key features of $\mathrm{X}$-ray $\mu \mathrm{CT}$ in biological materials science, such as sample preparation and advanced techniques for imaging of soft tissue are given and areas of research considered relevant to this thesis are reviewed. 


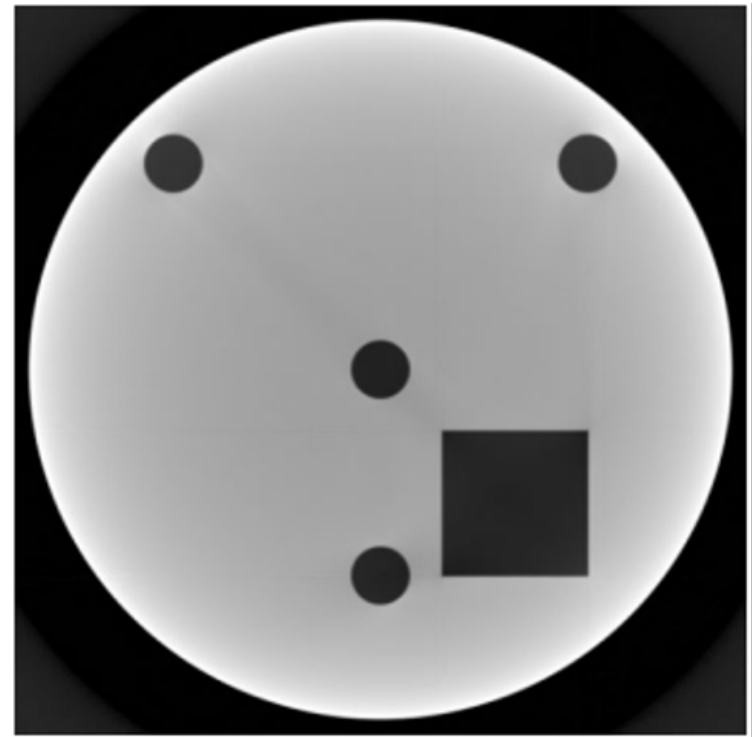

(a)

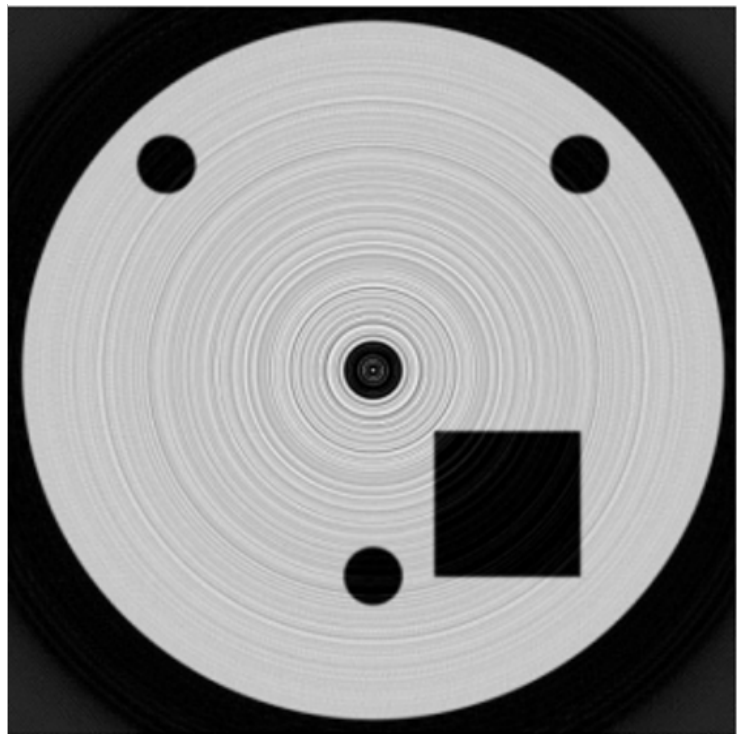

(b)

Figure 2.9: Examples of artefacts in X-ray $\mu \mathrm{CT}$ [95]; (a) beam hardening, (b) ring artefacts.

\subsubsection{Sample preparation}

Sample preparation for biological materials is dependent on the characteristics of the material in question. Understanding the material composition is critical to producing successful results. Until the arrival of phase contrast imaging, absorption was the only contrast mechanism that could be used for imaging, so only certain materials with high X-ray attenuation coefficients could be analysed [81].

Organic materials - these materials are compounds of low-atomic number. They are almost transparent to conventional X-rays and so require some form of contrast enhancement, such as staining or phase contrast imaging, in order to be visualised.

Inorganic materials - typically do not require any specialist sample preparation as conventional absorption contrast imaging is well suited as these materials have a high X-ray attenuation coefficient

\subsubsection{Staining}

The staining agents used in X-ray $\mu \mathrm{CT}$ are very similar to those used for staining histological samples in optical, fluorescence and electron microscopy [81, 98]. These agents typically have a much higher mass attenuation coefficient due to the presence of elements with a high atomic number. Figure 2.10 displays a comparison of volumetric data acquired by $\mathrm{X}$-ray $\mu \mathrm{CT}$ using no contrast enhancement (top row) and a sample stained with phosphormolybdenic acid (bottom row). Staining protocols can be found in the literature. For example; plant tissues [99], embryonic tissues [100, 101], and animal soft tissues [98]. 


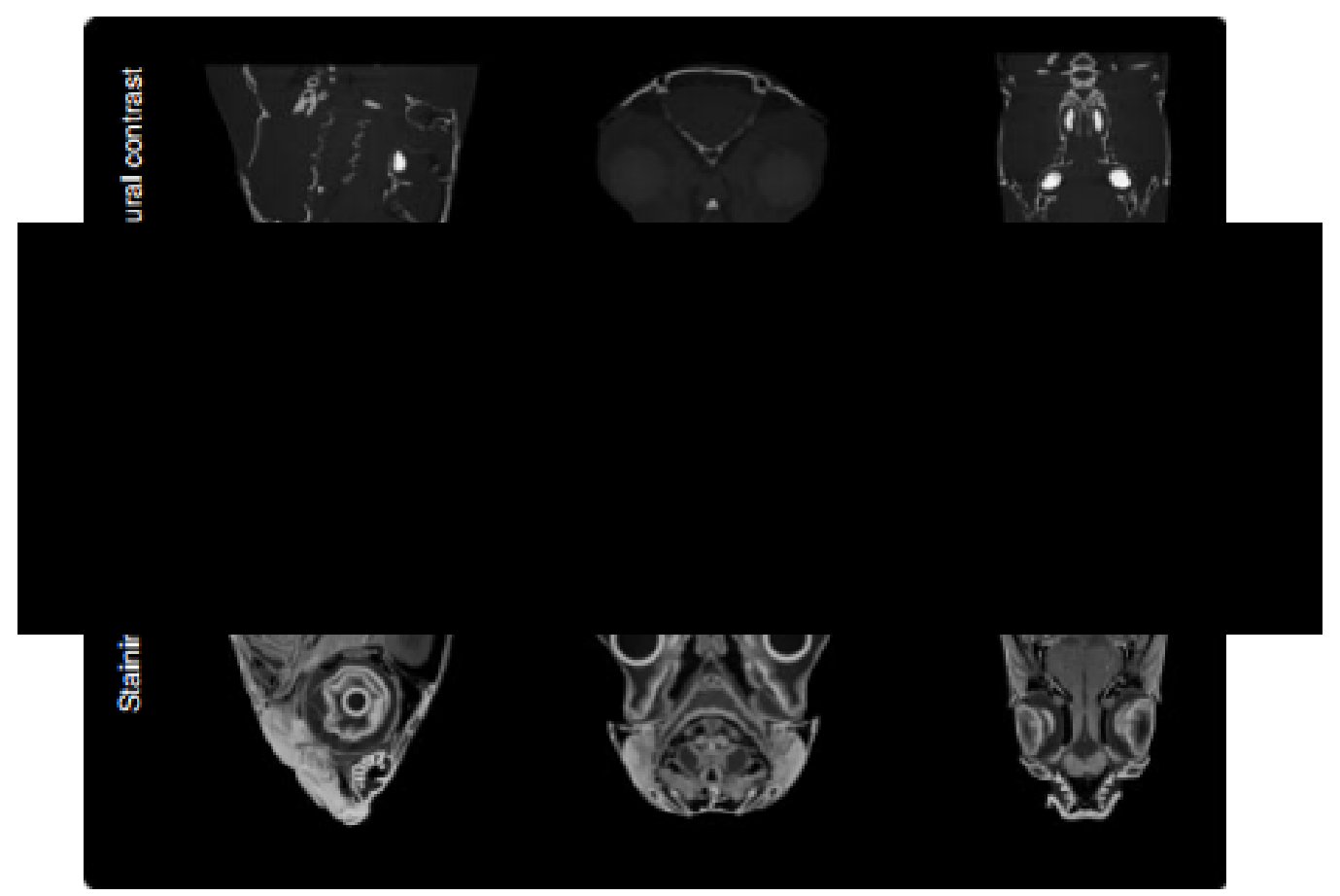

Figure 2.10: Effects of staining in absorption contrast X-ray $\mu \mathrm{CT}$ of a golden ide (Leuciscus idus) [102]. The top row of images are the sample with no staining, the bottom row is sample stained with phosphor-molybdenic acid.

\subsubsection{Phase contrast imaging}

Phase imaging [103] enables the mapping of changes in the refractive index when an Xray beam penetrates a sample. It is useful as a way of increasing the contrast between objects that attenuate the X-ray beam in a similar manner, such as non-mineralised biological materials. Enhanced edge contrast between phases can make follow-on processes such as segmentation easier or possible [90]. Figure 2.11 shows a comparison between the process of conventional absorption contrast tomography (top) and phase contrast tomography (bottom). Phase imaging methods include free-space propagation [104], grating interferometry [105], Zernike phase contrast [106] and analyzer-based methods [107].

\subsubsection{Bioinvestigation from functional designs}

$\mathrm{X}$-ray $\mu \mathrm{CT}$ has been applied across a multitude of different biological materials. Examples of $\mu \mathrm{CT}$ investigations, along with complementary analysis methods, are given below.

\subsubsection{Fracture and impact resistance}

Fracture and impact resistance is a function of many biological materials for a variety of reasons. Some species use impact to break other materials for food or shelter, whilst others require fracture and impact resistance to survive fighting and combat. 


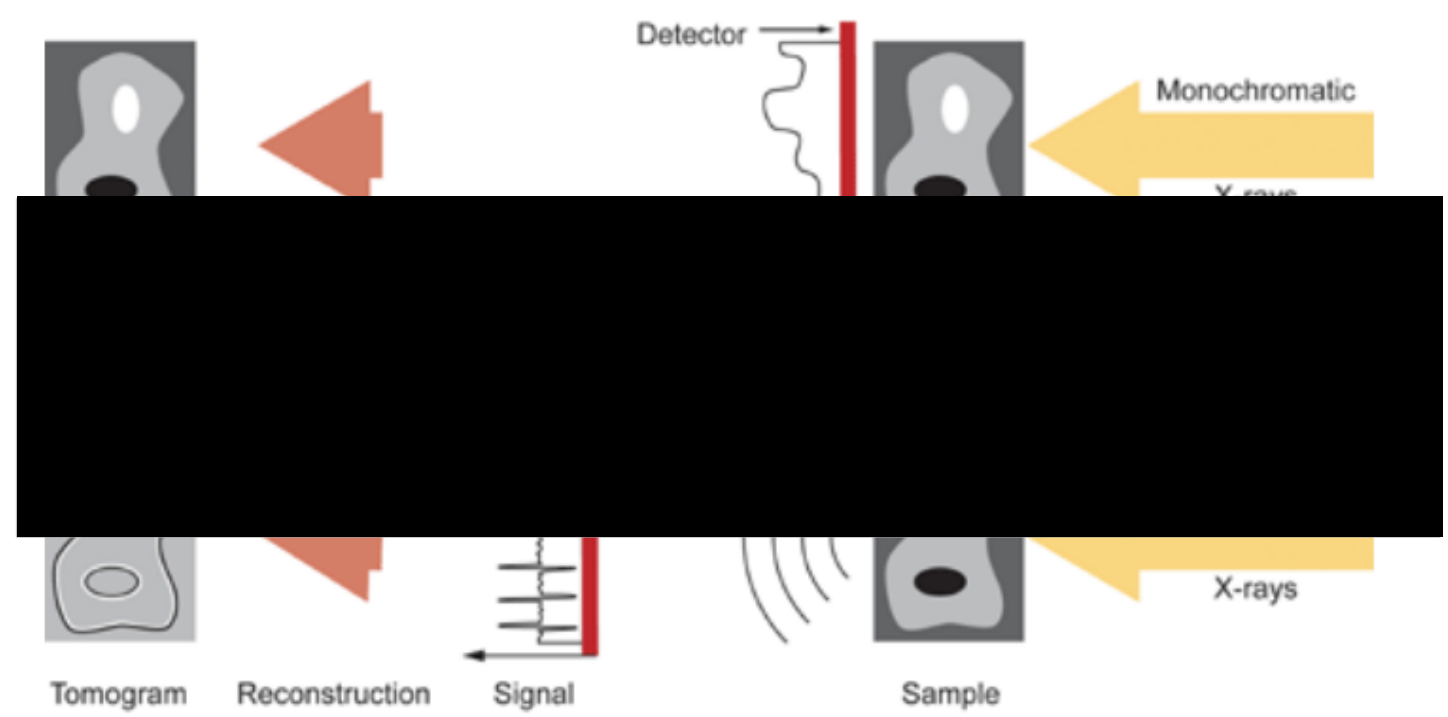

Phase Contrast: Large sample-detector distance

Figure 2.11: Conventional absorption contrast tomography (top) and phase contrast tomography (bottom) [81].

Jung et al. [108] investigated woodpeckers and their ability to avoid brain injury when pecking at trees, with a multi-scale structural analysis and mechanical property evaluation. A 3D model was developed using $\mu \mathrm{CT}$ image analysis and used for quantitative analysis of cross-sectional changes of the hyoid bone. They also applied SEM and EDS to reveal the microstructure and chemistry, and nanoindentation to show that the hyoid bone crosssections consist of a dense interior region with a porous compliant surrounding region, which may be effective at dissipating energy during pecking. These findings may have implications for the design of impact-absorbing structures.

Taylor et al. [109] used $\mu \mathrm{CT}$ to determine mineralisation patterns of the telson of a mantis shrimp (Stomatopoda). The telson acts as a 'punch bag' to resist the extreme impacts of the raptorial appendages of other mantis shrimp. They showed high levels of mineralisation in the impact area, which when coupled with the unique design of the telson, helps to dissipate the impact. The telson is also a great example of bioinspiration for armour.

Launey et al. [110] used 3D $\mu \mathrm{CT}$ visualisations to analyse crack paths and the distributions of micro-damage after stress testing in elk antlers, which in the traverse orientation is one of the toughest known biological materials.

\subsubsection{Armour and defence}

The ability to exhibit an effective defence through the use of armour, camouflage and other methods is an essential function for many species. They have been analysed at great length to inspire designs for a wide range of applications, including protective clothing and military 


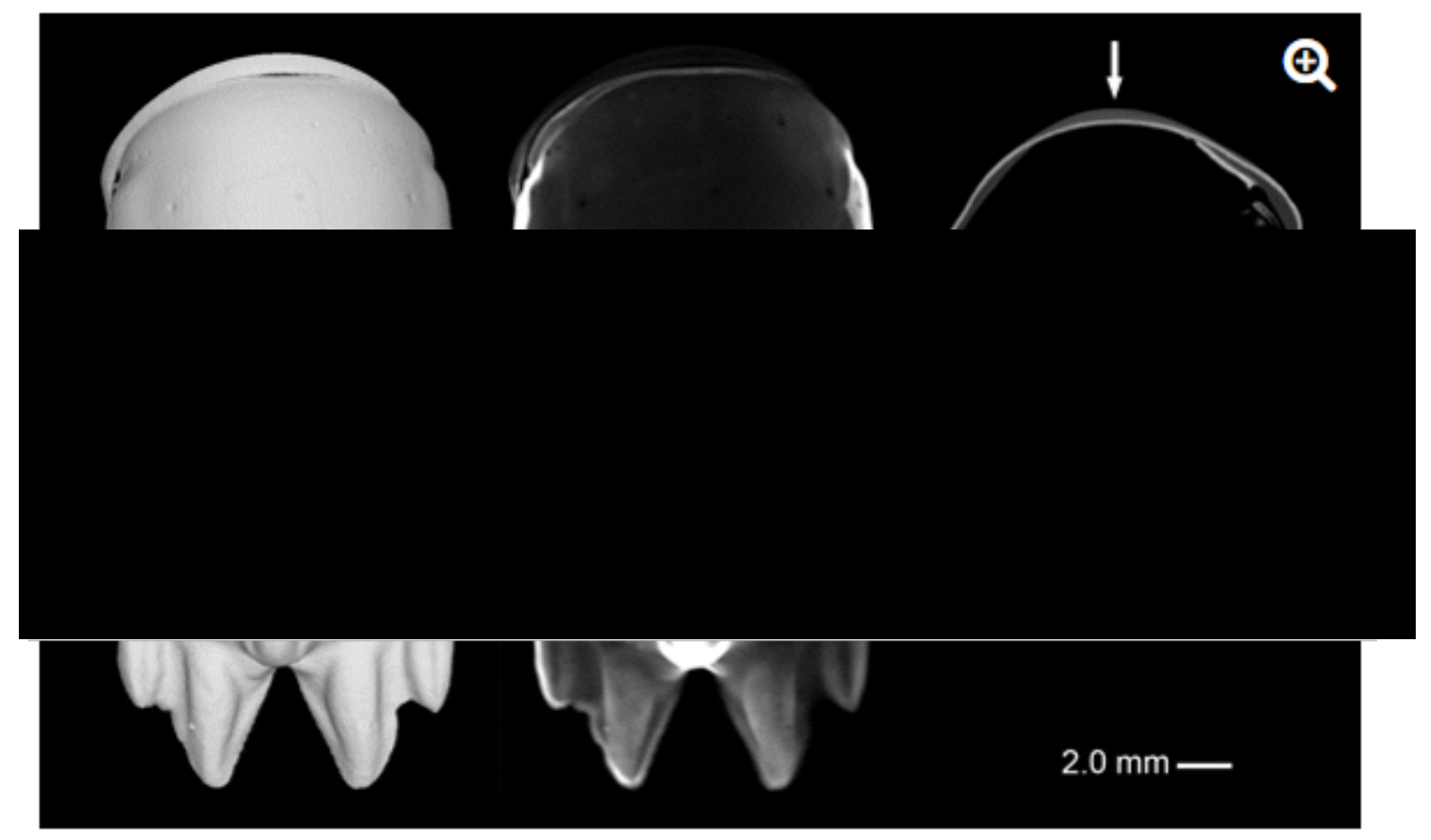

Figure 2.12: $\mu \mathrm{CT}$ images of fifth abdominal tergite (top) and the telson (bottom) [109]. Left images show surface conformation, centre images show relative mineralisation with bright white indicating greater mineralisation, right images show traverse sections of cuticle thickness at midpoint of the structure. 
equipment.

Yang et al. [111] investigated the armoured carapace of the boxfish, a feature linked to protection at the cost of mobility and speed. $\mu \mathrm{CT}$ was used to consider the micro- and macroscale 3D architecture of the boxfish and individual scutes. These scutes, shown in Figure 2.13, are connected together by interlocking suture interfaces which resemble triangular teeth. SEM was also used to propose that the sutured interface either accommodates for changing pressures of the environment or is linked to growth.

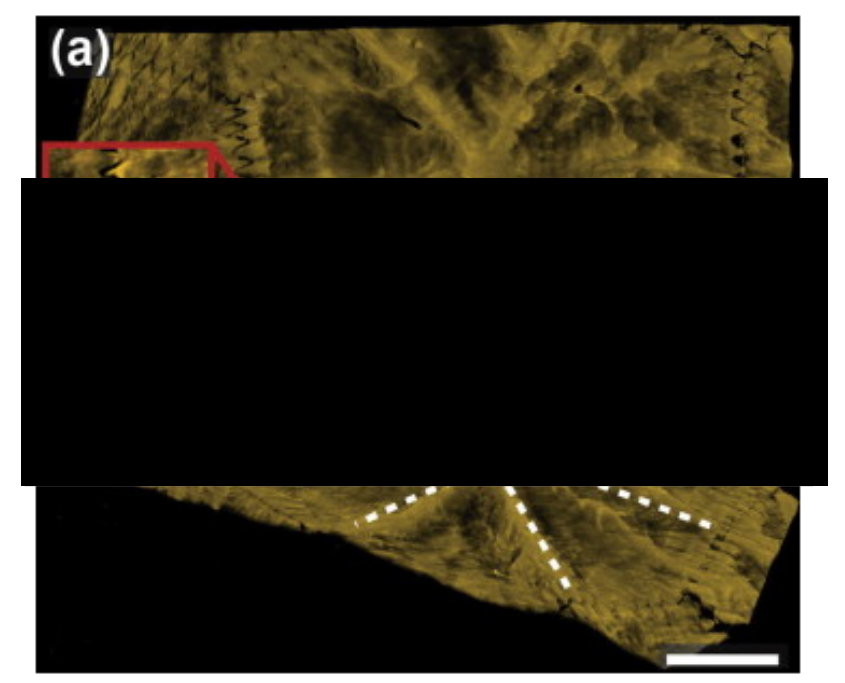

Figure 2.13: High-resolution $\mu \mathrm{CT}$ images of a boxfish scute [111]. (a) Top view showing interlocking hexagonal scutes, (b) profile view showing six raised struts extending from the centre to the edges of the scute.

Sun et al. [112] used a complementary set of analyses to look at osteoderms of the alligator, which provide both protection and flexibility. They used $\mu \mathrm{CT}$ to identify a complex neurovascular network. SEM then showed that osteoderms consist of woven bone in the dorsal region and lamellar-zone bone in the ventral. Finally, nanoindentation and compressive tests were performed to understand the mechanical properties which showed a gradient structure from the stiff dorsal cortex to the compliant ventral base.

Porter et al. [113] investigated deformations of seahorse plates which surround the vertebrae using $\mu \mathrm{CT}$. During compression, the interlapping plates slide past each other, allowing the tail to endure remarkable amounts of compression before any failure. Even during failure, the plastic response of local buckling of the tail means that the central vertebrae is protected from fracture. Figures 2.14 and 2.15 show various views of the tail, and the tail's responses to compression, respectively. Possible future biomimetic devices were suggested as steerable catheters, flexible armour and earthquake resistant structures. Porter et al. [114] continued this work by using 3D printing to help understand why seahorses have a square tail, in contrast with the cylindrical tail of most animals. Prototypes for a square and cylindrical tail were built and compared. The square tail was shown to be superior for 
two notable functions: grasping ability and crushing resistance.

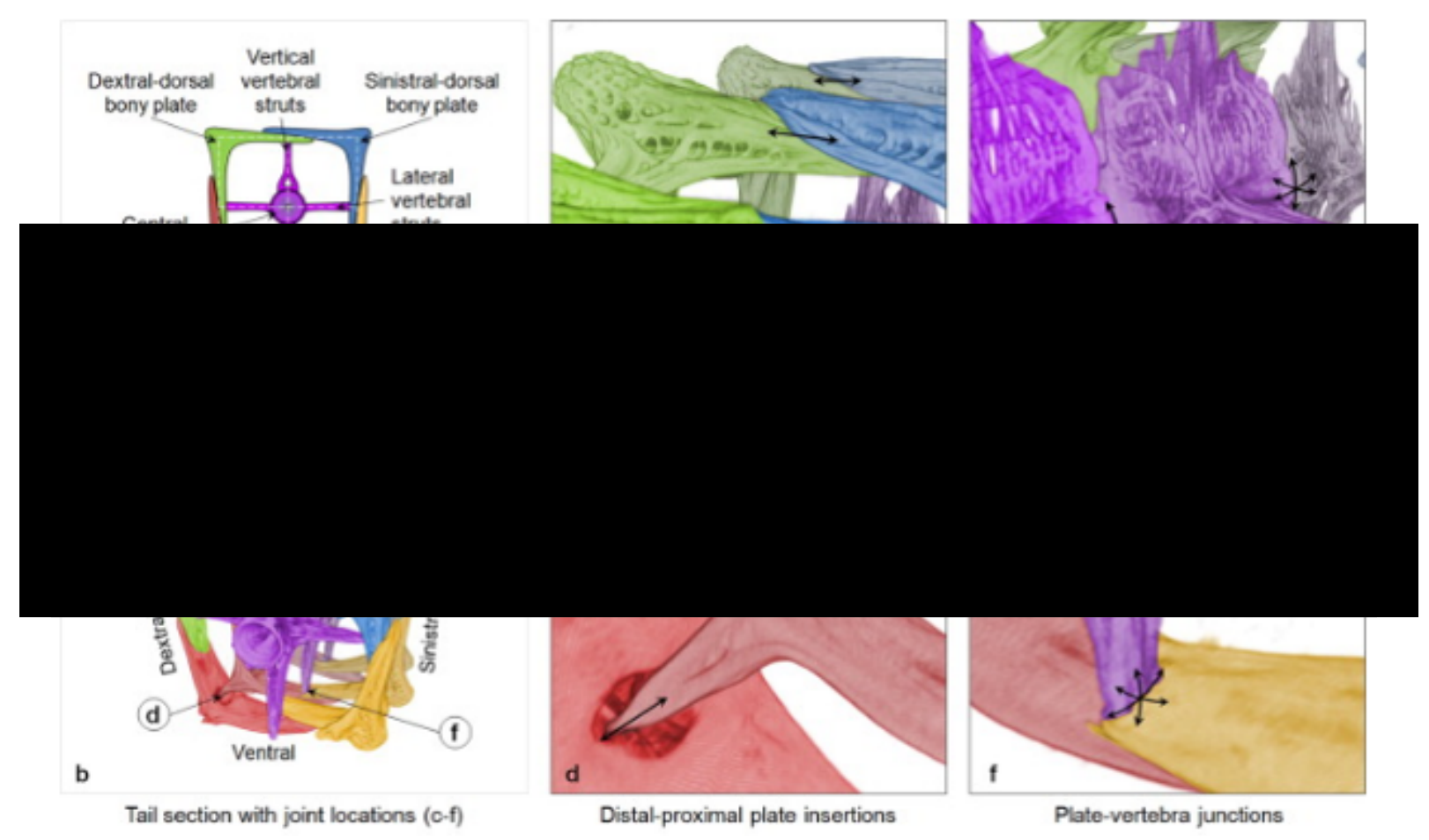

Figure 2.14: Seahorse tail cross-sections and $\mu \mathrm{CT}$ images [113]. (a) Schematic of tail (distal view) denoting each bony plate and vertebrae, (b) $\mu \mathrm{CT}$ image of tail indicating the four main joints, (c) lateral plate overlaps and gliding joints, (d) distal-proximal plate insertions and gliding joints, (e) vertebrae connections and pivoting joints, (f) plate-vertebrae junctions and pivoting joints. Arrows indicate directions of gliding (c and d) and pivoting (e and f).

\subsubsection{Aero and fluid dynamics}

Aerodynamic activity requires stiff, lightweight structures to enable efficient movement. Therefore these structures need to be optimised for weight whilst providing enough stiffness for structural support. Marine organisms are designed to move through fluid as efficiently as possible by reducing drag to minimise energy expenditure.

Seki et al. [115] use $\mu \mathrm{CT}$ to examine the internal and external structures of toucan and hornbill beaks. Beaks need to be strong whilst lightweight to not impact flight. They found that both beaks have a keratinous exterior and a bony foam interior. The foam interior consists of a cellular structure with trabeculae and thin membranes and increases the beak's resistance to bending compared to hollow cylinder with the same mass.

Wen et al. [116] investigated the form and function of shark skin using $\mu \mathrm{CT}$ imaging to create 3D printed denticles, shown in Figure2.16. A section of skin was created for use as a test rig. The results showed some evidence of increase swim speed and reduced swimming energy. 


\subsection{X-RAY MICROTOMOGRAPHY IN BIOLOGICAL MATERIALS}

SCIENCE

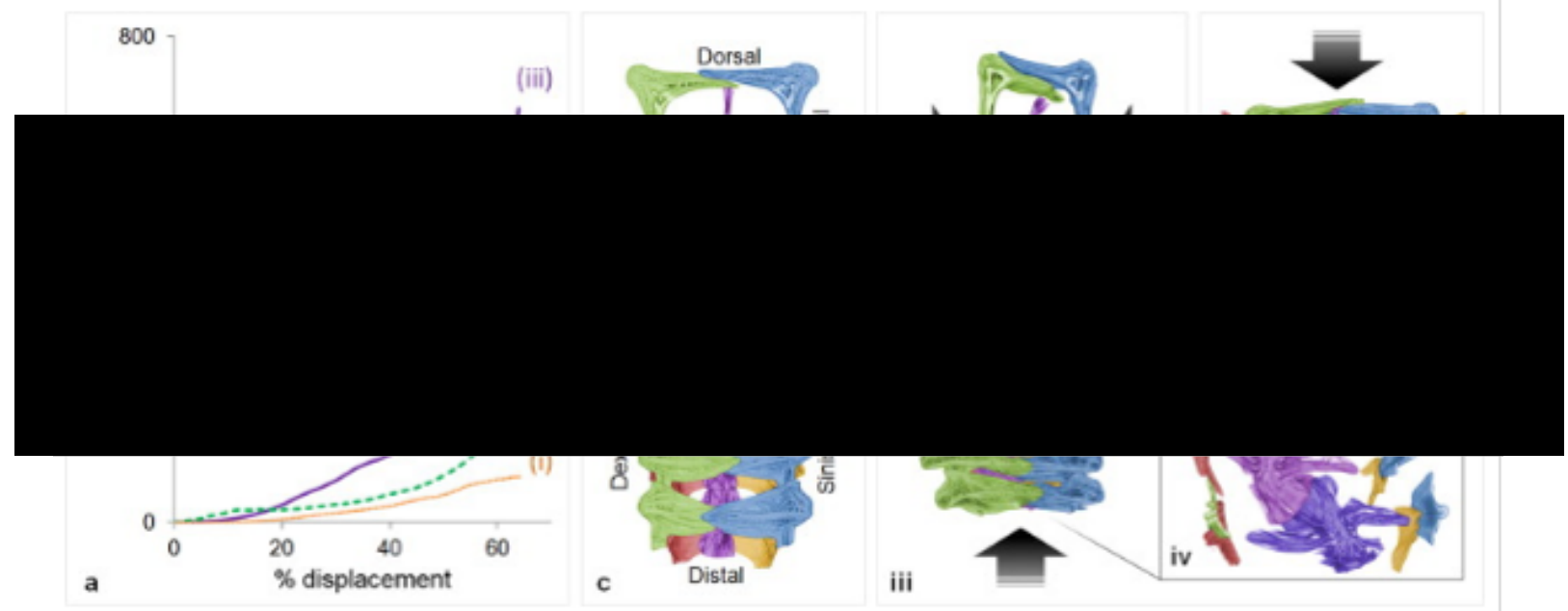

Figure 2.15: Seahorse tail sections under compressive load [113]. (a) Force displacement curves of three different tail sections compressed to $\sim 60 \%$ : (a.i) laterally, (a.ii) ventraldorsally and (a.iii) distal proximally. (b) Distal view of tail cross-section before compression, (c) dorsal view of tail before compression, (i) distal view of tail compressed laterally, (ii) distal view of tail compressed ventral-dorsally, (iii) dorsal view of tail compressed distalproximally, (iv) magnified cutaway of images (iii) showing vertebrae bending due to distalproximal loading.

\subsubsection{D imaging}

The non-invasive nature of $\mathrm{CT}$ has been exploited to follow degradation of synthetic materials $[90,117]$. The nature of using X-ray time-lapse imaging on biological materials offers different challenges to those faced for synthetic ones. As mentioned previously, the environmental conditions can play a significant role in the mechanical and physical properties of biological materials, particularly hydration effects. The level of hydration has been shown to effect the elastic and viscoelastic properties of bone [24] and microstructural properties of skin [118]. In addition, very high doses of X-rays have been shown to progressively degrade the strength, ductility and toughness of bone [119].

A small amount of studies have used 4D imaging as a tool for investigation of biological materials. Examples include the development of a chrysalis during metamorphosis [120] and the dynamics of the flight motor in a living blow fly [121]. However, with advancements being made in in situ test rigs, including temperature and humidity, compression and tension, torsion, nanoindentation and fatigue, it is likely that many more biological materials will be investigated in situ to provide insight into failure modes and links to their mechanical properties. Early studies of these include deformation in bone-periodontal ligament [122], fatigue studies of dental implants [123] and nanoindentation in dentin and tensile deformation in beetle carapace cuticle [124]. 


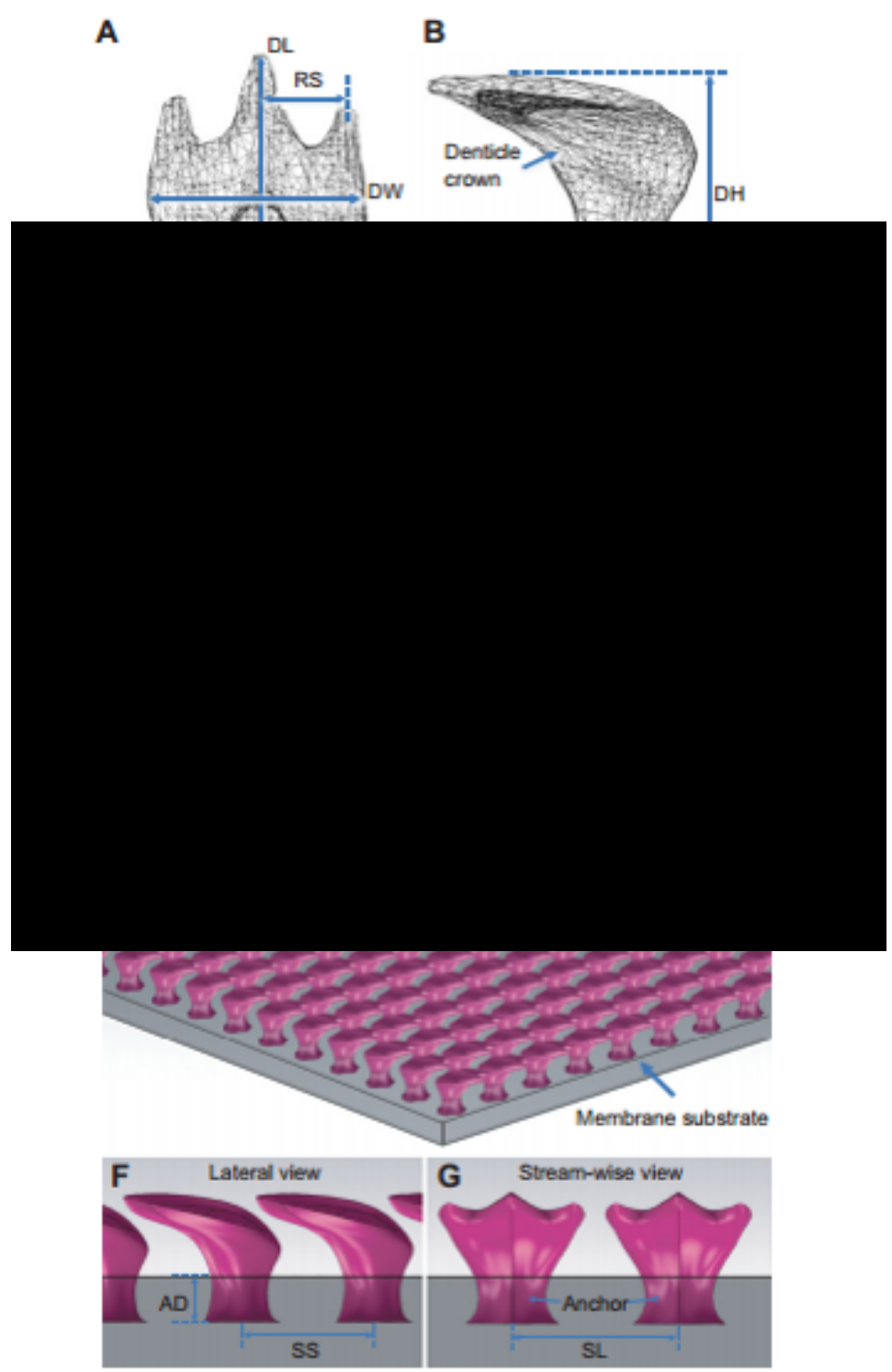

Figure 2.16: 3D reconstructed $\mu \mathrm{CT}$ model of a denticle of a mako shark (Isurus oxyrinchus) [116]. (A-D) mesh, (E-G) 3D prints. 


\subsubsection{Additive layer manufacturing}

Additive layer manufacturing allows for efficient construction of simplified models of complex biological structures in order to investigate their mechanical functions more easily. The models can be created from 3D renderings by visualisation techniques such as X-ray $\mu \mathrm{CT}$. 3D printed structures can provide insight into the relationship between form and function, as they can be mechanically tested. It should be noted that the mechanical properties of materials depend on the feature size, particularly at a sub-micron level [125], therefore the equivalent 3D printed structure may have a dramatically different toughness. Multimaterial 3D printers can be used for these types of investigations as the material properties of the printed structure can be optimised to attempt to replicate similar differences found between organic and inorganic phases in the original structure. Figure 2.17 shows two 3D printed prototypes of a seahorse tail, compression testing was carried out on both of these to determine if the square or circular architecture was better for crushing resistance.

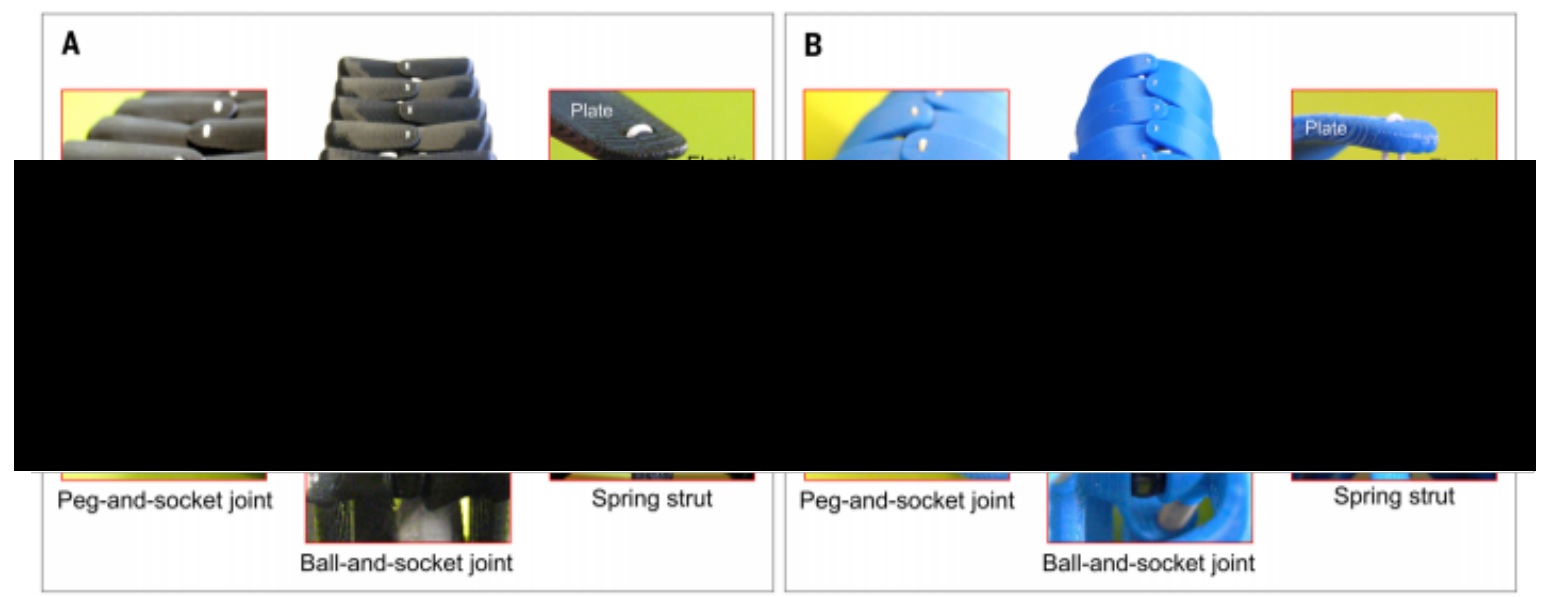

Figure 2.17: 3D printed prototypes designed to mimic the specialised joints of seahorse tail [114]. (A) Square cross section, (B) circular cross section.

\subsection{Summary}

Biological materials are far more complex than synthetic ones, therefore a number of factors must be considered during investigation. In particular, their hierarchical structures and multifunctionality. There are a number of structural design elements which dictate the overall properties of a structure. Biological materials can be studied in many different ways; using a range of equipment spanning across multiple length-scales and under different environmental conditions. Structural, mechanical and chemical analysis is used to characterise biological materials over a range of length scales, and to establish structure-property relationships. 
X-ray microtomography is a powerful technique for the visualisation and analysis of biological materials in 3D. X-ray $\mu \mathrm{CT}$ can provide a wealth of information in the form of density measurements for levels of mineralisation, complete 3D architectures which can be used for additive layer manufacturing or finite element modelling, and analysis of deformation. However, X-ray $\mu \mathrm{CT}$ should be used in correlation with complementary analyses to provide a more complete picture of the structure spanning length scales.

Additive layer manufacturing provides a useful tool for linking form and function using mechanical testing. 


\section{Chapter 3}

\section{Core Methodologies}

This chapter discusses the core methods used in this study. For each method, an overview of the techniques is given, together with its relevance to research within the fields of biological and bioinspired materials. This chapter does not contain all of the methods used in this project, but aims to highlight the central techniques. Those methodologies which are specific to only one of the specimens covered in this thesis are described in the relevant chapter.

\subsection{X-ray microtomography}

A brief overview of X-ray microtomography $(\mu \mathrm{CT})$ has been given in section 2.2.1. As two different systems were used in this study, more details have been provided below.

\subsubsection{Systems}

All tomographic data used in this study was acquired using the Nikon XT H 225 and Zeiss Xradia Versa $520 \mathrm{CT}$ scanners, at the Advanced Imaging of Materials (AIM) facility at Swansea University. The Nikon XT H 225 microfocus X-ray tomography system is a labbased X-ray system fitted with a 1.3 Megapixel Varian PaxScan 2520 amorphous silicon flat panel digital X-ray imager. The $225 \mathrm{kV}$ microfocus X-ray source has a focal spot size of $3 \mu \mathrm{m}$. The Nikon system has a conventional $\mu \mathrm{CT}$ configuration (Figure 3.1) with a fixed source-to-detector distance.

The spatial resolution of a CT image is principally determined by the number and size of detector elements, size of the X-ray focal spot, and the source-object-detector distances. Geometric magnification $g$ is calculated as:

$$
g=\frac{D_{s d}}{D_{s s}}
$$

where $D_{s d}$ is the source-to-detector distance and $D_{s s}$ is the source-to-sample distance, indicated in Figure 3.1. When data is reconstructed at the same resolution as the acquired data, voxel size $V$ can be calculated as: 


$$
V=\frac{P}{g}
$$

where $P$ is the detector pixel size. It follows that a small source-to-sample distance leads to a smaller voxel size and consequently a higher resolution image.

Conversely, the Zeiss Versa 520 CT scanner has an optics-based configuration. This uses a two-stage magnification allowing resolution at a distance, with no fixed source-to-detector or source-to-sample distances. Total magnification $t$ is calculated as:

$$
t=o g
$$

where $o$ is optical magnification. This configuration also allows for phase contrast imaging in addition to absorption contrast imaging. The Versa scanner is equipped with a $160 \mathrm{kV}$ $\mathrm{X}$-ray source and has a spatial resolution of $0.7 \mu \mathrm{m}$.

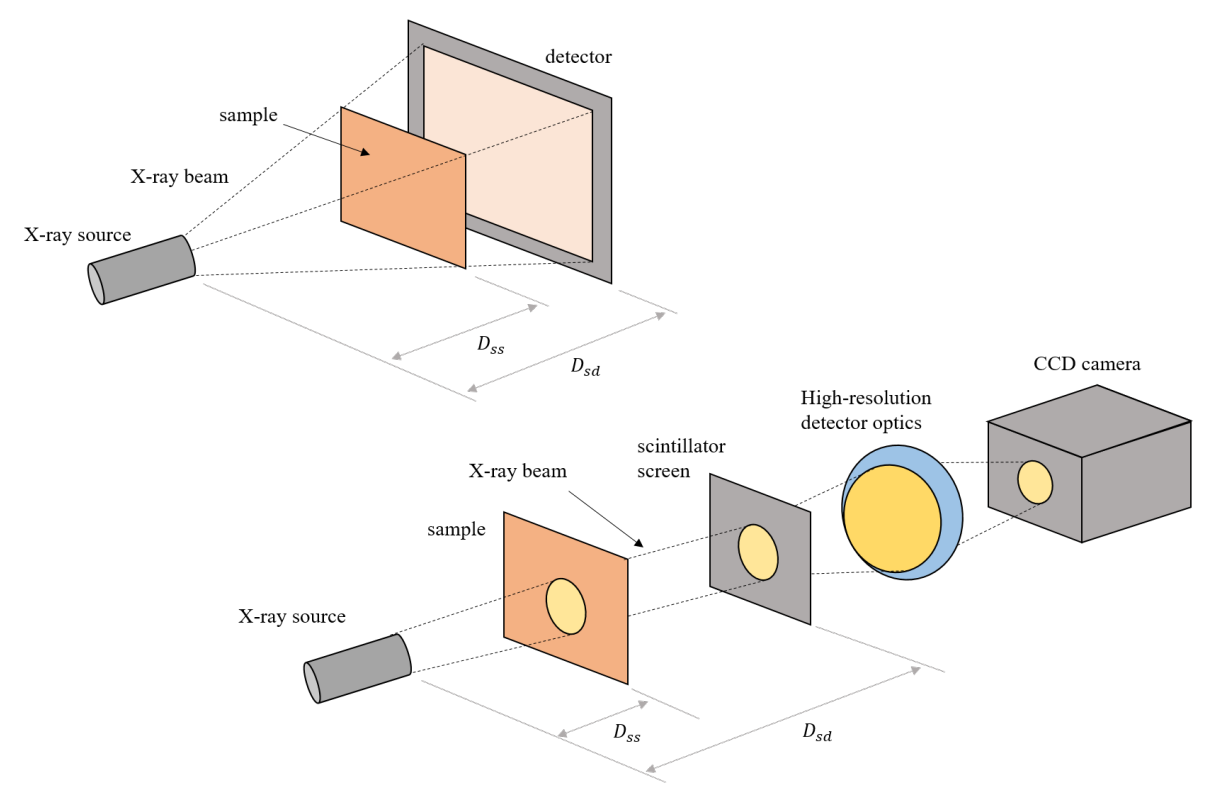

Figure 3.1: Schematic of a conventional $\mu \mathrm{CT}$ (top) and optics-based $\mu \mathrm{CT}$ system (bottom).

\subsubsection{Optimisation of CT scanning}

An iterative process of trial and improvement is involved to produce CT scans of a desired quality, dependent on the sample and its size. Both the mounting of samples and the selection of scanning parameters are part of this process. Samples need to be mounted in a suitable way: to ensure that the specimen does not move during rotation, i.e. from not being securely fixed or from shrinkage due to dehydration, as this would cause blurring in the reconstructed volume; the entire region of interest is captured during rotation so the volume is able to be reconstructed; the samples are not damaged during mounting; and the 
size of the sample allows a suitable voxel resolution to capture the features of interest in appropriate detail.

The outcome of CT scans themselves are determined by the selection of several scanning parameters. Parameters include the X-ray voltage and current (power), X-ray target material, number of projections, exposure time, X-ray filters and voxel resolution. The Xray beam settings are optimised to produce a high level of contrast to distinguish between materials, whilst reducing any artefacts arising from the sample, such as beam hardening. These are discussed in more detail in section 2.2.1.

To achieve optimum results for each hero shrew and cuttlebone specimen scan, 2D radiographs were taken at multiple angles to optimise the scanning parameters and check that the regions of interest would be fully captured. Short, preliminary scans were performed to assess whether the scanning parameters and sample mounting provided satisfactory reconstructed volumes. The process was repeated until the optimal parameters were found for each sample and full scans were then performed. Details of scanning parameters for each scanned specimen are given in the relevant chapters.

Figure 3.2 illustrates several examples of difficulties encountered during the scanning process for hero shrew and cuttlebone samples (see Chapters 4 and 5 respectively for sample information). The metal rod in the re-articulated skeleton of hero shrew (Figure 3.2 (a)) caused streaking artefacts. These were minimised by adjusting the X-ray beam through the use of filters. Figures 3.2 (b) and (c) show damage to cuttlebone chambers and pillars as a result of taking a core sample using a cylindrical tube. In (b), the top two chambers have broken away from the rest of the sample, and in (c) there is clear damage to the edge of the sample and some individual pillars. These issues are partly due to the brittle nature of the cuttlebone and also the method used to core the sample. To overcome these issues, larger sections of cuttlebone were selected and virtual cores were analysed using region of interest scanning techniques. The carbon fibre coring tool was also sharpened to cause less damage to initial chambers and to the edges of the core. Figure 3.2 (d) shows a 2D radiograph of an early scan of a lateral edge of cuttlebone including the dorsal shield. It is clear in this image that the voxel resolution is not high enough to capture individual pillar / chamber detail (see Chapter 5). Also, the settings that allow the X-rays to pass through the shield have as a result reduced contrast across the remainder of the cuttlebone. To produce higher resolution scans of large areas, multiple smaller regions were scanned at a higher resolution and the resulting datasets were then stitched together by aligning the datasets.

\subsubsection{Reconstruction and data visualisation}

Acquired datasets from the Nikon XT H 225 and Zeiss Versa 520 CT scanners were reconstructed in CTPro and XMReconstructor software respectively. Both employ a filtered back-projection algorithm, and corrections for beam hardening and other CT artefacts could be applied at this stage.

A range of commercial and freely-available software was used for the 3D visualisation of 


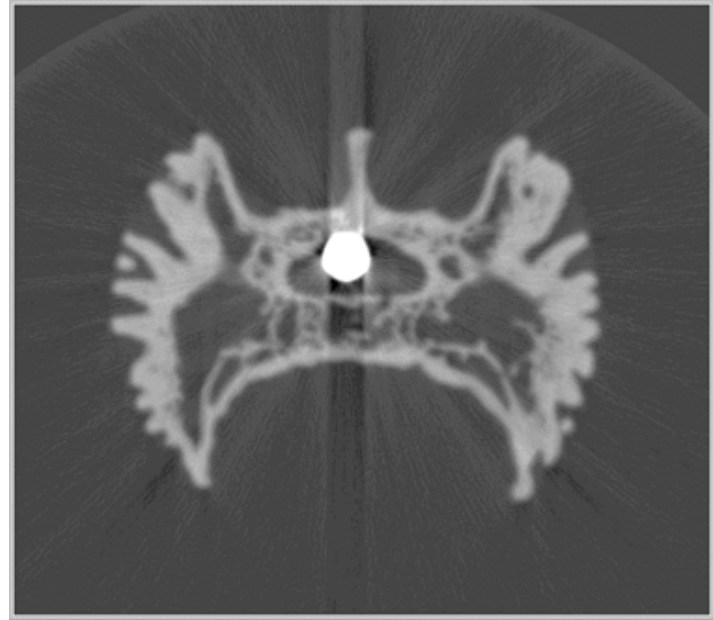

(a)

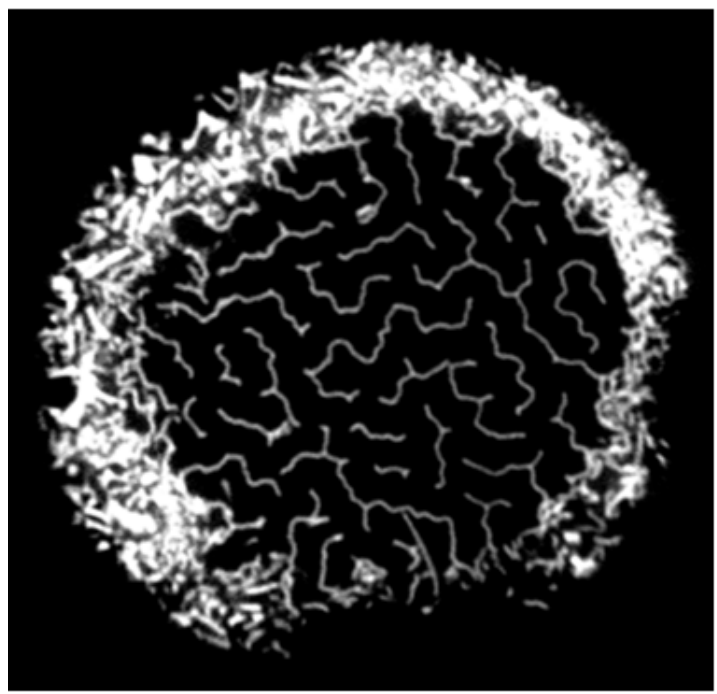

(c)

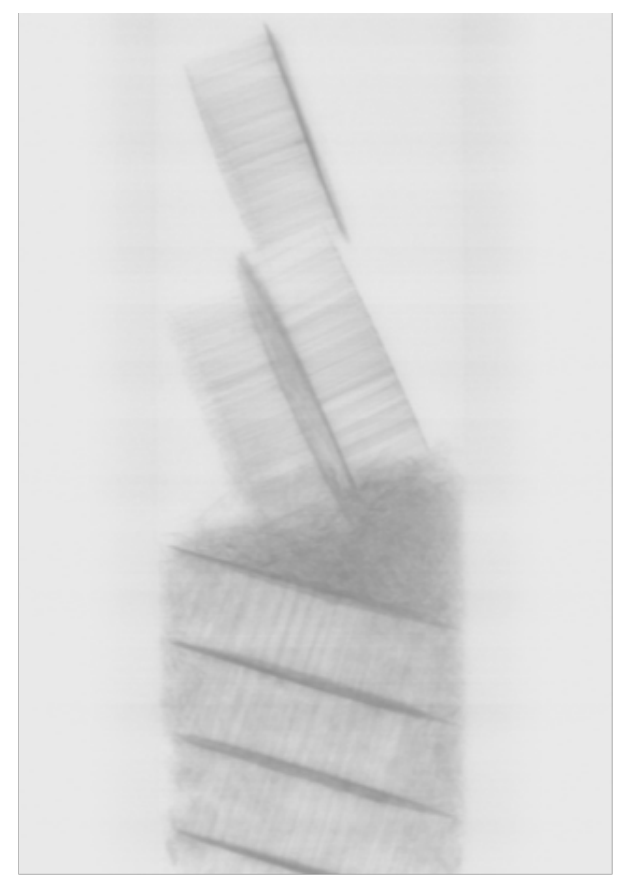

(b)

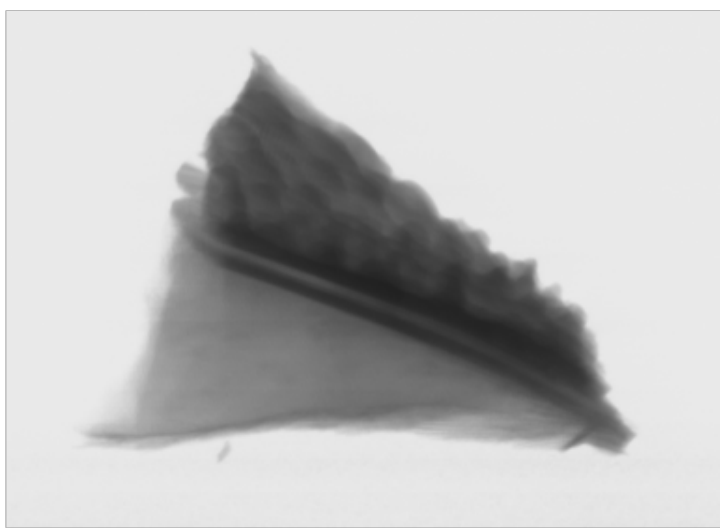

(d)

Figure 3.2: Examples of difficulties encountered when CT scanning: (a) streaking artefacts from metal rod in re-articulated hero shrew specimen; (b) 2D radiograph showing damage to chambers in a cuttlebone core; (c) slice of reconstructed cuttlebone core with damage to individual pillars, particularly at the edges; (d) 2D radiograph of a lateral edge of cuttlebone with limited resolution and poor contrast. 
tomographic datasets. These included VGStudio MAX, Avizo ${ }^{\circledR}$ Fire and Drishti. Each of these software allows rendering, virtual manipulation and segmentation of the data, whilst providing quantitative measurements and analysis. The software used for visualisation and analysis is stated in the relevant chapters.

ImageJ [126] is a freely available Java-based image processing programme, which can be used to provide quantitative measurements of complex objects. Measurements are conducted by statistical pixel analysis, where each image can be thresholded to a user-defined pixel value.

\subsection{Scanning Electron Microscopy}

Scanning Electron Microscopy (SEM) images are generated through the detection of secondary and backscattered electrons as they are emitted from a sample that is being bombarded with a highly focused scanning electron beam. Secondary electron (SE) imaging can provide high resolution images of fractured surfaces revealing topography and microstructural arrangement of the sample. Backscattered electron (BSE) imaging provides atomic density characterisation where lighter areas indicate a higher atomic density. This allows for spatial variations in atomic density across the sample to be recognised. It is necessary for the sample to be conductive, either through the deposition of a conductive layer (commonly gold or carbon), or the use of water vapour in an environmental SEM. One challenge of using SEM imaging on biological materials is that it can cause structural damage from localised heating. This is minimised by either increasing the deposition thickness or by using a beam with a lower $\mathrm{kV}$.

\subsection{Energy Dispersive X-ray Spectroscopy}

Energy dispersive X-ray spectroscopy (EDS) is an effective tool used in conjunction with SE imaging (Figure 3.3(a)) to acquire quantitative and qualitative elemental analysis of samples. A beam of electrons is focused at a sample surface, causing electrons within sample atoms to alter energy states, resulting in the emission of an X-ray to restore the atom to its original energy state. X-rays are detected by the EDS X-ray detector system and identified.

Sample analysis is carried out on polished, conductive samples to ensure an optimum number of X-ray counts are produced. Elemental spectra (Figure 3.3(c)) can be obtained through spot, line or area analysis, where the quantity of the element present is recorded in weight percentages. Elemental mapping (Figure 3.3(b)) can also be used to provide a spatial distribution of the elements within a sample region. Maps are constructed by assigning individual elements a colour where the intensity of the colour varies with the concentration of the present element.

EDS was used in this project to correlate with findings from other observations and results. 


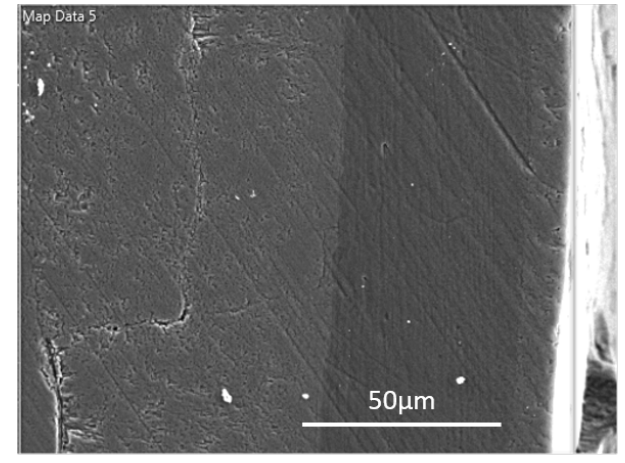

(a)

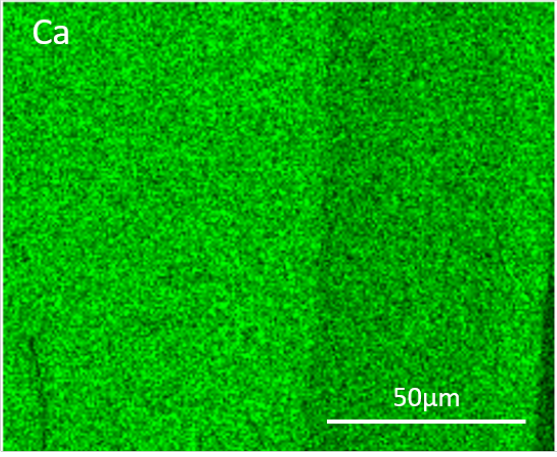

(b)

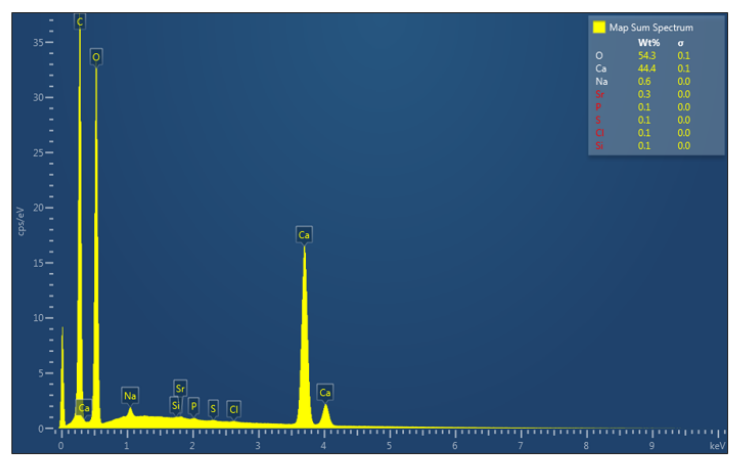

(c)

Figure 3.3: Representative example of EDS elemental mapping and the spectrum of elements identified. A SEM image (a) of the selected region for elemental analysis. A map of calcium (b) is produced for the area along with all elements identified, shown as spectra (c). 


\subsection{Electron backscatter diffraction analysis}

Electron backscatter diffraction (EBSD) is a sophisticated technique used to obtain crystallographic information and phase type of the microstructure of a sample. Following the pioneering work by Nishikawa and Kikuchi $[127,128]$, EBSD has been developed and refined into an automated, in situ analysis technique [129, 130, 131]. The technique is traditionally used in materials science and metallurgy [132], and increasingly in such areas as structural geology [133] and palaeontology [134]. More recently, EBSD has become established in the field of biomineral research for the investigation of biominerals and the biomineralisation process $[135,136,137]$.

\subsubsection{EBSD technique}

EBSD analysis is conducted in a SEM under vacuum with a specialised camera integrated with a phosphor screen detector. The sample must be crystalline, highly polished and free of topography. The sample is tilted at $70^{\circ}$ relative to the electron beam. The electron beam is diffracted from the lattice planes near the sample surface with the diffracted electrons backscattered to the EBSD camera, where they form Kikuchi bands (see Figure 3.4).

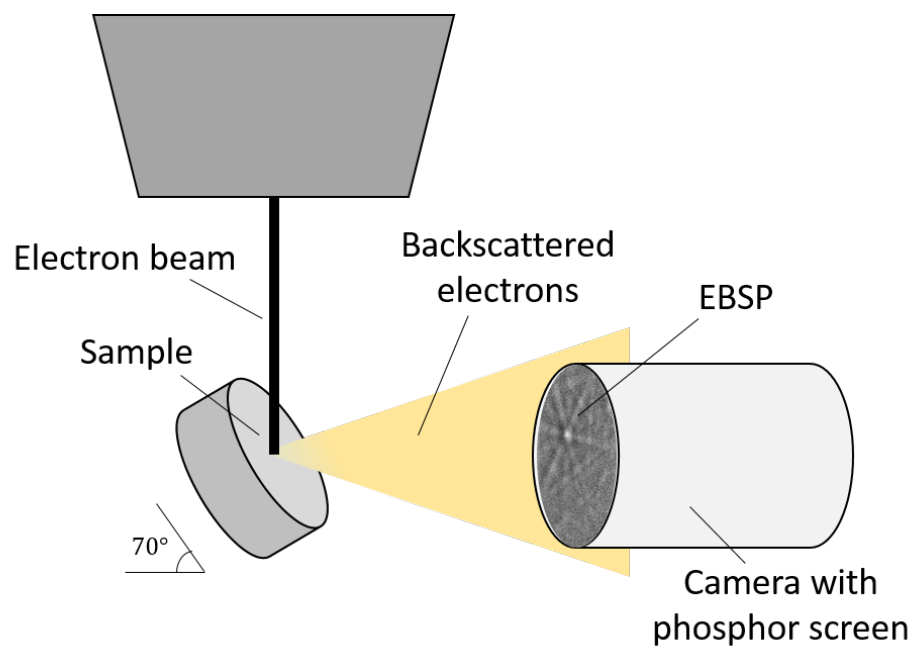

Figure 3.4: Set-up for EBSD analysis. A polished, conductive sample is tilted at $70^{\circ}$ relative to the electron beam. Beam electrons are directed at the tilted sample, where they are diffracted by the crystallographic planes in the sample and imaged using a camera with a phosphor screen.

The Kikuchi bands create an electron backscattered pattern (EBSP) that is unique to the crystal lattice in a specific orientation at that analysis point. By acquiring patterns at each data point, maps can be constructed of crystallographic orientation, texture and mineral phase. For optimum results, the step size must be smaller than the size of the smallest features in order to allow accurate detection of all crystals in a sample. 


\subsubsection{Preparation of samples for EBSD}

An essential requirement for the EBSD of biominerals is thorough sample preparation. In order to achieve high pattern quality, samples must be free of topography and surface damage. The recommended steps for sample preparation of biominerals for EBSD analysis are [138]:

1. Cut sample whilst minimising any deformation caused by heating and vibration.

2. Mount in epoxy resin, with any excess removed after step 3.

3. Grind specimen using the following grit papers for 3 minutes each - P180 $(82 \mu \mathrm{m})$, P320 $(46 \mu m)$, P800 $(21 \mu m)$, P1200 $(15 \mu m)$, P2500 $(8 \mu m)$ and finally P4000 $(<5$ $\mu \mathrm{m})$ for 5 minutes.

4. Polish using alpha aluminium oxide at $1 \mu \mathrm{m}$ and $0.3 \mu \mathrm{m}$, with a final polish using $0.06 \mu \mathrm{m}$ colloidal silica for 5 minutes.

5. Clean in a sonicating water bath and leave to air dry.

The colloidal silica stage is essential for analysis as it removes any surface layers with residual damage, caused by local stress and deformity from harder compound grinding and polishing [139].

Since carbonate materials are insulators, they must be adequately coated with a conductive layer in order to avoid charging, whilst minimising loss of the backscattered signal. Carbon coating is commonly used for this, however the thickness of the coating is critical to produce high quality EBSPs [140].

Silver paint is applied to the edges of the sample and used as a strong adhesive between the sample block and aluminium stub to provide a pathway for charge dissipation during analysis.

\subsubsection{EBSD data analysis and representation}

From the positions of the Kikuchi bands, EBSD analysis software assigns a solution to the diffraction pattern and calculates the corresponding crystal orientation. In order to obtain accurate data, band detection uses sophisticated algorithms to determine which bands are best used in the indexing. For a high validated hit rate without generating wrong solutions, 'Class Indexing' is used creating a more robust process. The reliability of patterns can vary due to inconsistent topography of the sample and presence of organic matter.

EBSD datasets can be viewed and analysed in a number ways. Crystallographic orientation can be displayed using Inverse Pole Figure (IPF) maps, where the colour scheme is designed by assigning a colour to each of the corners of the inverse pole figure, as shown in Figure 3.5. For each map, a reference sample direction is chosen (e.g. $z$ ) and the colour is assigned based on the measured crystal direction and the chosen viewing direction. 

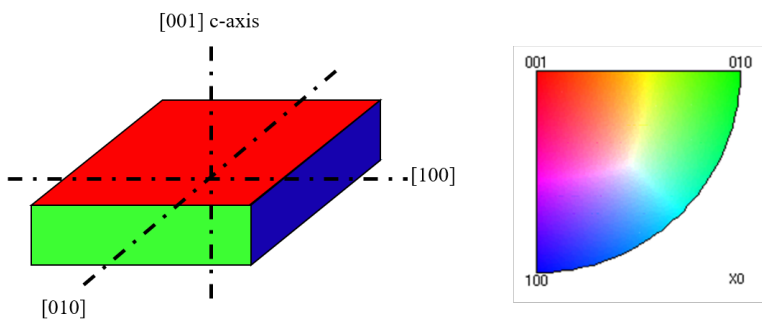

Figure 3.5: Inverse pole figure (IPF) crystal models and colour key for aragonite.

Data can also be viewed as Pole Figures (PF) which represent data in a stereographic projection. They allow $3 \mathrm{D}$ orientation information to be plotted in $2 \mathrm{D}$, which is useful for showing crystallographic orientation in a particular plane or direction. The normals of a crystallographic plane are projected onto a sphere which appear as single points on a hemispheric projection. They can be viewed in $2 \mathrm{D}$ on a circular plane with the chosen axis perpendicular to the field of view. Figure 3.6 demonstrates a crystal in $3 \mathrm{D}$ space and a 2D stereographic projection.

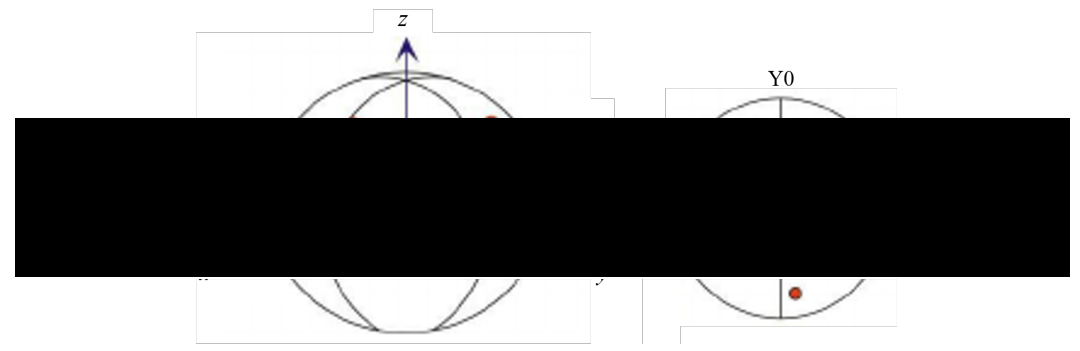

Figure 3.6: Representation of EBSD data as a stereographic pole plot. Modified from image from OIM User's Manual [141].

EBSD analysis has been applied to areas of the cuttlebone specimens. The crystal model of aragonite in Figure 3.5 is the only phase considered in this work.

\subsection{Nanoindentation}

For the mechanical investigation of materials, nanoindentation has become a leading technique [142]. Indentation is conceptually simple; a probe is indented into a sample while measuring the response in the material. For nanoindentation, the dimensions of the equipment are reduced so the mechanical properties can be mapped with a higher spatial resolution. The interaction of the probe and sample is shown in Figure 3.7.

A probe with known mechanical properties and geometry is brought into contact with the sample surface, loaded into the material, and retracted again. During the process three variables are recorded: the applied load $(P)$, displacement $(h)$ and time $(t)$. After 


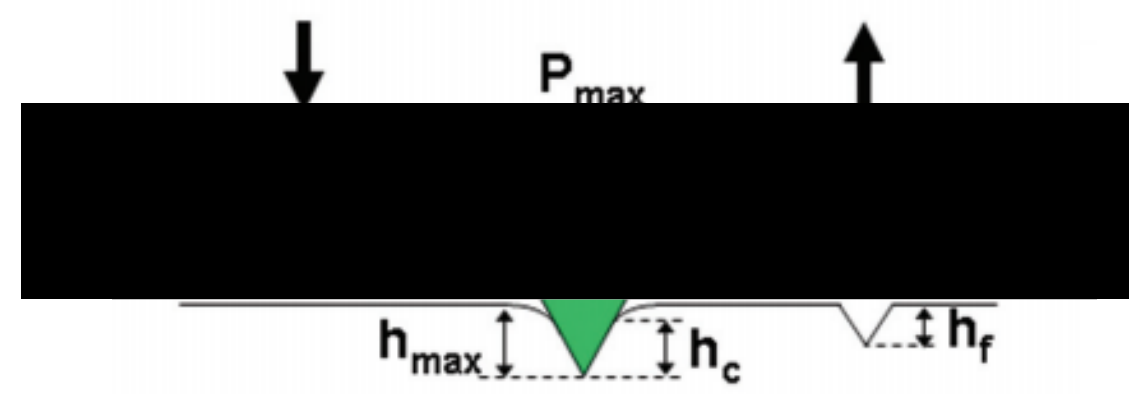

Figure 3.7: Illustration of a nanoindentation measurement. $P_{\max }$ is the maximum load applied; $h_{\max }$ is the penetration depth; $h_{c}$ is the contact depth; $h_{f}$ is the final depth [143].

removal of the probe, the area of indentation is measured. Using this measurement and the load-displacement data, mechanical characteristics, such as hardness, elastic modulus, work hardening behaviour and viscoelastic properties can be calculated [144].

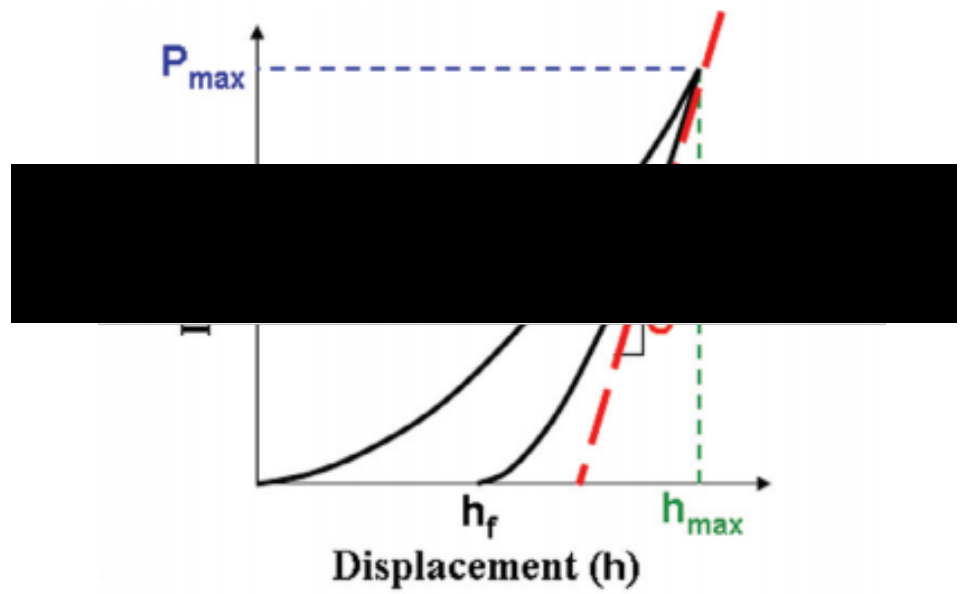

Figure 3.8: Schematic of a typical load-displacement curve. $P_{\max }$ is the maximum load applied; $h_{\max }$ is the penetration depth; $h_{f}$ is the final depth; $S$ is the unloading stiffness [143].

Nanoindentation of biological materials is challenging, as the technique was originally optimised for stiff, linearly elastic and homogeneous materials such as metals and thin films. It offers many advantages over traditional mechanical testing techniques, as biological materials are inhomogeneous and hierarchical, indentation testing can be locally performed [145]. Sample preparation is achieved with relative ease, compared to traditional testing methods for biological methods and is less likely to alter the microstructure. It has been adapted for use on a wide range of biological materials including; stomatopod dactyl club [4], polychaete jaws [146], silks of silkworm and honeybee [147], fish scales [148] and nacre 
tablets [149].

Surface roughness can have an impact on measurements [150], therefore samples for indentation are usually polished, embedded sections. However, the hydration state of samples directly affects the results obtained [143, 151], so it is preferential that biological materials are tested as close to their native state as possible. Limitations in instrumentation can affect this, so experiments are performed on rehydrated specimens, such as by submersion into a liquid prior to indentation. Biomineralised materials, tested in ambient, dry conditions often show constitutive responses not dissimilar to engineering materials (linear elastic-plastic), due to their relatively small water contents and organic fractions [152].

The hierarchical structure of biological materials often results in mechanical properties that vary across the length-scales. By correlating the mechanical data obtained through nanoindentation with local chemical composition measurements or microstructural observations, relationships can be established between observed structure or composition and function of biological materials. Nanoindentation has been utilised to investigate the mechanical properties across the cuttlebone in Sepia officinalis, to complement chemical and structural observations as part of this project.

\subsubsection{Mechanics of nanoindentation}

Using nanoindentation, different mechanical properties can be identified based on constitutive models for the indented materials [152], such as elastic-plastic. Hardness is the resistance to plastic deformation in a material, and the elastic modulus (Young's modulus) measures the resistance to linear elastic deformation and is the stiffness of a material. Nanoindentation hardness $(H)$ is given by:

$$
H=\frac{P_{\max }}{A_{c}}
$$

where $P_{\max }$ is the maximum applied load, $A_{c}$ is the projected area of the indenter at peak load. The contact area can be measured directly (SEM or AFM) or by an analytical approach, termed the compliance method [153, 154]. This method is based on the calibrated relationship between the indentation depth and contact area:

$$
A_{c}=C_{0} h_{c}^{2}+C_{1} h+C_{2} h_{c}^{1 / 2}+C_{3} h_{c}^{1 / 4}+\ldots+C_{8} h_{c}^{1 / 128}
$$

where $h_{c}$ is the contact depth and $C_{n}$ are constants obtained by fitting indents of varying depths in a material with known properties. The contact depth obtained for the elastic response is then used to calculate the contact area. The reduced elastic modulus, $E_{r}$, takes into account that deformation occurs in both the sample and indenter and is given by:

$$
E_{r}=\frac{\sqrt{\pi}}{2} \frac{S}{\sqrt{A_{c}}}
$$

where $S=d P / d h$ is the slope of the initial unloading load-displacement curve (shown 
in Figure 3.8).

The Young's modulus, $E$, can then be obtained from:

$$
\frac{1}{E_{r}}=\frac{1-v^{2}}{E}+\frac{1-v_{i}^{2}}{E_{i}}
$$

where $E_{i}$ and $v_{i}$ are the Young's modulus and Poisson's ratio of the indenter and $v$ is the Poisson's ratio of the tested sample.

\subsection{D printing}

3D printing, also known as additive layer manufacturing, refers to processes used to synthesize a 3D object based on a 3D model. It is an increasingly accessible technology and its popularity can be attributed to its efficient manufacturing process, which is ideal for production of bespoke items at a low cost, as well as its ability to fabricate complex geometries. The models for printing can be constructed directly in computer-aided-design (CAD) software or based on inputs from 3D imaging techniques [155].

There are two main groups of 3D printers commercially available [156]. They are divided into groups that either 'deposit' or 'bind' material. The 'deposit' group delivers material through a nozzle or syringe at set positions to build the object layer-by-layer. The 'bind' group selectively cures or binds materials at localised positions, usually using heat or light to melt or cross-link material to create the 3D object. Both of these groups contain many subgroups, along with the ability to print in a range of materials, such as thermoplastics, ceramics and metals. Work is being done to extend the range and functionality of printable materials, for example tissue engineering [157], electronics [158] and optics [159].

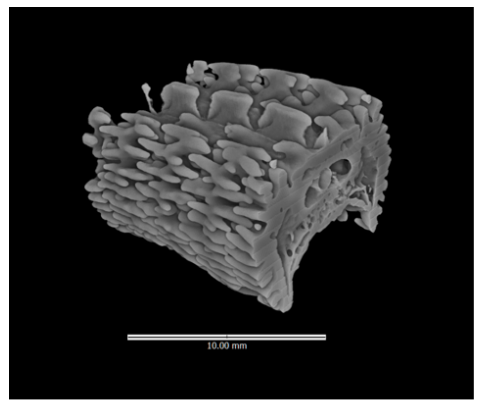

(a)

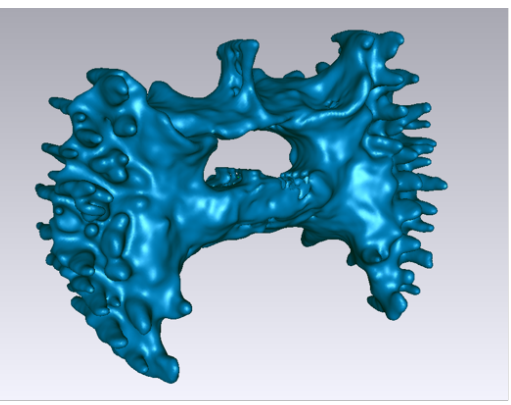

(b)

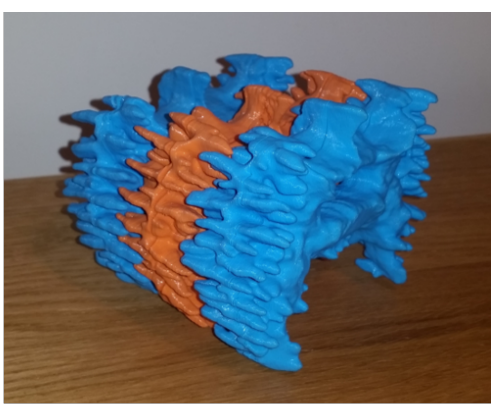

(c)

Figure 3.9: An example of the process of fabricating 3D printed structures. CT data (a) of vertebrae from Scutisorex somereni. Individual vertebrae are segmented and a .STL file (b) is created. From this file, the structure can be scaled-up and fabricated (c) with the process repeated for multiple vertebrae.

Within the context of biological and bioinspired materials, 3D printing is emerging as a promising tool $[160,161]$ as it allows for the fabrication of functional materials based on 
complex architectures inspired by biological systems. The fabrication of these models allows us to better understand these designs and help establish relationships between structural features and their function. Examples of this include: the study of shape on the design principles of the seahorse tail [114], the hydrodynamic function of shark skin [116], spider web geometries [162] and layered composites [163]. These studies use 3D printing to replicate structures that can be mechanically tested directly and therefore reproducibly. It can also be implemented in the manufacturing processes of bioinspired materials, such as the orientation control of anisotropic elements in composite materials [164]. In addition to the replication and production of structures inspired by natural materials, 3D printing is also useful for creating customised mounts and devices for testing of biological materials. As 3D printing technologies improve, along with the ability to print multiple materials at high resolutions, it is an excellent tool for the emulation of natural materials' geometry and material composition.

3D printing has been exploited during this project in two ways: to enable a tactile approach to analysis and visualisation of scaled up samples (Figure 3.9), and for the fabrication of custom rigs. 


\section{Chapter 4}

\section{Vertebrae of Hero Shrew}

\subsection{Introduction and previous work}

This chapter investigates the unique vertebral column of Scutisorex somereni (Thomas, 1910), more commonly known as the hero, or armoured shrew. This animal is found in the forest belt of Africa (southwestern Uganda, eastern Zaire and northern Rwanda) [165] and whilst exact habitat preferences are unknown, it has been found in wet, waterlogged lowland forest with trees, palms and dense undergrowth. A sketch of the hero shew and its skeleton is illustrated in Figure 4.1.

The hero shrew has a typical body length of $12-15 \mathrm{~cm}$, tail length of $6.8-9.5 \mathrm{~cm}$ and weighs between 30 and 115 g. Historically, it was considered a large African shrew, and not until its skeleton was examined did it appear remarkable. The spine has been described as a 'large bony buttress' [166] and is one of the most modified amongst the vertebrates [167]. The spinal column consists of corrugated interlocking vertebrae, as shown in Figure 4.2 in comparison with the spine of an African giant shrew.

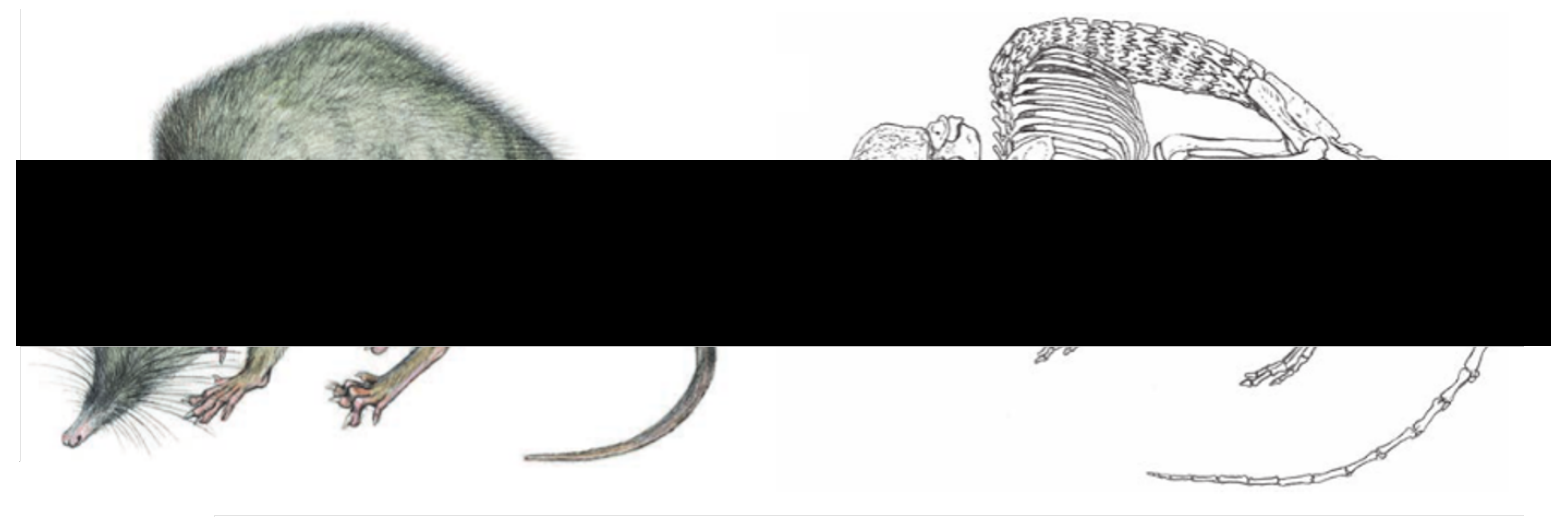

Figure 4.1: Sketch of Scutisorex somereni and its skeleton [165].

The hero shrew was first described by Allen in 1917 [169]. The morphogenesis of the lumbar vertebrae was investigated by Ahmed and Klima [170] who showed that development 


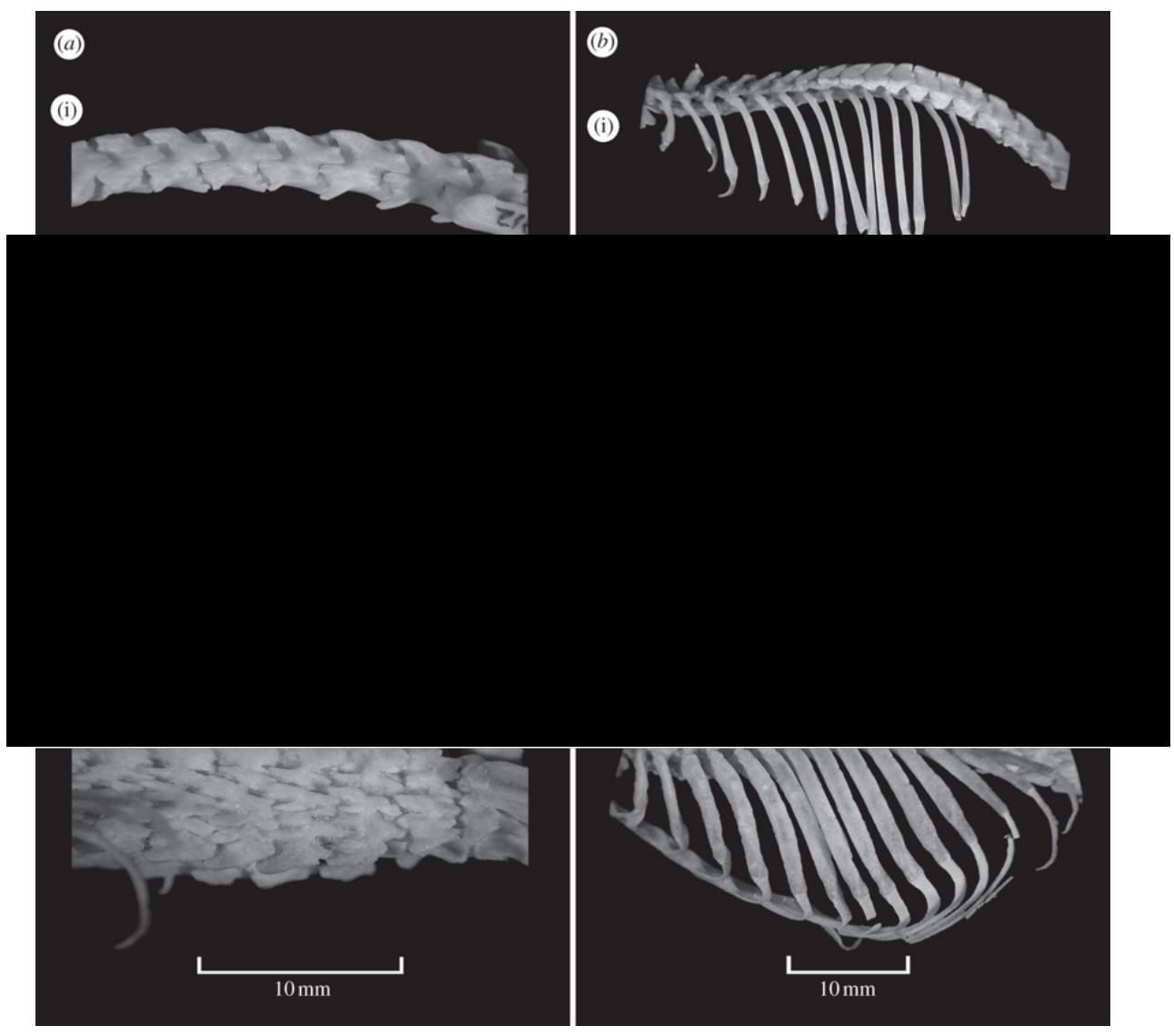

Figure 4.2: Lateral views of (a) lumbar region, and (b) thoracic region, of (i) African giant shrew (Crocidura olivieri), (ii) hero shrew (Scutisorex somereni), and (iii) Thor's hero shrew (Scutisorex thori). Image taken from [168]. 
of tubercles was already evident in early embryonic stages. Little other original work was completed until Cullinane et al. in 1998, who investigated the skeletal scaling relationships [171], spinal musculature [172] and mechanical behaviour of the intervertebral joint [173].

The hero shrew spine is made up of 7 cervical, 14 thoracic, 11 lumbar and 5 sacral vertebrae, as indicated in Figure 4.3. This is consistent with the spinal composition of other mammals apart from the lumbar count, where most mammals normally only have five lumbar vertebrae. The unique features of these vertebrae include an increase in size and diameter, and a profuse development of tubercles (also called spines, processes or apophyses in the literature) mainly on the sides of each vertebrae [169]. These tubercles point in cranial and caudal directions and interlock with tubercles on adjacent vertebrae, forming a basketlike structure. These modifications of the vertebrae appear from the mid-thoracic region to the last lumbar vertebrae, and are particularly prominent in the mid-lumbar region. However, the cervical vertebrae are similar in structure to those in other mammals [165].

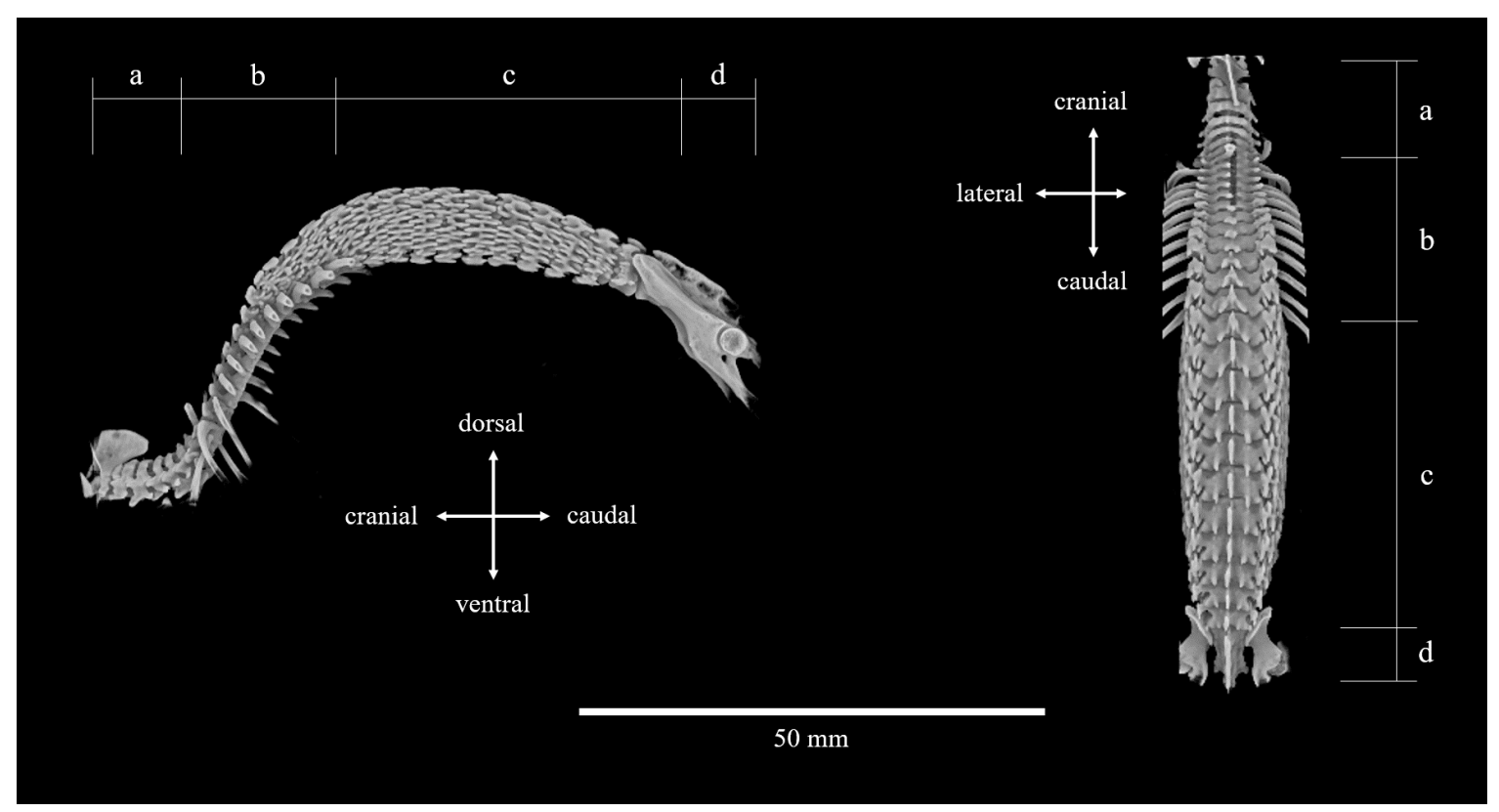

Figure 4.3: Lateral and dorsal views of hero shrew spine with anatomical directions. Vertebral regions indicated by; (a) cervical, (b) thoracic, (c) lumbar, (d) sacral.

The spine accounts for almost $4 \%$ of the shrew's body weight. In contrast, the percentage of overall mass for 12 other comparable small mammals ranged from 0.69 - 1.58\% [171]. In addition to the vertebral modifications, the ribs have also been shown to be more robust with a larger diameter than those on similar small mammals [171]. However, the appendicular skeleton does not show any significant modifications.

To compensate for this unique spinal column, the muscles surrounding it are also modified. The transverse muscles are reduced in comparison with the American short-tailed shrews Blarina and rats Rattus, whilst muscles used to extend and flex the spine are well 
developed. This causes the shrew to walk in a snake-like fashion [172].

A combination of the additional lumbar vertebrae and interlocking tubercles, along with general increased size and mass, allows the hero shrew spine to resist large compressive forces and axial torsion whilst protecting the animal's organs. It has been said anecdotally that the shrew can take the full weight of a man on its back (around $70 \mathrm{~kg}$ ) [174], roughly 1,000 times the shrew's weight. The additional vertebrae lead to more intervertebral joints, therefore increasing the flexibility of the spinal column to the extent that the shrew can turn its body through $180^{\circ}[172]$. It has also been shown that the intervertebral joint of the hero shrew exhibits the mechanical behaviour of a rigid construct, rather than a viscoelastic joint under axial compression [173], as it displays no creep relaxation behaviour.

A sister species, Scutisorex thori (Thor's hero shrew), was discovered in the Republic of Congo and also exhibits a similar unusual spinal morphology [168]. This shrew has 8 lumbar vertebrae with smaller tubercles than those found in the hero shrew. It has been hypothesised that this species provides an evolutionary link between the hero shrew and other shrews [168].

It has been concluded that there is no known satisfactory ecological, behavioural or functional explanation for the hero shrew's unique vertebral morphology. One recent hypothesis is that it allows the animal to push itself under logs and between palm leaves and trunks to find food using its spine as leverage [168]. However, this has been difficult to prove due to limited behavioural observations in its natural habitat.

The objective of this chapter is to characterise this unique morphology through the use of $\mathrm{X}$-ray microcomputed tomography. There are very limited numbers of complete hero shrew specimens available to study, therefore it is imperative that analysis of these specimens is non-destructive. 2D radiographs of hero shrew have previously been taken whilst in spinal extension and flexion, highlighting the interdigitation of tubercles [171, 173]. However, information gained from 2D radiographs is limited to a single field of view and individual components are difficult to distinguish, particularly given the complex three dimensionality of the interlocking vertebrae. The use of X-ray $\mu \mathrm{CT}$ overcomes these challenges and provides more information in $3 \mathrm{D}$ at a far higher resolution. Morphological variations along the spinal column are examined, and the interaction between adjacent vertebrae is also considered. Insight into morphology may help to provide information as to why the hero shrew exhibits this vertebral structure, and may also be applied in future engineering design.

\subsection{Experimental methods}

In this section the specimens used in this study are described and the various experimental methods used to characterise the hero shrew spinal structure are discussed. 


\subsubsection{Specimens}

A re-articulated skeleton of Scutisorex somereni was kindly loaned by Dr. Andrew Kitchener from the National Museum of Scotland. A metal rod was used to hold the skeleton together, therefore mounting positions for $\mathrm{CT}$ data acquisition were restricted, and the rod caused some beam hardening and streaking artefacts.

Three samples were generously loaned from Dr. Dennis Cullinane. These samples are indicated in Figure 4.4. It is clear from the figure that the majority of the modified vertebrae have been removed during previous analyses. To ensure the specimens didn't dry out, they were stored in a solution of $70 \%$ ethanol. In addition to the three specimens, Dr. Cullinane also provided histological slides of some sections of the missing vertebrae.

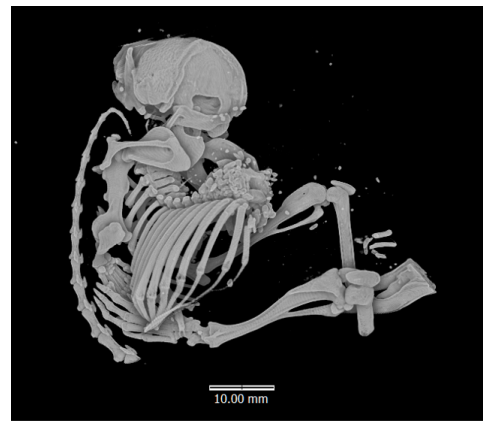

(a)

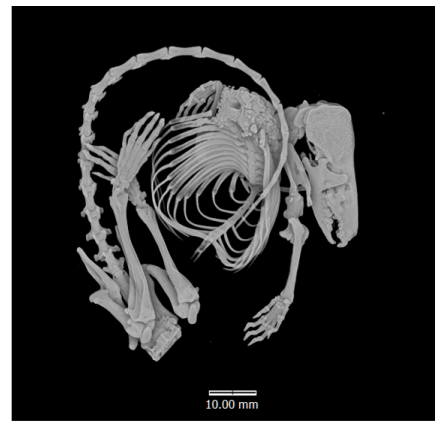

(b)

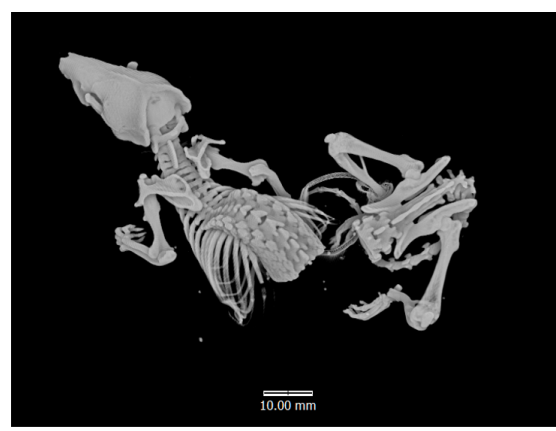

(c)

Figure 4.4: Hero shrew specimens provided by Dr. Cullinane; (a) US_sample1, (b) US_sample2, (c) US_sample3. Note the removal of the lumbar vertebrae in all images. The remaining lumbar vertebrae from (c) were removed and used for higher resolution imaging (US_vert).

\subsubsection{X-ray microtomography}

All tomographic data used in this chapter was acquired using the Nikon XT H 225 and Zeiss Xradia Versa 520 CT scanners at the AIM facility at Swansea University. A large number of scans were performed at various resolutions to achieve a complete picture of the hero shrew. The scanning parameters for those samples used in this study are given in Table 4.1.

To prevent shrinkage and consequential blurring of images during data acquisition, samples US_sample1, 2 and 3 were kept moist with cotton wool soaked in the ethanol solution in sealed plastic containers.

\subsubsection{Segmentation and visualisation}

Vertebrae were considered in isolation in order to investigate their morphology. Therefore, the $3 \mathrm{D}$ reconstructed datasets of the vertebral column were segmented to focus on each vertebra individually. Segmentation of vertebrae was challenging for several reasons: 


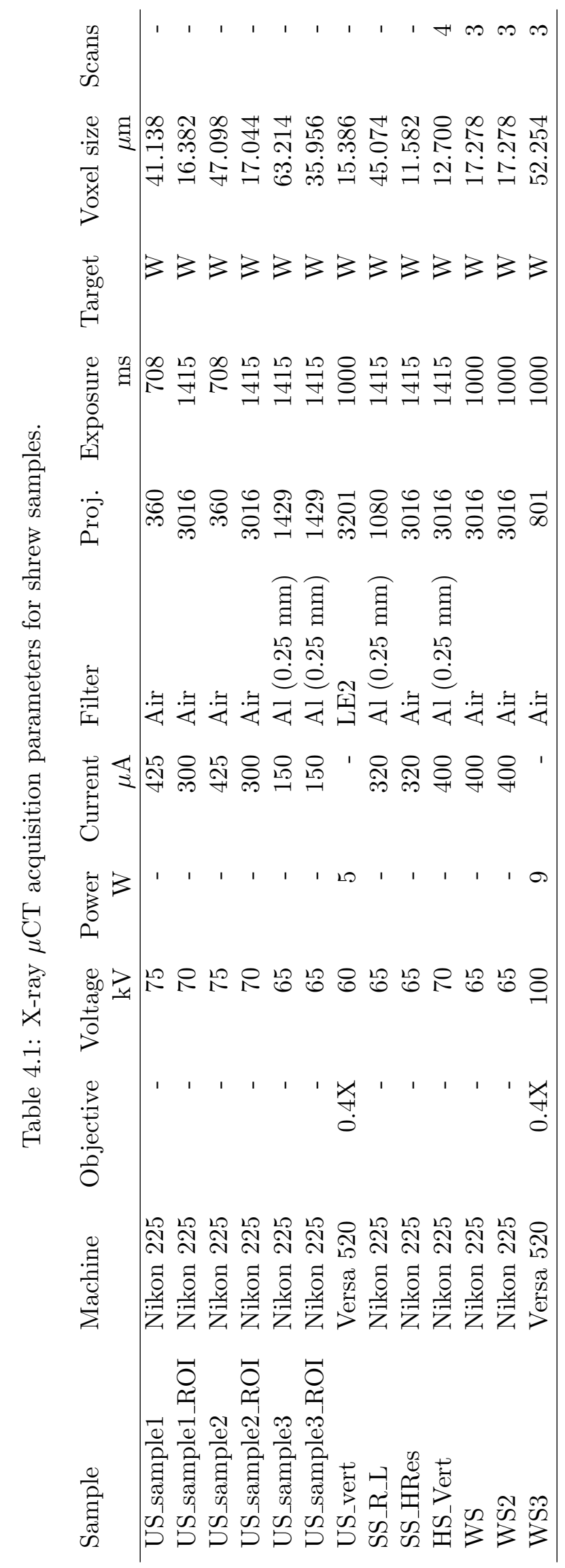


1. Histogram-based thresholding could not separate adjacent vertebrae, as there was minimal change in attenuation.

2. Interlocking tubercles from adjacent vertebrae could not always be distinguished in certain planes.

3. The metal rod in the re-articulated skeleton had to be removed virtually.

It was therefore a labour-intensive, manual process to correctly isolate all vertebrae. After trialling various software packages, including VGStudio Max, Avizo Fire and Drishti, Drishti Paint was found to be the most suitable for segmentation of these specimens. Drishti Paint allows users to manually segment ('tag') and generate surface meshes of regions from $3 \mathrm{D}$ volumes. These meshes are watertight so can be used for further analysis as well as for 3D printing. A combination of graph cut and livewire segmentation techniques were used.

Graph cut segmentation - images are divided into object and background regions. A picture is formed by connecting pairs of neighbouring pixels by weighted edges. The user defines pixels as object or background, and a standard min-cut/max-flow algorithm is used to identify the object region.

Livewire segmentation - a semi-automatic method in which the user defines a starting point, and a lowest cost path algorithm is used to decide a likely path along a high gradient ridge. The user can help guide the path by selecting points along the path until the required region is selected.

Segmented volumes were exported individually as .raw and Drishti specific .pvl.nc files, with the 'tagged' surface and relevant transfer function to capture its internal structure. These volumes were then imported into VGStudio Max and an isosurface was created using a surface determination function to acquire volume and surface area measurements. Tubercle counts were conducted by manually marking individual tubercles on both the cranial and caudal faces.

\subsubsection{Optical microscopy}

Histological slides of the hero shrew vertebrae provided by Dr. Cullinane were examined using optical microscopy. They showed the internal structure of sections of the hero shrew vertebrae and therefore were used for correlation and validation purposes alongside the acquired 3D data from X-ray $\mu \mathrm{CT}$. Images were acquired using a Reichert Jung MeF3 optical microscope.

\subsubsection{D printing and vertebral range-of-movement analysis}

Three digitally-segmented lumbar vertebrae (L4, L5 and L6) from the re-articulated skeleton were meshed, scaled (x10) and 3D printed to give insight into the range of movement between vertebrae. These vertebrae were selected as they represent the middle portion of the lumbar vertebrae and are therefore amongst the most highly modified. 
The meshed vertebrae were cleaned in Netfabb. Any holes were filled and the mesh was decimated to reduce printing time, without impacting the vertebrae morphologies. They were printed with PLA on the Ultimaker ${ }^{2}$.

The range of movement between these vertebrae was analysed by capturing images at maximum deflection in the lateral flexion, extension and flexion movements. The angle of rotation for each was measured digitally using the angle tool in ImageJ.

\subsection{Results and discussion}

The results section first considers the morphological variation in the vertebral column. The re-articulated skeleton is the primary source of the data, however regions of vertebrae from two other samples are also analysed for comparison purposes. The results then look at the interaction between adjacent vertebrae, including the range of movement between vertebrae.

\subsubsection{Vertebrae analysis}

The aim of the analysis of the hero shrew vertebrae is to consider morphology using 3D data from X-ray $\mu \mathrm{CT}$, which has not been done previously. By attempting to further understand the form and unknown function of this unique vertebral structure, novel engineering applications may be realised.

Figure 4.5 shows the segmented vertebrae considered in analysis. Each vertebra is highlighted in a different colour and is labelled based on the type of vertebra and position in its group. The first 3 cervical vertebrae are missing; these vertebrae are located away from the vertebrae of interest in this study, and they have been shown to be similar in structure to those of other mammals [165]. The sacral vertebrae are not included in analysis because the vertebrae are fused and so cannot be segmented. Figure 4.6 highlights the various features found on each lumbar vertebra.

Table 4.2 gives metrics on volume, surface area, total tubercle count, cranial tubercle count and caudal tubercle count for each of the vertebrae labelled in Figure 4.5. Visual representations of volume, surface area and tubercle count are given in Figures 4.7, 4.8 and 4.9 respectively.

Figure 4.7 displays the volumes of each vertebra. Volumes remain low and consistent across the analysed cervical vertebrae and the first seven thoracic vertebrae (T1 - T7). From vertebra $\mathrm{T} 8$, in the caudal direction, volumes increase and decrease smoothly through the rest of the thoracic and lumbar vertebrae. Vertebra L4 has the largest volume of $86.87 \mathrm{~mm}^{3}$, an order of magnitude larger than the volumes of the cranial vertebrae.

Figure 4.8 displays the surface areas of each vertebra. It is clear that there is a very strong positive correlation between surface area and vertebrae volumes displayed in Figure 4.7. The same overall patterns are seen in the surface area as in volume. The most caudal cervical vertebrae, $\mathrm{C} 7$, shows a notable increase of around $25 \%$ in surface area compared with the vertebrae before and after it. This pattern is also evident in the vertebrae volume 


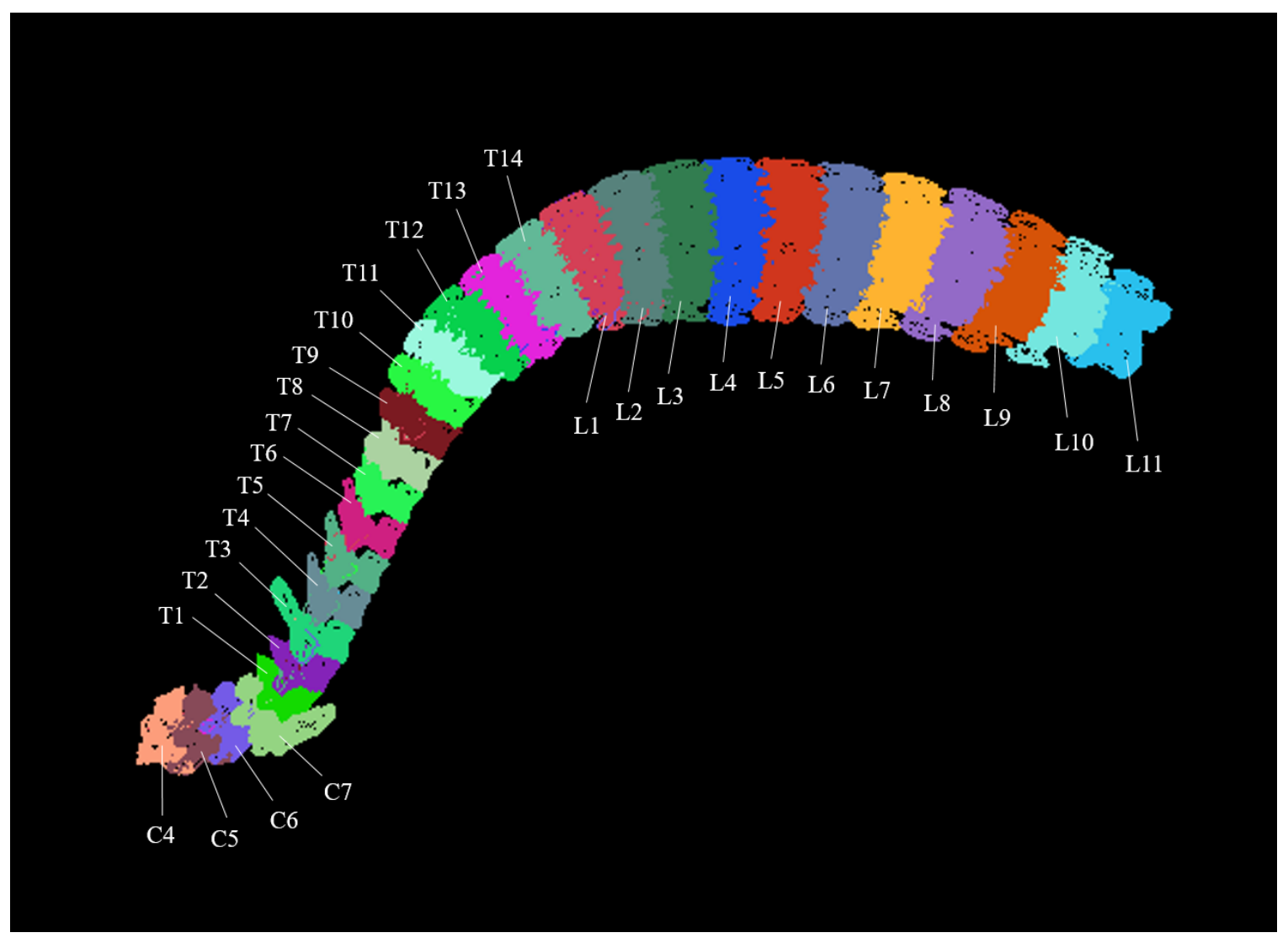

Figure 4.5: Segmented vertebrae used for analysis. C - cervical, T - thoracic, L - lumbar.

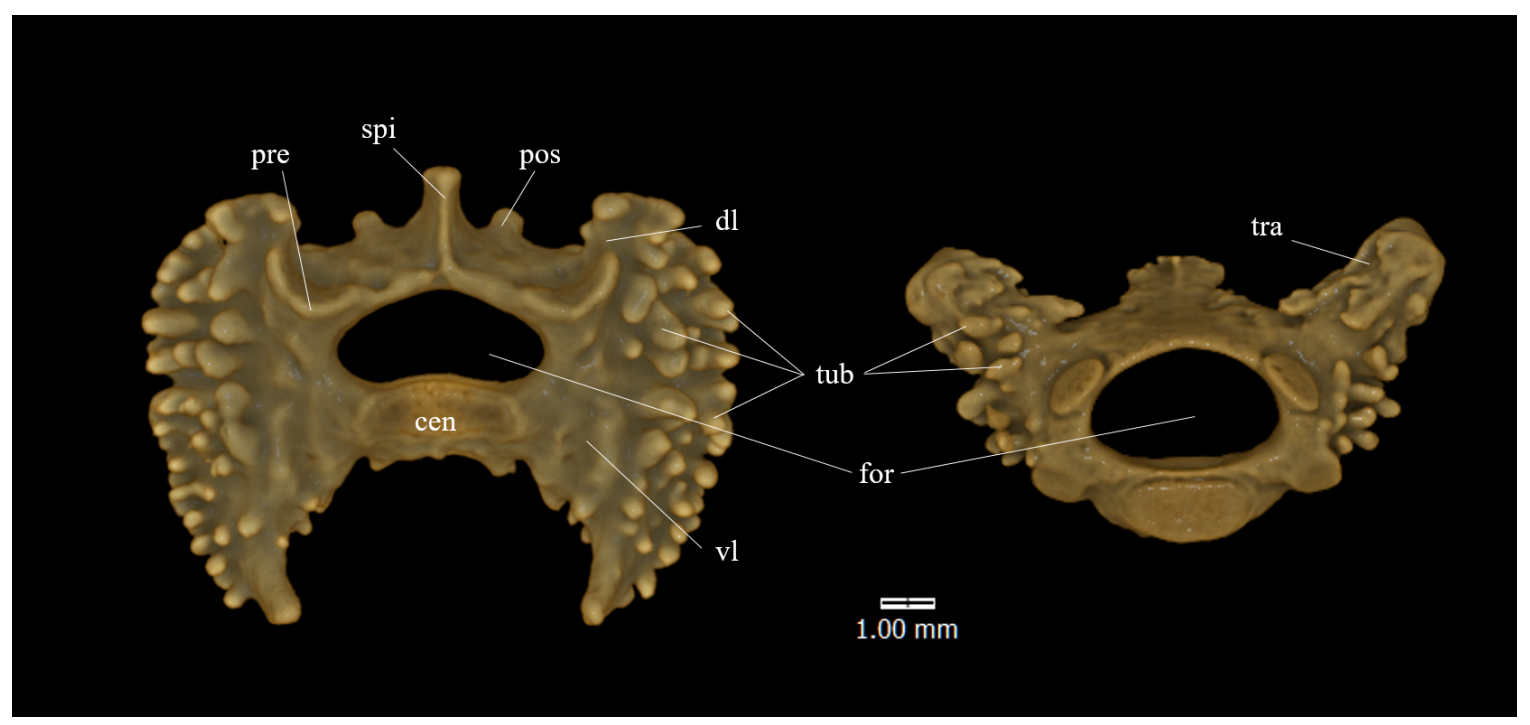

Figure 4.6: Cranial view of vertebra with labels indicating features. pre - prezygapophysis (superior process), spi - spinous process, pos - postzygapophysis (inferior process), cen centrum, dl - dorsolateral part, tub - tubercles, vl - ventrolateral part, tra - transverse process, for - foramen. Modified from [170]. 
Table 4.2: Summary of vertebrae from re-articulated skeleton.

\begin{tabular}{|c|c|c|c|c|c|}
\hline $\begin{array}{l}\text { Vertebrae } \\
\text { ID }\end{array}$ & $\begin{array}{l}\text { Volume } \\
\left(\mathrm{mm}^{3}\right)\end{array}$ & $\begin{array}{r}\text { Surface Area } \\
\left(\mathrm{mm}^{2}\right)\end{array}$ & $\begin{array}{r}\text { Total tubercle } \\
\text { count }\end{array}$ & $\begin{array}{l}\text { Tubercle count } \\
\text { (cranially) }\end{array}$ & $\begin{array}{r}\text { Tubercle count } \\
\text { (caudally) }\end{array}$ \\
\hline $\mathrm{C} 4$ & 11.32 & 74.14 & 9 & 3 & 6 \\
\hline C5 & 11.76 & 82.36 & 10 & 6 & 4 \\
\hline C6 & 10.34 & 69.28 & 12 & 6 & 6 \\
\hline $\mathrm{C} 7$ & 12.75 & 93.61 & 14 & 8 & 6 \\
\hline $\mathrm{T} 1$ & 7.25 & 56.20 & 15 & 9 & 6 \\
\hline $\mathrm{T} 2$ & 8.31 & 61.23 & 8 & 2 & 6 \\
\hline T3 & 8.76 & 68.52 & 8 & 4 & 4 \\
\hline $\mathrm{T} 4$ & 7.21 & 57.56 & 8 & 4 & 4 \\
\hline T5 & 8.73 & 60.84 & 8 & 4 & 4 \\
\hline T6 & 8.20 & 60.71 & 8 & 4 & 4 \\
\hline $\mathrm{T} 7$ & 8.66 & 64.52 & 10 & 4 & 6 \\
\hline $\mathrm{T} 8$ & 10.21 & 77.41 & 12 & 4 & 8 \\
\hline T9 & 13.14 & 95.42 & 20 & 8 & 12 \\
\hline $\mathrm{T} 10$ & 19.46 & 124.14 & 32 & 14 & 18 \\
\hline $\mathrm{T} 11$ & 24.29 & 164.87 & 53 & 25 & 28 \\
\hline T12 & 33.77 & 214.78 & 82 & 36 & 46 \\
\hline T13 & 38.26 & 238.47 & 78 & 31 & 47 \\
\hline T14 & 53.99 & 287.79 & 94 & 39 & 55 \\
\hline L1 & 63.80 & 328.91 & 118 & 56 & 62 \\
\hline L2 & 73.13 & 364.48 & 123 & 58 & 65 \\
\hline L3 & 75.37 & 379.71 & 112 & 56 & 56 \\
\hline L4 & 86.87 & 400.34 & 110 & 48 & 62 \\
\hline L5 & 82.28 & 401.17 & 94 & 43 & 51 \\
\hline L6 & 83.32 & 408.41 & 90 & 39 & 51 \\
\hline L7 & 82.08 & 387.92 & 86 & 42 & 44 \\
\hline L8 & 73.62 & 376.01 & 77 & 35 & 42 \\
\hline L9 & 58.36 & 326.68 & 74 & 37 & 37 \\
\hline L10 & 41.21 & 256.87 & 64 & 28 & 36 \\
\hline L11 & 26.29 & 177.81 & 30 & 18 & 12 \\
\hline
\end{tabular}




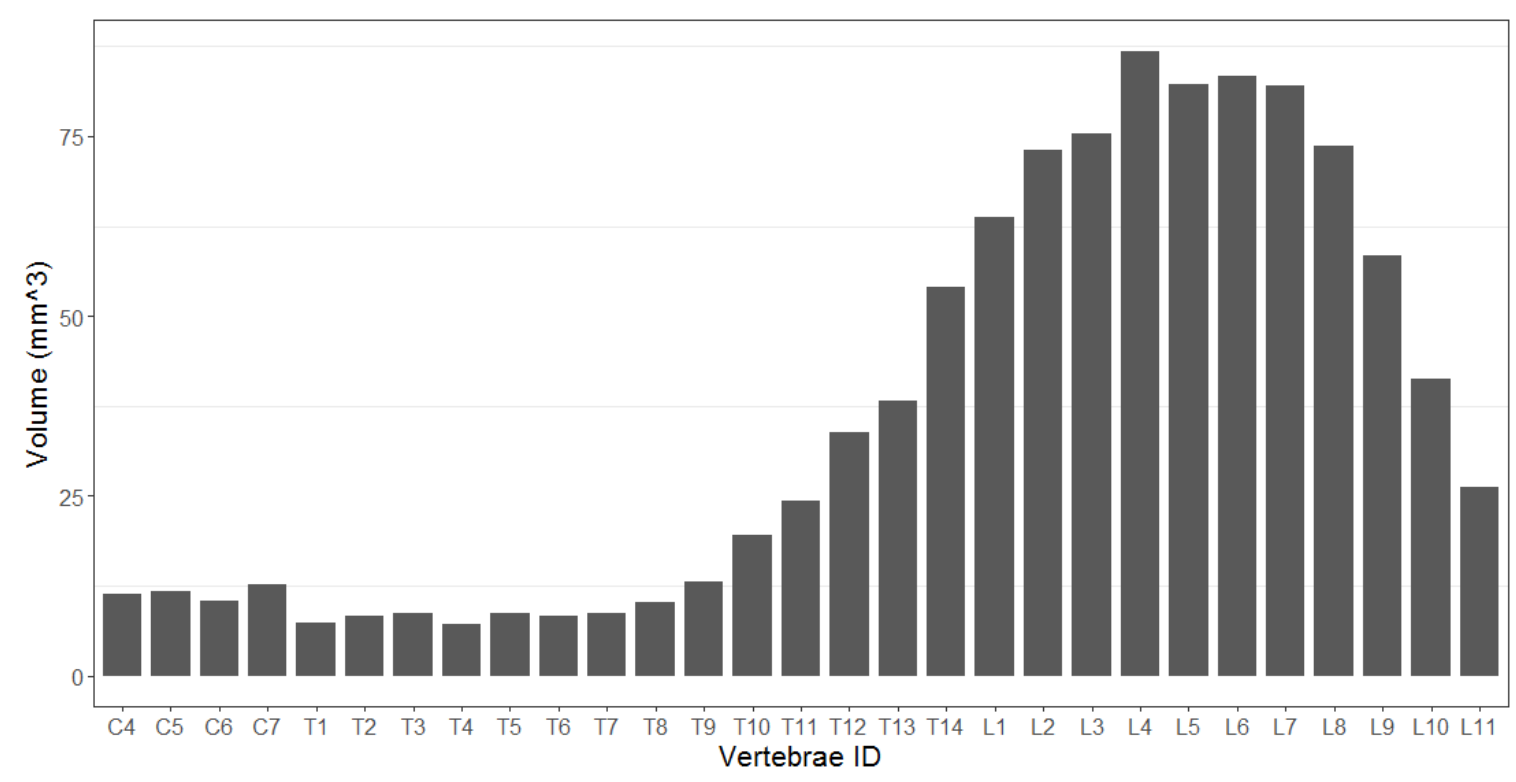

Figure 4.7: Vertebra volume $\left(\mathrm{mm}^{3}\right)$.

distribution. Figure 4.5 shows that $\mathrm{C} 7$ has additional material on the ventral side, which could explain the surface area increase. Vertebra L6 has the largest surface area of 408.41 $\mathrm{mm}^{2}$, marginally more than L4 and L5.

Figure 4.9 displays cranial and caudal tubercle counts for each vertebra, coloured red and blue respectively. The total tubercle count for each vertebra is the sum of the cranial and caudal counts. Total counts are low for vertebrae C4 - T8, having approximately the same number of tubercles on each face. As the vertebra size (volume and surface area) increases through the thoracic and lumbar regions, the tubercle count on the caudal face is predominantly higher than the cranial face. Tubercle counts across vertebrae do not have a distribution as smooth as volume or surface area measurements. For example, T13 has 5 fewer tubercles on its cranial face than T12. Variation in tubercle counts between vertebrae is understandable because tubercles of any size are included in the counts. These variations do not seem to have a notable effect on volume and surface area measurements. The highest number of tubercles is found on vertebra L2. This is interesting since L2 does not have the largest volume (L4) or surface area (L6).

An area of particular interest in this work is the interface between adjacent vertebrae. Specifically, the tubercle counts of the connecting caudal and cranial faces, and how they interact. The interface differences in tubercle counts between a vertebra's cranial face and its preceding caudal face are shown in Figure 4.10. Vertebrae C5 - T7 have low total tubercles counts and so the interface differences are of little interest. Vertebrae T8 - L11, however, have higher numbers of tubercles and the interface tubercle count differences show distinct patterns. Vertebrae in the lumbar region typically have fewer tubercles on each cranial face than the preceding caudal face. The interfaces of T10, T11, T12 and L1 exhibit the opposite 


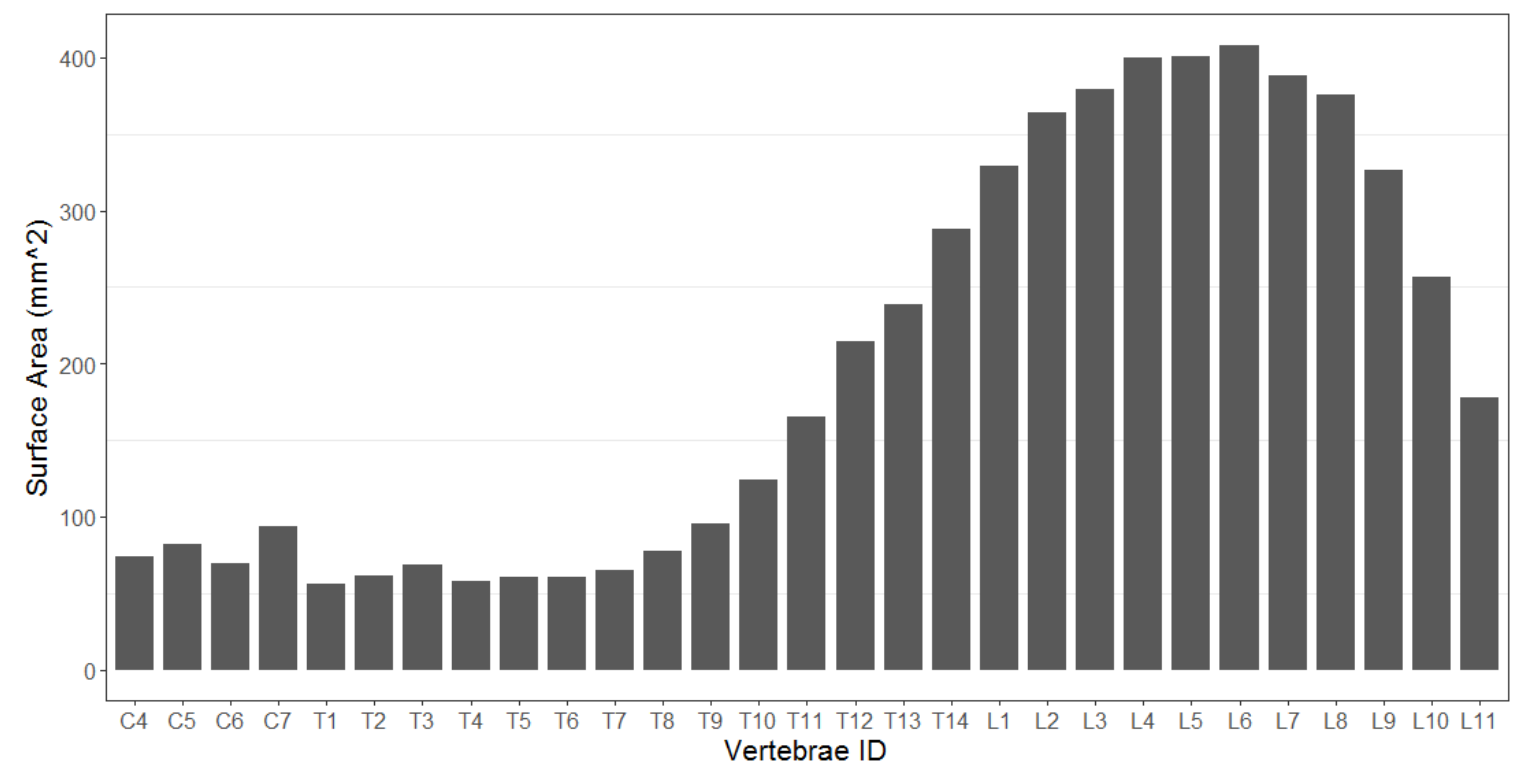

Figure 4.8: Vertebra surface area $\left(\mathrm{mm}^{2}\right)$.

relationship, with more tubercles on the cranial face than the caudal face before them. This relationship is assessed with other samples in section 4.3.2.

As noted from Figure 4.10, the number of tubercles on the caudal face is higher than the cranial face across the lumbar region. Figure 4.11 gives an example of two adjacent vertebrae; L5 and L6. The dashed lines in the image show two lines of tubercles on the caudal face of L5 and one line of tubercles on the cranial face of L5 which interconnect. Close inspection of the images shows three approximate lines of tubercles on each lateral edge of L5 and two lines on each lateral edge of L6. The outer set of tubercles interlace, whilst the inner lines of the cranial face are offset against the lines on the caudal face of the adjoining vertebra to provide a precise fit. This ordered pattern of tubercles is clearly shown in the thoracic and lumbar vertebrae illustrated in Figure 4.12. Later in section 4.3.3 the interaction between these vertebrae is investigated.

Figure 4.12 displays cranial, caudal and lateral views of 3D renders of selected vertebrae from the re-articulated skeleton. This image clearly highlights the remarkable changes of overall morphology from the cervical vertebrae through to the last lumbar vertebra, from both craniocaudal and lateral views.

The first thoracic vertebra have a similar morphology to the last cervical vertebra; with a large foramen, low volume and small processes. The thoracic vertebrae change dramatically from T1 - T14. T3 has a particularly prominent spinous process which reduces in height and ultimately splits by T14. T9 has enlarged transverse processes, which is where the first visible tubercles on the caudal side are seen. Superior and inferior processes are also far more prevalent by this stage. By T14, the vertebrae are very similar in appearance to the lumbar vertebrae, and almost rectangular in shape. The lateral edges of the front lumbar 


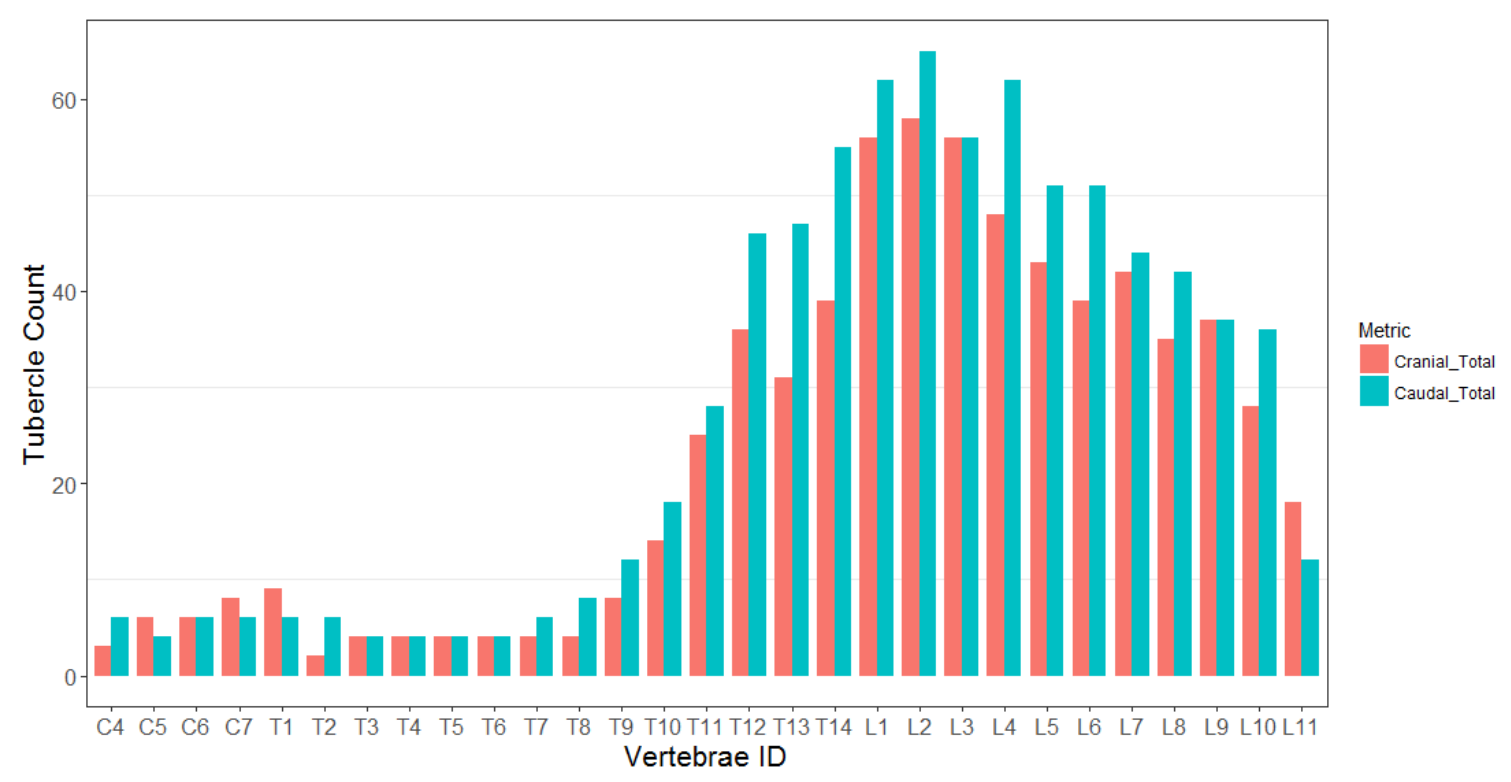

Figure 4.9: Tubercle count for each vertebra. Colour represents tubercles on the cranial or caudal faces of the vertebra.

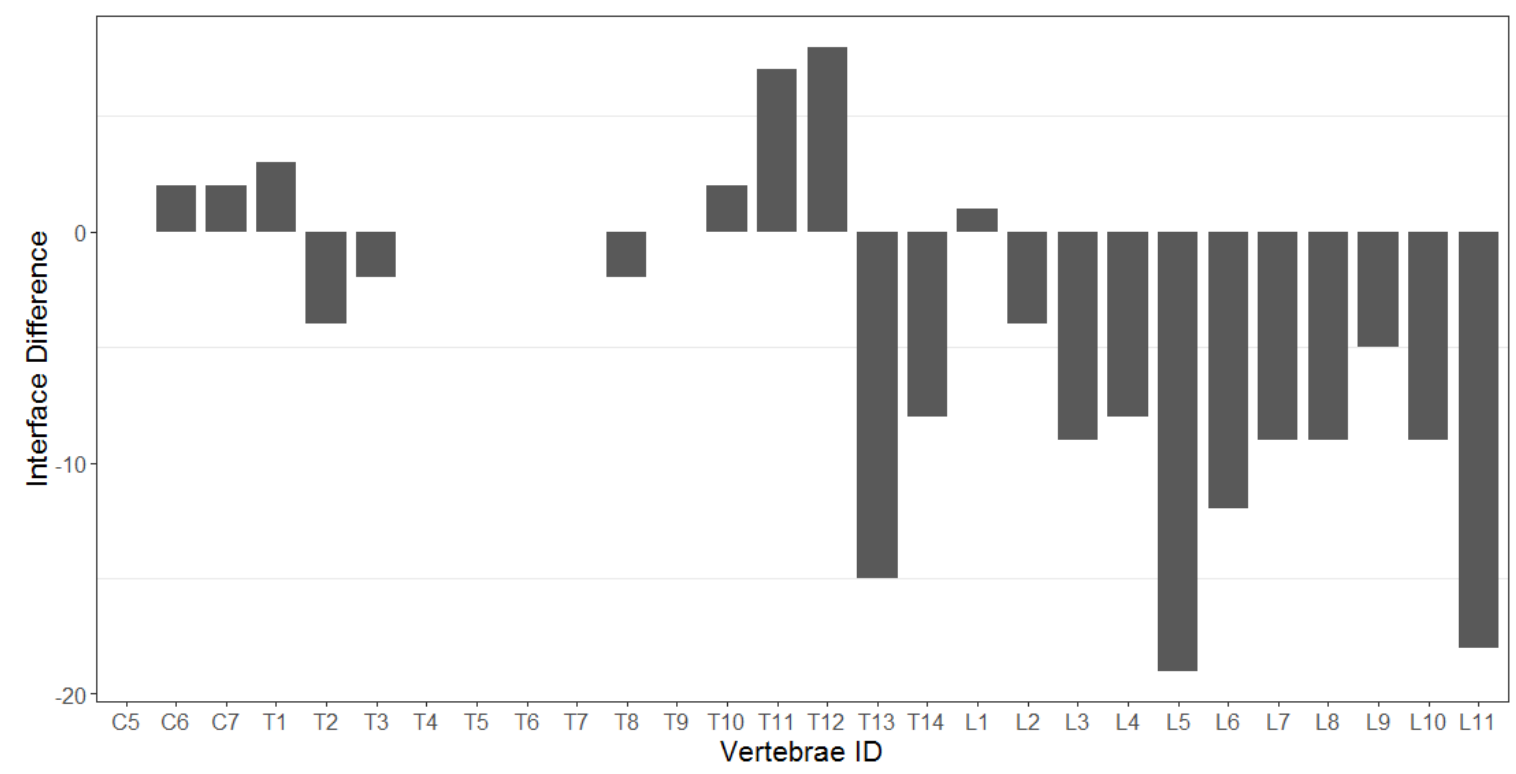

Figure 4.10: Tubercle count differences between the vertebra cranial face and the preceding vertebra caudal face. 


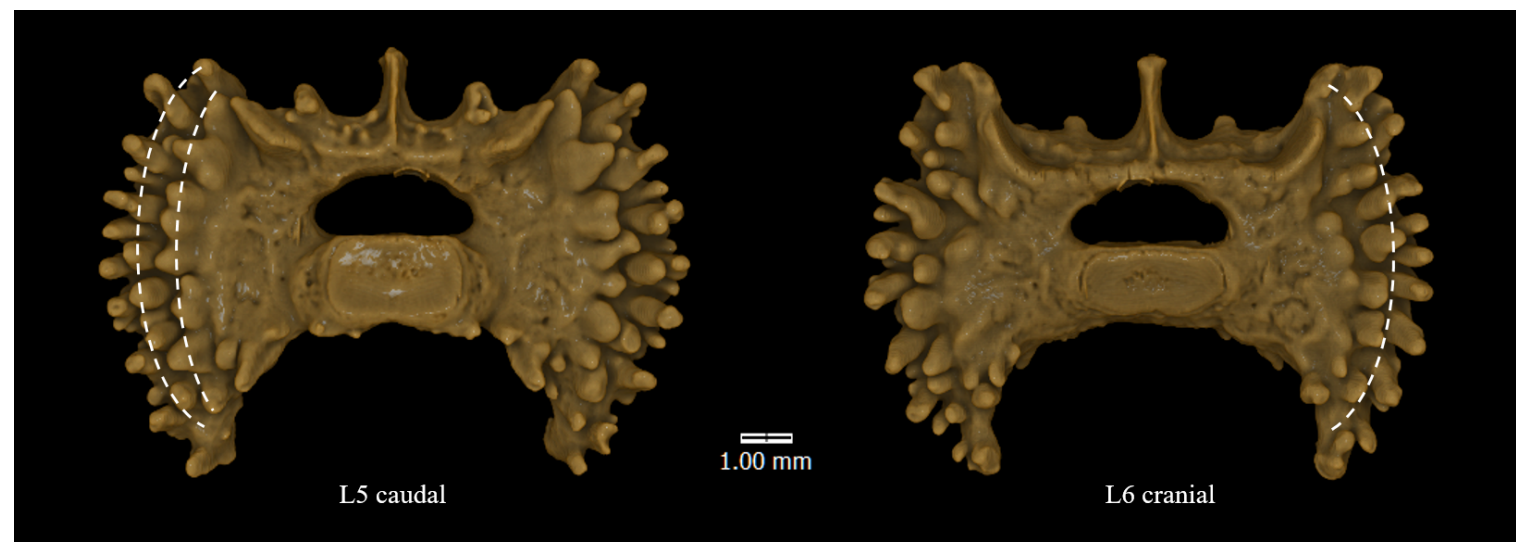

Figure 4.11: 3D renders of adjacent vertebrae L5 (caudal face) and L6 (cranial face). The tubercles indicated by the dashed line over L6 fit between the two sets of tubercles indicated by dashed lines over L5.

vertebrae become more curved, and the ventrolateral part is more pronounced. The spinous, superior and inferior processes are prominent. L9 and L11 at the rear of the lumbar region reduce in size and tubercle count, and the transverse processes are reduced and lose the curved shape of the front lumbar vertebrae.

A benefit of using X-ray $\mu \mathrm{CT}$ for the analysis of the hero shrew's vertebrae is that the internal structure is accurately captured. Using histological slides provided by Dr. Cullinane, the trabeculae structure can be correlated with the acquired X-ray $\mu \mathrm{CT}$ data, as shown in Figure 4.13. Although the exact vertebrae in the histological slides are unknown, it is clear that they are from the mid-thoracic to lumbar regions based on their morphology. The volume measurements used in this analysis take into consideration this internal architecture and therefore can be used as an approximate measure.

The internal structures of vertebrae from thoracic and lumbar regions are shown in Figure 4.14. It can be seen that the internal structure varies throughout a vertebra, with regions of both cancellous and cortical bone. The tubercles are composed of cortical bone for increased stiffness and strength, whereas the dorsolateral and ventrolateral regions, as well as the spinal and transverse processes are typically made up of cancellous bone. Cancellous bone is known to help reduce weight and aid energy absorption.

\subsubsection{Vertebrae sample comparison}

Whilst incomplete, it was possible to analyse regions of the samples acquired from Dr. Cullinane for comparison with the re-articulated skeleton. Vertebrae T1 - T14 were complete in sample US_sample2 and T1 - L2 in sample US_sample3. Data for these samples are given in Tables 4.3 and 4.4 respectively. Unfortunately, US_sample1 was too damaged to include in any comparative work.

Figure 4.15 displays, where available, the volumes of vertebrae T1 - L2 across the three 


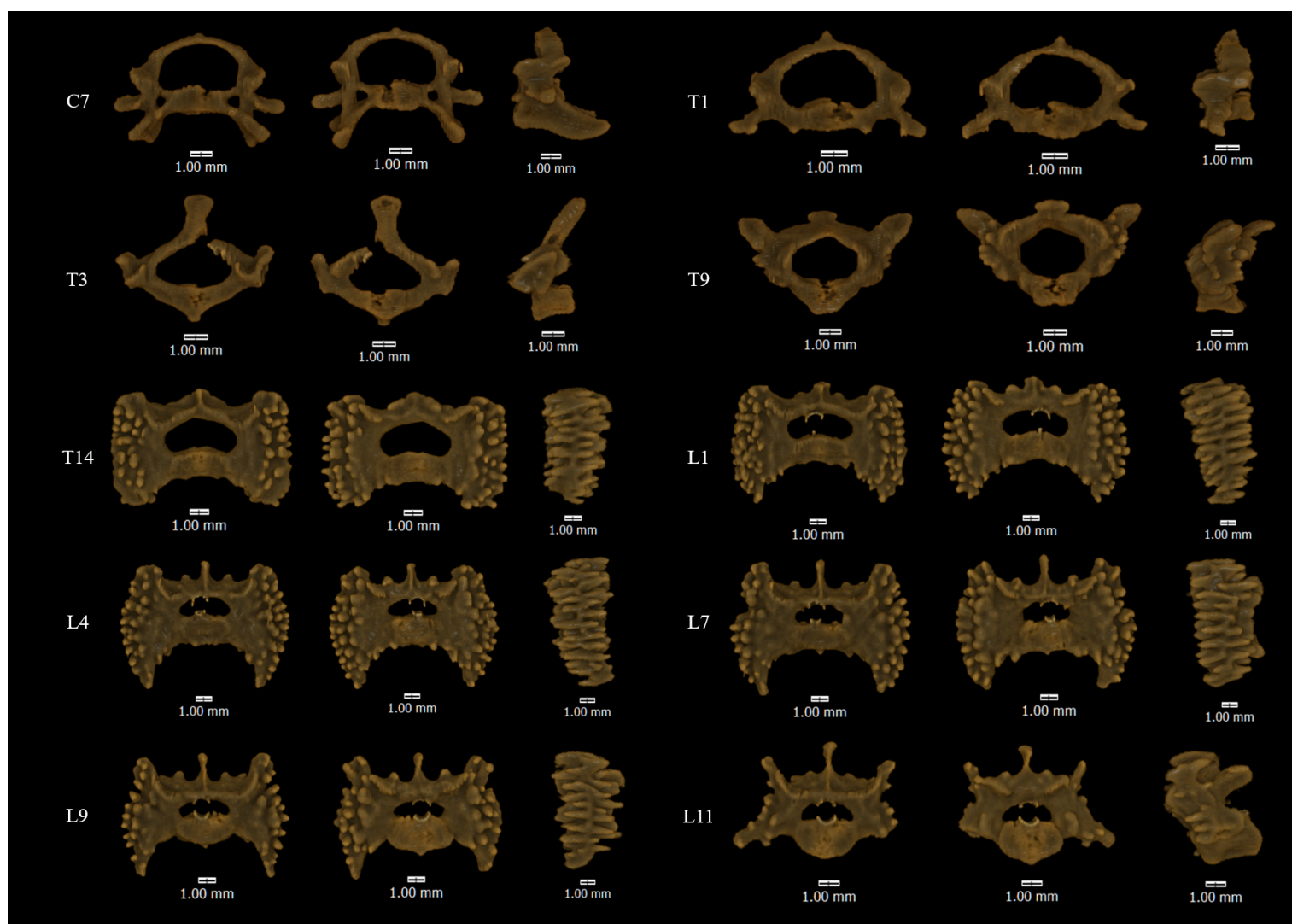

Figure 4.12: 3D renders of vertebrae from the re-articulated skeleton specimen. Views in the cranial, caudal and lateral directions respectively.

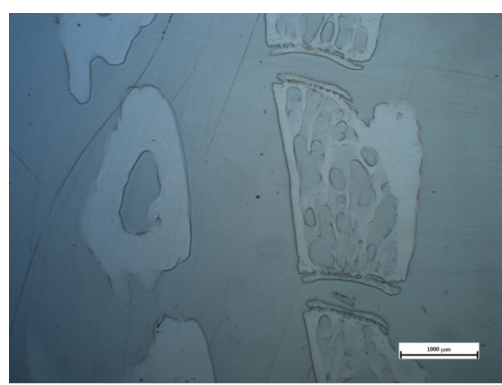

(a)

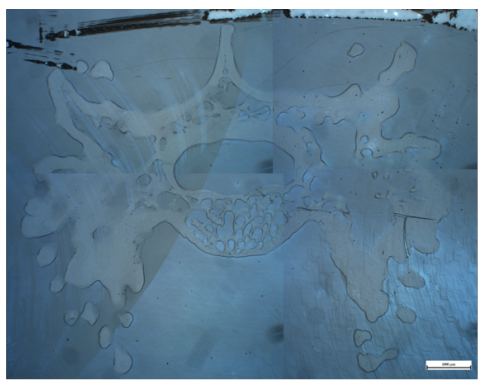

(b)

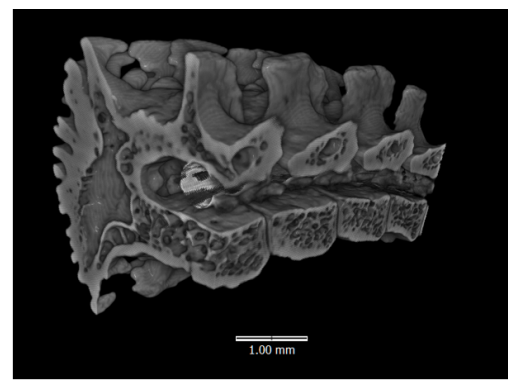

(c)

Figure 4.13: Internal structure of the hero shrew vertebrae. (a-b) composite optical images of histological slides provided by Dr. Cullinane showing cross-sections of vertebrae, (c) 3D render of re-articulated skeleton showing internal structure. 


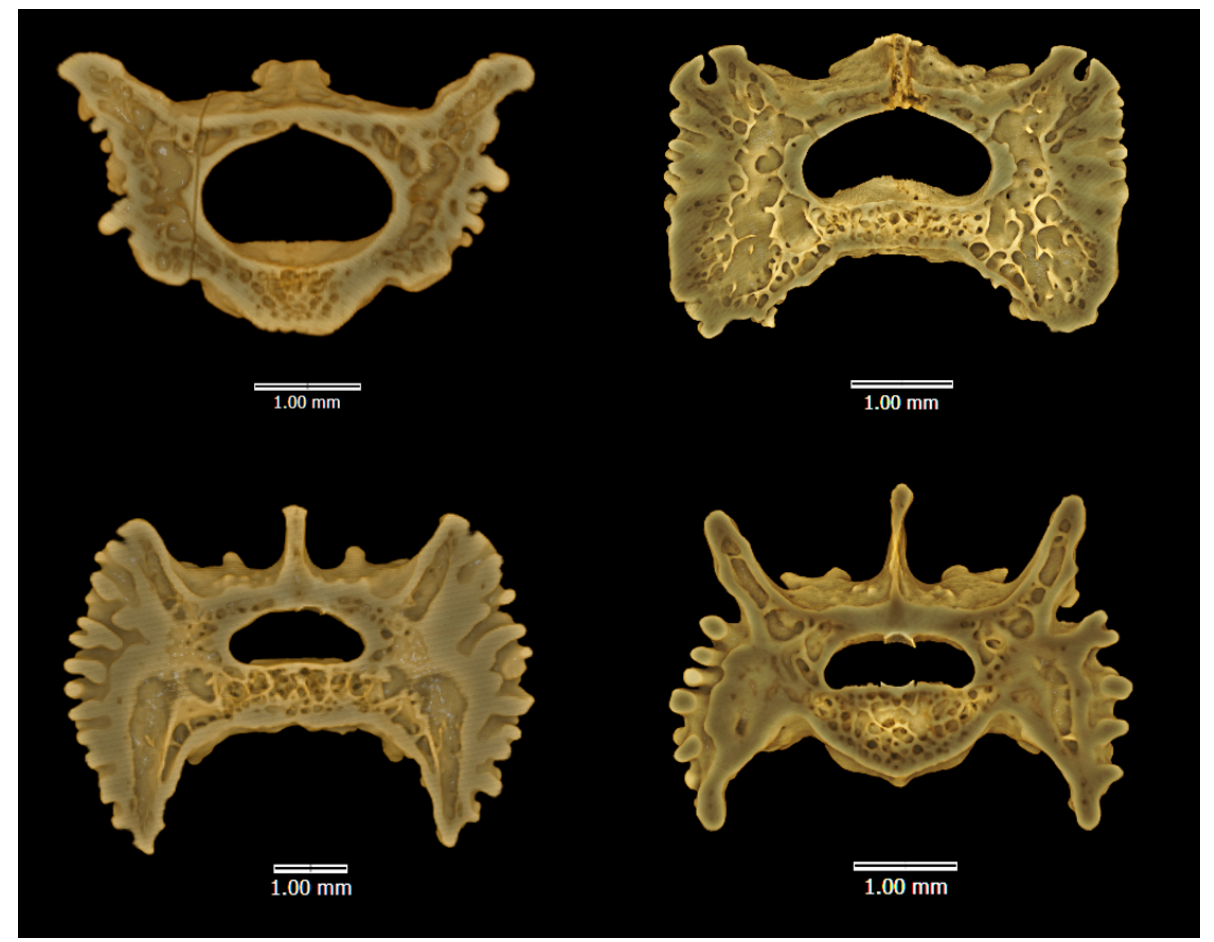

Figure 4.14: 3D renders of vertebrae virtually cropped to reveal internal structures.

Table 4.3: Summary of vertebrae analysis from US_sample2.

Vertebrae Volume Surface Area Total tubercle Tubercle count Tubercle count

\begin{tabular}{lrrrrr} 
ID & $\left(\mathrm{mm}^{3}\right)$ & $\left(\mathrm{mm}^{2}\right)$ & count & (cranially) & (caudally) \\
\hline T1 & 10.52 & 103.44 & 13 & 7 & 6 \\
T2 & 11.09 & 102.93 & 12 & 4 & 6 \\
T3 & 10.99 & 122.02 & 12 & 6 & 6 \\
T4 & 9.96 & 111.07 & 16 & 10 & 6 \\
T5 & 10.68 & 112.03 & 21 & 15 & 6 \\
T6 & 10.54 & 120.24 & 19 & 11 & 8 \\
T7 & 11.29 & 120.09 & 19 & 5 & 14 \\
T8 & 14.54 & 139.98 & 32 & 11 & 21 \\
T9 & 18.09 & 173.79 & 57 & 17 & 40 \\
T10 & 21.11 & 216.72 & 78 & 36 & 59 \\
T11 & 28.95 & 272.66 & 106 & 47 & 77 \\
T12 & 39.04 & 364.45 & 133 & 56 & 75 \\
T13 & 55.44 & 429.61 & 137 & 62 & 71 \\
T14 & 61.13 & 460.77 & 135 & 64 & \\
\hline
\end{tabular}


Table 4.4: Summary of vertebrae analysis from US_sample3.

\begin{tabular}{lrrrrr}
$\begin{array}{l}\text { Vertebrae } \\
\text { ID }\end{array}$ & $\begin{array}{r}\text { Volume } \\
\left(\mathrm{mm}^{3}\right)\end{array}$ & $\begin{array}{r}\text { Surface Area } \\
\left(\mathrm{mm}^{2}\right)\end{array}$ & $\begin{array}{r}\text { Total tubercle } \\
\text { count }\end{array}$ & $\begin{array}{r}\text { Tubercle count } \\
\text { (cranially) }\end{array}$ & $\begin{array}{r}\text { Tubercle count } \\
\text { (caudally) }\end{array}$ \\
\hline T1 & 8.42 & 76.50 & 13 & 6 & 7 \\
T2 & 8.48 & 75.76 & 8 & 2 & 6 \\
T3 & 9.47 & 87.10 & 11 & 5 & 6 \\
T4 & 8.53 & 78.94 & 10 & 4 & 6 \\
T5 & 8.80 & 84.39 & 10 & 4 & 6 \\
T6 & 8.62 & 89.24 & 8 & 4 & 4 \\
T7 & 9.63 & 93.65 & 13 & 4 & 9 \\
T8 & 11.31 & 109.38 & 23 & 6 & 17 \\
T9 & 15.68 & 135.14 & 45 & 18 & 27 \\
T10 & 19.70 & 168.21 & 68 & 33 & 35 \\
T11 & 26.61 & 216.09 & 94 & 43 & 51 \\
T12 & 32.39 & 259.94 & 93 & 46 & 57 \\
T13 & 38.29 & 292.29 & 95 & 45 & 61 \\
T14 & 45.38 & 319.84 & 121 & 60 & 69 \\
L1 & 57.24 & 377.09 & 125 & 56 & 64 \\
L2 & 71.83 & 434.05 & 127 & 63 & \\
\hline
\end{tabular}

samples. All three samples follow the same trend, however there is some difference in the vertebrae volumes across the samples. US_sample2 consistently has the largest vertebrae volumes. Since there is no available information of the background of the specimens, no conclusions can be drawn about the volume differences with respect to age, sex or habitat, for example.

Figure 4.16 displays the surface area of vertebrae T1 - L2 across the three samples. Again, all three samples show the same trend. US_sample2 consistently has the largest surface area of each vertebrae and the re-articulated skeleton consistently has the smallest. $\mathrm{T} 8$ is the first vertebra to start showing increases in both volume and surface area on all three samples.

Figure 4.17 displays the tubercle counts of common vertebrae across the three samples. There is significant variation in the counts from some of the vertebrae. For example, across vertebrae T8 - T11, US_sample2 has over twice as many tubercles on equivalent vertebrae as the re-articulated skeleton. US_sample3 shows counts somewhere between the other samples throughout T8 - T14, however counts for L1 and L2 between the re-articulated skeleton and US_sample3 are more comparable.

Figure 4.18 shows cranial views of vertebrae T3, T10 and T13 from the three samples. US_sample2, and to some extent US_sample3, shows more prominent vertebral features than the re-articulated skeleton. For example, the spinous process is larger, and the number of tubercles seen on T10 and T13 is significantly greater, which supports the findings in Figure 4.17. It is worth noting that the overall shape of the vertebrae remains consistent across all 


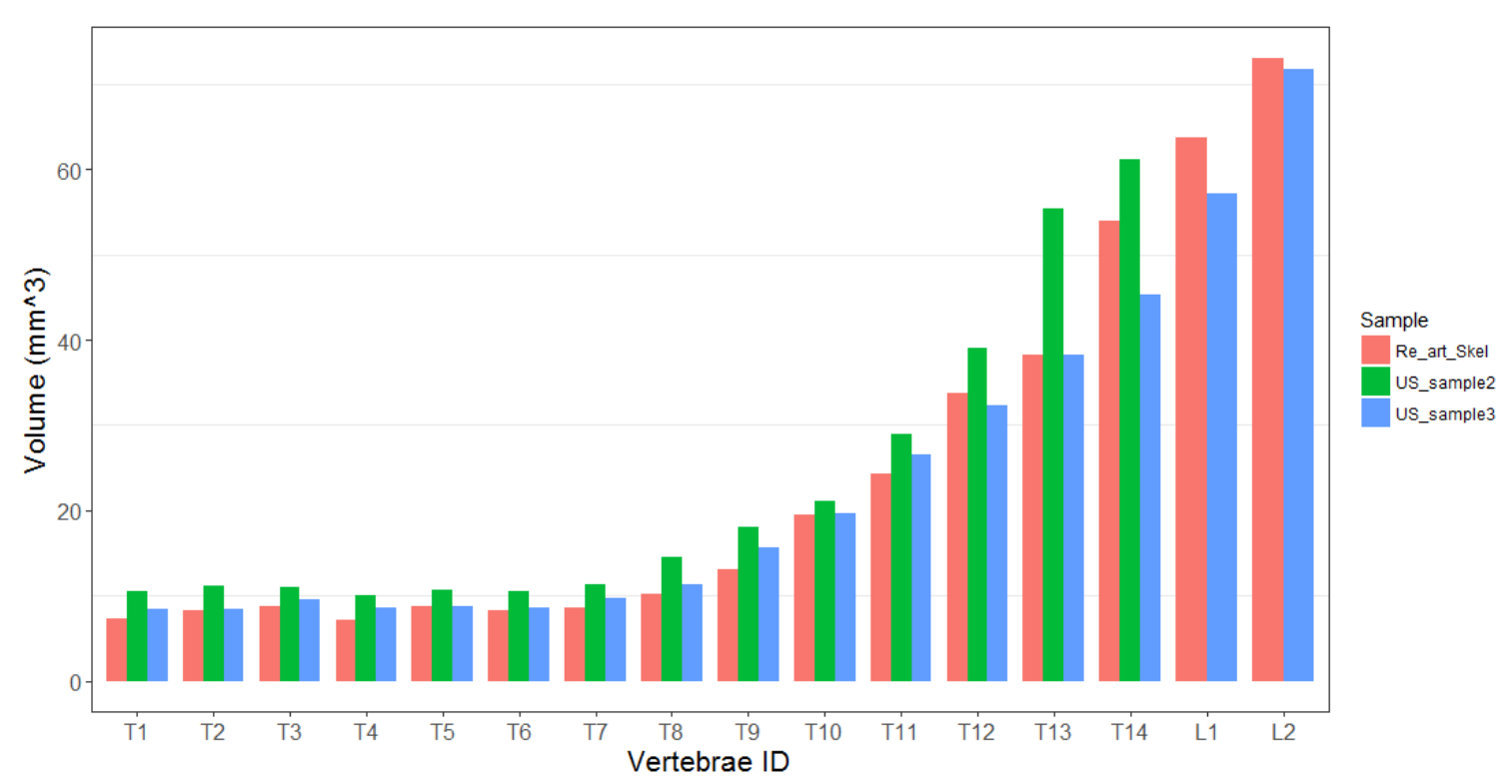

Figure 4.15: Vertebra volume $\left(\mathrm{mm}^{3}\right)$ of common vertebrae on three samples.

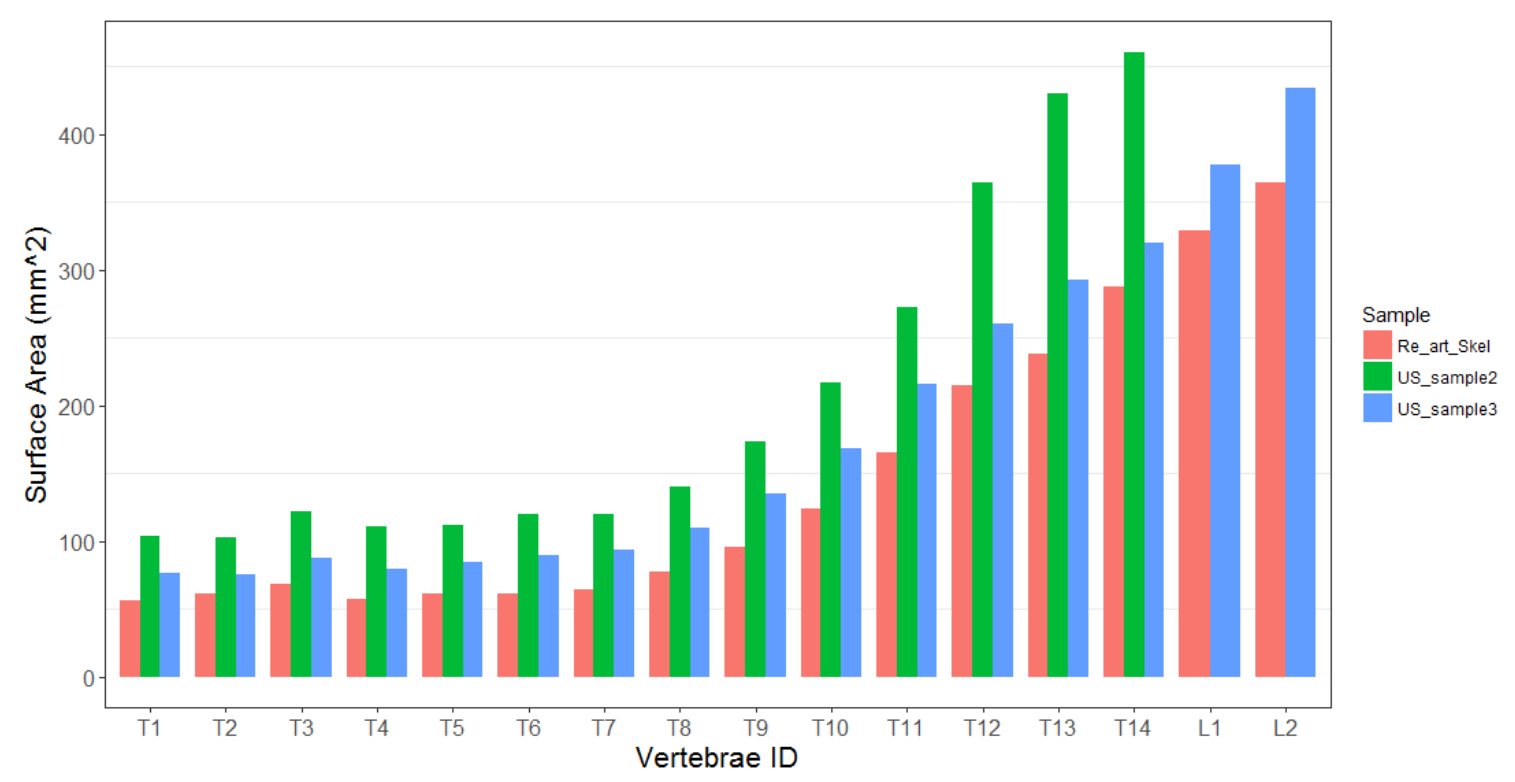

Figure 4.16: Vertebra surface area $\left(\mathrm{mm}^{2}\right)$ of common vertebrae on three samples. 
three samples.

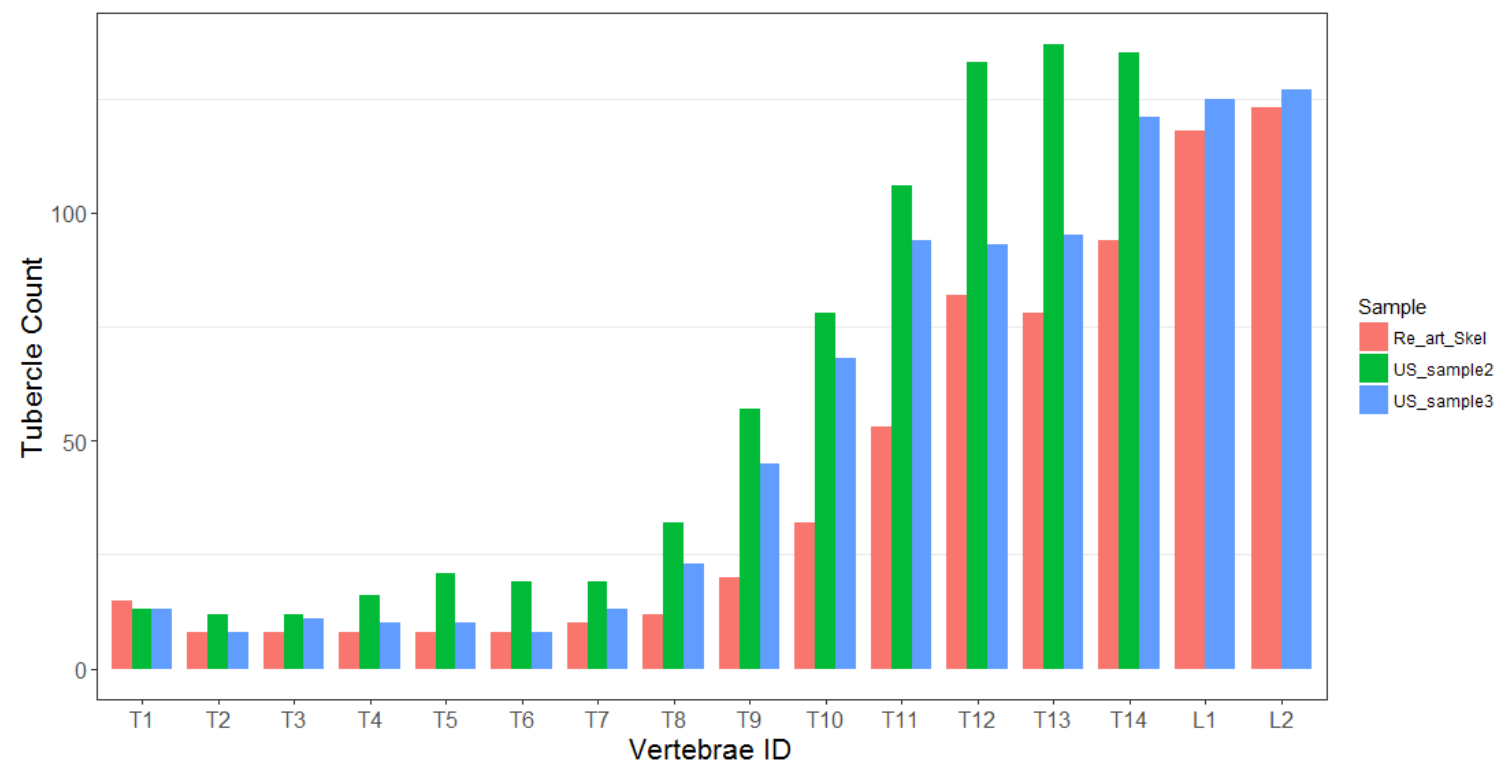

Figure 4.17: Tubercle counts of common vertebrae on three samples.

The scatterplots displayed in Figure 4.19 show the relationships between volume, surface area and tubercle count. It is clear that there is strong positive correlation between all three quantities. Trend lines are fairly sample specific when volume is considered against surface area or tubercle count. However the strongest relationship is between surface area and tubercle count, where all three samples follow the same trend, indicating a clear link between these two quantities.

In the previous section, the difference in tubercle counts at the interface of adjacent vertebrae was illustrated for the re-articulated skeleton, with the caudal thoracic and lumber vertebrae being of particular interest. Figure 4.20 compares these differences to vertebrae T8 - L2 of US_sample2 and US_sample3. Whilst the patterns seen on the re-articulated skeleton are not exactly replicated in the other two samples (T10, T12 and T14 show contrasting interface differences, for instance), there are clear similarities. At some stage between $\mathrm{T} 9$ and $\mathrm{T} 12$, each sample shows at least one interface where the overriding trend of caudal faces having more tubercles than neighbouring cranial faces (negative interface differences) is reversed. It is likely not a coincidence this reversal in trends occurs where the spine notably bends. Allen described the bend as a strong convex curve behind the shoulder [169], and can be seen in Figures 4.1, 4.3 and 4.4. Unfortunately, US_sample2 and US_sample3 do not include the majority of the lumbar region, and so patterns seen in the re-articulated skeleton cannot be compared and validated. 


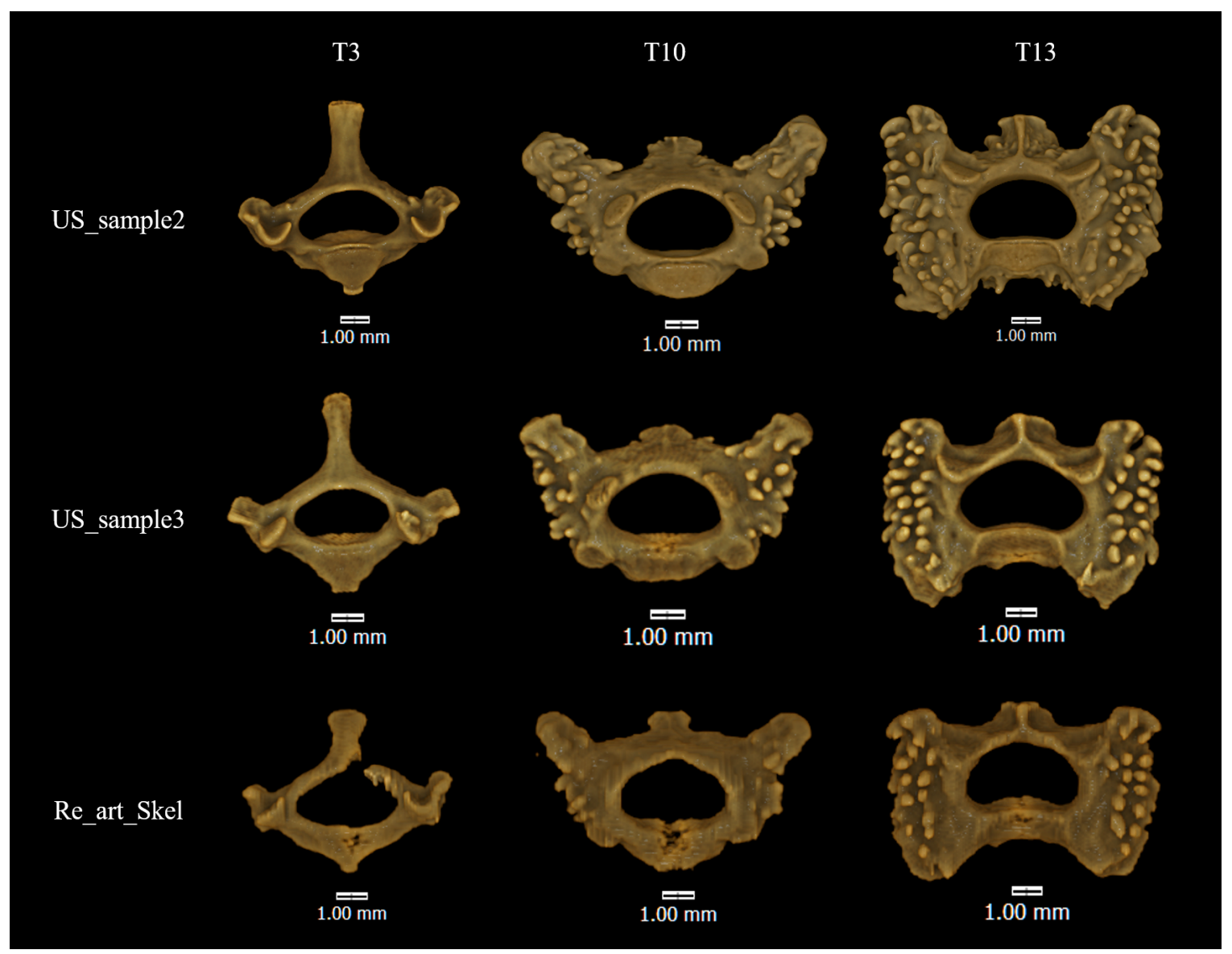

Figure 4.18: 3D renders of vertebrae from re-articulated skeleton, US_sample2 and US_sample3.
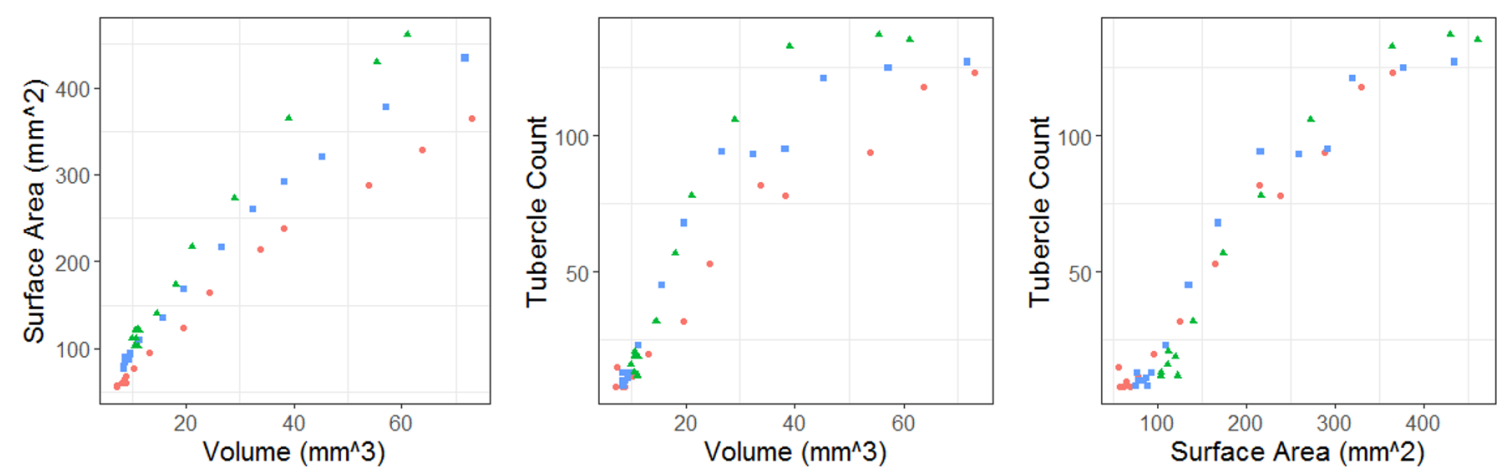

Figure 4.19: Scatterplots of volume vs surface area, volume vs tubercle count and surface area vs tubercle count, for vertebrae T1 - L2 of re-articulated skeleton (red), US_sample2 (green) and US_sample3 (blue). 


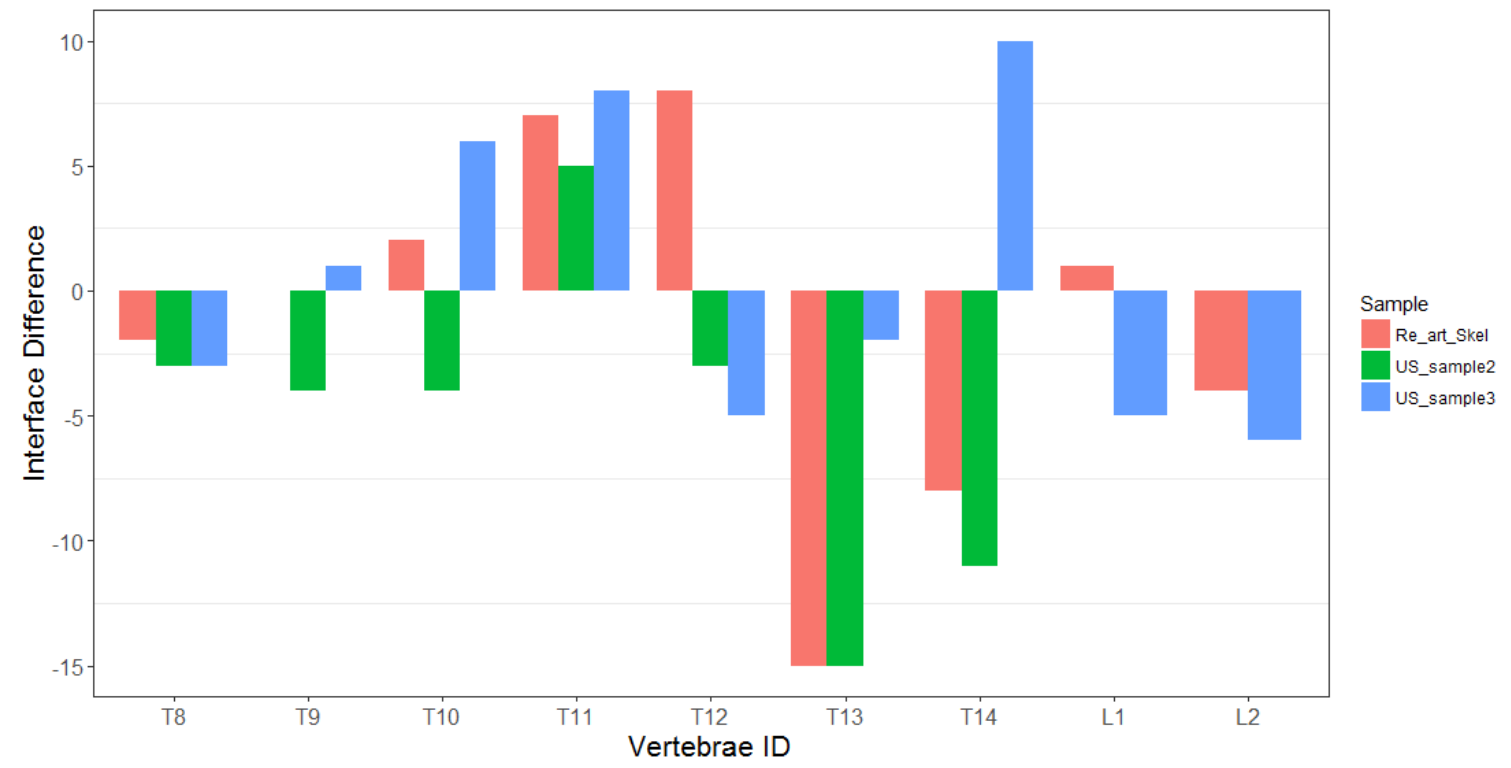

Figure 4.20: T8 - L2 tubercle count differences between the vertebra cranial face and the preceding vertebra caudal face.

\subsubsection{Vertebra interaction}

Given the unique structure of the hero shrew vertebrae, it is important to consider the effect of this unique morphology on the range of movement to help understand the impact vertebrae morphology has on it. L4, L5 and L6 are examined since they have the largest volumes and surface areas, and high tubercle counts. Torsional displacement has been assessed previously with the vertebrae under axial tension and compression [173]. However, no analysis of lateral flexion, flexion and extension has previously been conducted.

3D prints of L4 - L6 were manipulated to show their maximum displacement in relation to one another. Displacement was applied in the lateral flexion, extension and flexion directions, and the resulting angle between adjacent vertebrae was measured. Figure 4.21 displays dorsal and lateral views of the vertebrae at their maximum displacements. Table 4.5 reports the corresponding angles measured. It is clear from the angles in the table, and some of the images (Figure 4.21, dorsal view - lateral flexion), that L6 has greatest all-round movement. Immediately by reviewing Table 4.2 and Figure 4.10 it can be seen that there are fewer total tubercles between L5 and L6 than L4 and L5, and the difference in count between the caudal face of L5 and the cranial face of L6 (12 tubercles) is less than that of L4 and L5 (19 tubercles). A combination of these remarks would suggest that the number of tubercles has an effect on the lateral movement of adjacent vertebrae. Upon further inspection of 3D data, it appears the centrum is more pronounced in the caudal direction, which creates space between the vertebrae, allowing a greater angle of movement in the lateral flexion directions. There is further evidence for this in the lateral view of the right lateral flexion of Figure 4.21. The tubercles of L5 and L6 are more interdigitated than 
those of L4 and L5.

In contrast with the differences in the range of movement in the lateral flexion directions, L4 and L6 show equivalent movement in both extension and flexion directions. This suggests that the amount of tubercles is not related to the amount of movement in these directions, for these vertebrae at least. Extension movement is instead primarily impacted by the superior and inferior processes on the dorsal part of the vertebrae, and tubercles on the dorsolateral region to a lesser extent. Tubercles on the ventrolateral part of the vertebrae seem to have the largest impact on movement in the flexion direction.

Table 4.5: Angles of rotation from 3D prints of vertebrae L4 and L6 relative to L5.

\begin{tabular}{lrr} 
Direction & Angle (L4) & Angle (L6) \\
\hline Lateral flexion (LHS) & $2^{\circ}$ & $8^{\circ}$ \\
Lateral flexion (RHS) & $2^{\circ}$ & $12^{\circ}$ \\
Extension & $6^{\circ}$ & $5^{\circ}$ \\
Flexion & $12^{\circ}$ & $10^{\circ}$ \\
\hline
\end{tabular}

For comparison, 2D radiographs of the hero shrew [171, 173] in Figure 4.22 show the spine in lateral flexion, extension and lumbar flexion. Due to the image resolution, the angles cannot be measured directly however, they appear to be consistent with findings using the 3D printed lumbar vertebrae.

\subsubsection{Discussion}

The work in this chapter has used X-ray $\mu \mathrm{CT}$ to show that there are clear patterns of volume, surface area and total tubercle counts across the vertebrae. All three measurements are positively correlated, however the tubercle counts directly correspond to the surface area of each vertebra rather than volume, which includes the vertebral internal structure. The highest measurements are recorded on the mid-lumbar vertebrae which are at the most dorsal part of the spine. Without a clear understanding of the specific constraints encountered by these lumbar vertebrae, reasons for these patterns are difficult to determine.

There is significant variability in vertebrae measurements between specimens, however there are consistent patterns in the measurements within each sample, as the scatterplots in Figure 4.19 show. Insight into these variations could be gained with the knowledge of specimen and environmental factors, i.e. age of shrew, sex and habitat.

Although the placement of tubercles on an individual vertebra initially looks random, rough patterns are apparent on caudal and cranial faces, and there is an incredibly precise placement and fit of tubercles between adjacent caudal and cranial faces. This tubercle alignment has been shown to minimise torsional displacement whilst under axial compression, as shown in Cullinane [173]. This unique morphology could provide the basis for a design where flexibility is required but stability is essential when under compressive load-bearing 


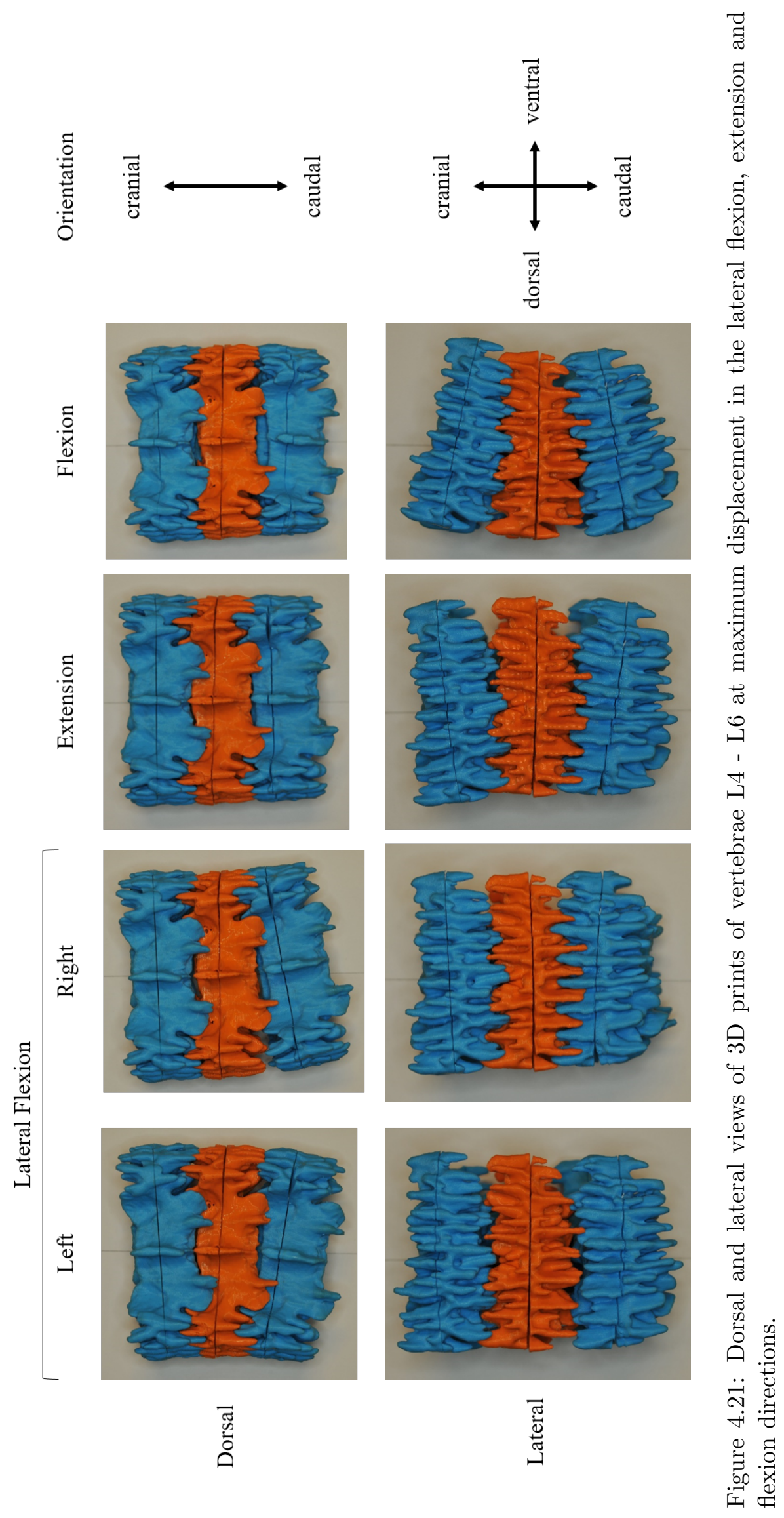




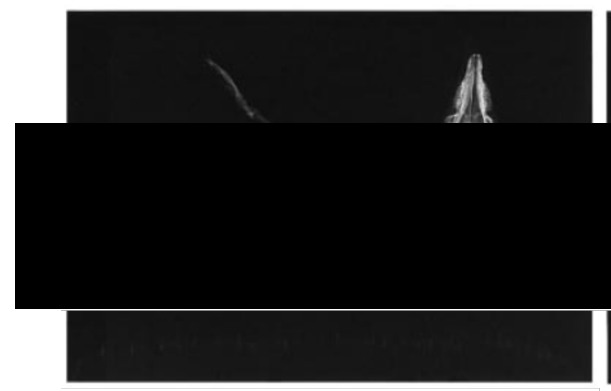

(a)

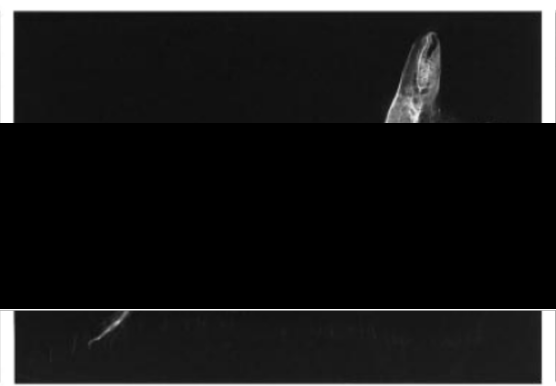

(b)

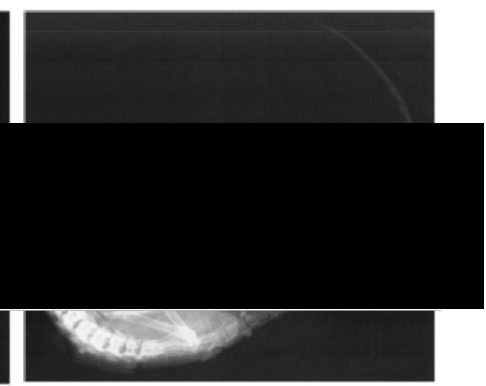

(c)

Figure 4.22: Dorsal and lateral radiographs of the skeleton of Scutisorex somereni. (a) spine in lateral flexion [171], (b) spine in extension [171], (c) spine in lumbar flexion [173].

scenarios.

A feature of the hero shrew spinal column is that it has 11 lumbar vertebrae instead of the usual 5 in mammals. This will increase flexibility due to an increased number of intervertebral joints, however the added constraints of the tubercles hinder this flexibility, as shown in section 4.3.3. A possible explanation for this contradictory situation is that the additional number of lumbar vertebrae give the hero shrew the flexibility it requires without compromising on the compressive strength and stability of the spine. Unfortunately, no clear reason of a requirement for this increased strength and stability has yet been determined, however one hypothesis is that the hero shrew uses its backbone as leverage when finding food under heavy or compressive objects [168].

3D printing provided a simple, efficient, low cost but useful initial insight into the range of movement of vertebrae and effects that features such as tubercle count and vertebra size have on this. Finite element modelling could be employed as a more sophisticated technique to analyse the full range of movement of the vertebral column. These models could consider the impact of soft tissue around the vertebral joints and potentially even the surrounding musculature. Simulations of different loading conditions could then be applied to these models which would provide further insight into the extreme modifications of the vertebral column.

Aside from its sister species (Thor's hero shrew), a vertebral column of this structure and morphology is considered unique amongst mammals. There are however similarities with suture structures found throughout nature. A suture structure consists of wavy or interdigitating interfaces with two phases: rigid suture teeth and a compliant interface layer [26]. Although this is one of the only bone-on-bone skeletal articulation cases recorded in mammals [173] there is a small muscle ( $\mathrm{m}$. intertubercularis) which exists between a tubercle and its articulating space on the neighbouring vertebra, which could act as the compliant interface layer. These suture structures appear in regions where there is a need to control intrinsic strength and flexibility: they allow a small amount of deformation until a critical load is reached at which point there is no further movement and this has been shown to be a 
functional trait of the hero shrew lumbar vertebrae. Examples of suture structures include the carapace of red-eared slider [175] and leatherback turtles [39], armadillo osteoderms [40] and boxfish scute junctions [111].

\subsection{Summary}

The use of X-ray $\mu \mathrm{CT}$ allowed for the quantification of vertebral morphology across the hero shrew spine, and both the internal and external features were accurately captured. Patterns between vertebrae volume, surface area and tubercle counts have been established and compared across 3 samples, these patterns were found to be consistent. The interface between neighbouring vertebrae was also considered and links were made with the range of movement established in previous research.

A simple method for quantifying the range of movement between highly modified vertebrae was demonstrated and links were made to tubercle counts between vertebrae. Suggestions for a more sophisticated analysis using finite element modelling were also given.

The hero shrew spinal morphology is of such interest because it is unique and its purpose remains somewhat of an enigma. Disregarding these points, it is clear that the spinal column exhibits exceptional mechanical properties through its flexural strength and resistance to torsion. Inspiration for applications where these characteristics are required, coupled with a degree of flexibility can be taken from this unique structure. 


\section{Chapter 5}

\section{Cuttlebone of Sepia officinalis}

\subsection{Introduction and previous work}

Cuttlebone, the internalised shell of Sepia officinalis (Linnaeus, 1758) found in all members of the order Sepiidae is a unique, chambered structure that acts both as a rigid structural component and the sophisticated buoyancy device of the cuttlefish [176]. It is composed of an upper dorsal shield and a lower lamellar matrix (Figure 5.1(a)). The lamellar matrix is made up of continuous chambers; separated by parallel lamellae (septa) and supported by a complex arrangement of pillars (as shown in Figure 5.1(b-c)). The lamellar matrix is composed of calcium carbonate $\left(\mathrm{CaCO}_{3}\right)$ in its aragonite polymorph with a mixture of organic material. The organic component is a $\beta$-chitin and protein complex $[177,178,179$, 180,181 ] that envelopes the aragonite matrix and contributes approximately $3-4.5 \mathrm{wt} . \%$ $[177,182]$ of the structure. The dorsal shield and septa have been identified as having both prismatic and lamellar structures (Figure 5.1(d-e)), however with different organisations. The dorsal shield is composed of three layers; the upper of prismatic tubercles, the middle of a lamellar structure and the inner of prismatic crystals [183]. The septa in the lamellar matrix consist of a lamellar structure on the upper side and a prismatic structure on the lower side, as found in the pillars [183, 184] (Figure 5.1(e)).

To efficiently perform the required functions of support and a lightweight buoyancy device, the cuttlebone must have an open structure that is resistant to the hydrostatic pressure of its environment whilst maintaining a constant volume. It has been shown that the cuttlebone has a porosity that exceeds $90 \%$ [177] whilst being able to withstand pressures of 2.4 MPa [185] encountered at habitation depths, which range from 100 to $500 \mathrm{~m}$ [186] [187] depending on the species. These exceptional mechanical properties of high compressive strength, high porosity and high permeability are extremely desirable for biomimetic and biomedical structural materials. Scaffold materials used in bone tissue engineering need to allow for the movement of nutrients and waste products, and also cell growth, whilst maintaining their structural integrity [188].

As the cuttlefish grows, the cuttlebone increases in proportion to the rest of its body. 


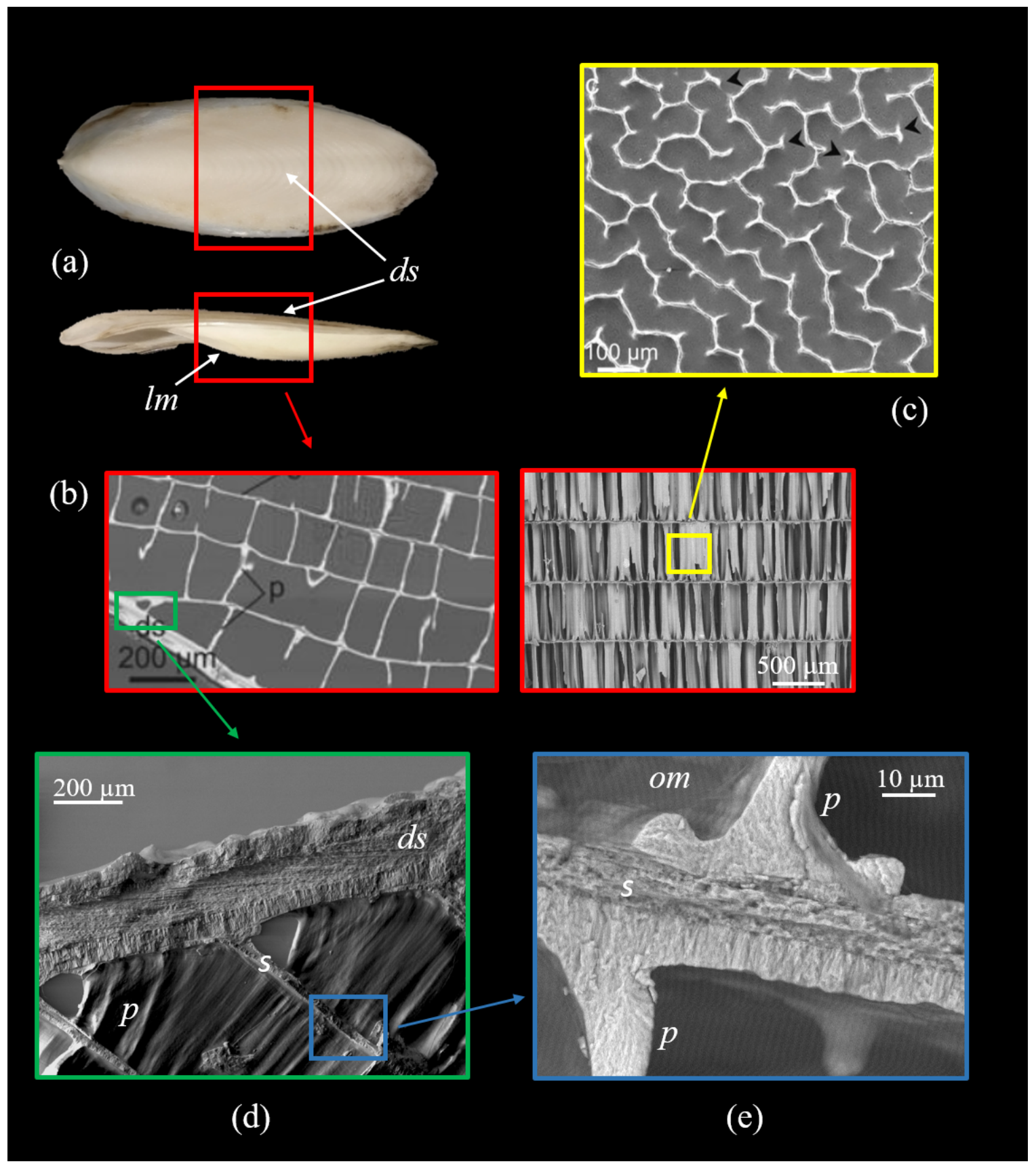

Figure 5.1: Schematic of a cuttlebone from Sepia officinalis: (a) macro images of a cuttlebone from dorsal and transverse views; (b) section through an embedded and polished specimen showing the dorsal shield and lamellar matrix (taken from [184]) and SEM image of a cross-section of lamellar matrix; (c) ventral view of the complex pillar morphology (taken from [184]); (d) SEM image of the dorsal shield and part of lamellar matrix; (e) SEM image of a fractured septum and pillars. $d s$ is the dorsal shield, $s$ is the septum and $p$ is a pillar, om is organic material, $l m$ is the lamellar matrix. 
Growth occurs in the anterior direction, and new lamellae are deposited ventrally with the extension of the dorsal shield at the anterior end. For the regulation of buoyancy, the older, posterior chambers fill with fluid via an osmotic mechanism in the siphuncular region [176] [185].

The structure of the cuttlebone was first described in 1893 by Appellöff [189], followed in more detail with the introduction of low-resolution SEM [177, 183, 190, 191]. These studies did not perform a full three-dimensional analysis, and were generally based on observations of visible surfaces, including when the samples were opened or fractured. In addition to the inorganic component, the role of the organic constituent has also been investigated [177, $181,182,184]$ and proposed to initiate chamber formation and organise the mineralisation of the inorganic structure, whilst also providing a toughening mechanism. It has been found that as well as the functional requirements mentioned above, the internal morphology of the cuttlebone was found to vary depending on species, age, environmental conditions and health of the individual $[177,191,192,193,194]$. This has led to research into how the microstructure of cuttlebone contributes to its mechanical performance [177, 191, 192].

Until recently, studies on the construction of the cuttlebone have been based on observations made by low-resolution SEM [190], which haven't considered the ultrastructure of the system and were therefore less than explicative. Checa et al. [184] propose a chamber formation model based on the physical processes of liquid crystallisation and viscous fingering. These mechanisms allow elements of the cuttlebone to be homologised with those of other coleoids and nautiloids.

To better understand features of the cuttlebone for reconstruction as a biomimetic material, examination of the chemical composition has been conducted [181] using Fourier transformed infrared analysis (FTIR), micro-Raman and particle induced X-ray emission (PIXE) mappings and X-ray diffraction (XRD). Based on the microstructural, mechanical and chemical characteristics of cuttlebone, novel applications have been investigated. Those proposed include the use of cuttlebone as a template for tissue scaffolding [195, 196, 197] and hydroxyapatite scaffolds [188, 198, 199, 200, 201, 202, 203, 204, 205]; filler for natural rubber [206], epoxy composites [207, 208, 209] and bone cements [210, 211]; and for creating superconducting materials [212]. For applications in bone tissue scaffolds and superconductor templates, issues with the final mechanical properties have been noted, potentially due to hydrothermal transformations or the variation in cuttlebone morphology. It is therefore necessary to examine and understand the structure-property relationships shown across the entire structure on the scale of whole devices.

Attempts to replicate the microstructure as a biomimetic material have involved the use of two-dimensional (2D) finite element-based homogenisation methods [213, 214]. It uses the morphology of a 2D Representative Volume Element (RVE), shown in Figure 5.2, and allows for the calculation of the effective mechanical properties of a periodic microstructure. This homogenisation of the structure does not take into account the complex morphology and variations found within individual samples of the cuttlebone (as noted by [183, 189, 
215]). Further research into the impact of morphological variations on the effective material properties using the homogenisation technique has shown to be of critical importance with respect to the effective bulk and shear moduli [216].

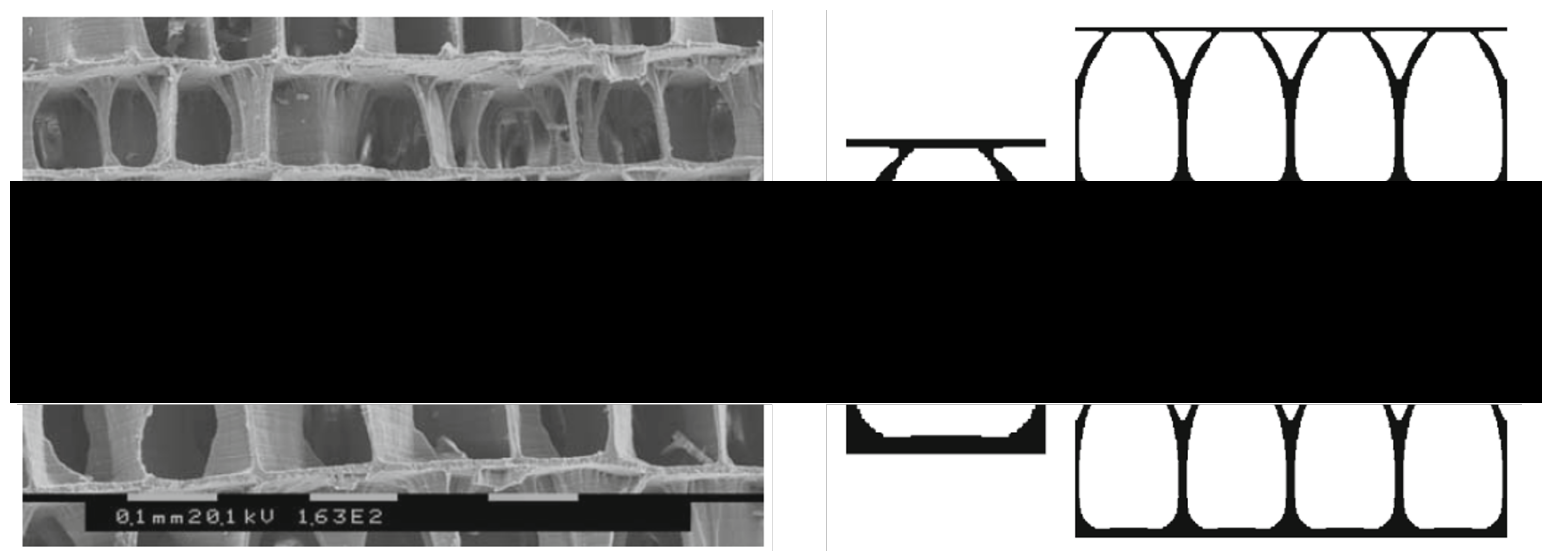

Figure 5.2: SEM image of transverse section of cuttlebone sample and unit cell model extracted from SEM images (scale bar $=0.1 \mathrm{~mm}$ ). Image modified from [214].

Having discussed previous studies of the complex structures within the cuttlebone, and identified the limitations of their methodologies, the objective of this study is to characterise the complex morphologies identified in the cuttlebone of Sepia officinalis in three-dimensions (3D). Utilising 3D imaging helps to minimise various challenges encountered using traditional microscopic methods. Cuttlebone has an anisotropic nature, and conventional 2D methods struggle to capture this. In addition, the field of view in a $2 \mathrm{D}$ setting is very limited, and preparation and mounting of samples is much more destructive. Unless already known, structural changes are not clearly evident and are sample and orientation dependent.

The chemical, structural and material properties of the hierarchical architecture and constituent components are investigated in this thesis using multi-scale analytical techniques, including X-ray microtomography ( $\mu \mathrm{CT}$ ), X-ray Photoelectron Spectroscopy (XPS), Electron Backscatter Diffraction (EBSD) and nanoindentation. This understanding of the structure-property relationships may have important implications for the use of cuttlebone as an engineering material and consideration of the microstructure for future biomimetic design applications.

In addition to the main objective, analysis of the cuttlebone microstructure, crystallography and chemistry will provide insights into the arrangement and growth of the structures within these marine organisms. This may have implications for further understanding of the efficient formation and design of biomineral structures, and also implications in reverse of the traditional bioinspiration pathway, where investigation helps inform understanding of form and function in nature. 


\subsection{Experimental methods}

There are three main sections to the study. The first section addresses the structural characterisation of the cuttlebone, the second section focuses on chemical differences and the third and final section considers mechanical properties.

\subsubsection{Specimens}

Fresh samples of cuttlebone were dissected from frozen juvenile specimens of Sepia officinalis, which were acquired commercially from www.baitbox.com and sourced around the UK coastline. Dissected samples were cleaned under running water to remove excess organic matter and stored in a freezer at $-20^{\circ} \mathrm{C}$ until use. These samples were then air-dried at room temperature for a minimum of 24 hours. Other dry samples of cuttlebone used for complementary analyses were collected from local beaches around the Gower Peninsula. Only samples with minimum damage were collected and these ranged in sizes from approximately $10 \mathrm{~cm}$ to $30 \mathrm{~cm}$. All experiments were performed under ambient conditions.

Figure 5.3 indicates the orientations used in the preparation of cuttlebones for analyses. Samples were cut along the longitudinal, transverse or dorsoventral axes to analyse features of interest. For each analysis, specific sample preparation is detailed in the relevant sections.

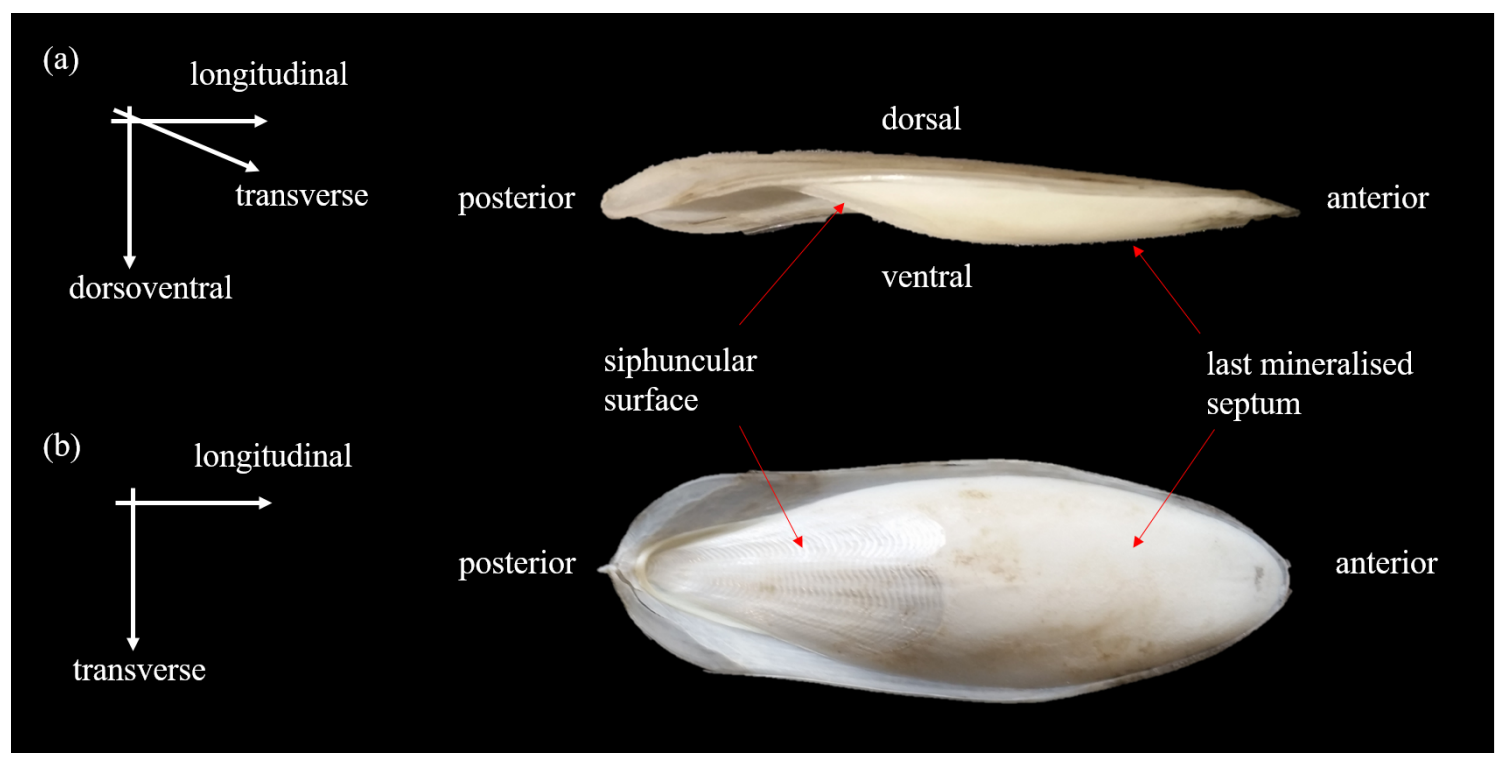

Figure 5.3: Representation of the principal axes for preparation of the cuttlebone, with regional descriptions: (a) transverse view; (b) ventral view, showing the siphuncular surface (area with ridged pattern) and the last mineralised septum (smooth surface). 


\subsubsection{Structural characterisation}

This section describes the methods used to characterise the structure of the cuttlebone: X-ray microtomography, scanning electron microscopy and electron backscatter diffraction.

\subsubsection{X-ray microtomography}

All tomographic data used in this study was acquired using the Nikon XT H 225 and Zeiss Xradia Versa 520 microcomputed tomography $(\mu \mathrm{CT})$ scanners, at the Advanced Imaging of Materials (AIM) facility at Swansea University. Samples of both freshly dissected and dried cuttlebones of a range of sizes were scanned.

Acquisition parameters for samples discussed in this study are provided in Table 5.1. Tomographic datasets were reconstructed in CT Pro and XMReconstructor for scans acquired on the Nikon XT H 225 (Nikon Metrology, Tring, UK) and Zeiss Xradia Versa 520 (Carl Zeiss X-ray Microscopy Inc., Pleasanton, CA) CT scanners respectively. 3D visualisation and manipulation of the data was achieved in VGStudio MAX 2.1 (Volume Graphics GmbH, Germany) and Drishti [217]. For large samples requiring multiple scans (as detailed in the final column of Table 5.1), these volumes were manually stitched in VGStudio in the $x, y$ and $z$ planes to create a complete dataset.

Volumetric datasets of cuttlebone were acquired to perform the quantification of the pillar morphology, time-lapse CT compression tests, and for general observations made about the morphology of the cuttlebone. Therefore where needed, discussion of X-ray CT is included in these sections.

\subsubsection{Quantitative analysis}

Quantitative analysis was carried out in three dimensions on morphological features of the cuttlebone using the processing programme ImageJ. Cores of cuttlebone were taken at intervals along the longitudinal and transverse axes, as shown in Figure 5.4. The cores were scanned and 3D datasets were acquired.

Over 80 scans from 22 different Sepia officinalis cuttlebone specimens were visualised. These specimens covered a range of sizes and ages. The features seen in the detailed example in this study are representative of the species and not just the individual.

The cuttlebone studied in this case measured around $11 \mathrm{~cm}$ so it can be said with confidence that it is a juvenile specimen. However, due to the known effects that environmental conditions have on the growth rates and lamellar deposition [194, 218, 219], the exact age of the specimen cannot be stated.

Seven core samples were taken from the cuttlebone in a dorsoventral direction to ensure each sample captured chambers from the dorsal shield to the ventral edge. Each sample core measured approximately $0.5 \mathrm{~cm}^{3}$ and a virtual core measuring $2 \mathrm{~mm}$ in diameter was captured from each sample core during the imaging process. Cores $1 \mathrm{~b}, 2 \mathrm{a}, 2 \mathrm{~b}$ and $3 \mathrm{c}$ are 


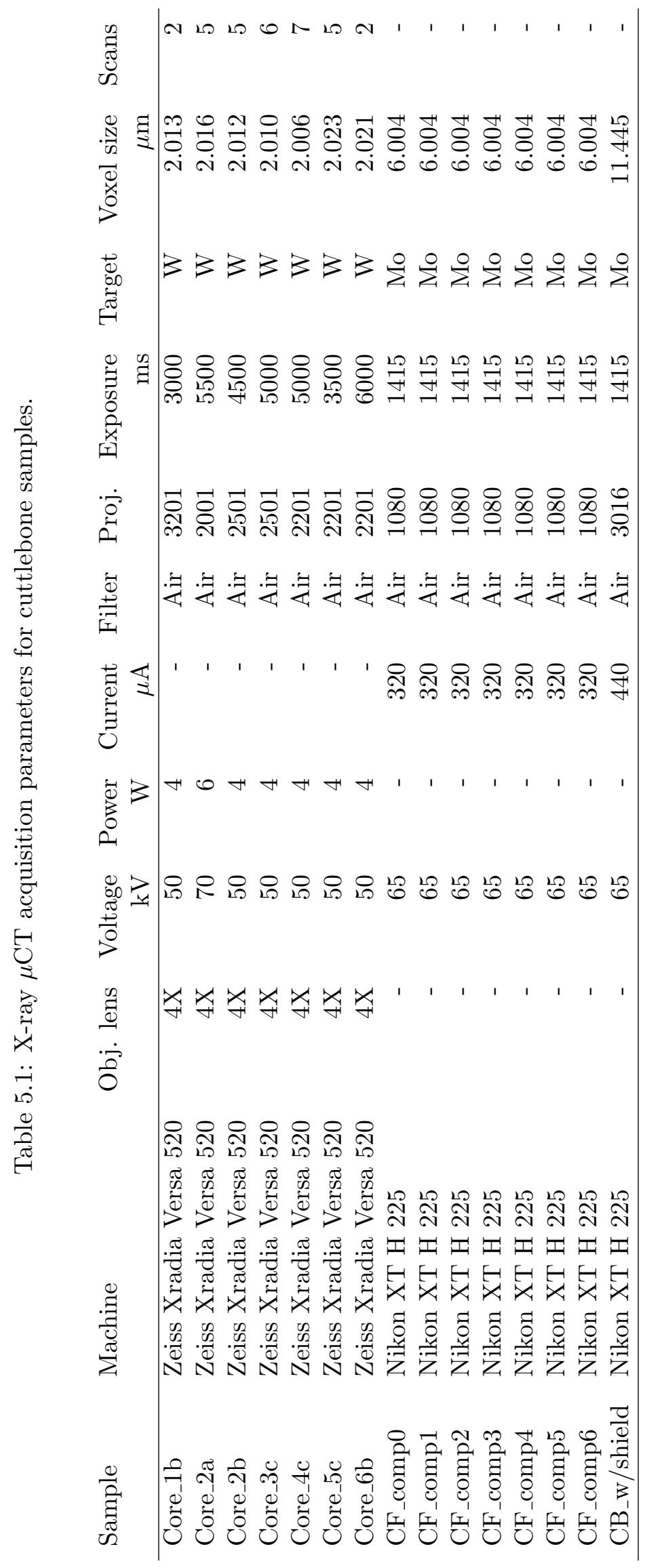




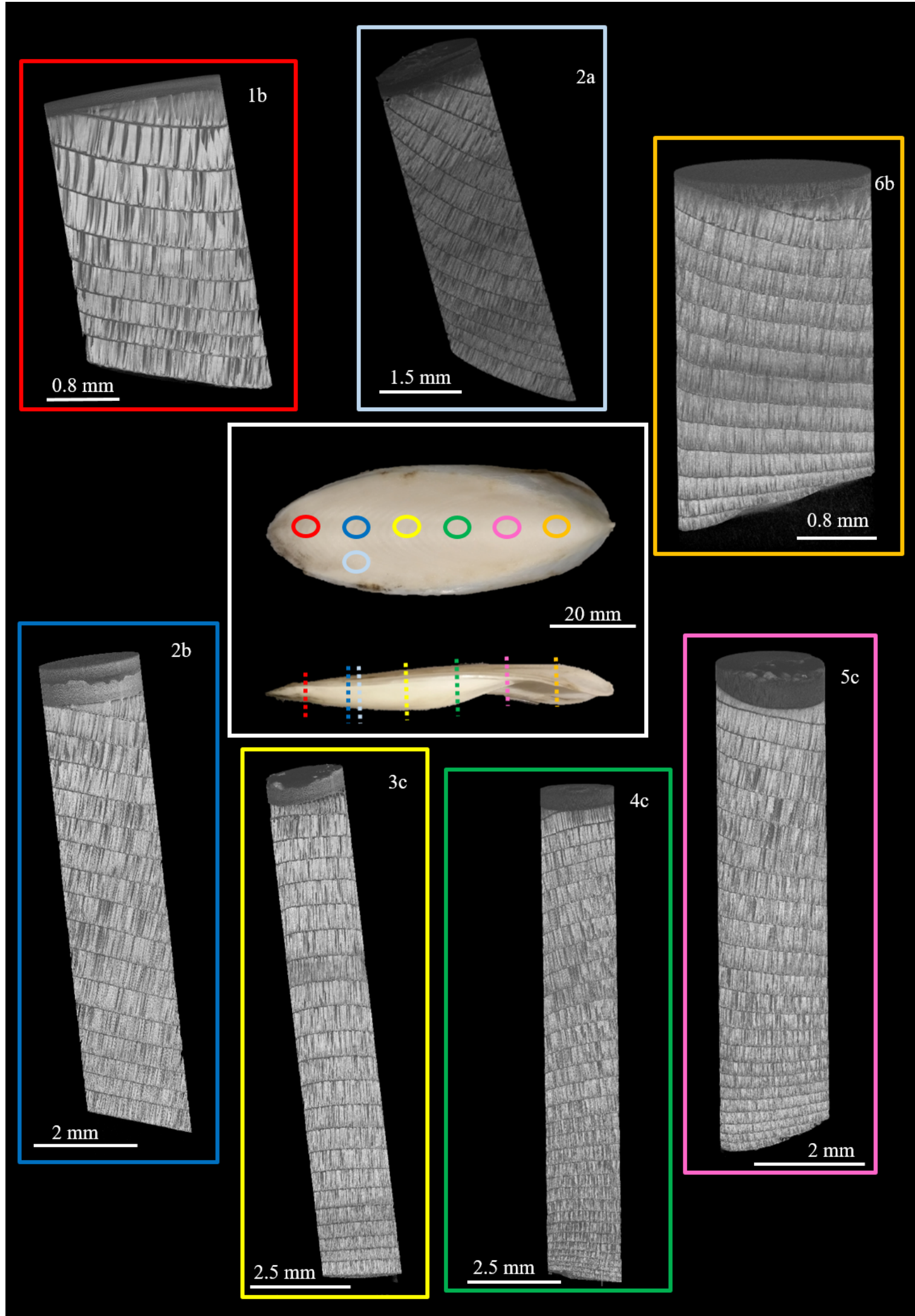

Figure 5.4: Schematic of cores used for analysis of morphological features within the cuttlebone. Cores are identified by the coloured boxes and paired with the coloured circles on the cuttlebone image. 
Table 5.2: Position of sample cores relative to the total length and width of cuttlebone.

\begin{tabular}{lrr} 
Core & $\begin{array}{r}\text { Longitudinal axis } \\
\text { (\% total length) }\end{array}$ & $\begin{array}{r}\text { Transverse axis } \\
\text { (\% total width) }\end{array}$ \\
\hline Core_1b & 8 & 50 \\
Core_2a & 25 & 15 \\
Core_2b & 25 & 50 \\
Core_3c & 42 & 50 \\
Core_4c & 59 & 50 \\
Core_5c & 75 & 50 \\
Core_6b & 92 & 50 \\
\hline
\end{tabular}

taken in the anterior area of the cuttlebone and the most ventral chamber is the most recently formed chamber. Cores $4 \mathrm{c}, 5 \mathrm{c}$ and $6 \mathrm{~b}$ are taken in the siphuncular zone.

In order to make comparisons with other specimens, the positions of the cores can be considered as a percentage of the cuttlebone longitudinal and transverse lengths. Figure 5.5 displays the orientation of the longitudinal and transverse length percentages, and the relative position of the cores used in this analysis are given in Table 5.2.

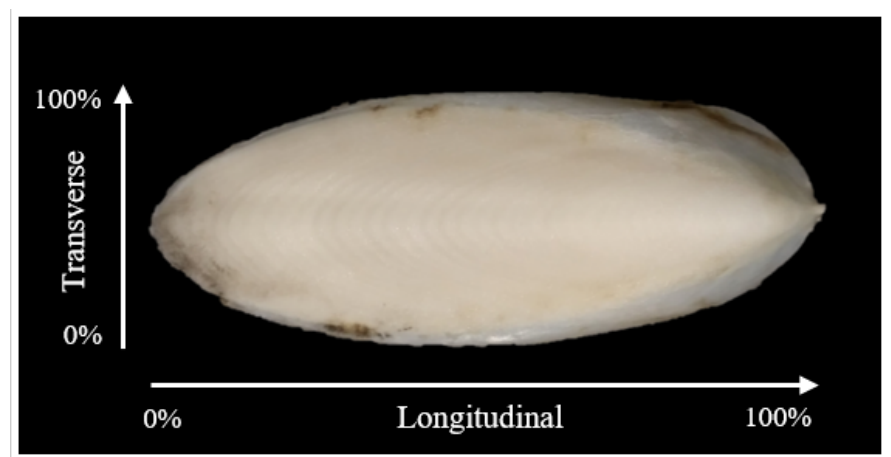

Figure 5.5: Longitudinal and transverse axes with percentages used to reference a position on the cuttlebone with respect to directional length. $50 \%$ on the transverse axis equates to the medial axis.

Tests involving various core sizes were conducted to optimise the trade-off between: maximising representative area to capture as many complete pillars as possible within a core sample; and minimising partial volume effects, which would lead to inaccurate pillar measurements.

As the examples in Figure 5.6 show, regions of similar pillar morphology are found both across larger areas than the cores used in this work, and also across multiple cuttlebones. Therefore, it was found that six cores taken along the medial axis was reasonable in order to accurately capture variations in pillar morphology along the length of the cuttlebone. Differences along the lateral edge were less obvious than the medial axis, therefore one core from the lateral edge was sufficient in this case to capture behaviour. 


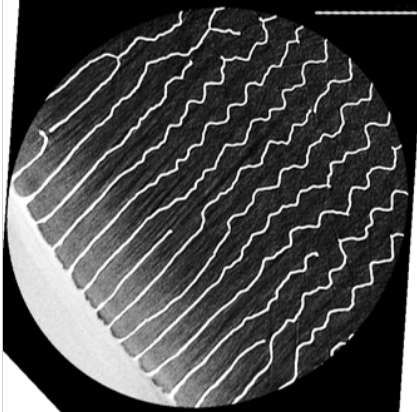

(a.1)

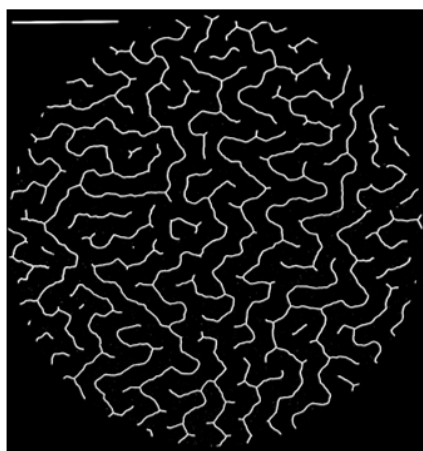

(b.1)

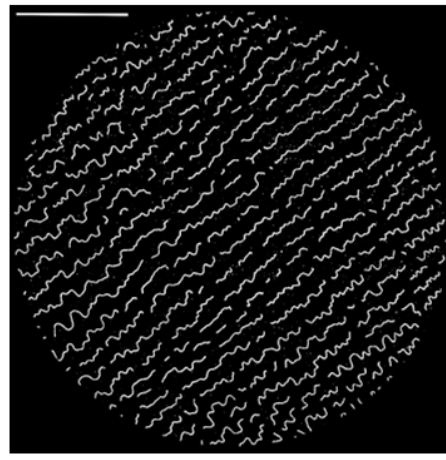

(c.1)

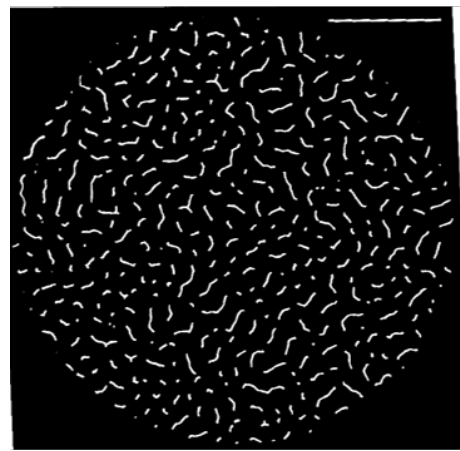

(d.1)

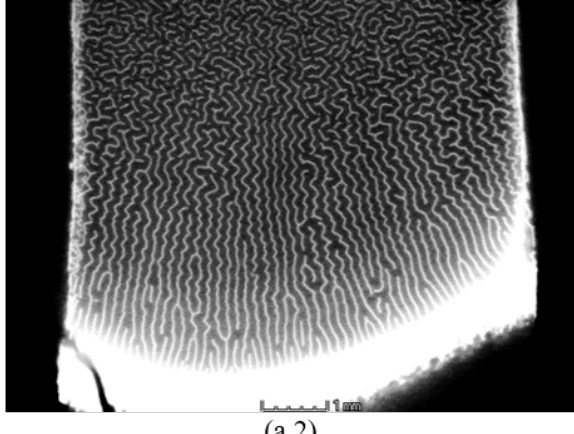

(a.2)

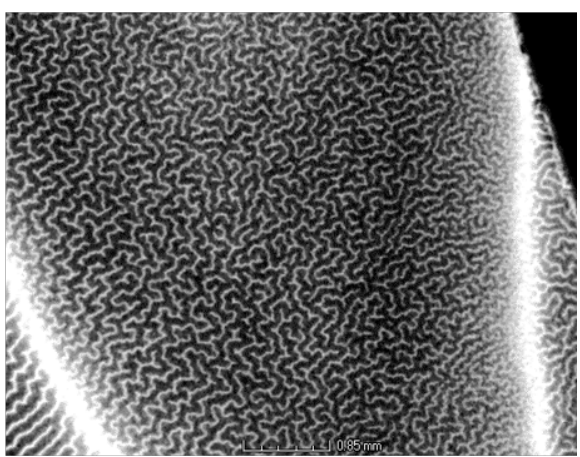

(b.2)

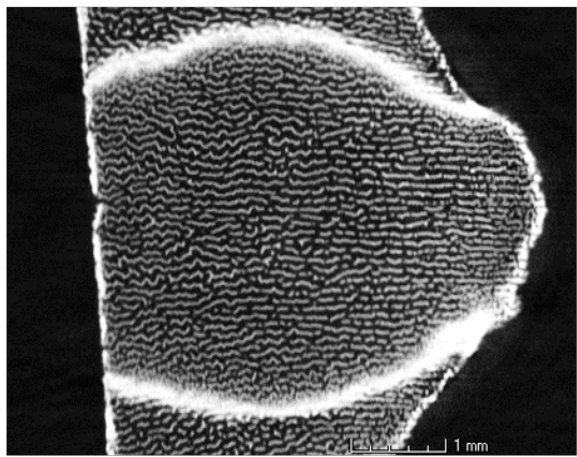

(c.2)

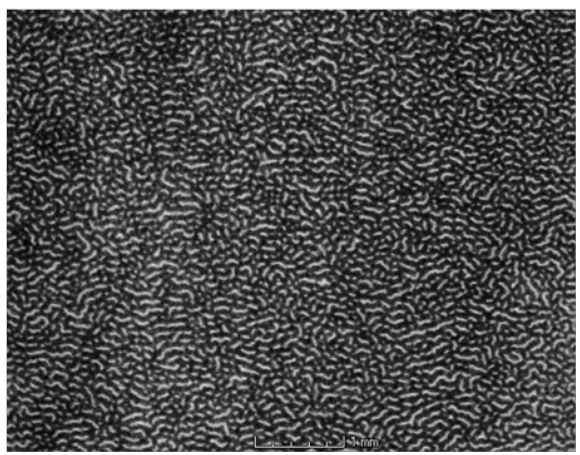

(d.2)

Figure 5.6: Comparison of different cuttlebone samples showing regions of similar pillar morphology with respect to longitudinal and transverse axes: (a) anterior region close to dorsal shield ( $5 \%$ longitudinal, $50 \%$ transverse); (b) centre of cuttlebone midway between dorsal and ventral edges $(50 \%, 50 \%)$; (c) posterior region towards ventral edge $(90 \%, 50 \%)$; (d) centre of cuttlebone towards ventral edge $(50 \%, 50 \%)$. Scale bar on all (.1) images = $0.5 \mathrm{~mm}$. 
For analysis of the pillars, 2D slices of individual chambers were exported from the 3D volumes of the cores. To account for the arcuate shape of the cuttlebone, each chamber had to be realigned so that the exported slices were parallel to the septa. This ensured the measurements of the pillars were taken at their cross-section. It is worth noting that the numbering of the chambers begins at the chamber closest to the dorsal shield, and so chamber 1 in one core may not necessarily be the same as chamber 1 in another core.

The slices of each chamber were exported at $0.004 \mathrm{~mm}$ spacing to give a detailed overview of any morphological variations within a chamber. The minimum distance between slices would be equal to the voxel resolution, being, in the case of this work, $0.002 \mathrm{~mm}$. No further information could be captured at any distance less than this value. Little additional information was captured at the minimum slice interval over the chosen spacing of 0.004 $\mathrm{mm}$, whilst producing twice the volume of data. A number of chambers in the ventral region, particularly at the posterior, had recorded heights of $<0.08 \mathrm{~mm}$, therefore a larger slice spacing would have resulted in very few slices capturing variation in pillars through the chamber. Figure 5.18 shows six slices from core $4 \mathrm{c}$ - chamber 10 with an effective spacing of $0.08 \mathrm{~mm}$ between them. There are visible differences in pillar morphology across the slices that are more effectively captured at $0.004 \mathrm{~mm}$ spacing.

A small sample of slices were removed from the extremes of the image stacks, which included noise due to the curved shape of the septa. The criteria for removing slices from analysis are discussed later in this section and in the results (section 5.3.1.1).

The image stacks of each chamber were imported into ImageJ, scaled, cropped and binarised. Figure 5.8 depicts this process. A number of thresholding methods were tested on the data, with the most consistent and accurate measurements being based on the $\mathrm{Li}$ method [220]. This thresholding method was applied to all images, with a minimum area set at $0.00009 \mathrm{~mm}^{2}$ to avoid capturing any noise. Measurements of the pillars were acquired using the Analyze Particles and BoneJ [221] plugins. Count, area, perimeter, Feret length, pillar thickness and spacing were among the measurements recorded as well as an estimation of tortuosity (equation (5.2.2.1)) and percentage of material-to-air. These measurements were consistent when compared with measurements captured using a SEM. In addition to this, an X-ray CT dataset was acquired using a much smaller isotropic voxel size of $0.7 \mu \mathrm{m}$, which further increased confidence in these results.

To validate pillar measurements, a series of measurements were taken using two extreme threshold levels to capture any variation. Five pillars from slice 40 of chamber 10 of core_4c were amongst those tested, these are shown in Figure 5.7. The threshold values used to binarise the image were: lower - 21-255; used in analysis - 41-255; and upper - 61-255. The results are shown in Table 5.3. It can be seen that the only measurement showing any real influence from threshold level is area, with roughly a $\pm 10 \%$ difference. This is understandable because pixels are progressively trimmed from pillars as the threshold increases, causing the pillar area to decrease. Perimeter and Feret length show very little influence. Therefore the threshold levels used throughout the analysis are deemed reasonable. 


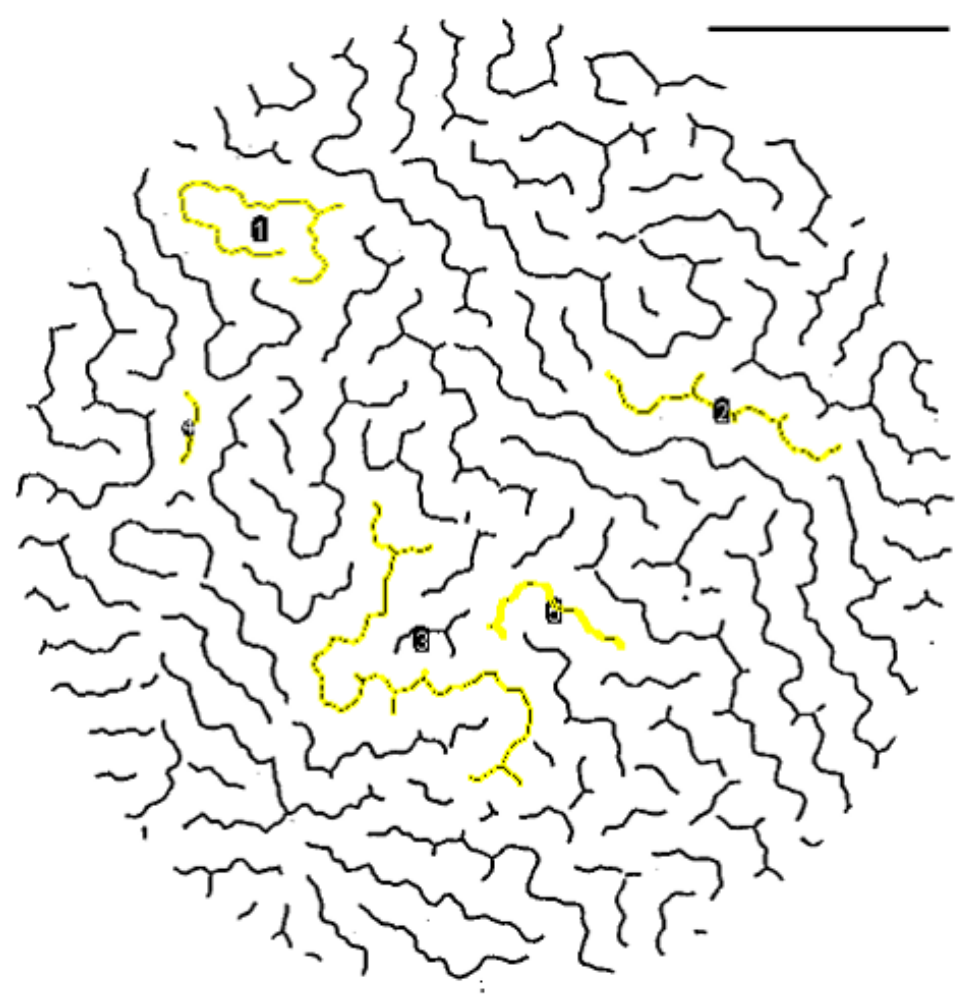

Figure 5.7: Five pillars from core_4c - chamber 10 - slice 40 used to validate measurements of pillars. Scale bar $=0.5 \mathrm{~mm}$. Results given in Table 5.3.

Table 5.3: Measurements of five pillars from core_4c - chamber 10 - slice 40 at two extreme threshold levels compared to threshold level used for quantitative analysis. Difference of lower and upper threshold measurements to used threshold also given.

\begin{tabular}{l|r|rrr|rr} 
Measurement & Pillar \# & \multicolumn{3}{|c|}{ Threshold } & \multicolumn{2}{c}{ Difference } \\
& & Lower & Used & Upper & Lower & Upper \\
\hline \multirow{3}{*}{ Area $\left(\mathrm{mm}^{2}\right)$} & 1 & 0.0080 & 0.0073 & 0.0066 & $9.69 \%$ & $-8.55 \%$ \\
& 2 & 0.0061 & 0.0055 & 0.0050 & $11.32 \%$ & $-8.36 \%$ \\
& 3 & 0.0137 & 0.0123 & 0.0113 & $11.23 \%$ & $-8.41 \%$ \\
& 4 & 0.0016 & 0.0014 & 0.0013 & $12.30 \%$ & $-6.80 \%$ \\
& 5 & 0.0037 & 0.0034 & 0.0032 & $8.60 \%$ & $-8.06 \%$ \\
\hline \multirow{3}{*}{ Perimeter $(\mathrm{mm})$} & 1 & 1.9343 & 1.9249 & 1.9292 & $0.49 \%$ & $0.22 \%$ \\
& 2 & 1.4867 & 1.4824 & 1.4727 & $0.29 \%$ & $-0.65 \%$ \\
& 3 & 3.3184 & 3.3118 & 3.3032 & $0.20 \%$ & $-0.26 \%$ \\
& 4 & 0.3708 & 0.3659 & 0.3599 & $1.32 \%$ & $-1.66 \%$ \\
Feret length (mm) & 5 & 0.9109 & 0.9101 & 0.9094 & $0.08 \%$ & $-0.08 \%$ \\
\hline & 1 & 0.3524 & 0.3524 & 0.3513 & $0.00 \%$ & $-0.30 \%$ \\
& 2 & 0.5189 & 0.5189 & 0.5183 & $0.00 \%$ & $-0.12 \%$ \\
& 3 & 0.6743 & 0.6733 & 0.6733 & $0.15 \%$ & $0.00 \%$ \\
& 4 & 0.1532 & 0.1513 & 0.1494 & $1.28 \%$ & $-1.26 \%$ \\
& 5 & 0.2903 & 0.2899 & 0.2899 & $0.12 \%$ & $0.00 \%$ \\
\hline
\end{tabular}




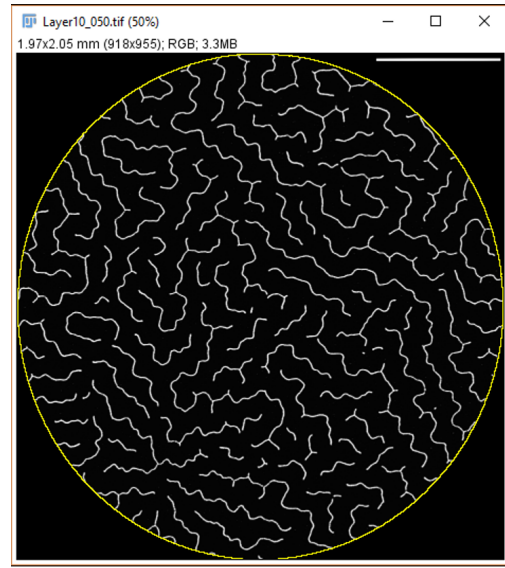

(a)

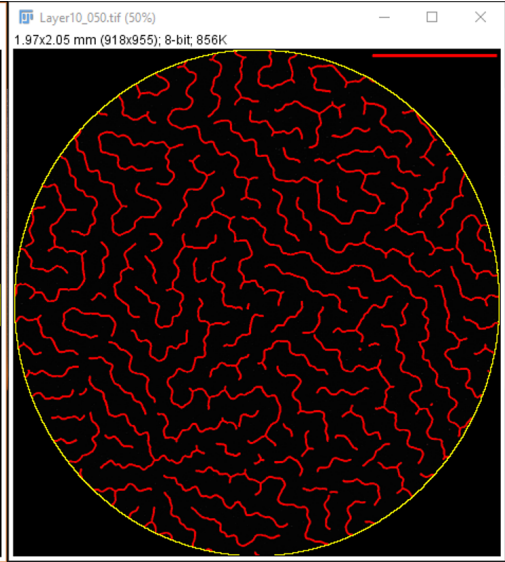

(b)

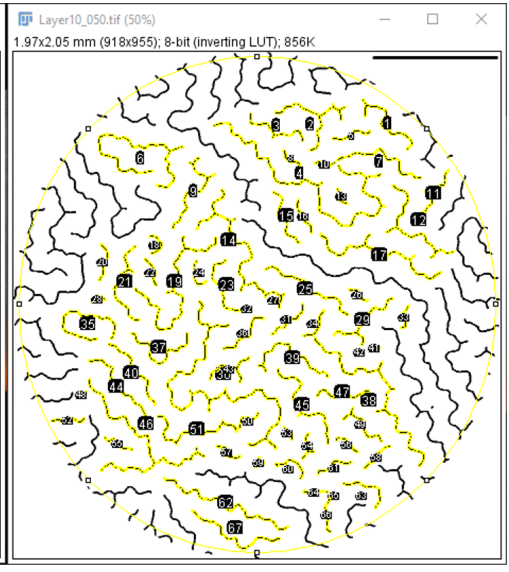

(c)

Figure 5.8: The major steps for preparing slices for analysis in ImageJ. (a) Import raw slice into ImageJ. At this point, a scale is also added, the image is cropped and converted to 8-bit. (b) Threshold applied to pillars to create a binary image. (c) Pillars not in contact with the edge of the sampling area are numbered and considered for analysis.

Figure 5.9 shows how the pillar measurements are calculated. The simplest method to estimate tortuosity is the arc-chord ratio:

$$
\tau=\frac{L}{C}, \quad 1 \leq \tau \leq \infty
$$

where $L$ is length of the curve and $C$ is the distance between the ends of the curve. Therefore $\tau=1$ represents a straight line and $\tau \rightarrow \infty$ represents a loop. From the measurements obtained, the tortuosity of the pillars as a ratio of the skeletonised length of the pillar (assumed as $0.5 \times$ perimeter) and the Feret length can be estimated.

A small proportion of slices were removed from analysis due to a number of reasons:

- Slices taken in proximity to the dorsal shield often contained pillars spanning the length of the sample area. Their true length and other properties were therefore unknown and hence they were removed as they would not give a true representation of their characteristics. Slice (a) in Figure 5.10 shows pillars with low tortuosity at the interface with the dorsal shield. All the pillars are either in contact with the dorsal shield or the edge of the sampling area, and so can't be considered for quantitative analysis. These pillars are instead considered qualitatively later in the study.

- The curved shape of the cuttlebone meant that, although as much care was taken as possible to acquire slices parallel to the septa, some slices still had to be removed from analysis because the slice contained parts of the septa and occasionally adjacent chambers, reducing the sampling area significantly. This was particularly evident in Core_2a since it was taken laterally from the medial axis. The septum can be seen in 


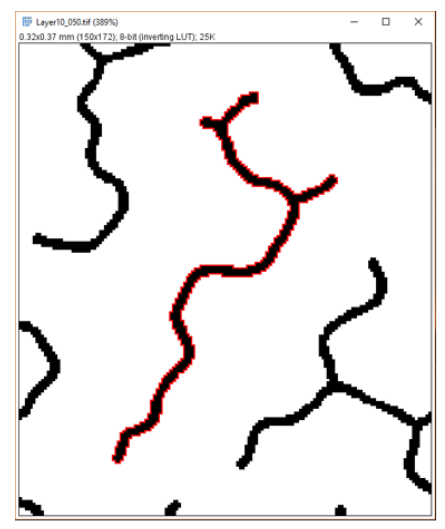

(a)

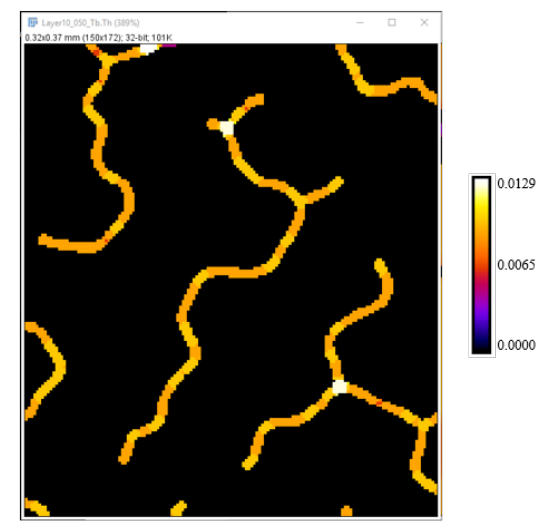

(d)

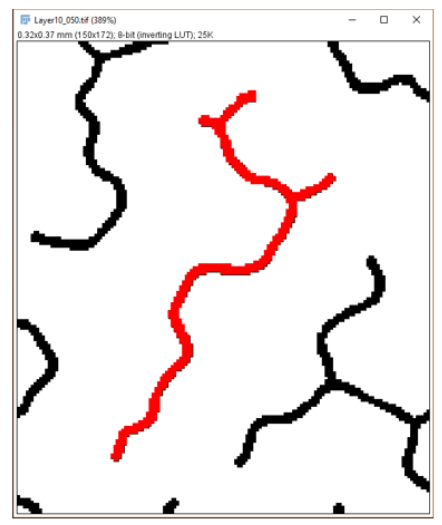

(b)

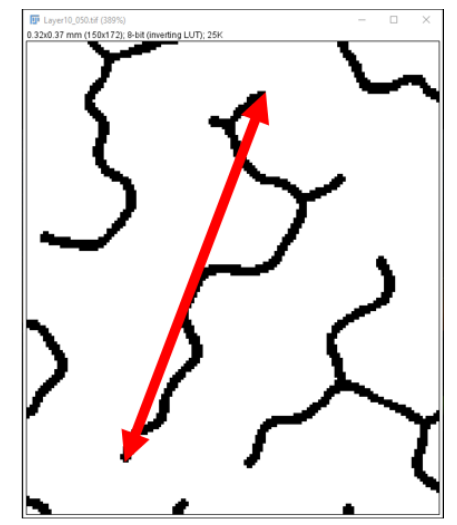

(c)

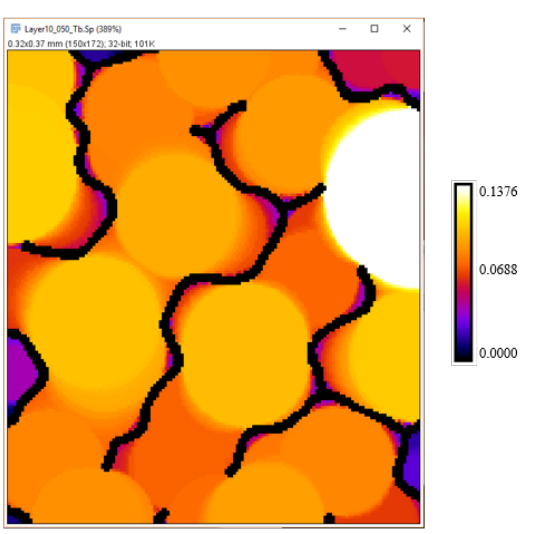

(e)

Figure 5.9: Pillar 9 from core_4c - chamber 10, displaying measurements acquired using ImageJ: (a) perimeter highlighted in red; (b) area, highlighted in red; (c) Feret length, red arrow between two extreme points; (d) pillar thickness, calibration bar in mm; (e) spacing between pillars, calibration bar in $\mathrm{mm}$. 
the white areas of Slice (b) in Figure 5.10. Also, the lower left hand corner of the slice shows the top of chamber 13 .

- Parts of the sampling area of slices, found in chambers towards the ventral edge (typically the posterior, siphonal region), may not contain any data as the chamber ends. Therefore results such as pillar counts would be skewed. In this case, the slices would be removed. Slice (c) in Figure 5.10 shows the most ventral chamber in core_5c. The chamber covers less than half of the sampling area since the chamber ends.

$\mathrm{X}$-ray $\mu \mathrm{CT}$ allows for both quantitative and qualitative analysis of natural specimens. Quantitative analysis provides a good foundation of a comprehensive description of the cuttlebone, however qualitative analysis is effective in providing information which can be difficult to quantify. For example, Figure 5.8(c) shows that a significant proportion of pillars in the particular sample are not considered for quantitative analysis since they are in contact with the edge of the sampling area. Qualitative analysis allows for the nature of these dropped pillars to be considered, and is included in this study.

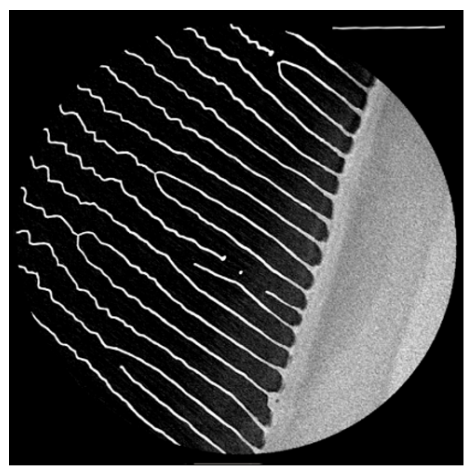

(a)

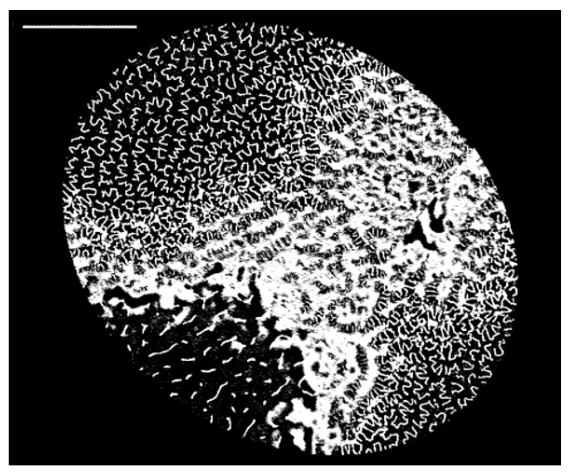

(b)

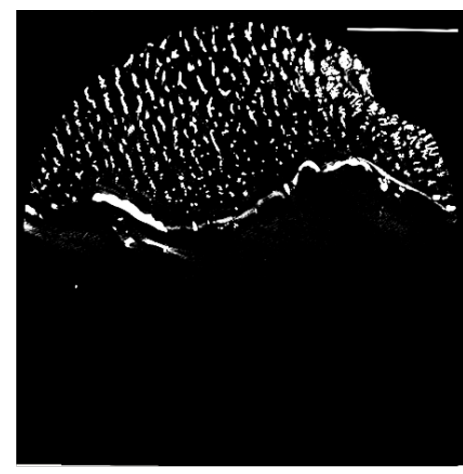

(c)

Figure 5.10: Examples of slices removed from analysis: (a) core_2b - chamber 2 - slice 60; (b) core_2a - chamber 12 - slice 49; (c) core_5c - chamber 24 - slice 00. Scale bar $=0.5 \mathrm{~mm}$.

\subsubsection{Scanning Electron Microscopy}

Specimens of cuttlebone were imaged using a range of scanning electron microscopes (SEMs) described in Table 5.4. Samples were fractured to reveal areas of interest and mounted either directly onto an aluminium stub using carbon adhesive and silver paint, or in a clamp. Samples mounted in resin and those not imaged in environmental (or variable pressure) and gentle-beam modes, required a conductive coating of carbon prior to image acquisition.

A range of accelerating voltages were used for acquisition of images. For conventional secondary electron (SE) and backscattered electron (BSE) imaging, $15-20 \mathrm{kV}$ was applied, whilst in gentle-beam and low $\mathrm{kV}$ modes, $0.8-1.5 \mathrm{kV}$ was used. 
Table 5.4: Imaging modes for all scanning electron microscopes used. * Where not stated, all systems are fitted with standard secondary and backscattered electron detectors. VPSE variable pressure secondary electron, $E B S D$ - electron backscatter diffraction, $E D S$ - electron dispersive spectroscopy, $L E D$ - lower electron detector.

\begin{tabular}{llll} 
Machine & Imaging mode & FIB & Detectors used \\
\hline Zeiss Evo LS & environmental & - & VPSE \\
Zeiss Cross Beam 540 & conventional & Ga+ ion beam & EBSD \\
JEOL FEG 7800F & gentle-beam & - & LED, EBSD, EDS \\
FEI Quanta FEG & environmental / low kV & - & $*$ \\
FEI/Philips XL30 FEG & environmental & - & $*$ \\
\hline
\end{tabular}

\subsubsection{Electron Backscatter Diffraction}

Electron backscatter diffraction (EBSD) maps were acquired with the help of Dr. Mark Coleman at Swansea University. EBSD analysis in this study was conducted using a JEOL 7800F FEG SEM equipped with a Nordlys Nano EBSD detector and a Zeiss Cross Beam 540 SEM with a Nordlys 2 EBSD detector. EBSD data was collected using Oxford Instruments AZtec software. Data was then exported to HKL Channel 5 software with maps and pole figures created in Tango and Mambo respectively.

The iterative process required for the preparation of cuttlebone for EBSD analysis is illustrated as a workflow in Figure 5.11. Samples of cuttlebone were either embedded in epoxy resin or left unmounted. Samples were ground and polished (as per the process described in section 3.4.2 and in [222]) to a $0.03 \mu \mathrm{m}$ finish, with colloidal silica and then cleaned. Observational checks were made to see if the sample surface was free of topography. If the sample appeared acceptable for EBSD analysis, a carbon layer was applied and a map was attempted. For all other samples, both focussed ion beam (FIB) and broad ion beam (BIB) milling techniques were applied to remove any remaining topography and surface damage from manual polishing, before carbon coating. The recommended coating thickness of $2.5 \mathrm{~nm}[140,222]$ was not reducing charging effects, therefore various coating thicknesses were tested.

The final implemented sample preparation process for the acquisition of EBSD maps is summarised as;

- embed sample in epoxy resin,

- manual polishing to $0.03 \mu \mathrm{m}$,

- broad beam milling at $2 \mathrm{kV}$ and $80 \mu \mathrm{A}$ for approximately 10 hours,

- carbon coat with thickness of 6-7 $\mathrm{nm}$.

A range of acquisition parameters were tested for optimal Kikuchi patterns; accelerating voltage, probe current, working distance, beam aperture, camera binning, exposure, frame 


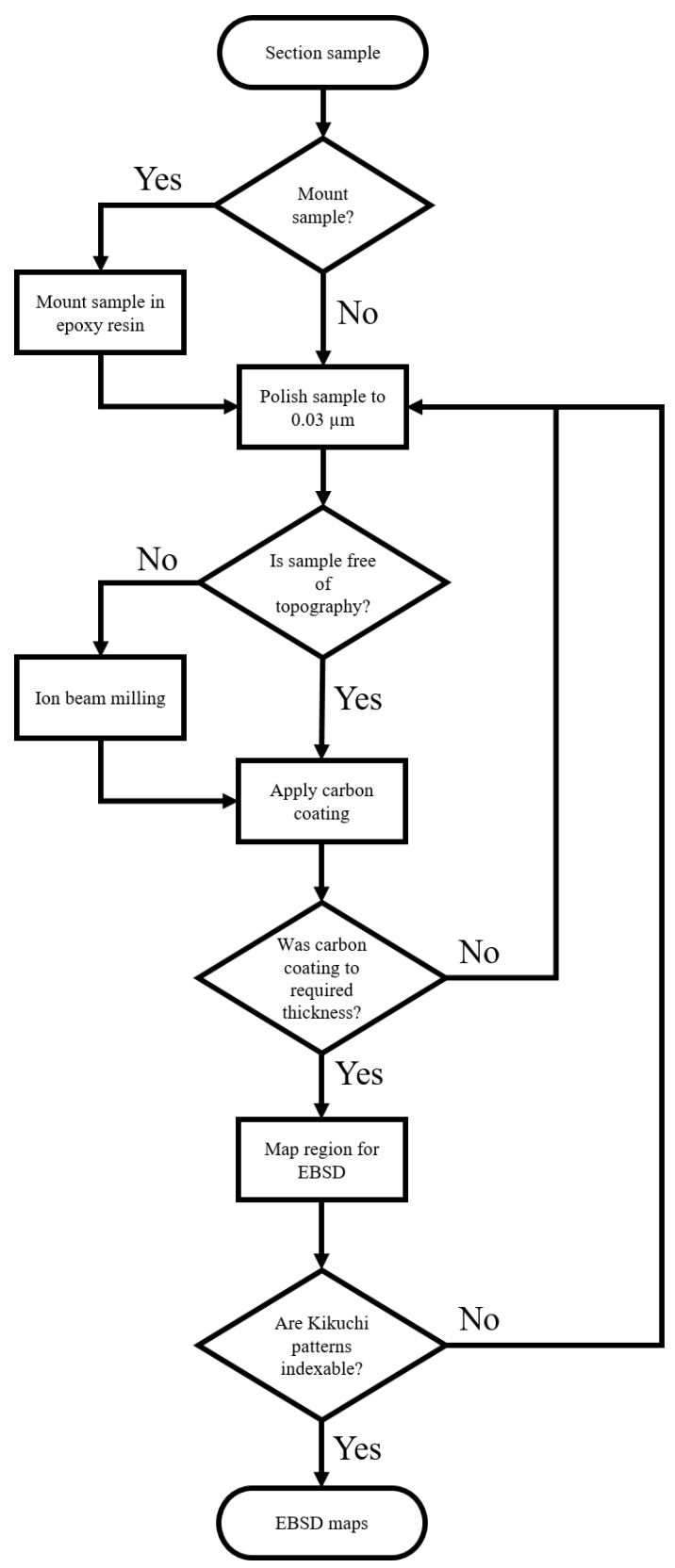

Figure 5.11: Flow chart of iterative process used to produce EBSD maps of cuttlebone. 
average and Hough parameters. Kikuchi patterns produced were indexed using the AZtecHKL database which contains the structure file for aragonite. EBSD maps were aligned according to the septum being parallel to the $\mathrm{x}$-direction. Final map acquisition settings are given in Table 5.5.

Table 5.5: EBSD map acquisition settings. Acc. V - accelerating voltage, WD - working distance, Exp. - exposure, Frame Av. - frame averaging.

\begin{tabular}{llrrrrrrr} 
Region & System & $\begin{array}{r}\text { Acc. V } \\
(\mathrm{kV})\end{array}$ & $\begin{array}{r}\text { WD } \\
(\mathrm{mm})\end{array}$ & $\begin{array}{r}\text { Step size } \\
(\mathrm{nm})\end{array}$ & $\begin{array}{r}\text { Rate } \\
(\mathrm{pps})\end{array}$ & $\begin{array}{r}\text { Binning } \\
\text { Exp. } \\
\text { ms }\end{array}$ & Frame Av. \\
\hline Septum & JEOL 7800F & 14.5 & 17 & 100 & 2.254 & $2 \times 2$ & 220 & 2 \\
Shield & Zeiss C-B 540 & 12 & 11.6 & 500 & 0.849 & $2 \times 2$ & 270 & 2 \\
\hline
\end{tabular}

\subsubsection{Chemical analysis}

This section describes the methods used to analyse the chemistry of the cuttlebone: energy dispersive X-ray spectroscopy and X-ray photoelectron spectroscopy.

\subsubsection{Energy Dispersive X-ray Spectroscopy}

EDS analysis was carried out on regions of cuttlebone sectioned in the transverse plane (see Figure 5.3), to provide chemical analysis on both the dorsal shield and lamellar matrix. Sections were embedded in epoxy resin using vacuum impregnation, in order to infiltrate the complex porosity network, and polished to $0.3 \mu \mathrm{m}$. The samples were then carbon coated to ensure an optimum number of X-ray counts were produced.

Sites of interest were identified and elemental mapping was conducted as well as line scans to monitor any changes in chemistry across regions. EDS analysis was performed at $20 \mathrm{kV}$ using a JEOL 7800F FEG SEM with Aztec software (Oxford Instruments), equipped with a SMax50 EDS detector.

\subsubsection{X-ray Photoelectron Spectroscopy}

To examine the stoichiometry of the cuttlebone, X-ray Photoelectron Spectroscopy (XPS) spectra and maps were obtained using a Kratos Axis Supra system utilising a 15 kV 15 mA monochromated $\mathrm{Al} \mathrm{K}_{\alpha} \mathrm{X}$-ray source.

Cuttlebone samples were cut along the transverse axis and polished to minimise topography in order to achieve a high photoelectron count.

To understand the elemental distribution, XPS images were acquired using an achromatic $\mathrm{Al} \mathrm{K}_{\alpha}$ flood source with equivalent power. Wide low resolution spectra were first recorded up to a binding energy of $1200 \mathrm{eV}$ using a magnetic-electrostatic hybrid lensing system and a $700 \times 300 \mu \mathrm{m}$ slot collimator, with pass energy of $160 \mathrm{eV}$, dwell time of 100 
ms and a step size of $1 \mathrm{eV}$. High resolution core levels were measured using the same lens and collimator as low resolution spectra, with a pass energy of $20 \mathrm{eV}$, a dwell time of $250 \mathrm{~ms}$ and a varying number of averaged scans depending on the signal-to-noise ratio of individual peaks. A filament and magnetic confinement-based charge neutraliser was used to account for charging effects. The measured binding energies were calibrated using the adventitious C1s peak with a binding energy value of $284.8 \mathrm{eV}$. High resolution XPS images were recorded in $400 \times 400 \mu \mathrm{m}$ and $200 \times 200 \mu \mathrm{m}$ fields of view, with a pass energy of $160 \mathrm{eV}$ and a dwell time of $600 \mathrm{~s}$. To account for background signal, images were first recorded at the point of greatest peak intensity. Additional scans then captured the background signal pre and post peak, which were subtracted from the peak images using a linear fit.

XPS analysis was carried out by Dr. James McGettrick at Swansea University.

XPS theory XPS is a spectroscopic method that uses X-ray radiation to irradiate a material and records the number and energy of electrons released from the sample surface. An X-ray photoelectron spectrometer consists of an X-ray source, an ultra-high vacuum chamber, collection lenses and a hemispherical electron analyser, shown in Figure 5.12.

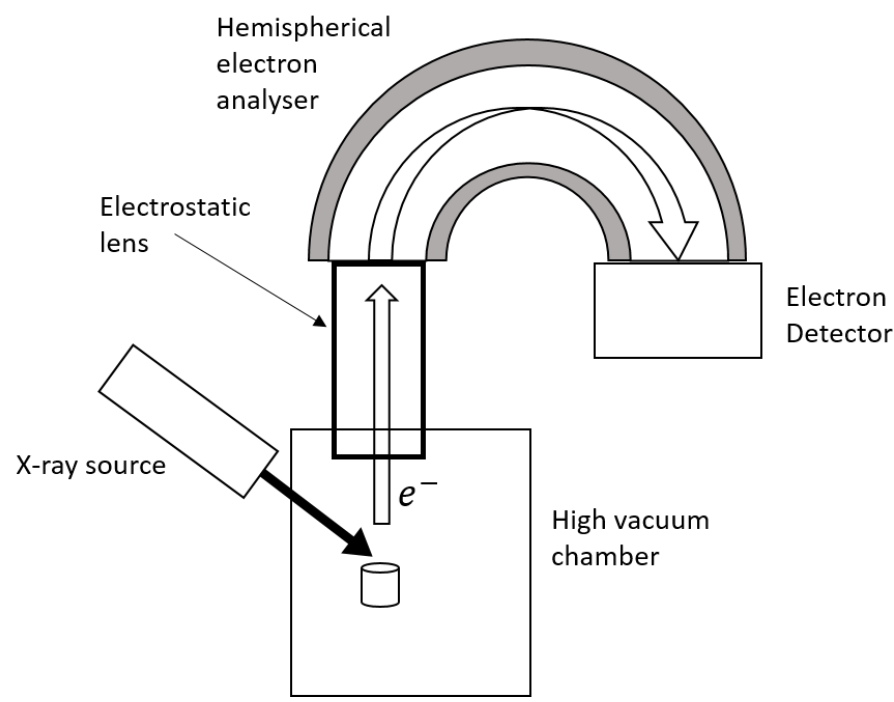

Figure 5.12: XPS schematic.

A monochromatic beam is required in order to obtain accurate readings. The beam is focused on the surface of the sample where it encourages the emission of electrons by exciting atoms with X-ray bombardment. The kinetic energy of an electron is dependent on the photon energy $(h v)$ and the binding energy of the electron. This technique is used to measure the type and quantity of surface elements, as well as the chemical states and binding energies of surface atoms. Collected electron binding energies form a spectrum, as shown in Figure 5.13, where binding energy is plotted against the intensity of the energy.

In addition to the acquisition of spectra, XPS can be used to image the elemental 


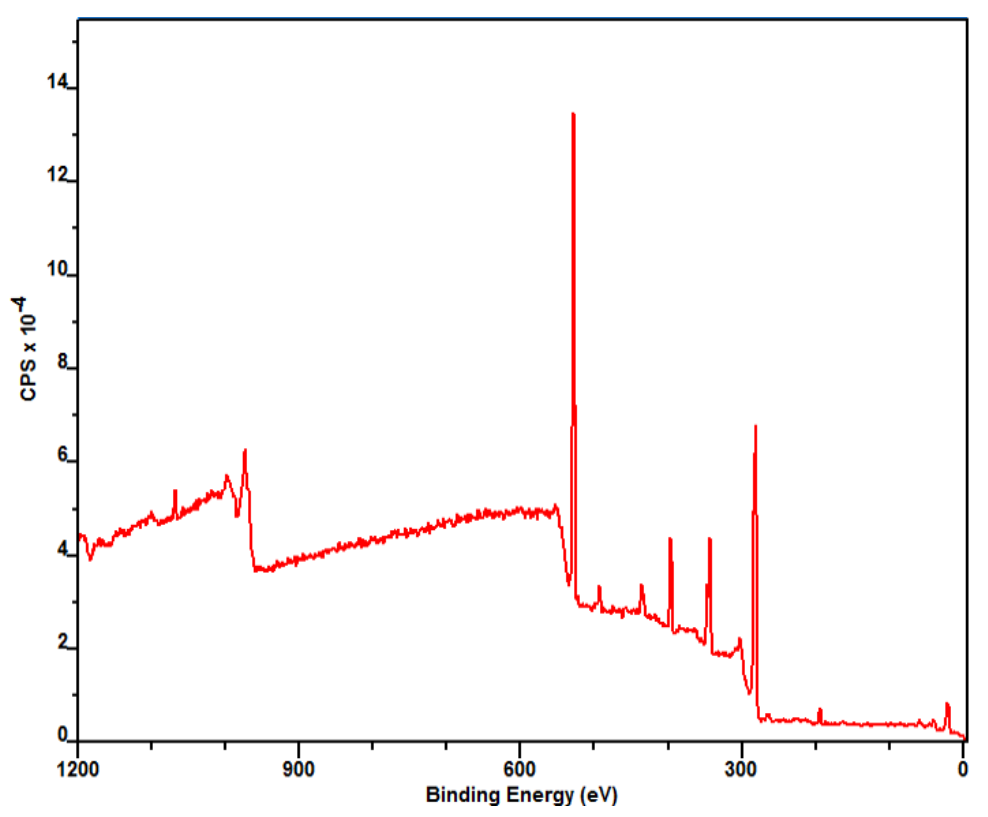

Figure 5.13: XPS wide spectrum of cuttlebone.

distribution across a two-dimensional area.

\subsubsection{Mechanical testing}

This section describes the methods used to test the mechanical properties of the cuttlebone: compression testing and nanoindentation.

\subsubsection{Compression testing}

$\mathrm{X}$-ray $\mu \mathrm{CT}$ is increasingly used for the acquisition of $3 \mathrm{D}$ images as it allows the internal structure of a specimen to be reconstructed non-destructively. This makes it an ideal means of following structural development through time-lapse imaging. X-ray CT with repeated time-lapse therefore allows the user to follow the evolution of a structure under a controlled environment. Studying how the cuttlebone deforms mechanically in three dimensions when subjected to a compressive load can help inform form and function. The aim of this analysis is to visualise the behaviour of the internal structure of cuttlebone under sequential compressive loading. Sections of cuttlebone are loaded progressively and a series of 3D images are obtained using X-ray microtomography. This produces a time-lapse series allowing structural deformation to be visualised.

Samples of cuttlebone were obtained from dried samples of Sepia officinalis. The samples were used to investigate bulk deformation. Carbon fibre tubes of $5 \mathrm{~mm}$ diameter were used to produce cylindrical cores. Carbon fibre was used as it has a low X-ray attenuation coefficient, allowing X-rays to pass through easily with minimal impact on the resulting $\mathrm{X}$-ray CT data. This also allowed the cored samples of cuttlebone to fit directly onto the 
specifically designed novel rig $^{1}$ [223] shown in Figure 5.14, as well as giving an isotropic voxel size of $6 \mu \mathrm{m}$.

The novel rig was manufactured using PLA and 3D printing technology. This allowed the specimens to be transferred between the compression testing machine and X-ray CT scanner with minimal disruption to the samples. It was designed so that the specimens would maintain the same position throughout, ensuring alignment between scans.

Loading was applied perpendicular to the septa and performed on a uniaxial compression testing machine in accordance with EN ISO 7500-1 Class 2.5, with a load cell of $25 \mathrm{kN}$. A stainless steel compression platen was used with a displacement rate of $0.25 \mathrm{~mm} / \mathrm{min}$.

A reference scan was first taken of the samples. When local peak loads were achieved, loading was removed and the samples were scanned. The process was repeated until enough data was collected and a pattern of the structural deformation was evident.

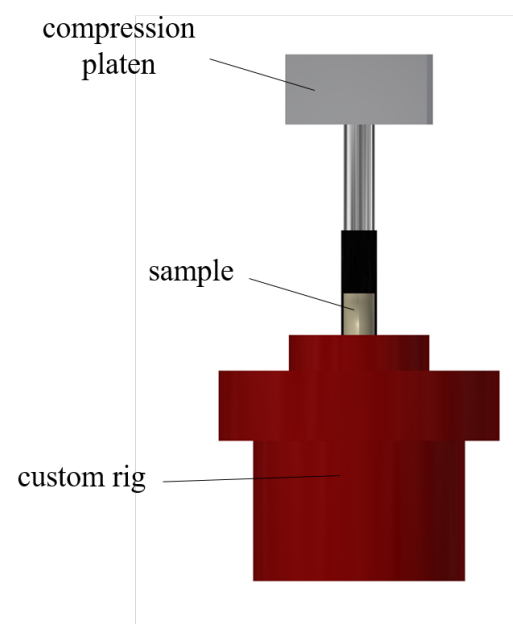

Figure 5.14: Schematic of compression testing.

\subsubsection{Nanoindentation}

Nanoindentation of embedded cuttlebone samples was carried out by Dr. David Labonte in Dr. Michelle Oyen's Laboratory at the Department of Engineering, Cambridge University. Samples of freshly dissected cuttlebone were sectioned along the transverse and dorsoventral planes (see Figure 5.3). These were mounted in epoxy resin using vacuum impregnation, left to cure for 24 hours and polished to $0.3 \mu \mathrm{m}$. Indentation was carried out on areas of the dorsal shield, septa and individual pillars as shown in Figure 5.15.

Hardness and elastic modulus were acquired by nanoindentation (UBI-1, Hysitron) with a Berkovich tip. Indentation was carried out with a trapezoidal loading profile consisting of a 5 second loading to a peak load of $6 \mathrm{mN}$, a 10 second hold phase at the peak load of $6 \mathrm{mN}$, and an unloading time of 5 seconds. The tests were performed at least 10 times on

\footnotetext{
${ }^{1}$ This file has been made publicly available via figshare.com and interfaces with a Nikon XT H 225.
} 


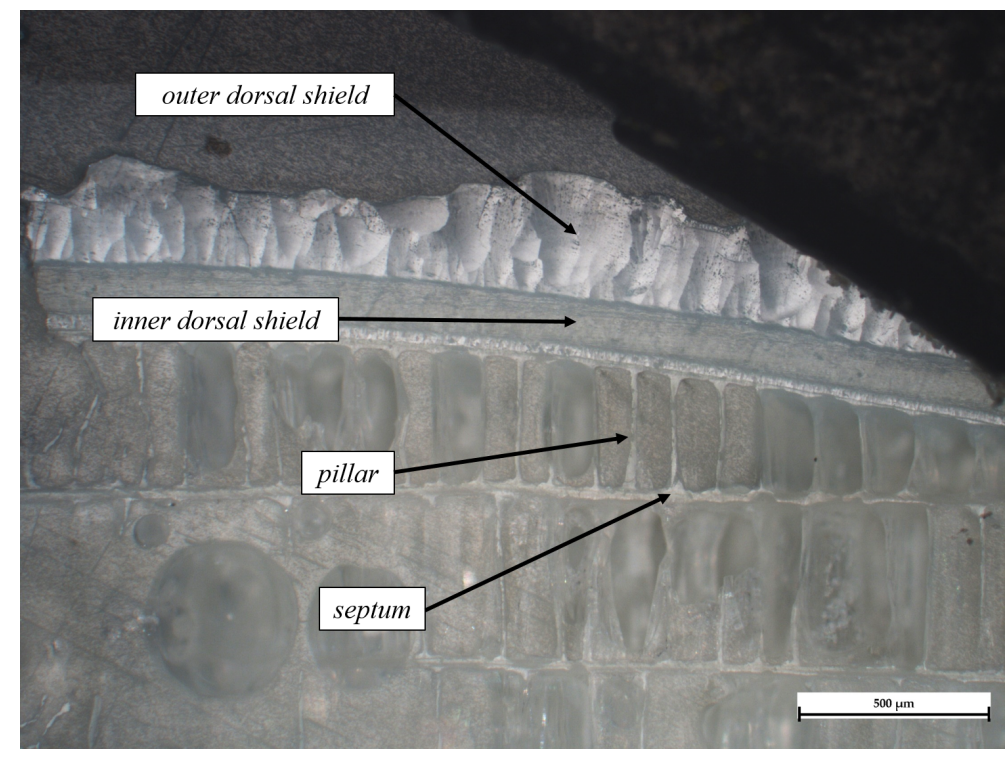

Figure 5.15: Example of area for nanoindentation.

each region and the average value and standard deviation were calculated. The material properties were determined with the Oliver-Pharr method [154] (see section 3.5.1).

\subsubsection{3D printing and compression testing}

To gain insight into the mechanical properties of different regions and the failure mechanism of cuttlebone, prototype geometries of chamber sections were created using additive layer manufacturing. Regions of differing pillar morphology were identified from structural characterisation carried out during this work. Three areas of focus were chosen as initial investigations using a simplistic approach to attempt to link form and function;

- the effect of root-like structures at chamber floor and ceiling,

- the impact of pillar morphology within a chamber on compressive strength, and

- comparing the progressive failure mechanism of cuttlebone versus a honeycomb cellular structure.

The three-layer honeycomb structure and filleted and non-filleted pillar structures were created using SolidWorks (see Appendix A for sketches). A fillet radius was applied to mimic the root-like structures found at the chamber extremes. All regions taken directly from cuttlebone were extracted from $\mu \mathrm{CT}$ data. For the pillar morphology comparisons, regions of interest of $1 \mathrm{~mm}^{2}$ that captured full chamber height (including septa) were used. For the progressive failure sample, three full chambers were extracted with a region of interest of $1 \mathrm{~mm}^{2}$. Region of interests of $1 \mathrm{~mm}^{2}$ were used to optimise the trade-off between number of pillars captured and print limitations (i.e. pillar thickness, layer height and sample size). 
Cross-sections of STL files are given in Figure 5.16 and their reference positions for the regions are given in Table 5.6. These sections were chosen because they display high variations in pillar morphology. The regions were meshed, cut to give flat parallel edges enabling good contact with the compression platens, scaled (x50), and 3D printed. The meshes were cleaned in Geomagic, any holes were filled, and the mesh was smoothed and decimated to reduce printing time without impacting the pillar morphology. They were printed with PLA on the Ultimaker ${ }^{2}$. Sample dimensions and print settings are given in Table 5.7. To minimise variations in the additively manufactured structures, infill was set to $100 \%$ and print settings were consistent across comparable samples.

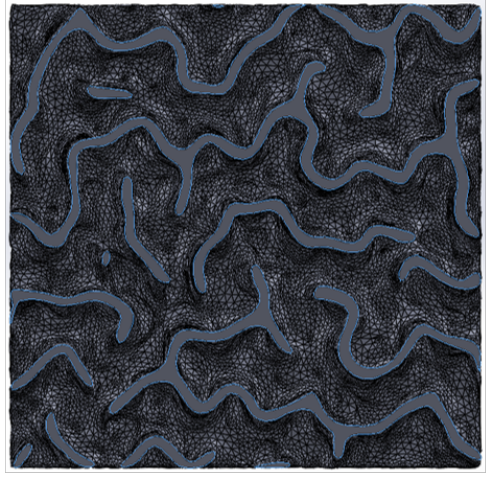

cham 1

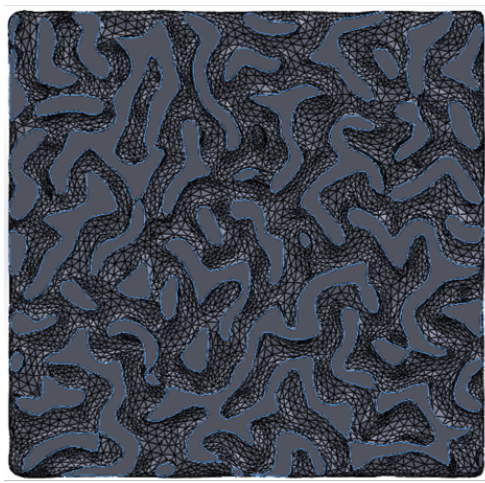

cham 3

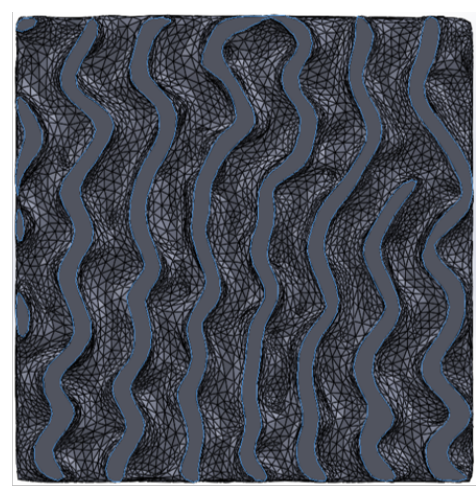

cham2

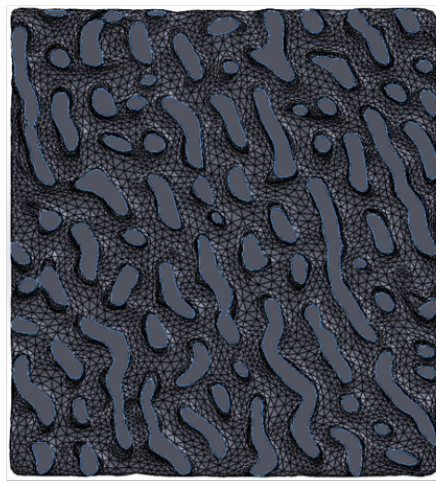

cham4

Figure 5.16: Cross-section at mid-point of single chamber STL files used in compression analysis.

Compression testing was performed on a uniaxial compression testing machine in accordance with EN ISO 7500-1 Class 2.5, with load cells of $5 \mathrm{kN}$ and $50 \mathrm{kN}$. Samples were loaded perpendicular to the septa and settings for each sample are given in Table 5.7.

\subsection{Results and discussion}

The following section is split into three main areas of results; structural, chemical and mechanical. Analysis and discussion of the results is provided in each section. 
Table 5.6: Reference positions of samples taken directly from CT data with respect to longitudinal, transverse and dorsoventral axes. MtAP $=$ Material-to-air percentage.

\begin{tabular}{ccccl} 
Sample & $\begin{array}{c}\text { Longitudinal Axis } \\
\text { \% total length }\end{array}$ & $\begin{array}{c}\text { Transverse Axis } \\
\text { \% total height }\end{array}$ & $\begin{array}{c}\text { Dorsoventral Axis } \\
\text { \% total depth }\end{array}$ & Description \\
\hline cham1 & 10 & 50 & 50 & $\begin{array}{l}\text { Avg. pillar tortuosity, } \\
\text { low MtAP }\end{array}$ \\
cham2 & 25 & 50 & 20 & $\begin{array}{l}\text { Low pillar tortuosity } \\
\text { cham3 }\end{array}$ \\
High pillar tortuosity, \\
cham4
\end{tabular}

\subsubsection{Structural analysis}

Structural analysis of cuttlebone considers morphology using 3D data, microstructure, and crystallography. The results of each of these analyses are reviewed in this section.

\subsubsection{Morphological features}

Quantitative analysis was carried out on the morphological features of the cuttlebone. The aim being to characterise the morphology across the cuttlebone in 3D as this has not been done previously. Other reports of cuttlebone morphology have not taken into account any changes that occur across the cuttlebone in pillar structure. Pillars are usually documented as having long, sinusoidal shapes that run anteroposteriorly [184, 192, 213], with observations being based on visible surfaces, even when cut or fractured.

3D data and images of cores from the cuttlebone (see Figure 5.4) were obtained using Xray $\mu \mathrm{CT}$. Individual pillar level data was created, giving the ability to group and summarise results at a pillar, slice, chamber or core level. This is the first comprehensive characterisation of the morphology variation across the internal cuttlebone of Sepia officinalis in three dimensions.

A brief overview of all the cores is given before focusing in detail on three interesting aspects of the dataset; pillar counts, material-to-air percentage and pillar tortuosity.

The charts in Figure 5.17 represent a slice count for each chamber of each core analysed. The bars are coloured based on the number of slices included and disregarded from analysis. There are a number of features to note in this summary:

- The number of chambers per core differs depending on the position of the core in the cuttlebone. Core_4c, for example, has the highest number of chambers with 33, as it was taken from the thickest part of the structure (in the dorsoventral direction), whilst 


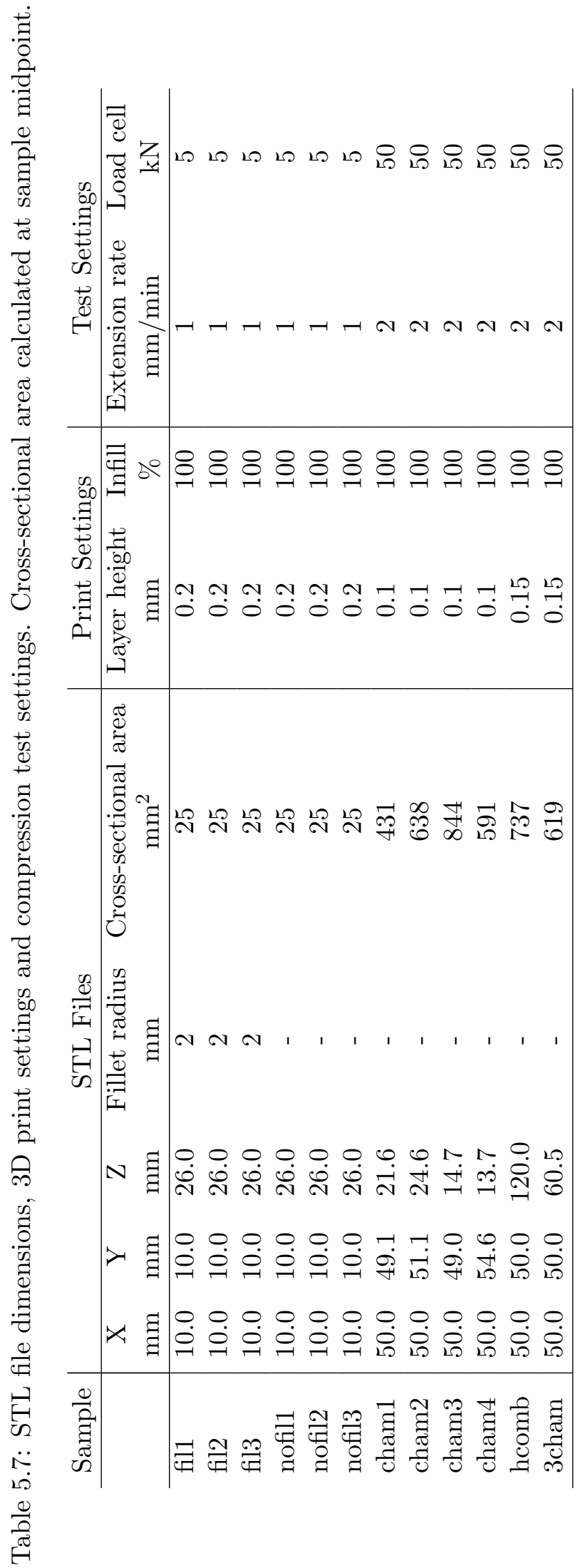


Table 5.8: A summary of the number of chambers, and the minimum and maximum complete chambers for each core.

\begin{tabular}{lrrr} 
Core & Total Chambers & Min Chamber & Max Chamber \\
\hline core_1b & 9 & 2 & 9 \\
core_2a & 14 & 4 & 14 \\
core_2b & 18 & 3 & 18 \\
core_3c & 24 & 2 & 24 \\
core_4c & 33 & 2 & 32 \\
core_5c & 24 & 3 & 22 \\
core_6b & 15 & 3 & 12 \\
\hline
\end{tabular}

core_1b only has 9 chambers because it was taken from the most anterior region. By multiplying the total number of slices by the slice interval of $0.004 \mathrm{~mm}$, the chamber heights can be estimated. The chamber height reduces towards the ventral edge of the cuttlebone.

- The distributions of slice count across chambers are generally smooth and consistent across cores. All cores, particularly the anterior cores 1b, 2a, 2b, 3c show an increase in slice count in the most ventral chamber, compared to those chambers immediately preceding. This feature of the cuttlebone can be seen in the schematic in Figure 5.4. The most ventral chamber of these cores is the last to be mineralised, which could be a factor in this feature.

The process for excluding slices is not systematic but is consistent based on the three criteria described in section 5.2.2.2 and illustrated in Figure 5.10. The prevalence of each type of slice rejection criteria is different in each core, therefore the proportion of disregarded slices is not consistent across cores. For example, the dorsal shield intersects more chambers in core_2a because of its position relative to the medial axis (compared to the other cores) and the curvature of the septa being more pronounced at the lateral edges. The impact of disregarding slices is that a limited number of regions are not fully represented in the results, however these regions only make up a small proportion of the whole cuttlebone. Due to the curved shape of the cuttlebone, the sampling area of some chambers in proximity to the dorsal shield, and the most ventral chambers, doesn't always solely contain pillars. For example, the 3D cores in Figure 5.4 show that the dorsal shield often intersects the first few chambers, and chambers in proximity to the ventral surface in cores $4 \mathrm{c}, 5 \mathrm{c}$ and $6 \mathrm{~b}$ only partially span the width of the sampling area. Table 5.8 contains a summary of the number of complete chambers in each core.

Three types of pillar measurement from the data are now analysed in detail across the cores. These measurements are then used to make some general suggestions about morphological features of the pillars across the entire cuttlebone.

Pillar counts for each slice and chamber are plotted as a series of lines, with a separate 


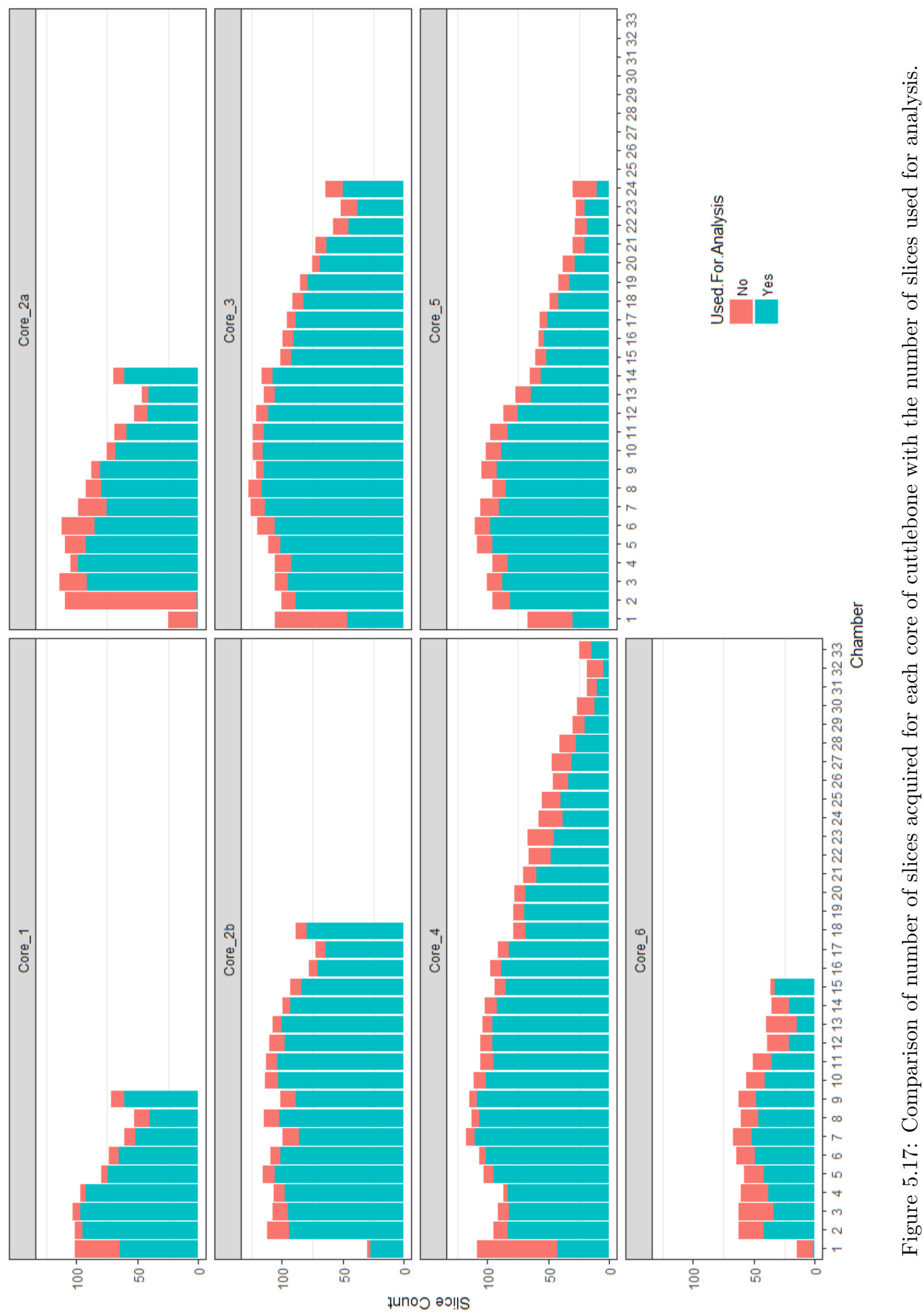


figure for each core. From initial observations of the 3D data, it is clear that the number of pillars varies greatly, both within a core, and across sampling positions.

The material-to-air percentage is calculated by specifying a region of interest in each slice, and then measuring material at a given threshold. The area, thickness and spacing of the pillars all combine to influence this measurement. These results are presented in the same way as the pillar count results, in a series of line graphs.

The tortuosity of the pillars is calculated using equation (5.2.2.1). Previous studies have identified a change in shape of the pillar at the dorsal and ventral parts of an individual chamber [184], however a measure of pillar tortuosity across the cuttlebone has never been achieved. Again, these results are also given as a series of line graphs.

For a more general view, average values of these measurements for each chamber in each core are given in Figures 5.46 and 5.47.

An example is given to illustrate these measurements for a specific chamber of the cuttlebone specimen, before analysing all chambers of each core.

Example 5.3.1.1. Core_4c - chamber 10 Six evenly spaced slices from core_4c - chamber 10 are selected. The images in Figure 5.18 show original CT data for each slice and summary data is given in Table 5.9. The images show the incredible diversity of form which can be found in just one chamber in one core from roof to floor.

Table 5.9: A summary of pillar counts and average pillar measurements of six slices from chamber 10 of core_4c. Perim. = average perimeter, Feret. = average Feret length, Tort. = average tortuosity, Th. = average thickness, Sp. = average spacing, Mat. to Air $=$ average material-to-air percentage.

\begin{tabular}{lrrrrrrrr} 
Slice & Count & $\begin{array}{r}\text { Area } \\
\left(\mathrm{mm}^{2}\right)\end{array}$ & $\begin{array}{r}\text { Perim. } \\
(\mathrm{mm})\end{array}$ & $\begin{array}{r}\text { Feret. } \\
(\mathrm{mm})\end{array}$ & Tort. & $\begin{array}{r}\text { Th. } \\
(\mathrm{mm})\end{array}$ & $\begin{array}{r}\text { Sp. } \\
(\mathrm{mm})\end{array}$ & $\begin{array}{r}\text { Mat. to Air } \\
\%\end{array}$ \\
\hline 000 & 33 & 0.0038 & 0.9455 & 0.2622 & 1.5471 & 0.0099 & 0.0802 & 10.5238 \\
020 & 44 & 0.0027 & 0.7546 & 0.2351 & 1.4086 & 0.0089 & 0.0869 & 8.4489 \\
040 & 58 & 0.0024 & 0.6623 & 0.2163 & 1.3974 & 0.0090 & 0.0833 & 8.9581 \\
060 & 58 & 0.0026 & 0.7267 & 0.2246 & 1.4768 & 0.0089 & 0.0787 & 9.2484 \\
080 & 62 & 0.0026 & 0.7690 & 0.2177 & 1.6130 & 0.0087 & 0.0708 & 10.0459 \\
100 & 113 & 0.0021 & 0.6931 & 0.1724 & 1.8155 & 0.0082 & 0.0548 & 11.8437 \\
\hline
\end{tabular}

Pillar count Vertical pillars are joined to contiguous septa to form each chamber. It is therefore expected that the pillar count taken across slices in one chamber would remain the same. However, from Table 5.9 in Example 5.3.1.1, it can be seen that the number of pillars recorded in slice_100 is 113 which is more than triple the pillar count in slice_000 (33). Clearly then, in the example at least, pillar count is not consistent throughout an individual chamber.

Figures 5.19-5.25 show pillar counts recorded on each slice for each chamber in each sample core. 

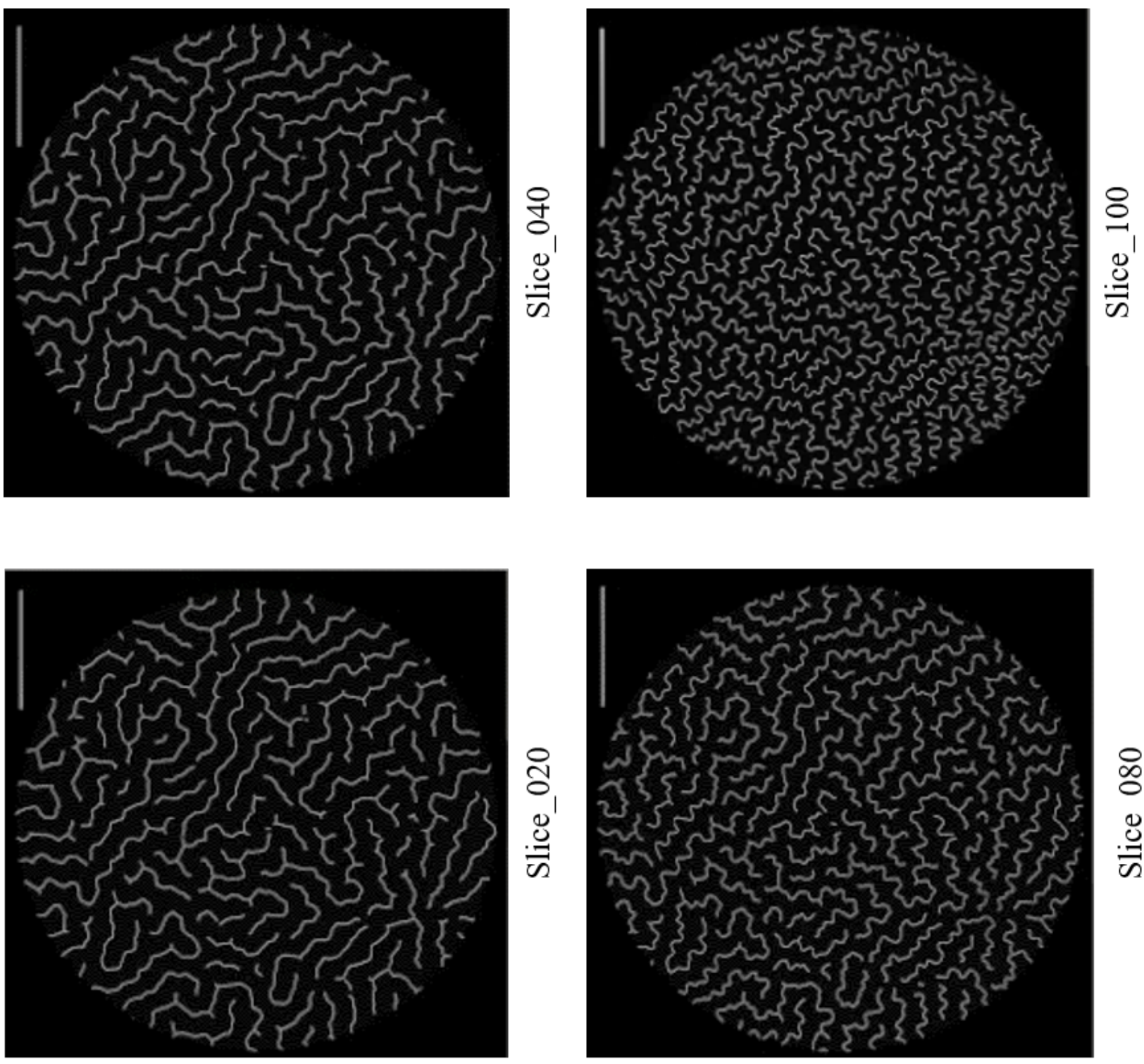

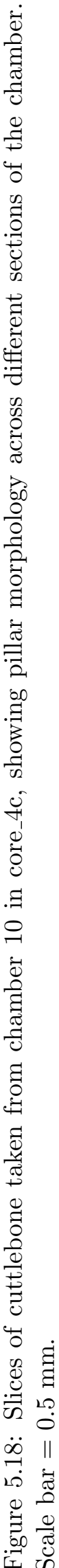
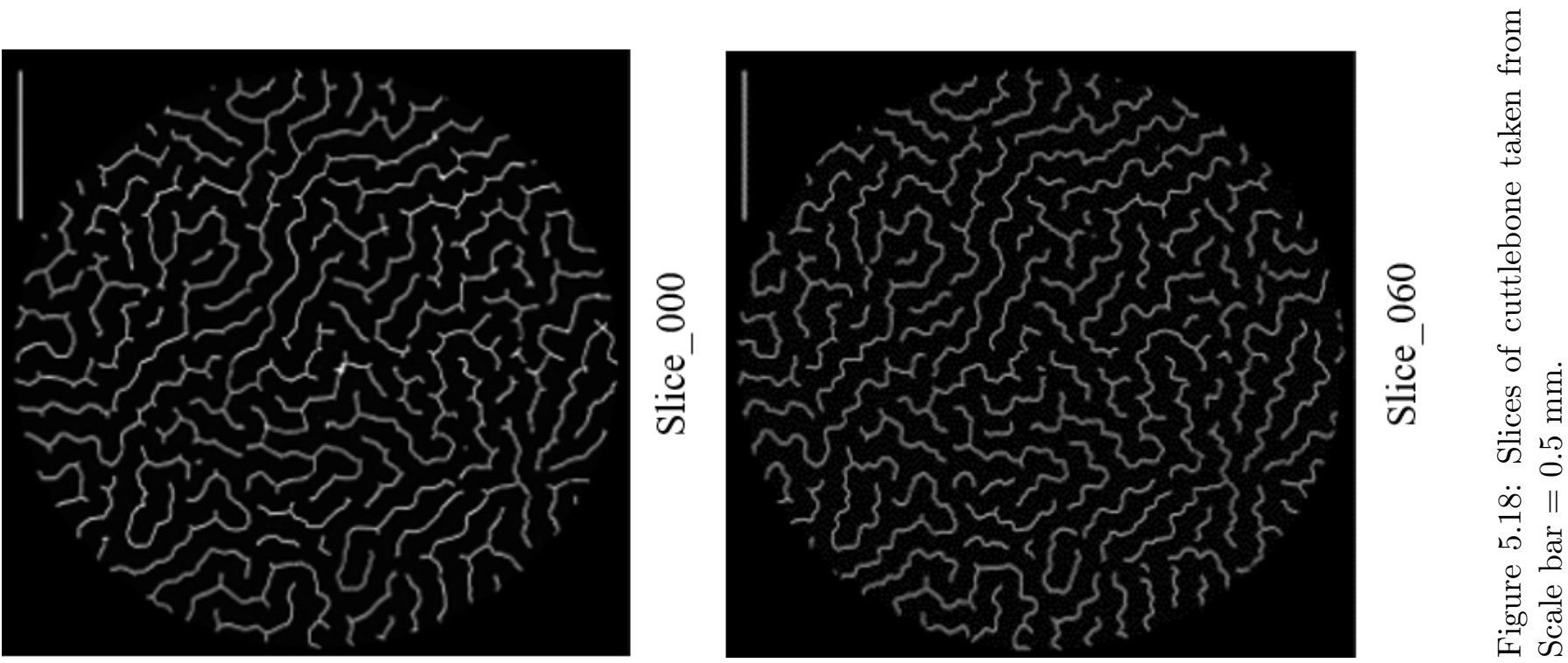


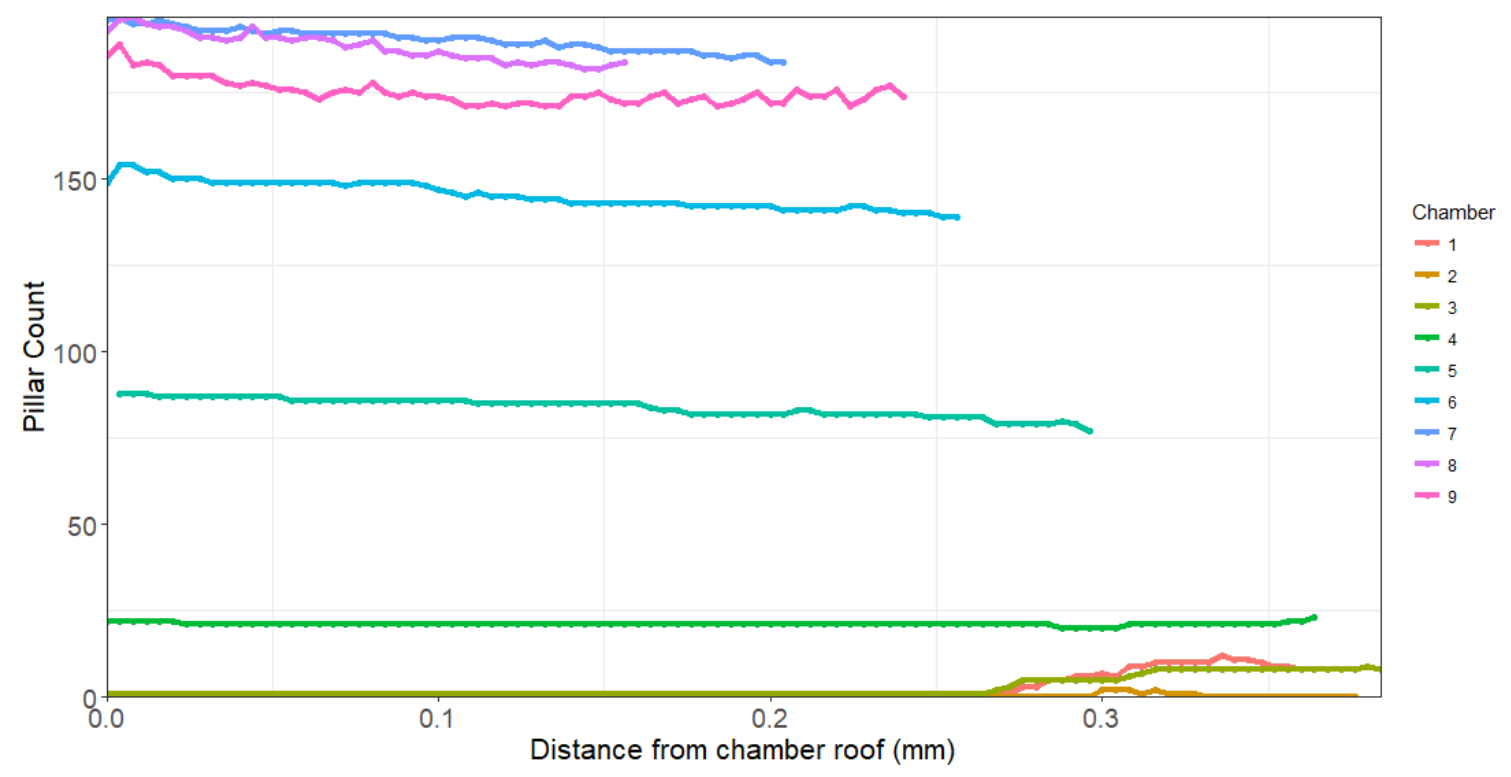

Figure 5.19: Pillar counts recorded by distance from chamber roof for each chamber in core_1b.

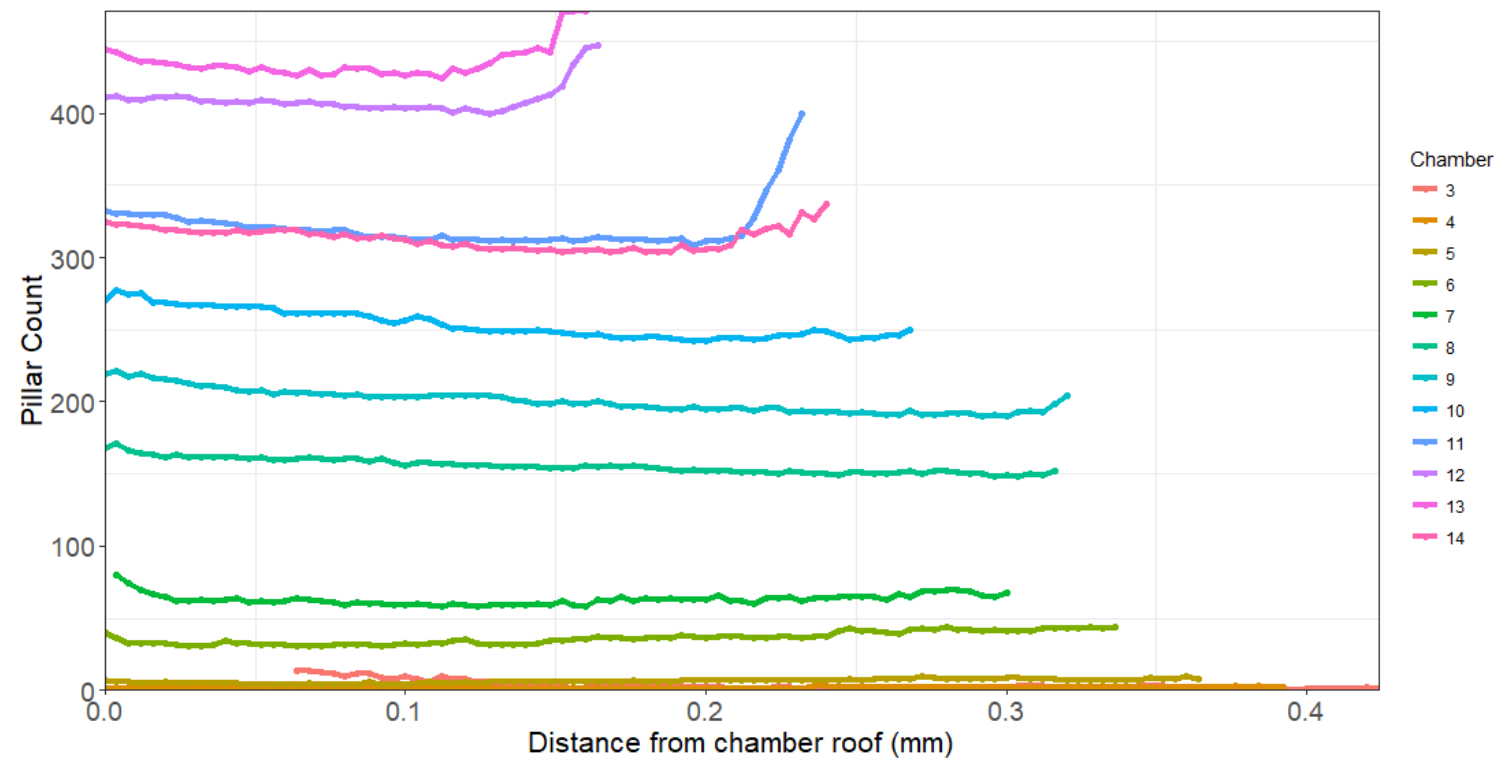

Figure 5.20: Pillar counts recorded by distance from chamber roof for each chamber in core_2a. 


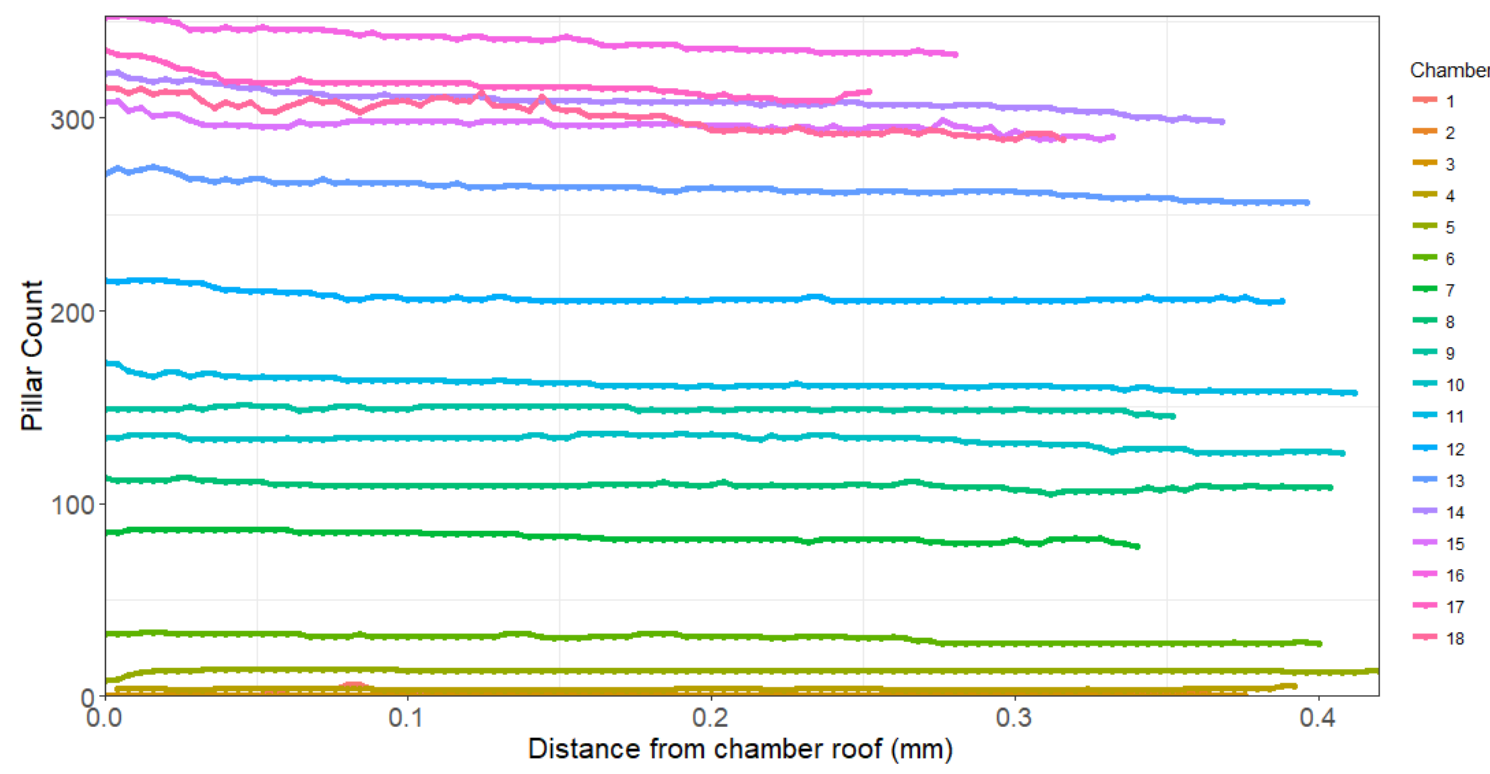

Figure 5.21: Pillar counts recorded by distance from chamber roof for each chamber in core_2b.

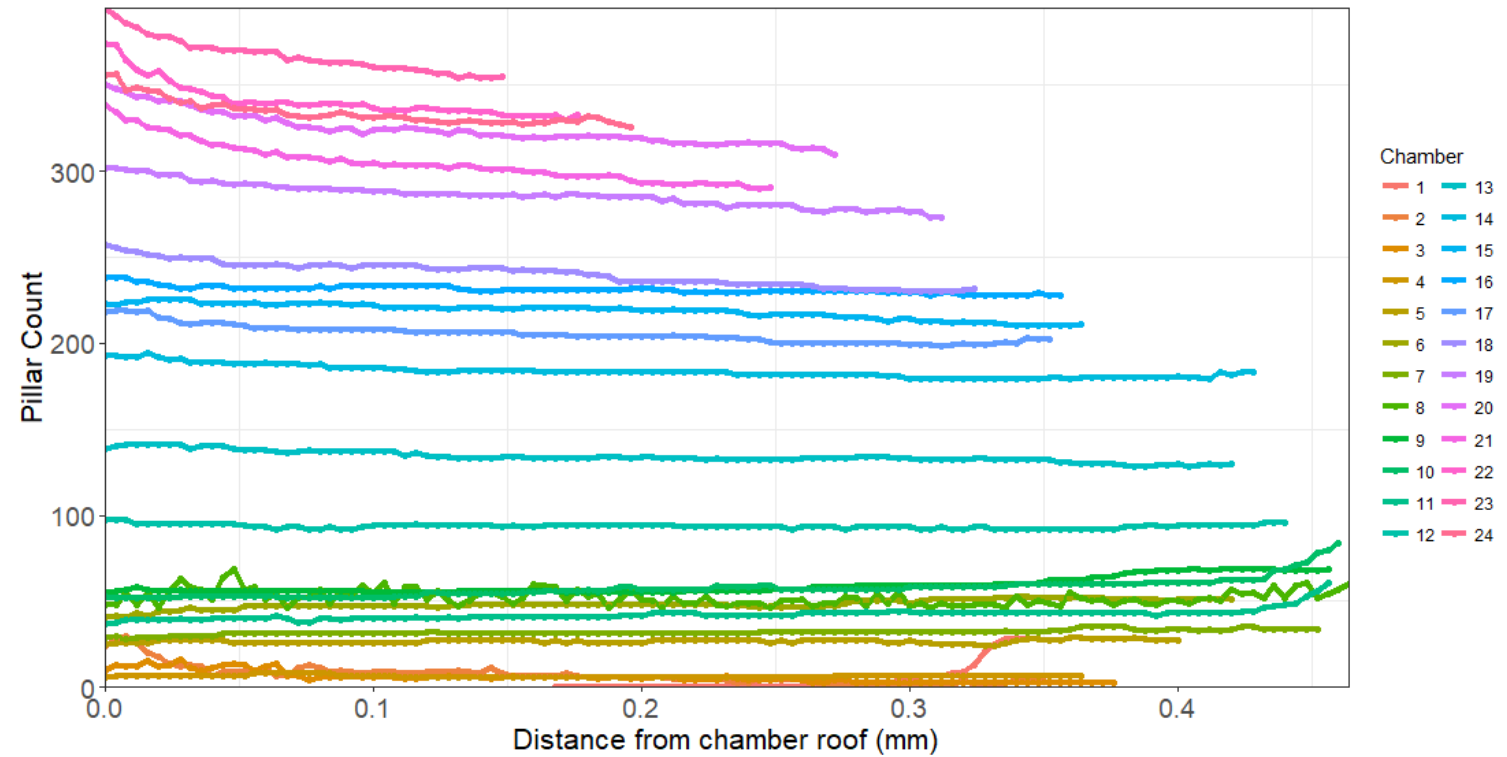

Figure 5.22: Pillar counts recorded by distance from chamber roof for each chamber in core_3c. 


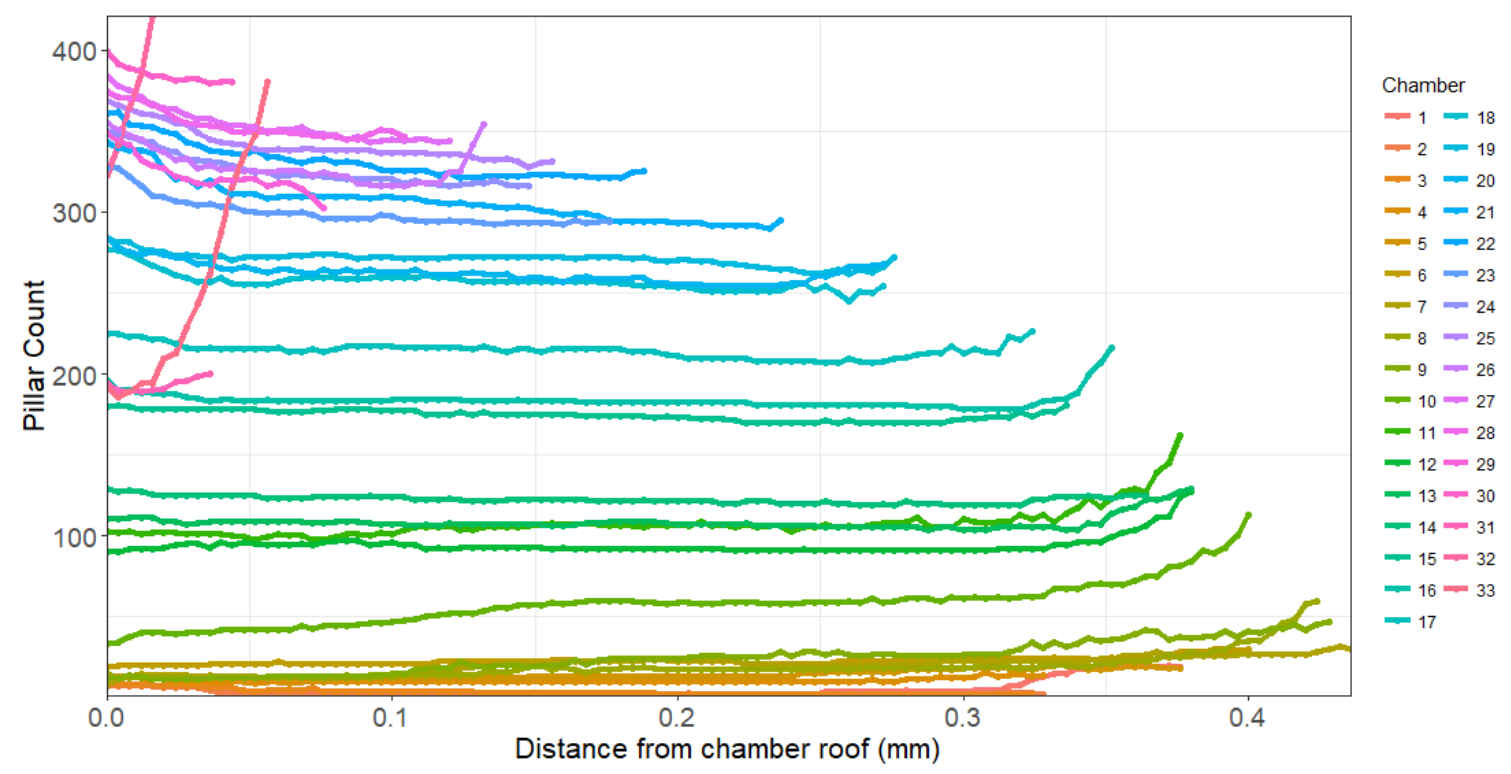

Figure 5.23: Pillar counts recorded by distance from chamber roof for each chamber in core_4c.

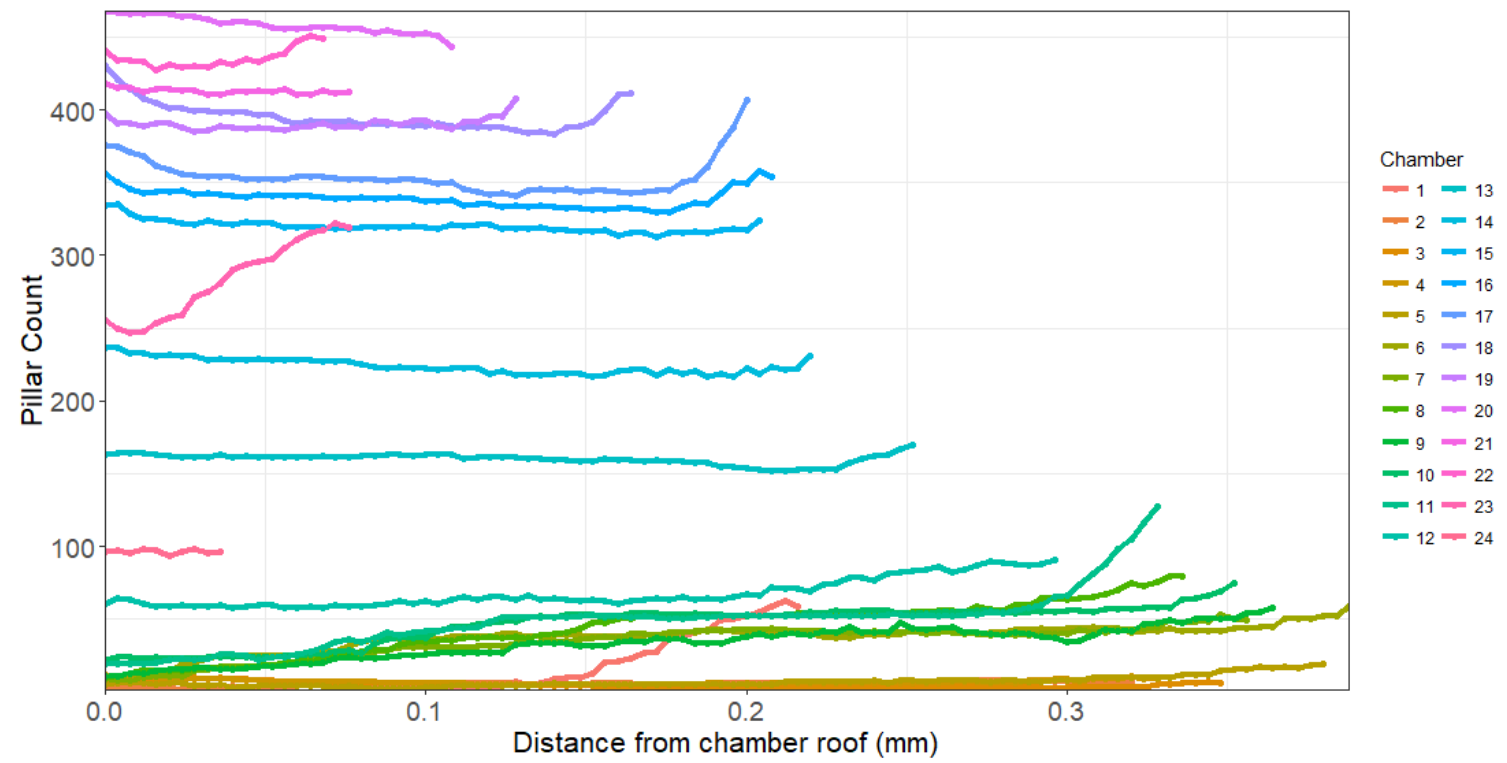

Figure 5.24: Pillar counts recorded by distance from chamber roof for each chamber in core_5c. 


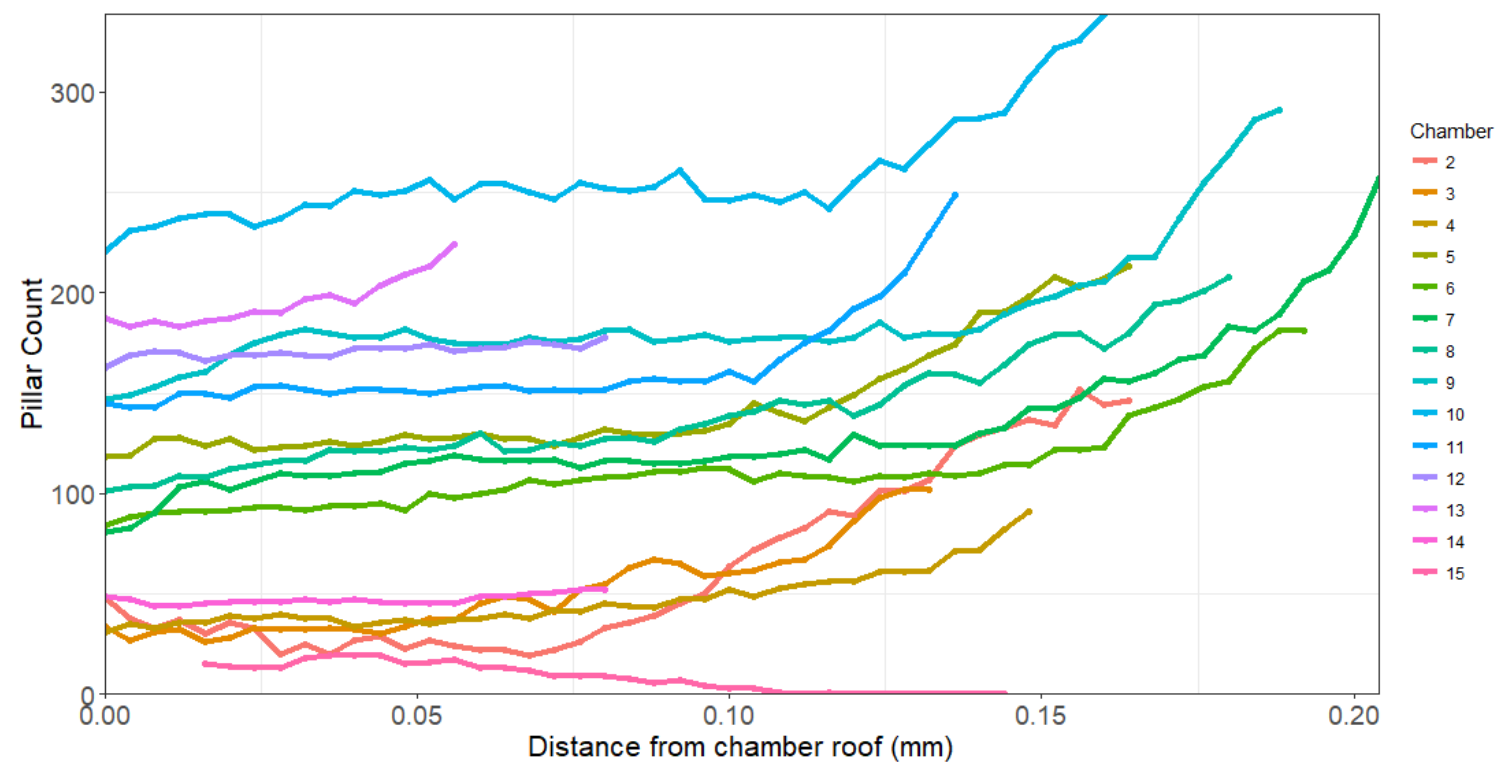

Figure 5.25: Pillar counts recorded by distance from chamber roof for each chamber in core_6b.

The pillar counts in core_1b (Figure 5.19) are generally consistent throughout each chamber, with some chambers even showing slight decreasing pillar counts towards the venter contrary to Example 5.3.1.1. Chambers 1, 2 and 3 (those in proximity to the dorsal shield) have very few, if any, pillars. This is mainly due to pillars having to be removed from analysis for reasons stated in section 5.2.2.2, however these pillars are considered qualitatively in the discussion. The sampling areas only capture sections of pillars, the characteristics of these pillars being long length and low tortuosity. Pillar counts are far higher in the ventral chambers, with close to 200 pillars in each of the sampling areas in chambers 7, 8 and 9 .

Pillars counts in core_2b and core_3c show a similar pattern to core_1b in that the pillar counts are very consistent and slightly decrease in some cases. These cores have far greater pillar counts near the ventral edge (between 300 - 400 in each chamber sampling area), this can be explained by the individual pillar size, which reduces dramatically in these areas of the cuttlebone.

Core_2a shows some interesting behaviour towards the floor of the ventral chambers. There is a sharp increase of pillar counts in chambers 11, 12, 13 and 14, but particularly in chamber 11. This could be due to some pillars 'splitting' at the bottom of the chamber. It is also worth noting that chambers 12 and 13 contain some of the highest pillar counts of any of the sampling areas, with only some chambers in core_5c showing higher counts.

Cores $4 \mathrm{c}, 5 \mathrm{c}$ and $6 \mathrm{~b}$ are taken towards the posterior of the cuttlebone and are all found in the siphuncular region. Nearly all chambers across the three cores show a consistent trend of an increasing pillar count 'tail' towards the chamber floor. These tails are particularly evident in core_6b, with a more gradual increase in each chamber than anywhere else on the 
cuttlebone.

Chambers 32 and 33 in core_4c and chamber 23 in core_5c show sharp increases in pillar count across their limited number of slices. Table 5.8 shows that chamber 33 in core_4c and chamber 23 in core_5c are only partially captured in cores and consequentially it is hard to infer anything from this. Figure 5.26 shows slices 00 and 13 of chamber 32 of core_4c. It is evident from these slices that the count is increased, as the pillars seemingly become arranged into short, straight lines with their mean spacing drastically reduced. The thick white bands on the left and right hand sides of (b) are the septum floor with the top of the chamber below seen slightly on these edges.

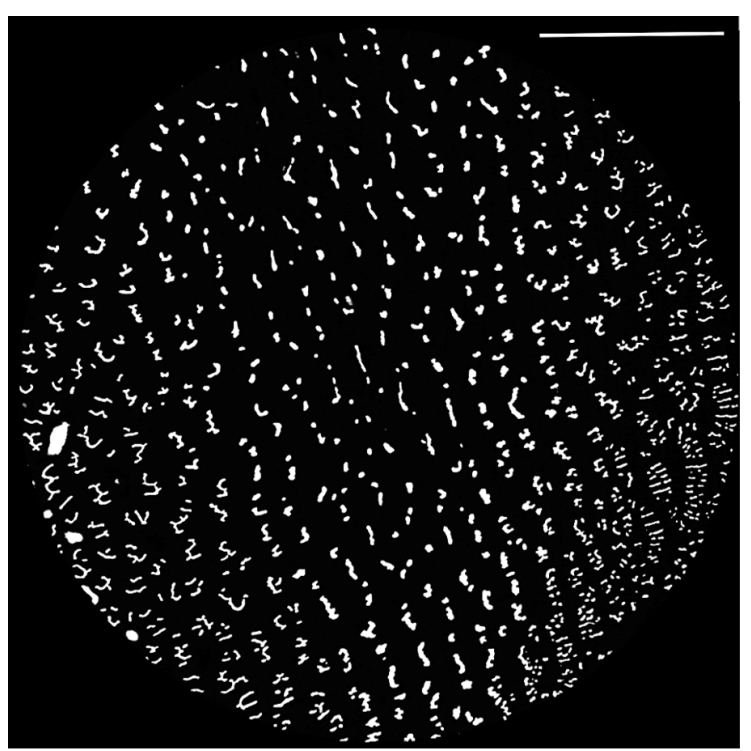

(a)

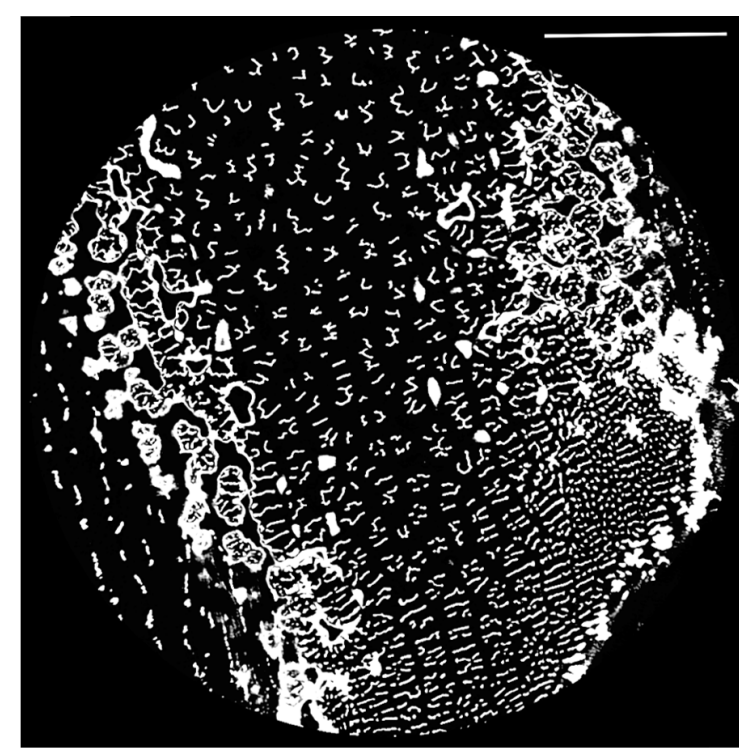

(b)

Figure 5.26: Sliced CT data of core_4c - chamber 32; (a) slice 00, (b) slice 13. Scale bar = $0.5 \mathrm{~mm}$.

Core_6b, perhaps due to having fewer chambers, has far lower pillar counts than core_4c or core_5c.

Another aspect of pillar count analysis is how the pillars relate between adjacent chambers. All the pillar count figures show that adjacent chambers can often have very different pillar counts. There is a general trend that chamber pillar counts increase towards the ventral of the cuttlebone, however this is not a strict trend, as the most ventral chambers in each core often show.

Checa et al. [184] noted that pillars appear to grow in consistent positions in successive chambers, however varying pillar counts between chambers suggest that this may not be the case. Figure 5.27 displays two examples from core_4c of a slice at the floor of a chamber (black) overlaid on a slice from the roof of the succeeding chamber (red). Both images show instances of some close alignment between certain pillars, and also many clearly misaligned 
pillars. Image (b) appears to have more pillar alignment, however it is hard to visually judge if there is an underlying trend. An appropriate measure of how consistent pillar positions are across chambers would need to be developed. One possible method could be to compare the material-to-air percentage for the combined slices against the material-to-air percentage of the ceiling slice. A low percentage difference, relatively, corresponds to higher pillar alignment (a difference of zero would indicate perfect pillar alignment).
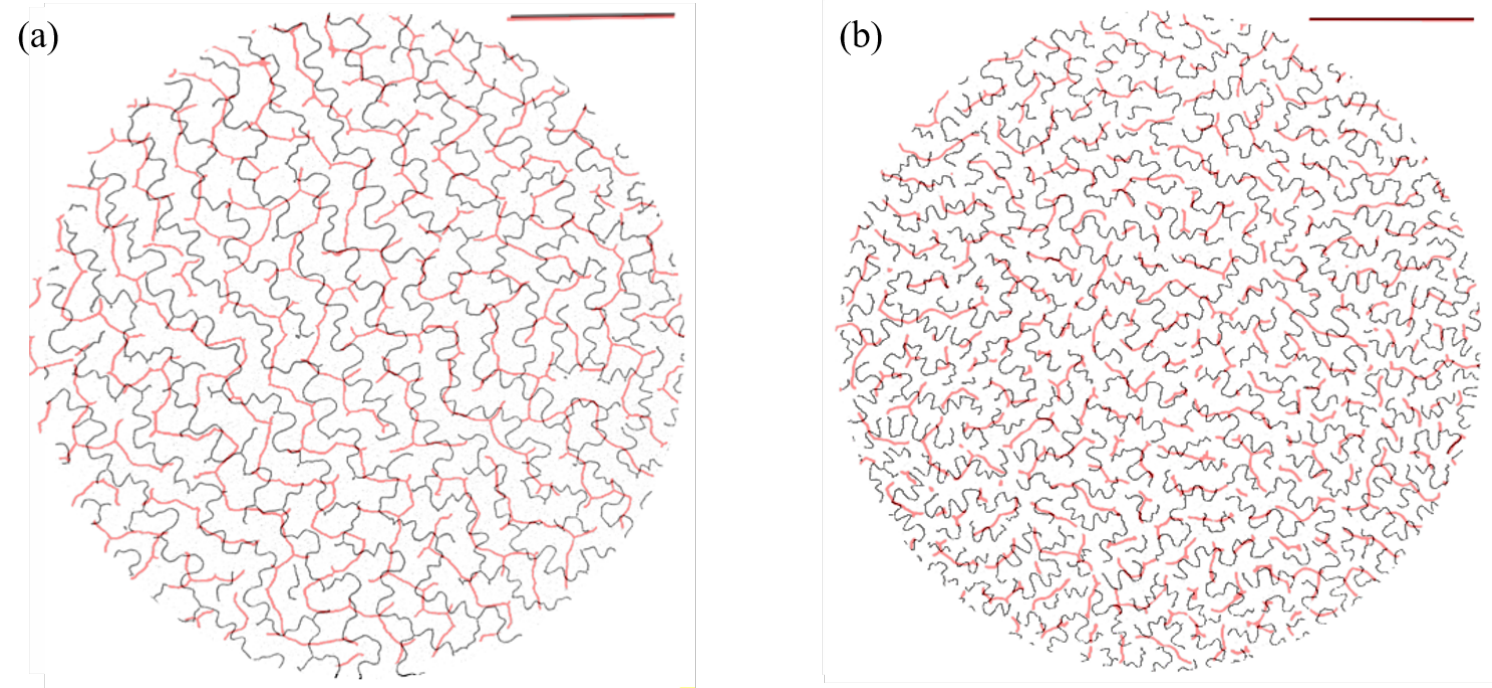

Figure 5.27: Overlaid slices taken from core_4c showing pillar alignment. (a) black - chamber 3 - slice 86, red - chamber 4 - slice 0, (b) black - chamber 14 - slice 91, red - chamber 15 slice 0 .

Material-to-air percentage The material-to-air percentage was calculated by selecting a region within a binarised slice and calculating the percentage of material (material flag $=$ 1) to air (material flag $=0$ ). Table 5.9 in Example 5.3.1.1 shows that the material-to-air percentage decreases and increases by small amounts through the chamber.

Figures 5.28-5.34 display the material-to-air percentages recorded on each slice for each chamber in each sample core.

All cores display flat, or decreasing (particularly cores 3c, 4c and 5c), material percentages in the first few slices from the roof of the chambers. Then material percentages begin to rise, gradually at first, but then almost exponentially towards the floor of the chambers. The only core not to follow this trend is core_6c, where the increase in material-to-air percentage remains generally linear, even in the final slices of the chambers. Only chambers 14 and 15 show a rise similar to those in other cores, but as mentioned previously, these chambers are only partially represented.

Larger chambers, i.e. those with the most slices, appear to have higher initial materialto-air percentages than small chambers. For example, chamber 9 in core_3c has a materialto-air percentage of $14.1 \%$, whereas chamber 23 close to the ventral edge has a material 


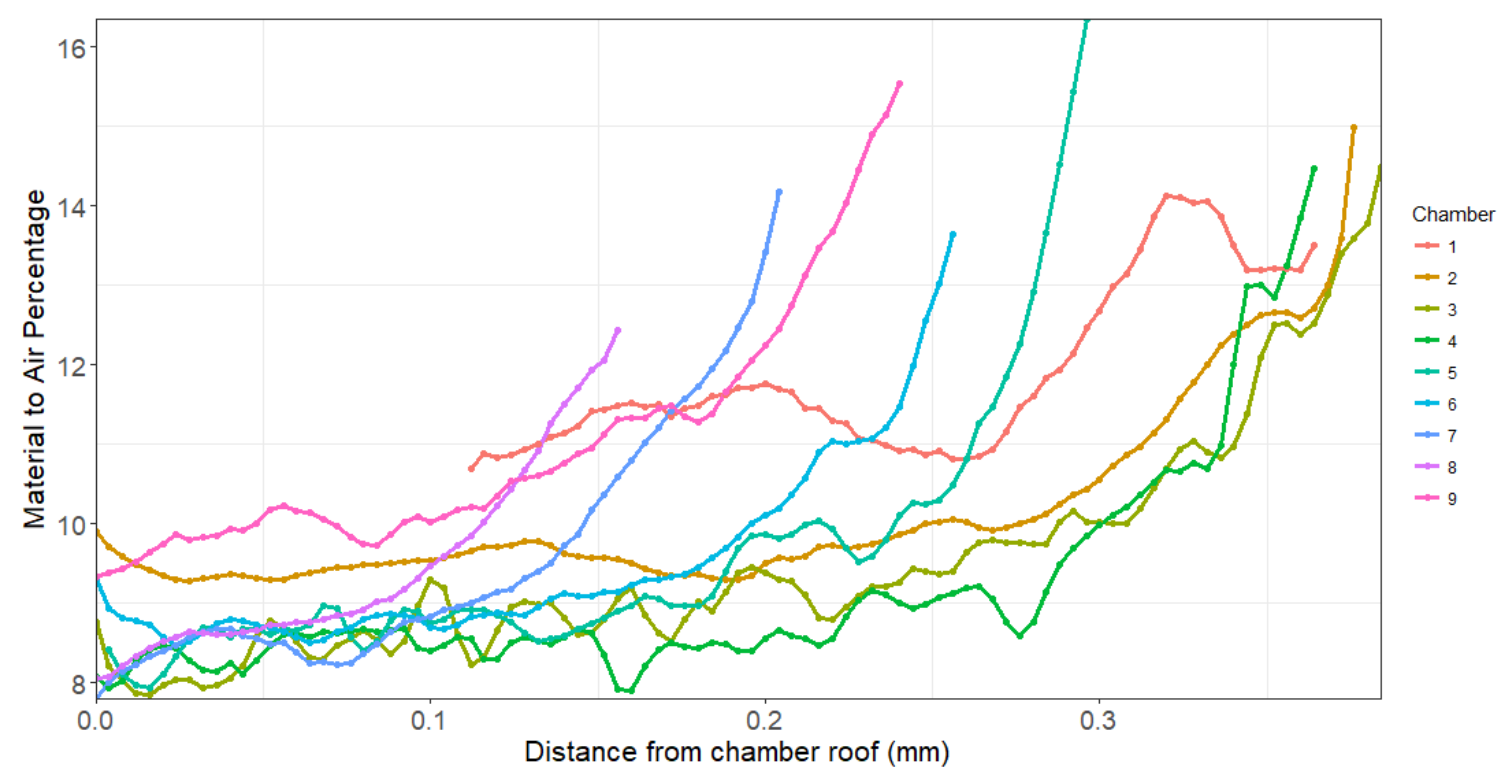

Figure 5.28: Material-to-air percentage recorded by distance from chamber roof for each chamber in core_1b.

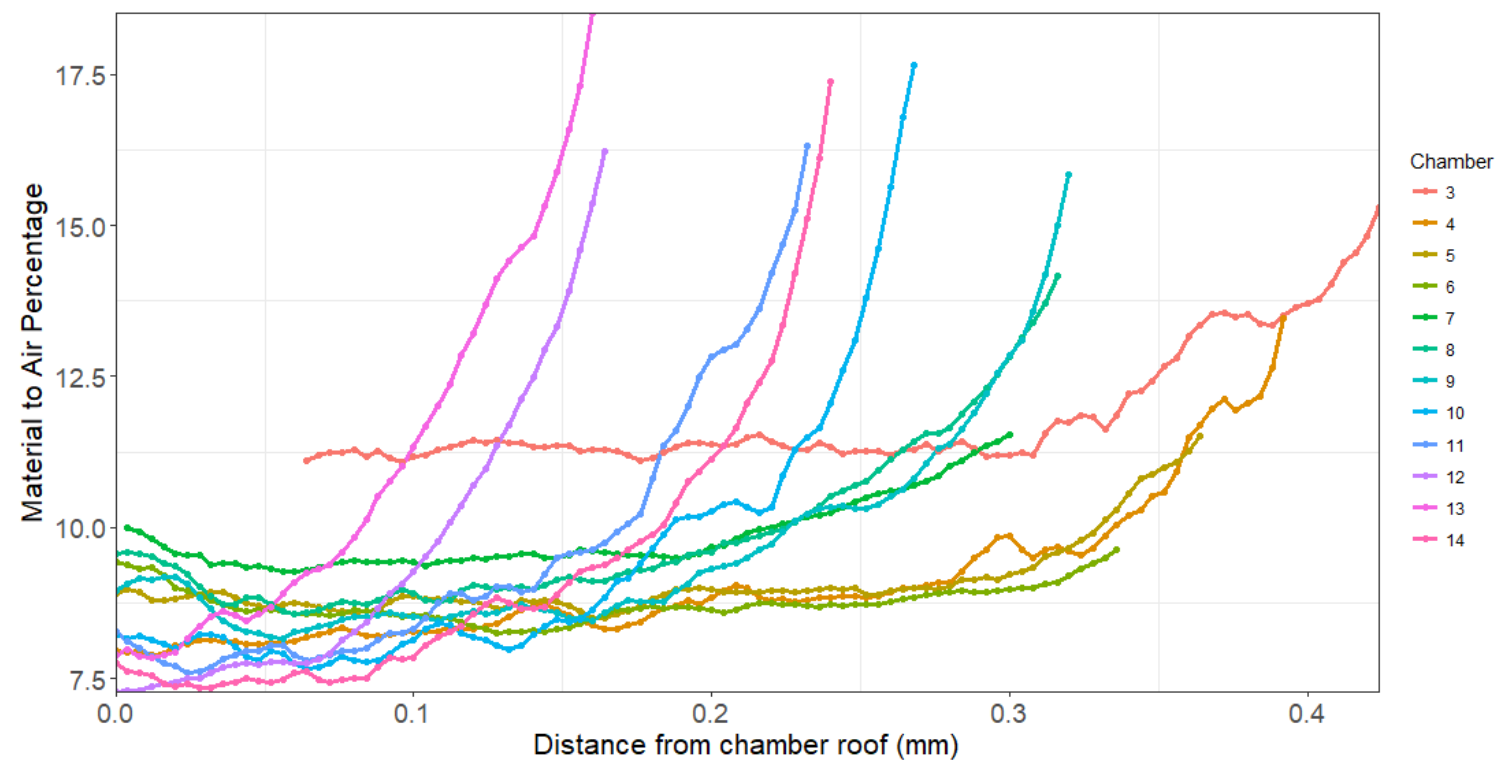

Figure 5.29: Material-to-air percentage recorded by distance from chamber roof for each chamber in core_2a. 


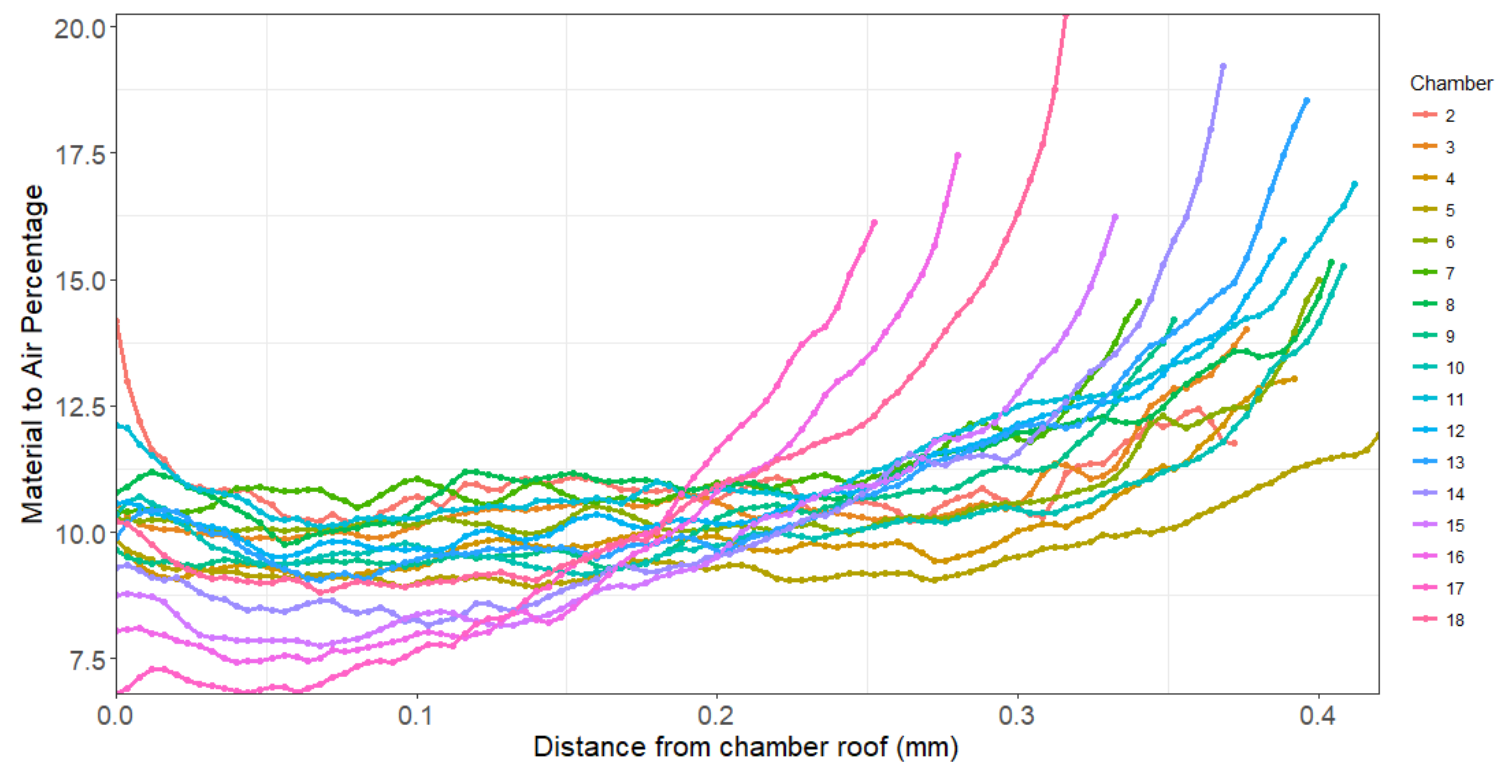

Figure 5.30: Material-to-air percentage recorded by distance from chamber roof for each chamber in core_2b.

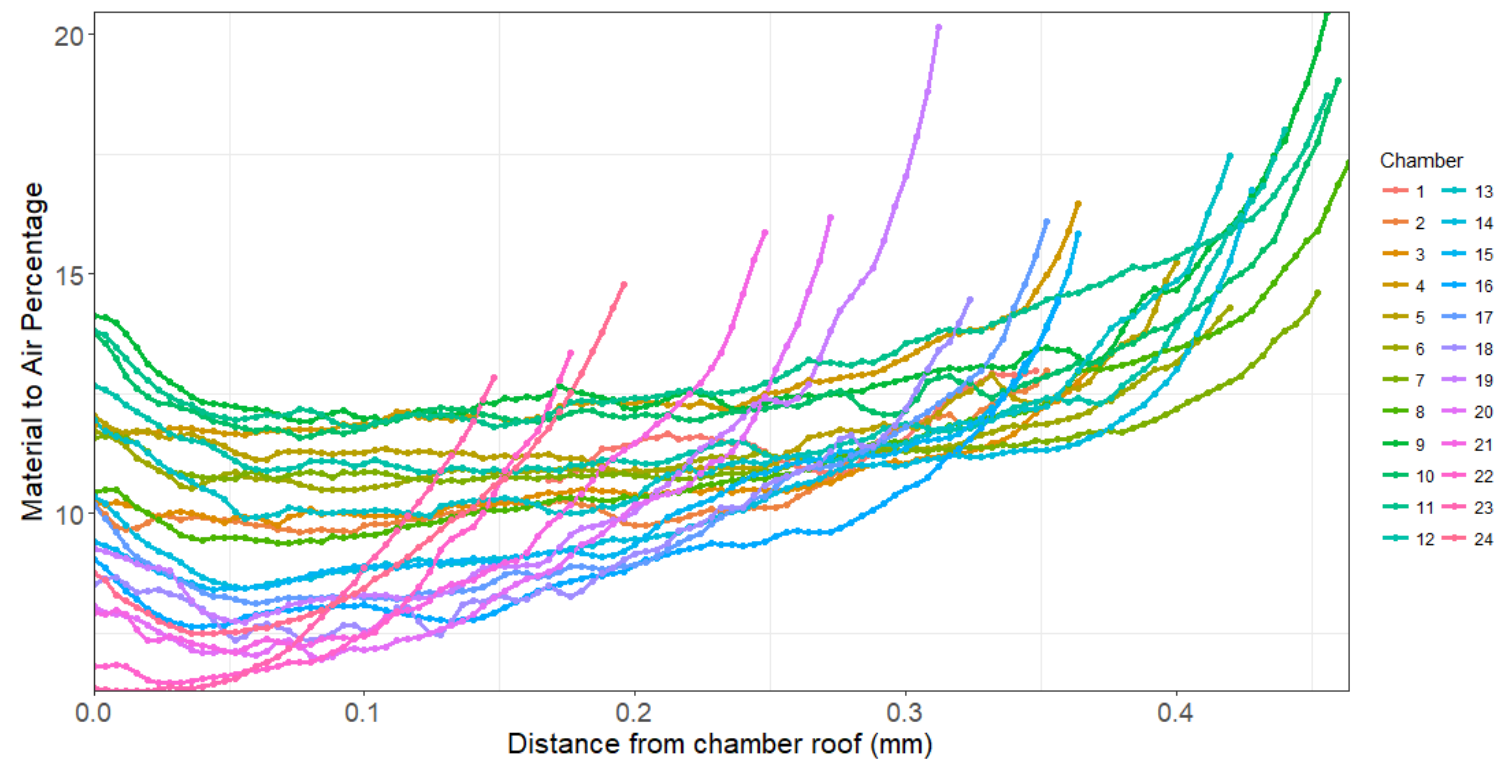

Figure 5.31: Material-to-air percentage recorded by distance from chamber roof for each chamber in core_3c. 


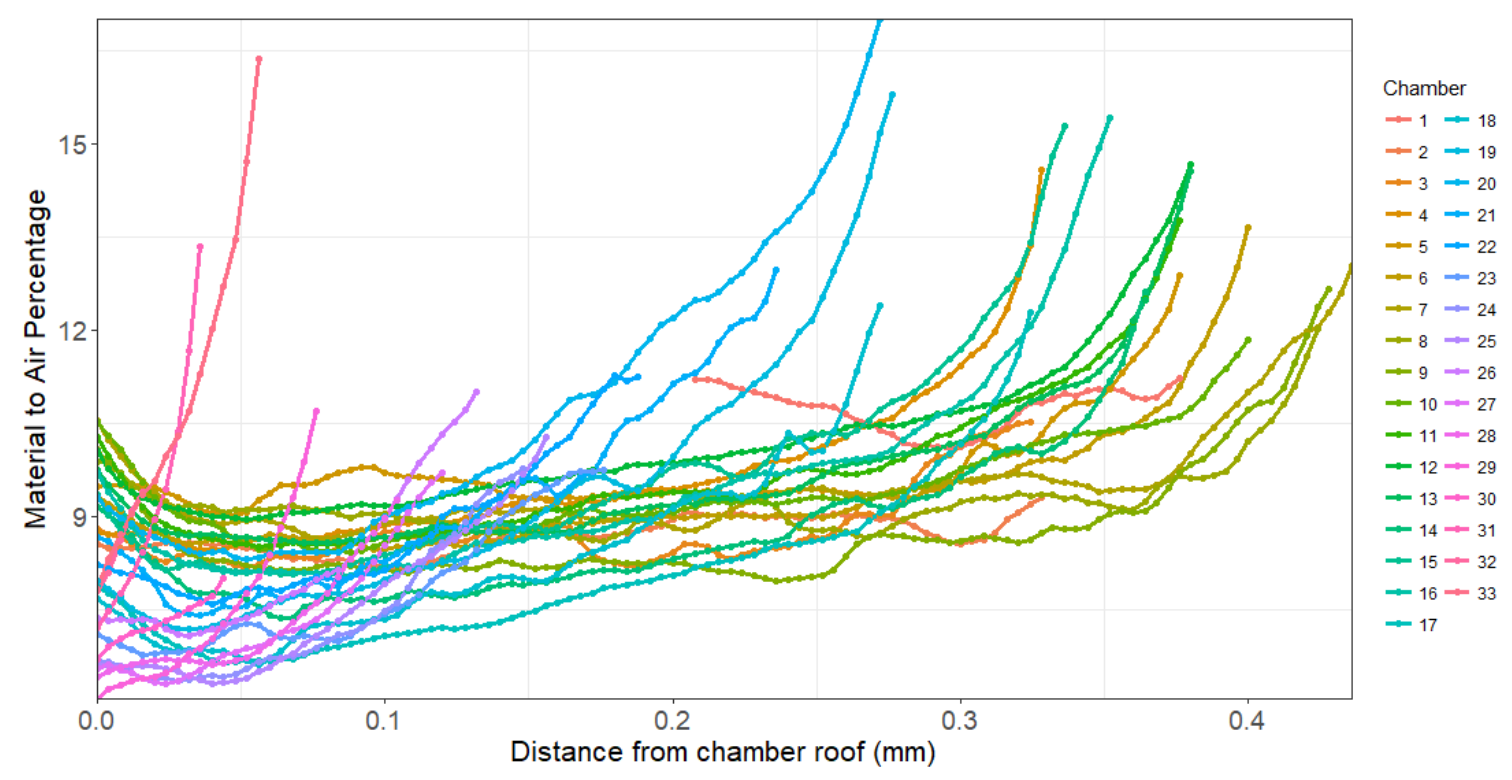

Figure 5.32: Material-to-air percentage recorded by distance from chamber roof for each chamber in core_4c.

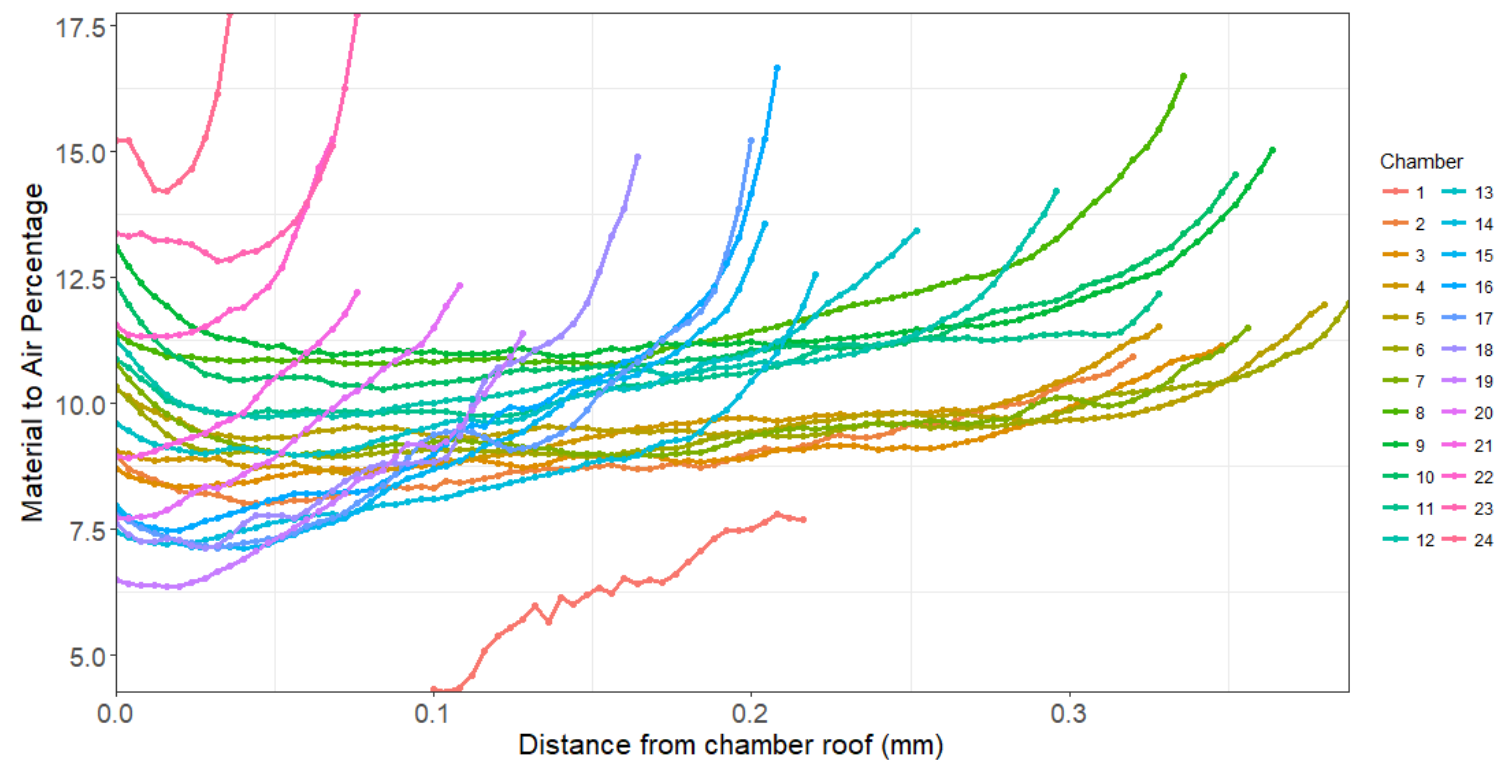

Figure 5.33: Material-to-air percentage recorded by distance from chamber roof for each chamber in core_5c. 


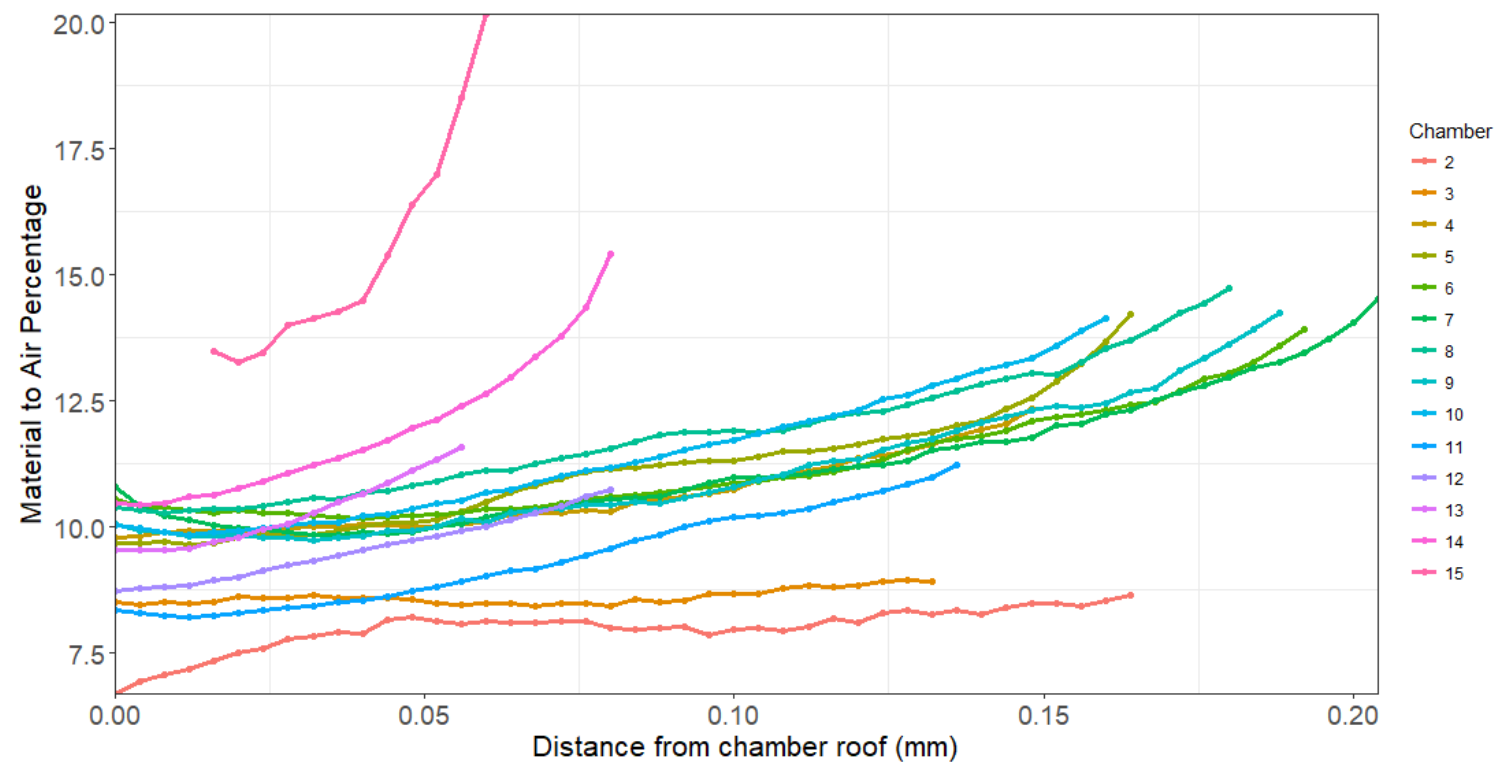

Figure 5.34: Material-to-air percentage recorded by distance from chamber roof for each chamber in core_6b.

percentage of just $6.4 \%$. The average increase in material-to-air percentage of a pillar from roof to floor is $4.5 \%$, however it can be as high as $10.9 \%$.

Tortuosity Tortuosity $(\tau)$, in this case, is defined as the amount a pillar deviates from a straight line, with $\tau=1$ representing a straight line and $\tau \rightarrow \infty$ a loop. Pillar tortuosity is derived from the perimeter and Feret length of a pillar.

Table 5.9 in Example 5.3.1.1 shows that tortuosity first decreases slightly before increasing to a maximum of 1.8155 in slice 100. Figures 5.35 and 5.36 show the spread of individual pillar perimeter and Feret lengths for the slices given in Example 5.3.1.1 respectively. In the figures we can see that the median and interquartile range of perimeters across the slices remain generally consistent, and Feret length median and interquartile range decrease and reduce slightly respectively towards the ventral part of the chamber. Figure 5.37 shows the resulting spread of tortuosity calculated using equation (5.2.2.1). It is clear that, after an initial decrease, due to large outliers and lower pillar counts, the pillars increase consistently in tortuosity towards the ventral of the chamber. It is easy to see this visually in Figure 5.18, and since the images correspond with the tortuosity values, this also reinforces the assumption that equation (5.2.2.1) is an appropriate measure for the behaviour of the pillars.

Following on from this detailed example, Figures 5.38-5.44 display the average tortuosity calculated on each slice for each chamber in each sample core.

In core_1b (Figure 5.38), chambers 1, 2 and 3 show sudden changes in average tortuosity across the slices. This is due to low and irregular pillar counts. The large dip in chamber 


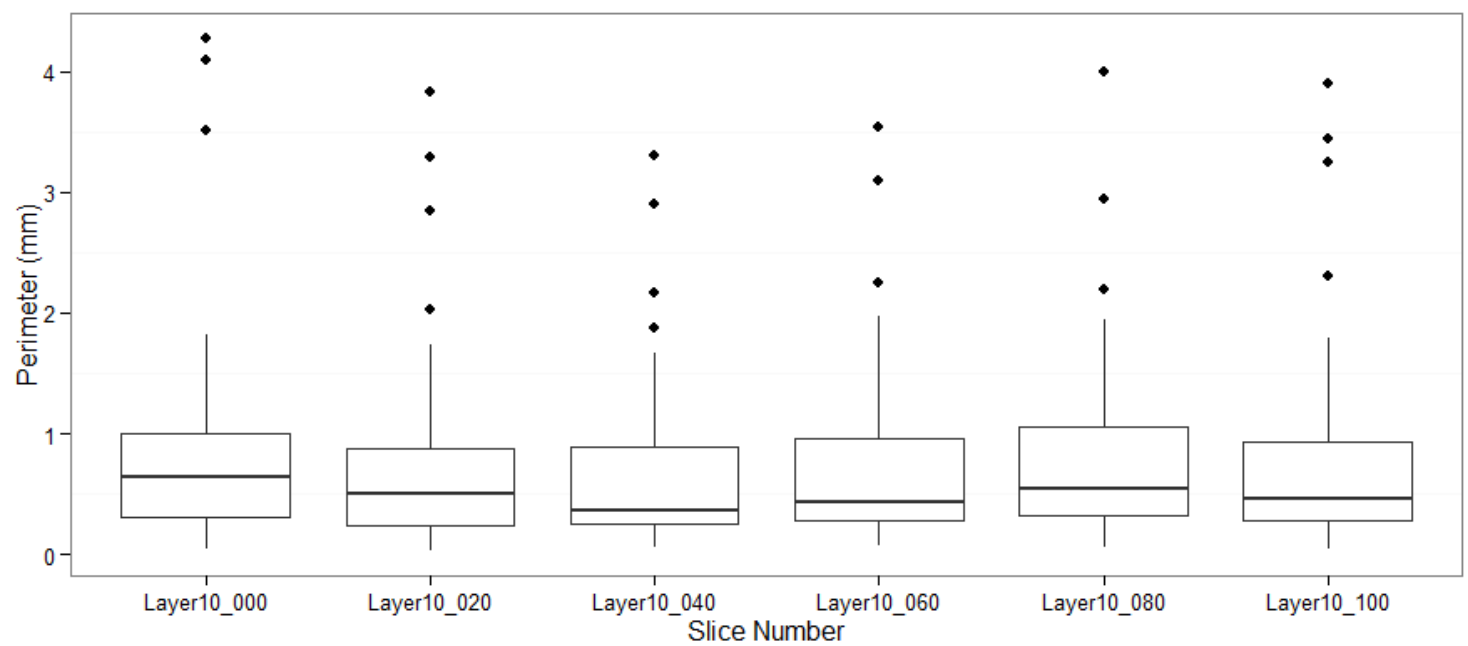

Figure 5.35: A boxplot showing the distribution of pillar perimeters for six slices taken from chamber 10 of core 4 .

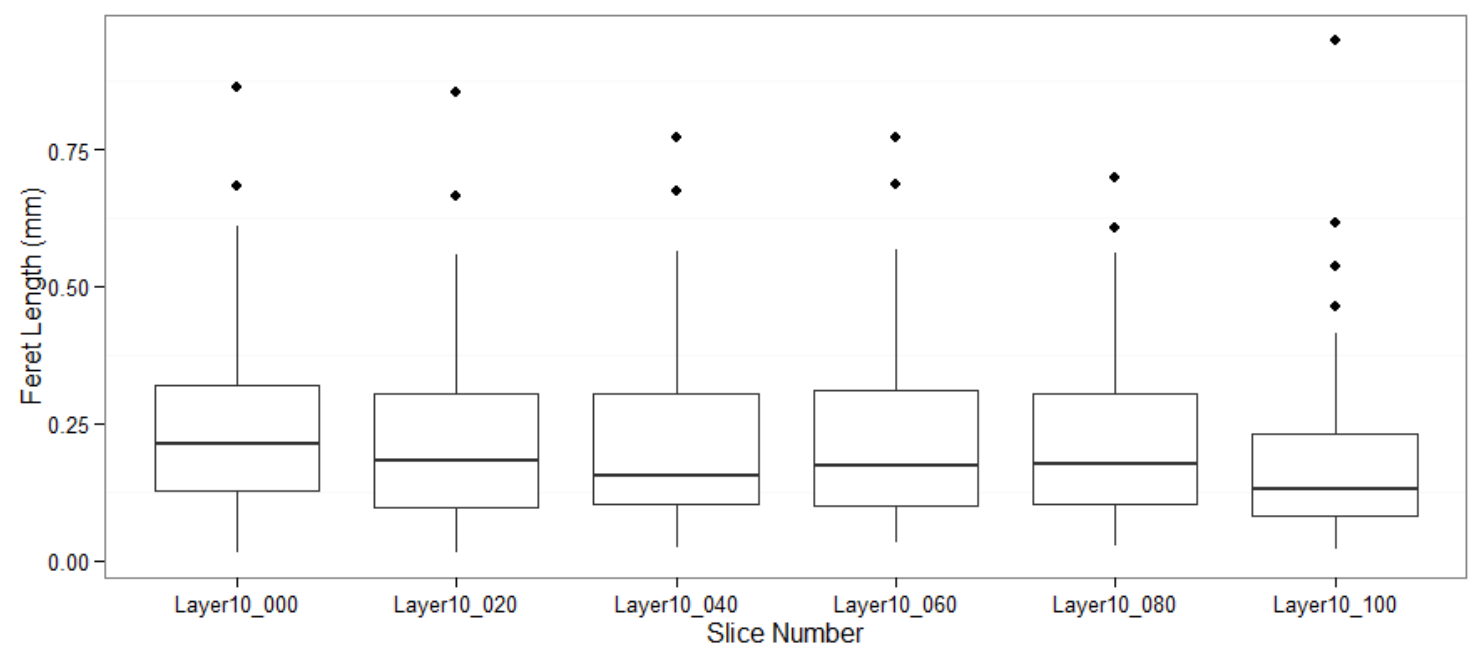

Figure 5.36: A boxplot showing the distribution of pillar Feret lengths for six slices taken from chamber 10 of core 4 . 


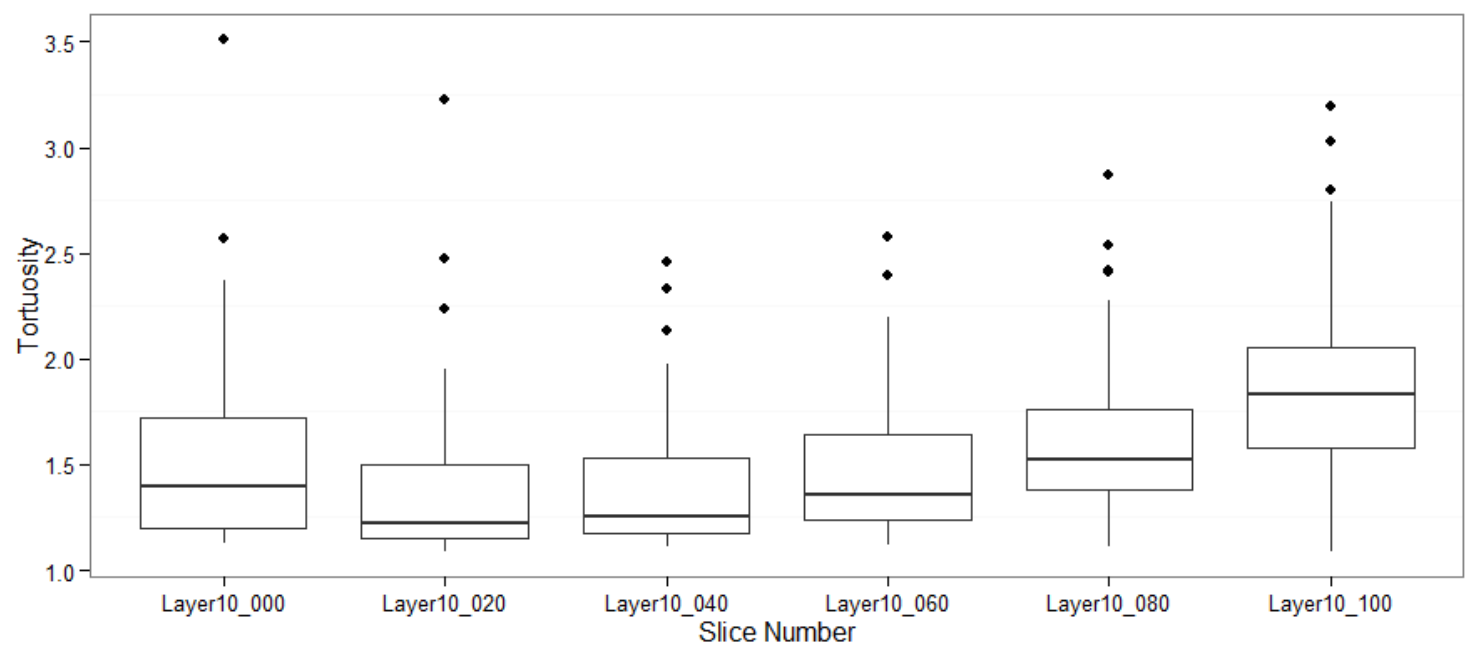

Figure 5.37: A boxplot showing the distribution of pillar tortuosity for six slices taken from chamber 10 of core 4 .

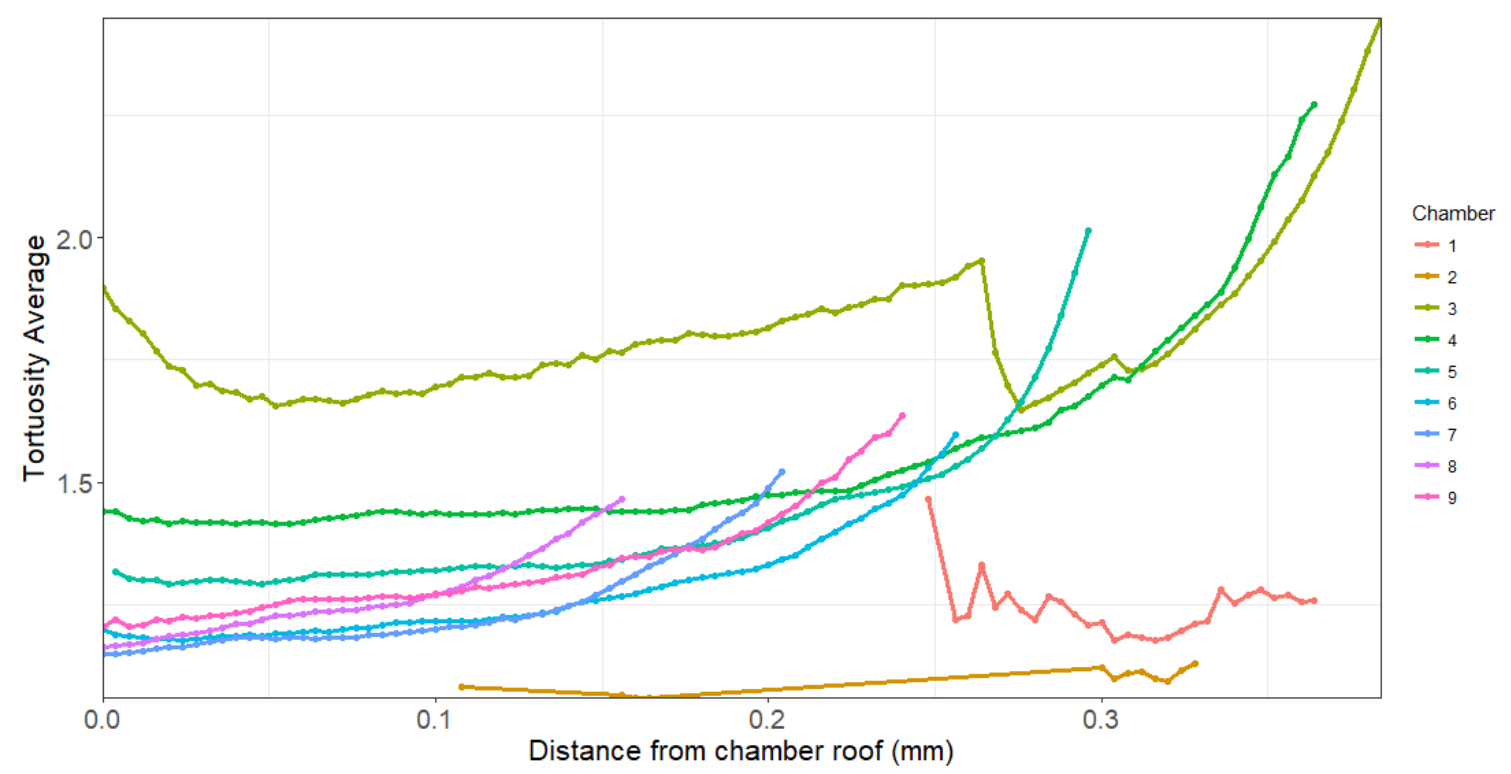

Figure 5.38: Average tortuosity recorded by distance from chamber roof for each chamber in core_1b. 


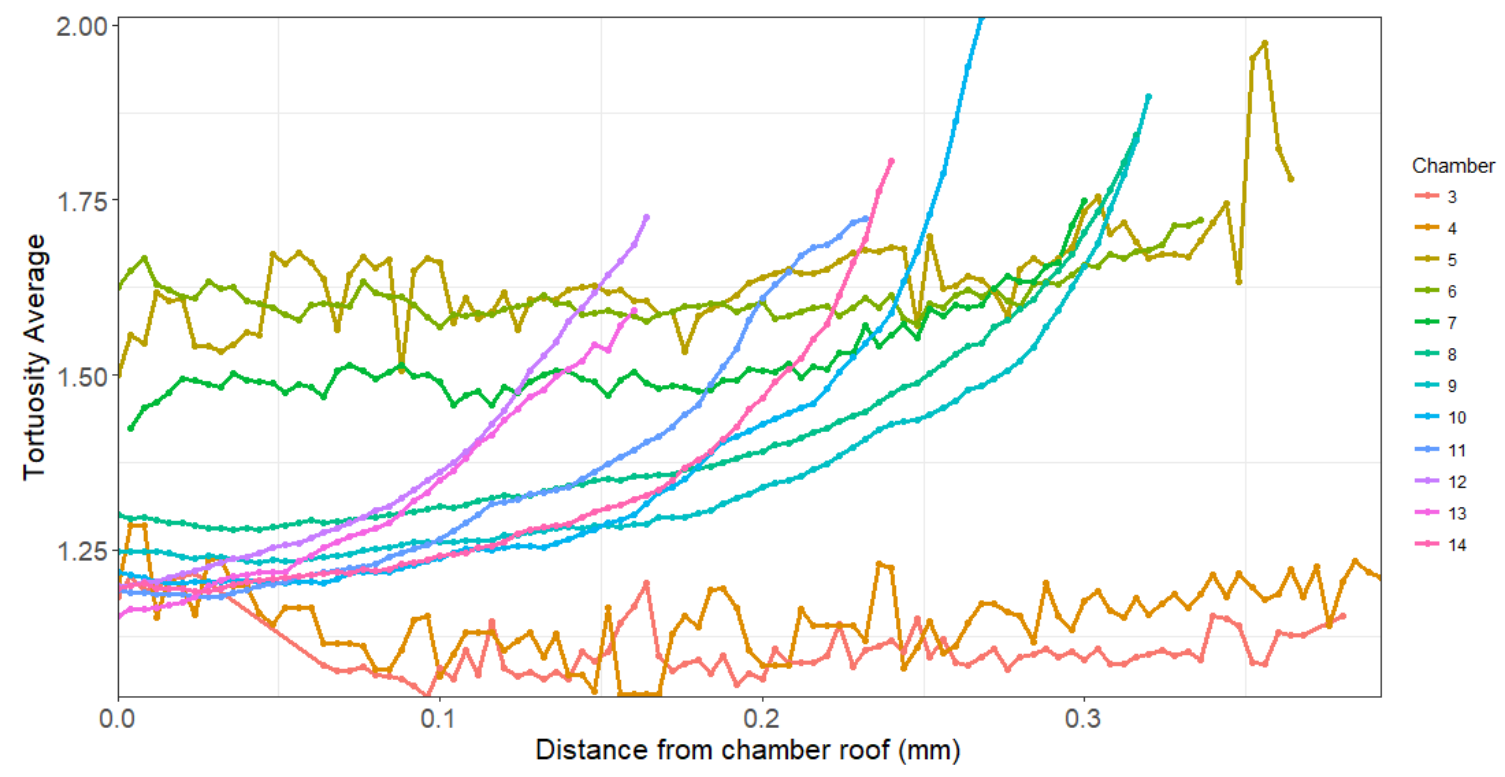

Figure 5.39: Average tortuosity recorded by distance from chamber roof for each chamber in core_2a.

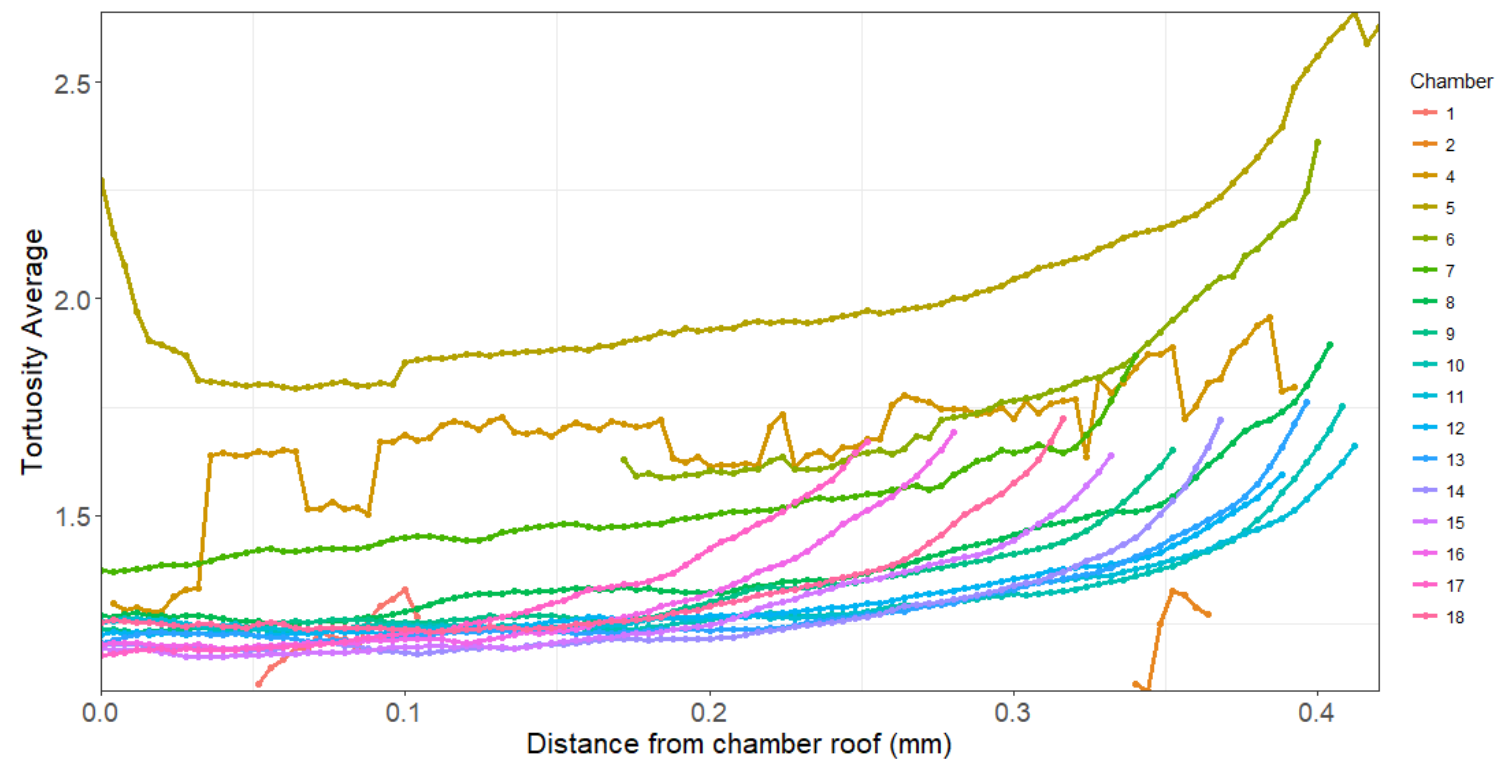

Figure 5.40: Average tortuosity recorded by distance from chamber roof for each chamber in core_2b. 


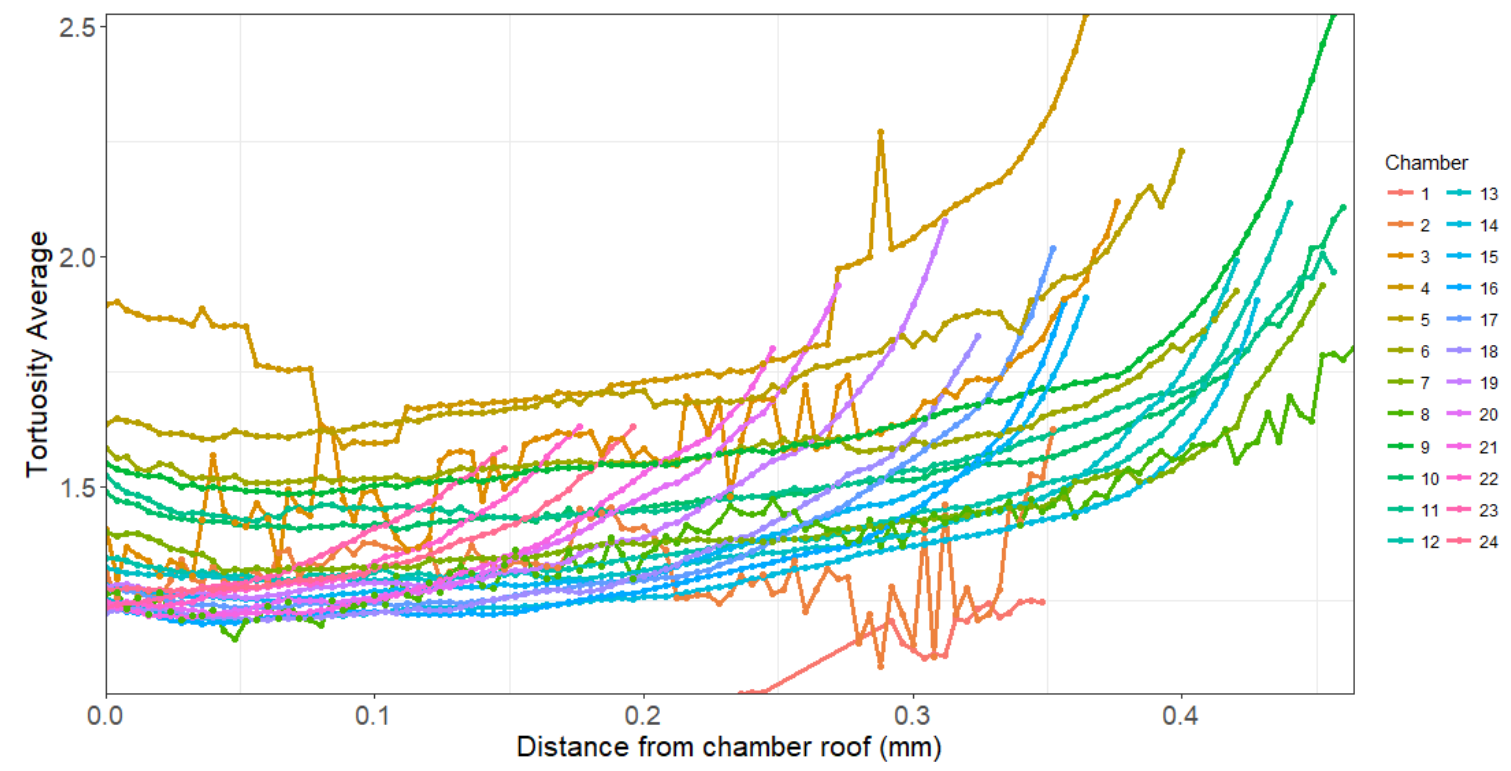

Figure 5.41: Average tortuosity recorded by distance from chamber roof for each chamber in core_3c.

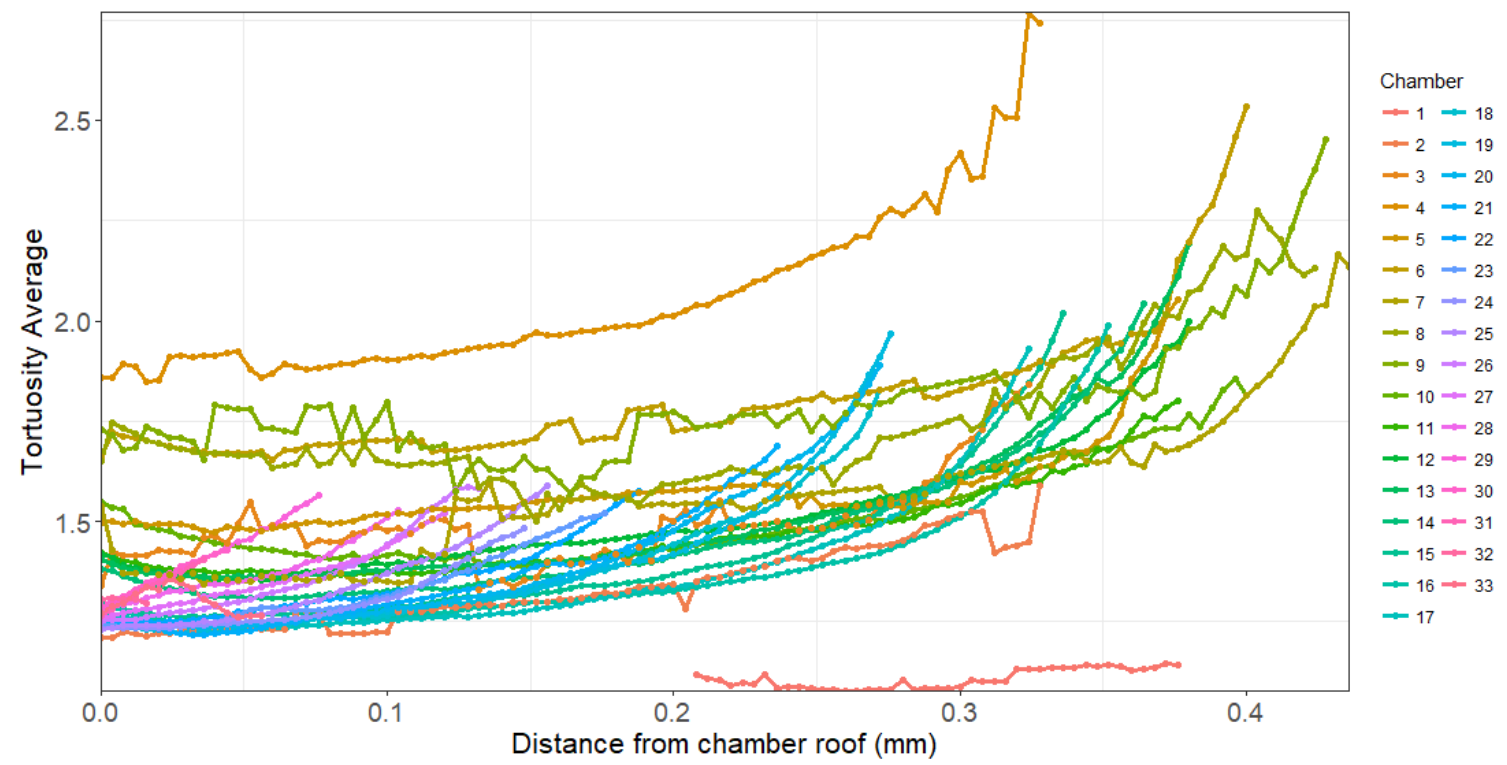

Figure 5.42: Average tortuosity recorded by distance from chamber roof for each chamber in core_4c. 


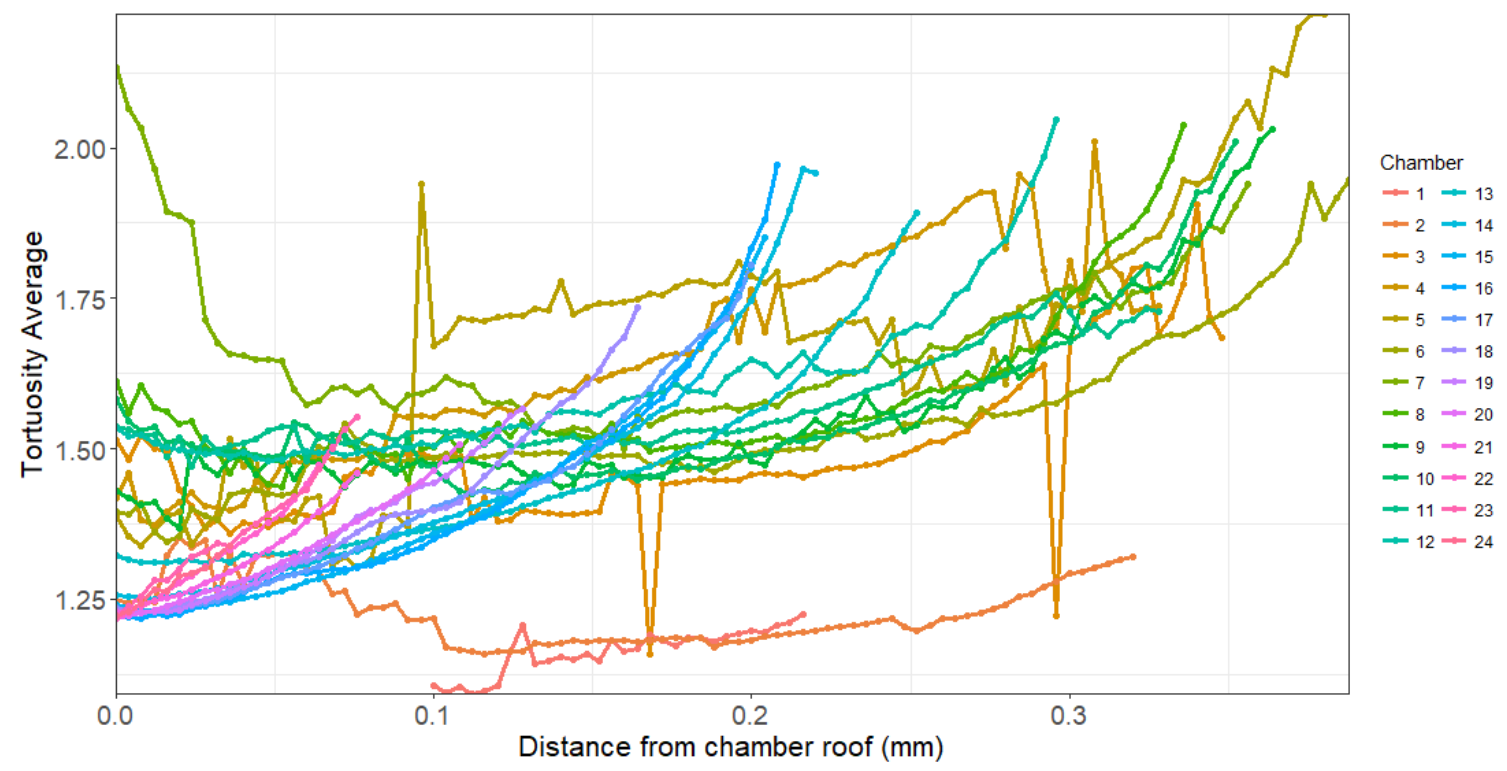

Figure 5.43: Average tortuosity recorded by distance from chamber roof for each chamber in core_5c.

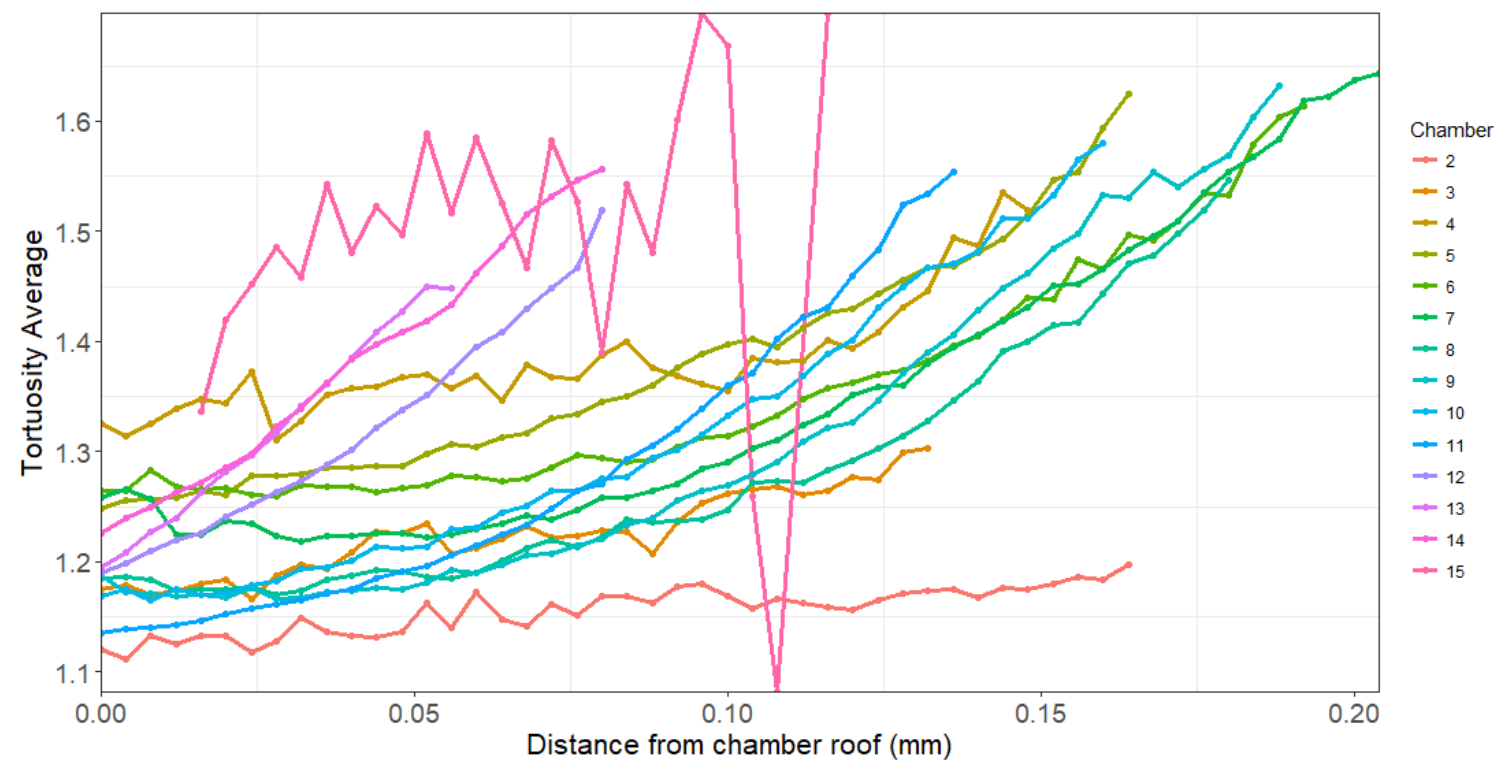

Figure 5.44: Average tortuosity recorded by distance from chamber roof for each chamber in core_6b. 
3 , for example, is attributed to only one pillar being considered until slice 67 , where at this point, more pillars are counted and so the average changes. Figure 5.45 shows four slices in chamber 3, pillars which are usable in each slice are coloured red. Between slices 60 and 80, the usable pillar count increases from one to eight. It is clear that most pillars in these slices are not included in the quantitative analysis since they are in contact with the edge of the sampling area. Pillars of this nature and in these areas of the cuttlebone are instead considered qualitatively later in the discussion. Chambers 4 - 9 show a similar trend to Example 5.3.1.1 where tortuosity increases towards the ventral of the chamber. Higher pillar counts in these chambers contribute towards a smoother curve.

In core_2a (Figure 5.39), chambers 3 - 6 have low pillar counts, which cause the fluctuating tortuosity values. The rest of the chamber sampling areas have more pillars, and the trend becomes far smoother and similar to that seen in core_1b.

Similar trends are seen across the rest of the cores, with sudden changes in the tortuosity lines explained by either low pillar counts, or specific pillars being removed (or included) in analysis, usually as a result of them becoming in contact (or no longer being in contact) with the edge of the chamber sampling area. It is also noticeable that chambers towards the venter of cuttlebone have roughly equivalent tortuosity averages at both the first and last slices in a chamber, across the cores. The tortuosity values tend to be between 1.2 and 1.3 at the dorsum of the chamber and 1.6 to 1.9 for the venter of the chamber.

Core_6b again stands out from the other cores. The pillars in each chamber only show a modest increase in tortuosity through the chamber, unlike the exponential-like ramp-up of tortuosity seen towards the ventral area of chambers in all other cores (this is noticeable in core_3c, for example).

Framework for morphological features within cuttlebone Following on from the quantitative measurements obtained from each of the seven cores, schematic representations are suggested for pillar count, material-to-air percentage and tortuosity of the entire cuttlebone. Values for each measurement are grouped into large bands, and further work would aim to validate these general frameworks across a range of ages and sizes of cuttlebone. The middle slice of each chamber was used to guide the summaries. Regions where limited data was collected, or exhibited potential anomalies, were correlated qualitatively to ensure they conformed to the framework. The summary data with conditional formatting that informed the creation of these schematics are given in Figures 5.46 and 5.47.

Figure 5.48 displays pillar counts per $3.14 \mathrm{~mm}^{2}$ across the cuttlebone in the transverse and longitudinal directions. Pillar counts can be grouped into low (green; 0 - 100 pillars), medium (amber; 101 - 300 pillars) and high (red; 301 - 500 pillars) bands. The results across the sample cores were remarkably consistent; chambers near the dorsal shield had low pillar counts. There is then quite a sudden increase in pillar count through the middle chambers, indicated by the thin amber region on both schematics, rising to the high pillar counts in the most ventral chambers. This pillar count framework suggests that the siphonal zone is 


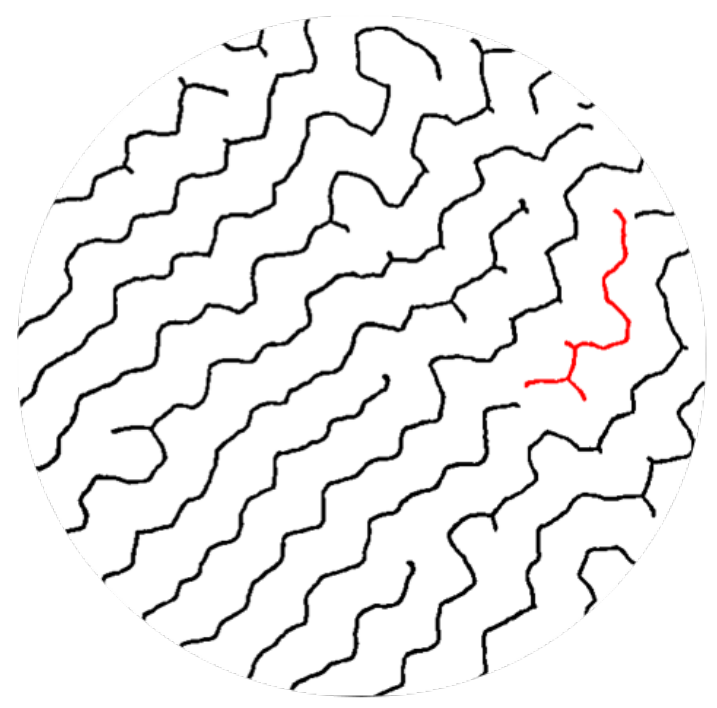

(a)

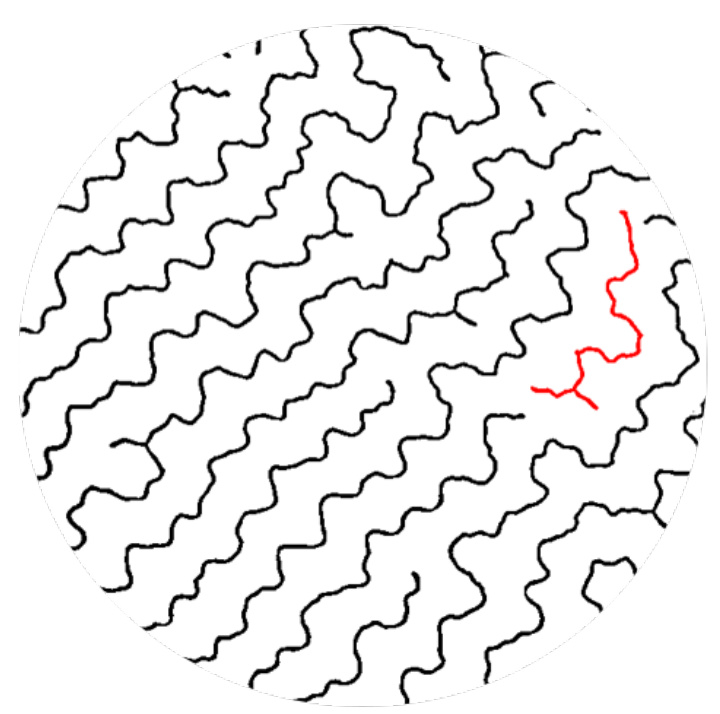

(c)

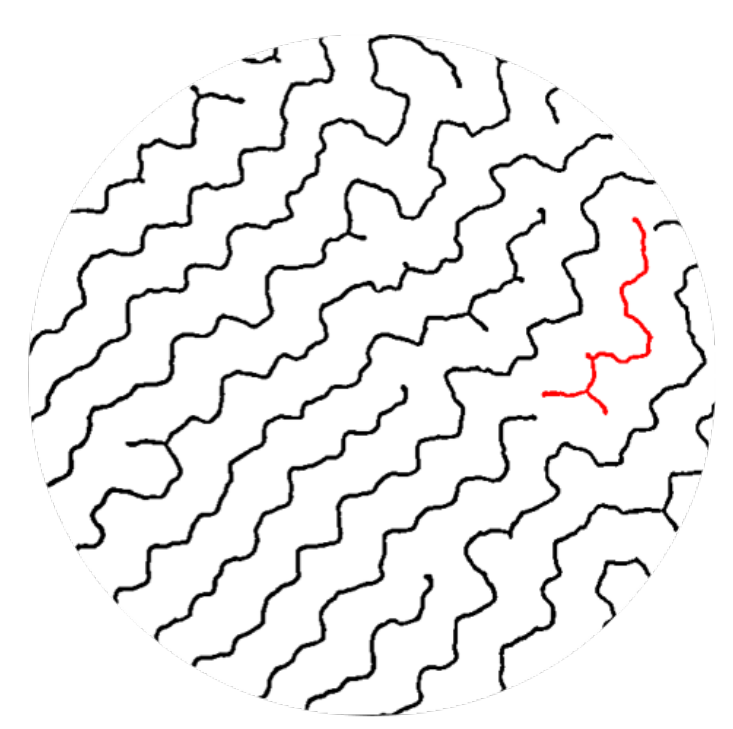

(b)

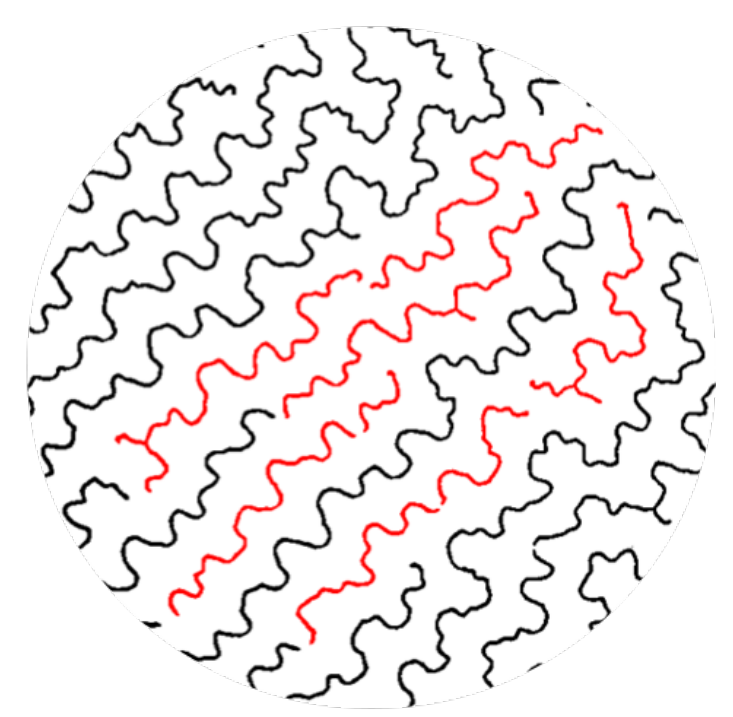

(d)

Figure 5.45: Four slices from core_1b - chamber 3 with pillars considered for analysis coloured red. (a) slice 20, (b) slice 40, (c) slice 60, (d) slice 80. 


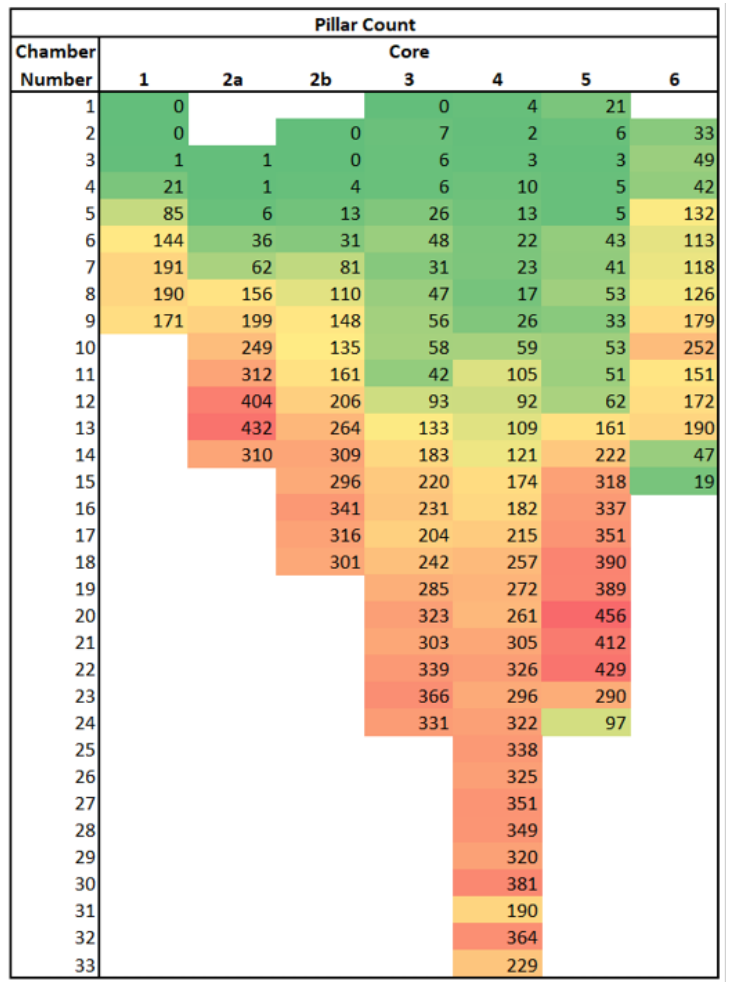

\begin{tabular}{|c|c|c|c|c|c|c|c|}
\hline \multicolumn{8}{|c|}{ Pillar Area Mean $\left(\mu \mathrm{m}^{2}\right)$} \\
\hline \multirow{2}{*}{\begin{tabular}{l|} 
Chamber \\
Number
\end{tabular}} & \multicolumn{7}{|c|}{ Core } \\
\hline & 1 & $2 \mathbf{a}$ & $2 b$ & 3 & 4 & 5 & 6 \\
\hline 1 & & & & & 19299 & 16921 & \\
\hline 2 & & & & 40913 & 6539 & 31016 & 39711 \\
\hline 3 & 10261 & 3592 & & 21051 & 9844 & 20180 & 84979 \\
\hline 4 & 96580 & 447 & 24471 & 31734 & 85323 & 15312 & 93453 \\
\hline 5 & 216980 & 20753 & 101746 & 153585 & 46134 & 28254 & 228259 \\
\hline 6 & 214269 & 121946 & 123471 & 186888 & 96583 & 95044 & 207326 \\
\hline 7 & 207859 & 150996 & 210805 & 95952 & 79017 & 127931 & 205770 \\
\hline 8 & 196268 & 233984 & 210544 & 173458 & 78205 & 185551 & 212203 \\
\hline 9 & 230562 & 224678 & 203198 & 225336 & 103313 & 132792 & 250903 \\
\hline 10 & & 216399 & 203921 & 199068 & 146565 & 175445 & 276767 \\
\hline 11 & & 255311 & 218187 & 192675 & 205032 & 175826 & 204179 \\
\hline 12 & & 248083 & 229490 & 206691 & 191823 & 178094 & 225095 \\
\hline 13 & & 282529 & 245489 & 233964 & 187816 & 241401 & 201163 \\
\hline 14 & & 258674 & 232440 & 234123 & 175956 & 212098 & 41339 \\
\hline 15 & & & 224089 & 222288 & 206737 & 229529 & 11814 \\
\hline 16 & & & 210569 & 211478 & 206724 & 249435 & \\
\hline 17 & & & 198799 & 212691 & 176452 & 260715 & \\
\hline 18 & & & 233381 & 194628 & 195981 & 236551 & \\
\hline 19 & & & & 233875 & 209839 & 211969 & \\
\hline 20 & & & & 209623 & 243854 & 261573 & \\
\hline 21 & & & & 214947 & 218996 & 262478 & \\
\hline 22 & & & & 191796 & 218841 & 298449 & \\
\hline 23 & & & & 195559 & 179633 & 205131 & \\
\hline 24 & & & & 213741 & 185282 & 43251 & \\
\hline 25 & & & & & 199102 & & \\
\hline 26 & & & & & 194153 & & \\
\hline 27 & & & & & 189686 & & \\
\hline 28 & & & & & 176690 & & \\
\hline 29 & & & & & 160009 & & \\
\hline 30 & & & & & 170042 & & \\
\hline 31 & & & & & 76689 & & \\
\hline 32 & & & & & 130698 & & \\
\hline 33 & & & & & 87058 & & \\
\hline
\end{tabular}

\begin{tabular}{|c|c|c|c|c|c|c|c|}
\hline \multicolumn{8}{|c|}{ Material to Air (\%) } \\
\hline \multirow{2}{*}{\begin{tabular}{l|} 
Chamber \\
Number
\end{tabular}} & \multicolumn{7}{|c|}{ Core } \\
\hline & 1 & $2 \mathbf{a}$ & $2 b$ & 3 & 4 & 5 & 6 \\
\hline 1 & 10.91 & & & 11.07 & 10.13 & 6.52 & \\
\hline 2 & 9.28 & & 10.84 & 10.24 & 8.72 & 8.72 & 8.01 \\
\hline 3 & 9.38 & 11.21 & 10.70 & 10.47 & 8.86 & 8.85 & 8.48 \\
\hline 4 & 8.50 & 8.72 & 9.91 & 12.31 & 9.20 & 9.51 & 10.29 \\
\hline 5 & 8.82 & 8.89 & 9.34 & 10.91 & 9.40 & 9.40 & 11.15 \\
\hline 6 & 8.85 & 8.63 & 10.05 & 10.91 & 8.99 & 9.36 & 10.81 \\
\hline 7 & 8.92 & 9.55 & 10.68 & 10.75 & 8.97 & 8.99 & 10.99 \\
\hline 8 & 8.91 & 9.12 & 10.86 & 10.64 & 9.31 & 10.97 & 11.82 \\
\hline 9 & 10.35 & 8.46 & 9.41 & 12.44 & 8.12 & 11.20 & 10.68 \\
\hline 10 & & 8.05 & 9.79 & 12.08 & 9.07 & 10.82 & 11.18 \\
\hline 11 & & 8.90 & 10.81 & 12.50 & 9.32 & 10.36 & 9.17 \\
\hline 12 & & 8.27 & 10.25 & 11.28 & 9.84 & 10.49 & 9.56 \\
\hline 13 & & 9.84 & 9.62 & 10.70 & 9.15 & 9.70 & 10.08 \\
\hline 14 & & 8.56 & 9.29 & 9.62 & 8.11 & 8.29 & 11.52 \\
\hline 15 & & & 8.93 & 9.13 & 9.04 & 8.77 & 14.49 \\
\hline 16 & & & 8.26 & 8.58 & 8.80 & 9.20 & \\
\hline 17 & & & 8.28 & 8.89 & 7.61 & 9.39 & \\
\hline 18 & & & 9.54 & 8.21 & 7.98 & 8.74 & \\
\hline 19 & & & & 8.89 & 8.49 & 7.88 & \\
\hline 20 & & & & 7.76 & 9.74 & 9.28 & \\
\hline 21 & & & & 8.17 & 8.82 & 9.83 & \\
\hline 22 & & & & 7.09 & 8.07 & 11.67 & \\
\hline 23 & & & & 7.18 & 7.07 & 12.99 & \\
\hline 24 & & & & 8.31 & 6.79 & 14.23 & \\
\hline 25 & & & & & 7.19 & & \\
\hline 26 & & & & & 7.68 & & \\
\hline 27 & & & & & 6.99 & & \\
\hline 28 & & & & & 6.73 & & \\
\hline 29 & & & & & 7.03 & & \\
\hline 30 & & & & & 7.31 & & \\
\hline 31 & & & & & 8.42 & & \\
\hline 32 & & & & & 8.63 & & \\
\hline 33 & & & & & 10.31 & & \\
\hline
\end{tabular}

\begin{tabular}{|c|c|c|c|c|c|c|c|}
\hline \multicolumn{8}{|c|}{ Perimeter $(\mu \mathrm{m})$} \\
\hline \multirow{2}{*}{$\begin{array}{l}\text { Chamber } \\
\text { Number }\end{array}$} & \multicolumn{7}{|c|}{ Core } \\
\hline & 1 & 2a & $2 b$ & 3 & 4 & 5 & 6 \\
\hline 1 & & & & & 979.07 & 195.16 & \\
\hline 2 & & & & 1066.58 & 701.22 & 1248.59 & 355.68 \\
\hline 3 & 1974.89 & 578.26 & & 703.38 & 854.85 & 1694.34 & 561.56 \\
\hline 4 & 1015.44 & 96.44 & 1109.65 & 1022.96 & 2310.18 & 789.64 & 730.34 \\
\hline 5 & 517.97 & 796.78 & 1792.80 & 1303.15 & 936.34 & 1470.04 & 543.35 \\
\hline 6 & 331.79 & 797.06 & 879.54 & 877.94 & 1254.46 & 618.67 & 591.90 \\
\hline 7 & 231.49 & 606.93 & 581.48 & 702.01 & 1006.69 & 898.78 & 545.78 \\
\hline 8 & 225.24 & 379.17 & 395.85 & 862.55 & 1187.03 & 867.05 & 471.95 \\
\hline 9 & 267.34 & 302.12 & 316.19 & 809.53 & 1283.69 & 971.59 & 434.42 \\
\hline 10 & & 225.07 & 339.73 & 689.39 & 688.00 & 818.16 & 310.74 \\
\hline 11 & & 209.52 & 293.26 & 905.94 & 512.50 & 885.55 & 407.29 \\
\hline 12 & & 164.94 & 242.13 & 482.37 & 545.27 & 725.07 & 383.27 \\
\hline 13 & & 151.82 & 206.08 & 385.65 & 481.21 & 382.88 & 303.78 \\
\hline 14 & & 201.74 & 160.27 & 281.27 & 431.20 & 274.66 & 242.54 \\
\hline 15 & & & 164.79 & 245.56 & 300.68 & 191.02 & 170.91 \\
\hline 16 & & & 135.95 & 214.35 & 288.38 & 191.32 & \\
\hline 17 & & & 134.00 & 255.75 & 232.52 & 184.40 & \\
\hline 18 & & & 152.88 & 195.43 & 194.40 & 151.16 & \\
\hline 19 & & & & 189.93 & 188.94 & 139.53 & \\
\hline 20 & & & & 149.55 & 202.59 & 136.95 & \\
\hline 21 & & & & 155.08 & 164.87 & 145.26 & \\
\hline 22 & & & & 127.16 & 156.61 & 163.83 & \\
\hline 23 & & & & 122.86 & 143.36 & 168.16 & \\
\hline 24 & & & & 142.87 & 132.81 & 101.91 & \\
\hline 25 & & & & & 134.17 & & \\
\hline 26 & & & & & 142.50 & & \\
\hline 27 & & & & & 133.99 & & \\
\hline 28 & & & & & 130.70 & & \\
\hline 29 & & & & & 134.04 & & \\
\hline 30 & & & & & 122.11 & & \\
\hline 31 & & & & & 100.40 & & \\
\hline 32 & & & & & 87.07 & & \\
\hline 33 & & & & & 96.59 & & \\
\hline
\end{tabular}

Figure 5.46: Pillar counts, mean pillar area, material to air percentages and mean pillar perimeters for each chamber of seven sample cores with conditional formatting. 


\begin{tabular}{|c|c|c|c|c|c|c|c|}
\hline \multicolumn{8}{|c|}{ Feret $(\mu \mathrm{m})$} \\
\hline \multirow{2}{*}{$\begin{array}{l}\text { Chamber } \\
\text { Number }\end{array}$} & \multicolumn{7}{|c|}{ Core } \\
\hline & 1 & $2 \mathbf{a}$ & $2 \mathrm{~b}$ & 3 & 4 & 5 & 6 \\
\hline 1 & & & & & 451.95 & 86.92 & \\
\hline 2 & & & & 363.18 & 265.75 & 518.23 & 152.06 \\
\hline 3 & 546.93 & 261.74 & & 176.24 & 256.52 & 441.06 & 224.56 \\
\hline 4 & 299.53 & 43.60 & 278.63 & 260.99 & 431.12 & 234.17 & 245.97 \\
\hline 5 & 180.31 & 223.77 & 387.21 & 281.13 & 252.48 & 381.22 & 192.20 \\
\hline 6 & 132.50 & 228.53 & 229.62 & 225.45 & 303.37 & 191.32 & 208.84 \\
\hline 7 & 96.55 & 176.58 & 181.60 & 225.74 & 274.15 & 234.98 & 199.25 \\
\hline 8 & 91.21 & 131.88 & 141.14 & 257.73 & 258.25 & 248.81 & 184.50 \\
\hline 9 & 104.16 & 112.56 & 119.25 & 223.69 & 306.37 & 274.26 & 168.42 \\
\hline 10 & & 86.83 & 129.37 & 204.20 & 219.83 & 244.96 & 119.28 \\
\hline 11 & & 78.52 & 112.18 & 277.03 & 163.61 & 279.22 & 163.67 \\
\hline 12 & & 62.68 & 92.84 & 168.36 & 175.31 & 198.53 & 145.19 \\
\hline 13 & & 58.49 & 80.61 & 137.66 & 159.08 & 127.94 & 115.69 \\
\hline 14 & & 78.29 & 65.42 & 107.07 & 143.26 & 93.77 & 85.27 \\
\hline 15 & & & 67.19 & 92.89 & 108.60 & 68.12 & 55.86 \\
\hline 16 & & & 55.39 & 83.96 & 106.28 & 69.28 & \\
\hline 17 & & & 53.30 & 96.73 & 87.60 & 64.81 & \\
\hline 18 & & & 60.55 & 75.92 & 73.09 & 54.64 & \\
\hline 19 & & & & 71.37 & 70.87 & 51.73 & \\
\hline 20 & & & & 57.72 & 74.60 & 51.48 & \\
\hline 21 & & & & 59.75 & 61.66 & 55.03 & \\
\hline 22 & & & & 48.74 & 58.78 & 60.46 & \\
\hline 23 & & & & 45.95 & 54.78 & 58.74 & \\
\hline 24 & & & & 54.14 & 52.32 & 39.48 & \\
\hline 25 & & & & & 50.40 & & \\
\hline 26 & & & & & 52.70 & & \\
\hline 27 & & & & & 48.82 & & \\
\hline 28 & & & & & 47.27 & & \\
\hline 29 & & & & & 45.96 & & \\
\hline 30 & & & & & 43.52 & & \\
\hline 31 & & & & & 37.23 & & \\
\hline 32 & & & & & 32.35 & & \\
\hline 33 & & & & & 34.79 & & \\
\hline
\end{tabular}

\begin{tabular}{|c|c|c|c|c|c|c|c|}
\hline \multicolumn{8}{|c|}{ Tortuosity } \\
\hline \multirow{2}{*}{\begin{tabular}{|l|} 
Chamber \\
Number
\end{tabular}} & \multicolumn{7}{|c|}{ Core } \\
\hline & 1 & $2 a$ & $2 b$ & 3 & 4 & 5 & 6 \\
\hline 1 & 1.19 & & 1.22 & 1.13 & 1.08 & 1.16 & \\
\hline 2 & 1.10 & & 1.33 & 1.45 & 1.31 & 1.18 & 1.17 \\
\hline 3 & 1.81 & 1.11 & & 1.60 & 1.41 & 1.44 & 1.22 \\
\hline 4 & 1.46 & 1.11 & 1.61 & 1.70 & 1.97 & 1.63 & 1.37 \\
\hline 5 & 1.34 & 1.59 & 1.93 & 1.71 & 1.57 & 1.77 & 1.35 \\
\hline 6 & 1.23 & 1.59 & 1.74 & 1.55 & 1.72 & 1.47 & 1.31 \\
\hline 7 & 1.20 & 1.47 & 1.47 & 1.38 & 1.55 & 1.56 & 1.30 \\
\hline 8 & 1.24 & 1.36 & 1.32 & 1.46 & 1.61 & 1.50 & 1.24 \\
\hline 9 & 1.29 & 1.29 & 1.26 & 1.57 & 1.74 & 1.48 & 1.26 \\
\hline 10 & & 1.26 & 1.26 & 1.48 & 1.43 & 1.47 & 1.28 \\
\hline 11 & & 1.32 & 1.27 & 1.47 & 1.43 & 1.51 & 1.23 \\
\hline 12 & & 1.31 & 1.27 & 1.36 & 1.47 & 1.56 & 1.30 \\
\hline 13 & & 1.28 & 1.23 & 1.33 & 1.43 & 1.40 & 1.32 \\
\hline 14 & & 1.26 & 1.22 & 1.27 & 1.39 & 1.40 & 1.38 \\
\hline 15 & & & 1.22 & 1.29 & 1.34 & 1.37 & 1.53 \\
\hline 16 & & & 1.23 & 1.25 & 1.31 & 1.36 & \\
\hline 17 & & & 1.26 & 1.28 & 1.29 & 1.40 & \\
\hline 18 & & & 1.25 & 1.27 & 1.30 & 1.37 & \\
\hline 19 & & & & 1.32 & 1.31 & 1.33 & \\
\hline 20 & & & & 1.30 & 1.32 & 1.32 & \\
\hline 21 & & & & 1.29 & 1.32 & 1.31 & \\
\hline 22 & & & & 1.30 & 1.32 & 1.32 & \\
\hline 23 & & & & 1.33 & 1.29 & 1.36 & \\
\hline 24 & & & & 1.31 & 1.26 & 1.26 & \\
\hline 25 & & & & & 1.32 & & \\
\hline 26 & & & & & 1.33 & & \\
\hline 27 & & & & & 1.34 & & \\
\hline 28 & & & & & 1.35 & & \\
\hline 29 & & & & & 1.42 & & \\
\hline 30 & & & & & 1.37 & & \\
\hline 31 & & & & & 1.34 & & \\
\hline 32 & & & & & 1.31 & & \\
\hline 33 & & & & & 1.35 & & \\
\hline
\end{tabular}

\begin{tabular}{|c|c|c|c|c|c|c|c|}
\hline \multicolumn{8}{|c|}{ Pillar Thickness Mean $(\mu \mathrm{m})$} \\
\hline \multirow{2}{*}{$\begin{array}{l}\text { Chamber } \\
\text { Number }\end{array}$} & \multicolumn{7}{|c|}{ Core } \\
\hline & 1 & $2 a$ & $2 \mathbf{b}$ & 3 & 4 & 5 & 6 \\
\hline 1 & 15.08 & & & 13.50 & 11.79 & 10.19 & \\
\hline 2 & 12.45 & & 13.47 & 13.95 & 10.63 & 9.71 & 8.16 \\
\hline 3 & 12.68 & 16.12 & 14.64 & 12.90 & 9.90 & 9.28 & 7.93 \\
\hline 4 & 11.66 & 12.64 & 13.16 & 13.42 & 8.79 & 9.33 & 7.17 \\
\hline 5 & 12.20 & 12.26 & 10.83 & 11.97 & 8.70 & 7.75 & 7.90 \\
\hline 6 & 11.65 & 11.37 & 11.94 & 11.80 & 9.15 & 9.08 & 7.59 \\
\hline 7 & 11.97 & 10.76 & 11.95 & 11.54 & 8.98 & 9.02 & 7.88 \\
\hline 8 & 11.74 & 10.37 & 12.86 & 10.78 & 8.52 & 10.34 & 8.58 \\
\hline 9 & 12.60 & 9.88 & 11.88 & 11.72 & 8.11 & 10.64 & 7.01 \\
\hline 10 & & 10.17 & 12.16 & 13.26 & 8.91 & 10.37 & 6.77 \\
\hline 11 & & 10.52 & 12.51 & 13.08 & 9.41 & 10.20 & 7.87 \\
\hline 12 & & 9.92 & 12.19 & 12.23 & 8.88 & 9.43 & 8.01 \\
\hline 13 & & 12.04 & 11.85 & 12.33 & 8.85 & 8.71 & 8.26 \\
\hline 14 & & 11.20 & 12.56 & 12.39 & 8.73 & 9.21 & 8.64 \\
\hline 15 & & & 12.74 & 10.33 & 9.39 & 10.06 & 8.76 \\
\hline 16 & & & 12.60 & 11.68 & 9.18 & 9.83 & \\
\hline 17| & & & 13.04 & 11.09 & 9.21 & 9.86 & \\
\hline 18 & & & 13.31 & 11.31 & 10.06 & 10.26 & \\
\hline 19 & & & & 10.74 & 10.36 & 10.26 & \\
\hline 20 & & & & 11.20 & 11.51 & 10.92 & \\
\hline 21 & & & & 12.05 & 11.43 & 11.64 & \\
\hline 22 & & & & 12.26 & 11.13 & 11.43 & \\
\hline 23 & & & & 11.78 & 11.51 & 11.64 & \\
\hline 24 & & & & 11.99 & 11.83 & 12.36 & \\
\hline 25 & & & & & 12.00 & & \\
\hline 26 & & & & & 11.35 & & \\
\hline 27| & & & & & 10.79 & & \\
\hline 28 & & & & & 10.39 & & \\
\hline 29 & & & & & 10.07 & & \\
\hline 30 & & & & & 10.02 & & \\
\hline 31 & & & & & 11.22 & & \\
\hline 32 & & & & & 12.35 & & \\
\hline 33 & & & & & 10.99 & & \\
\hline
\end{tabular}

\begin{tabular}{|c|c|c|c|c|c|c|c|}
\hline \multicolumn{8}{|c|}{ Spacing Mean $(\mu \mathrm{m})$} \\
\hline \multirow{2}{*}{$\begin{array}{l}\text { Chamber } \\
\text { Number }\end{array}$} & \multicolumn{7}{|c|}{ Core } \\
\hline & 1 & $2 a$ & $2 \mathrm{~b}$ & 3 & 4 & 5 & 6 \\
\hline 1 & 113.89 & & & 93.60 & 98.06 & 94.88 & \\
\hline 2 & 129.65 & & 141.16 & 115.21 & 111.77 & 102.70 & 56.94 \\
\hline 3 & 122.82 & 119.25 & 122.59 & 102.54 & 99.11 & 83.60 & 65.85 \\
\hline 4 & 114.09 & 112.05 & 105.14 & 86.84 & 63.40 & 79.71 & 29.32 \\
\hline 5 & 104.79 & 110.24 & 91.67 & 87.29 & 69.29 & 41.06 & 37.50 \\
\hline 6 & 94.05 & 97.61 & 89.54 & 82.92 & 83.43 & 80.23 & 36.24 \\
\hline 7 & 85.52 & 81.32 & 83.15 & 85.64 & 82.48 & 82.43 & 37.09 \\
\hline 8 & 85.83 & 69.93 & 79.95 & 82.08 & 70.28 & 65.47 & 39.07 \\
\hline 9 & 80.14 & 73.35 & 84.41 & 76.94 & 81.95 & 72.42 & 23.19 \\
\hline 10 & & 71.41 & 80.79 & 82.34 & 81.52 & 70.95 & 18.78 \\
\hline 11 & & 68.48 & 74.85 & 81.68 & 78.99 & 72.05 & 46.96 \\
\hline 12 & & 63.38 & 68.60 & 77.23 & 56.91 & 61.87 & 50.04 \\
\hline 13 & & 65.44 & 67.65 & 78.37 & 75.77 & 50.78 & 51.35 \\
\hline 14 & & 74.11 & 69.94 & 80.39 & 80.31 & 73.36 & 49.61 \\
\hline 15 & & & 79.42 & 76.61 & 69.97 & 70.31 & 45.80 \\
\hline 16 & & & 80.28 & 81.27 & 63.94 & 64.59 & \\
\hline 17 & & & 82.21 & 79.94 & 80.16 & 66.65 & \\
\hline 18 & & & 80.00 & 82.06 & 82.62 & 72.26 & \\
\hline 19 & & & & 73.75 & 68.38 & 76.63 & \\
\hline 20 & & & & 77.95 & 64.34 & 70.49 & \\
\hline 21 & & & & 81.24 & 76.04 & 71.62 & \\
\hline 22 & & & & 87.39 & 76.56 & 64.13 & \\
\hline 23 & & & & 85.37 & 87.59 & 61.63 & \\
\hline 24 & & & & 82.22 & 91.44 & 53.52 & \\
\hline 25 & & & & & 88.46 & & \\
\hline 26 & & & & & 84.03 & & \\
\hline 27 & & & & & 87.60 & & \\
\hline 28 & & & & & 85.02 & & \\
\hline 29 & & & & & 85.33 & & \\
\hline 30 & & & & & 80.77 & & \\
\hline 31 & & & & & 78.11 & & \\
\hline 32 & & & & & 80.17 & & \\
\hline 33 & & & & & 61.39 & & \\
\hline
\end{tabular}

Figure 5.47: Mean pillar Feret lengths, mean pillar tortuosity, mean pillar thickness and mean pillar spacing for each chamber of seven sample cores with conditional formatting. 
in proximity to chambers with medium and high pillar counts, and conversely, chambers with low pillar counts are not near areas that regularly experience fluid flow.

(a)

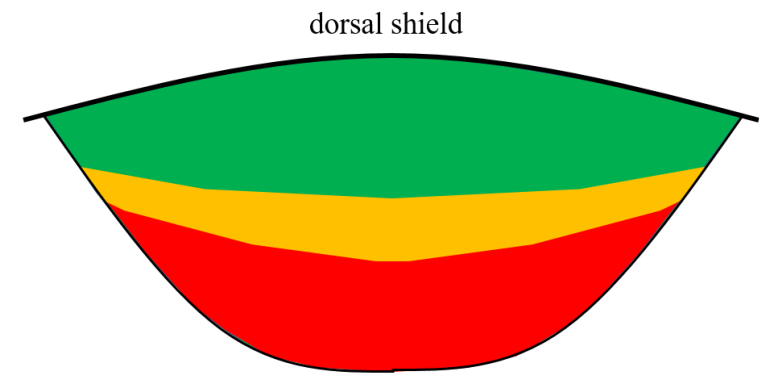

pillars per $3.14 \mathrm{~mm}^{2}$

$301-500$

$101-300$ $0-100$

(b)

dorsal shield

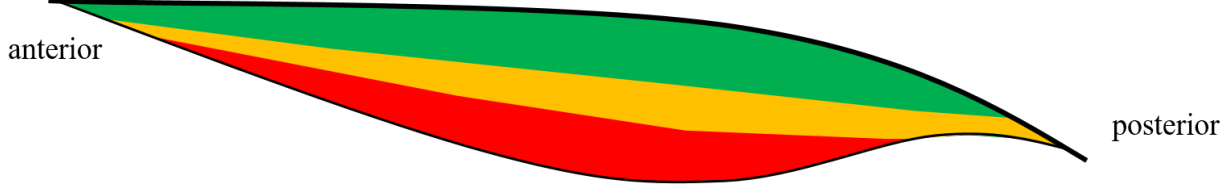

Figure 5.48: Schematic of cuttlebone indicating regions of pillar counts: (a) cross-sectional view of the cuttlebone in the transverse direction; (b) cross-sectional view in the longitudinal direction.

Figure 5.49 indicates the percentage of material-to-air generally found across the cuttlebone. The material-to-air percentage was found to be between $4 \%$ and $20 \%$ for the middle slices of each chamber in the sampled areas. There were a number of trends worth including in the framework;

- The highest percentages were found in a thin band of chambers in direct proximity to the siphonal zone. The 'contacting ridges' [224] along the siphonal edge probably contribute significantly to this observed feature.

- The lowest percentage of material within chambers is found in those close to the last mineralised septum, running along the ventral edge in the anterior direction.

- Chambers close to the centre of the cuttlebone have a slightly higher percentage of material, around $12 \%$ compared to surrounding chambers.

Figure 5.50 indicates regions of tortuosity. Chambers in proximity to the dorsal shield show very low tortuosity, i.e. close to one, and the area surrounding the last mineralised chamber also shows low pillar tortuosity. However, ventral chambers quickly become much more tortuous. Regions with highest pillar tortuosity are not near the siphonal zone.

Morphological features discussion Pillar counts are low in the top half of the cuttlebone because pillars in this region are much longer than those in more ventral regions, with varying tortuosity depending on their position relative to the dorsal shield. The length of 
(a)

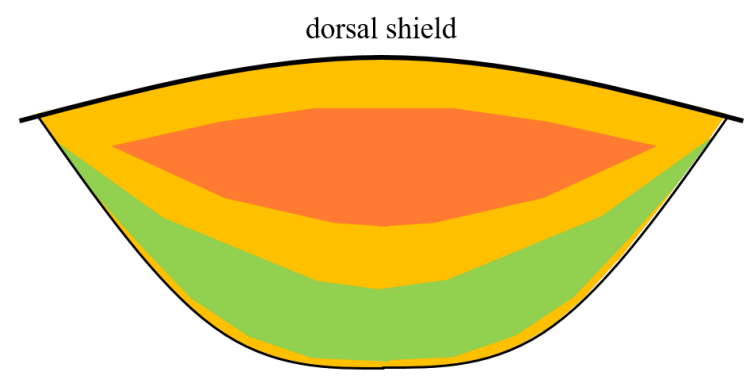

$0.14-0.20$

$0.11-0.14$

$0.09-0.11$

$0.07-0.09$

$0.04-0.07$

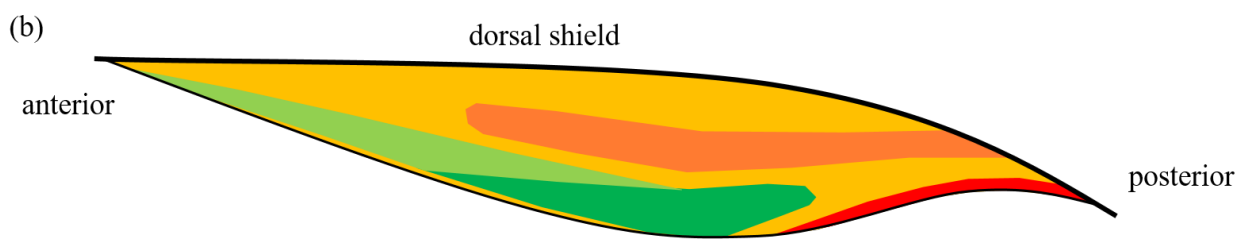

Figure 5.49: Schematic of cuttlebone indicating regions of material-to-air percentage: (a) cross-sectional view of the cuttlebone in the transverse direction; (b) cross-sectional view in the longitudinal direction.

(a)
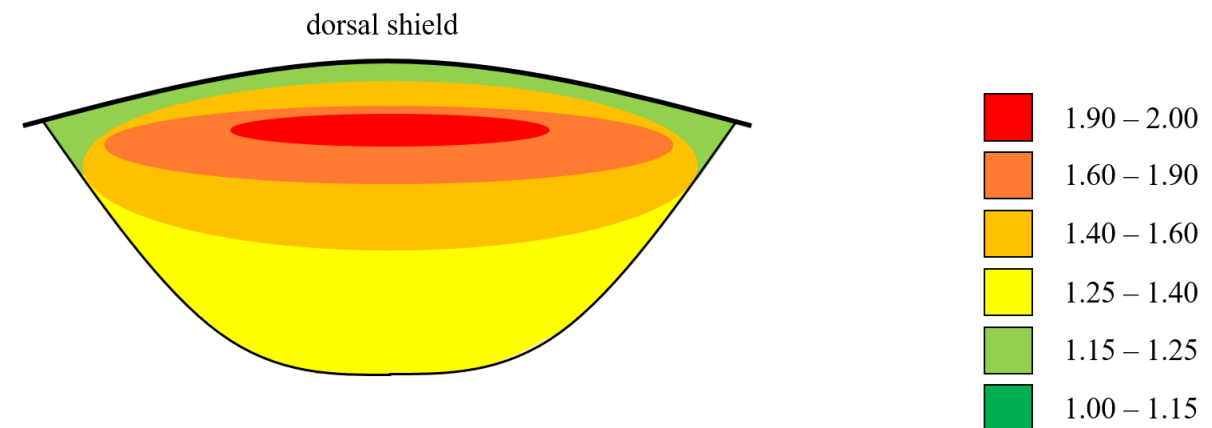

(b)

dorsal shield

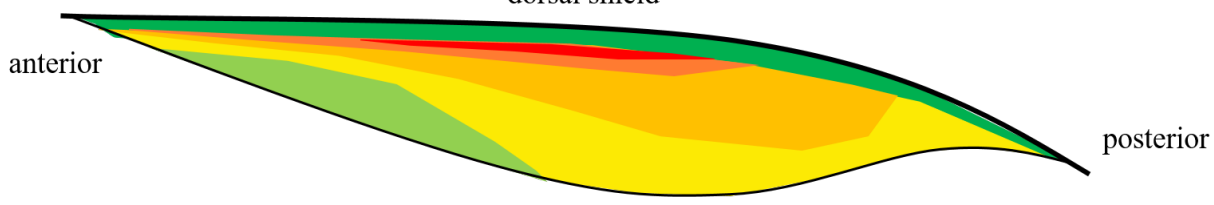

Figure 5.50: Schematic of cuttlebone indicating regions of pillar tortuosity: (a) crosssectional view of the cuttlebone in the transverse direction; (b) cross-sectional view in the longitudinal direction. 
these pillars is often not captured in the quantitative analysis as they transcend the sampling area, however their morphology is clear when images such as those in Figure 5.56 are reviewed qualitatively. Chambers in lower areas of the cuttlebone have far greater pillar counts. These numerous pillars are typically much smaller, and naturally have accompanying lower Feret lengths and perimeters. The highest pillar counts are found in core_5c, followed by core_2a. Since core_2a is located on the lateral edge of the cuttlebone, this would suggest that these edges of the cuttlebone have high pillar counts.

Denton and Gilpin [225] showed the distribution of liquid in the chambers of a cuttlebone. They showed that older chambers at the posterior would fill with liquid when submerged at various pressures, however the new chambers, and dorsal regions remain largely dry (see Figure 5.51). This distribution correlates with the pillar count schematic in Figure 5.48, suggesting a link between the number of pillars and the ability for liquid to flow through the chambers. Figure 5.52 shows slice 008 in chamber 22 of core_5c, in proximity to the siphonal zone. The pillars are numerous, tortuous and small, but also well aligned, unlike the random pillar patterns seen in other regions of the cuttlebone. This alignment would presumably allow liquid to flow almost freely in this area of the cuttlebone. This alignment and the high material-to-air percentage seen in Figure 5.49 (supported by low pillar spacing in Figure 5.47) could also suggest that the cuttlefish uses some amount of capillary action to aid with the flow of fluid in these chambers.

Cores $1 \mathrm{~b}, 2 \mathrm{~b}$ and $3 \mathrm{c}$ all show that, across all chambers, the number of pillars in each chamber remains largely unchanged from roof to floor. Cores $4 \mathrm{c}, 5 \mathrm{c}$ and $6 \mathrm{~b}$ however show a general increase in pillar counts towards the floor of almost all chambers. These cores are located near the posterior of the cuttlebone and are all in the siphonal zone. Figure 5.53 taken from a ventral chamber in core_6b identifies some splitting at the base of pillars. This accounts for the changes noted.

The majority of chambers show an initial decrease before a curved increase in the shape of material-to-air, with increased percentages of material in the beginning and ending slices in each chamber. This behaviour can be linked and explained by the 'tree shape' seen at the individual pillar level, with examples given in Figure 5.54. The pillars branch at the ceiling and show a broadening root-like structure at the floor. This is probably strength related and also linked to the way a new chamber is formed. The only chambers not generally following this trend are found in core_6b at the posterior. Pillars in these chambers also show a similar 'tree shape', however the pillars appear to split considerably towards the floor (Figure 5.25 shows how pillar counts in each chamber start increasing from midway, much sooner than in other core samples), meaning that the overall material-to-air percentage doesn't increase as sharply as seen in other cores.

The tortuosity framework in Figure 5.50 shows that the region with highest levels of tortuosity are found in the upper centre of the cuttlebone. The liquid distributions seen in [225] show that this region of the cuttlebone doesn't typically contain liquid. The tortuosity of the pillars in this and similar regions could be helping to prevent liquid penetrating 

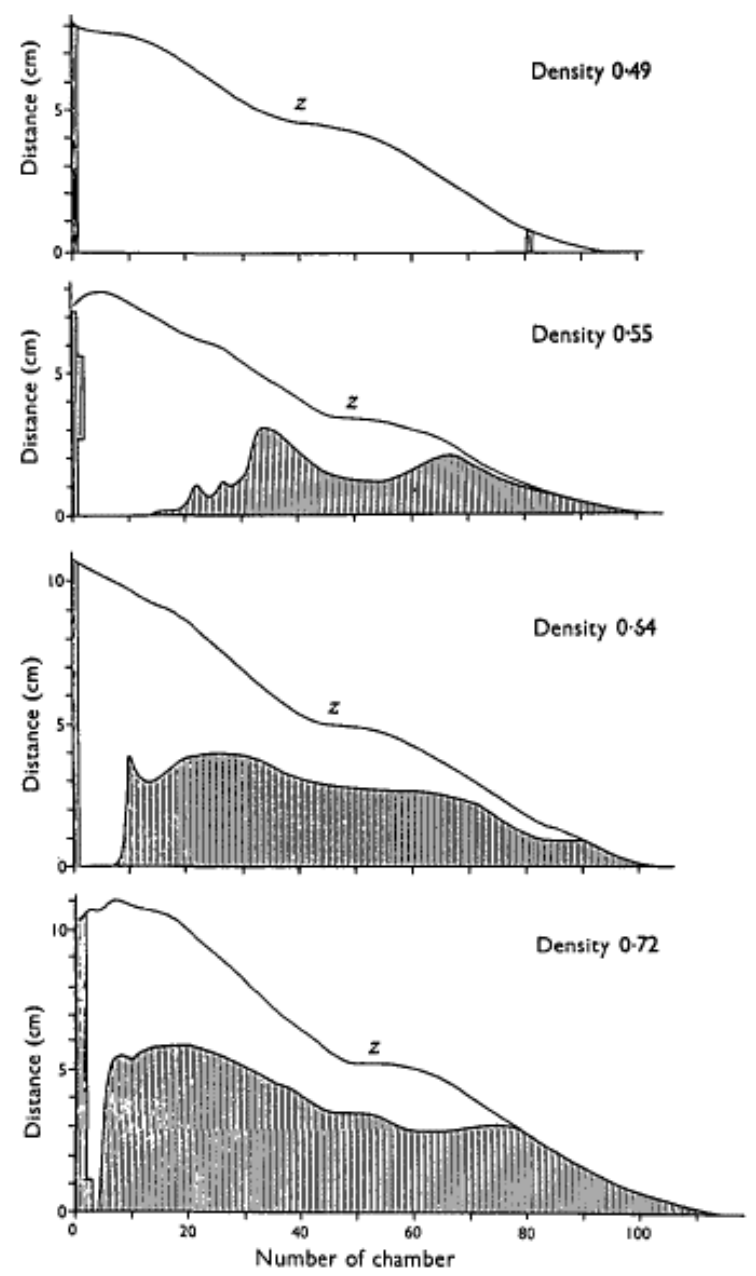

Figure 5.51: Plots showing the distribution of fluid in chambers of four cuttlebone of varying densities. Shaded areas indicate regions containing liquid. Vertical axis describes the distance along a chamber from the siphuncular surface [225]. 


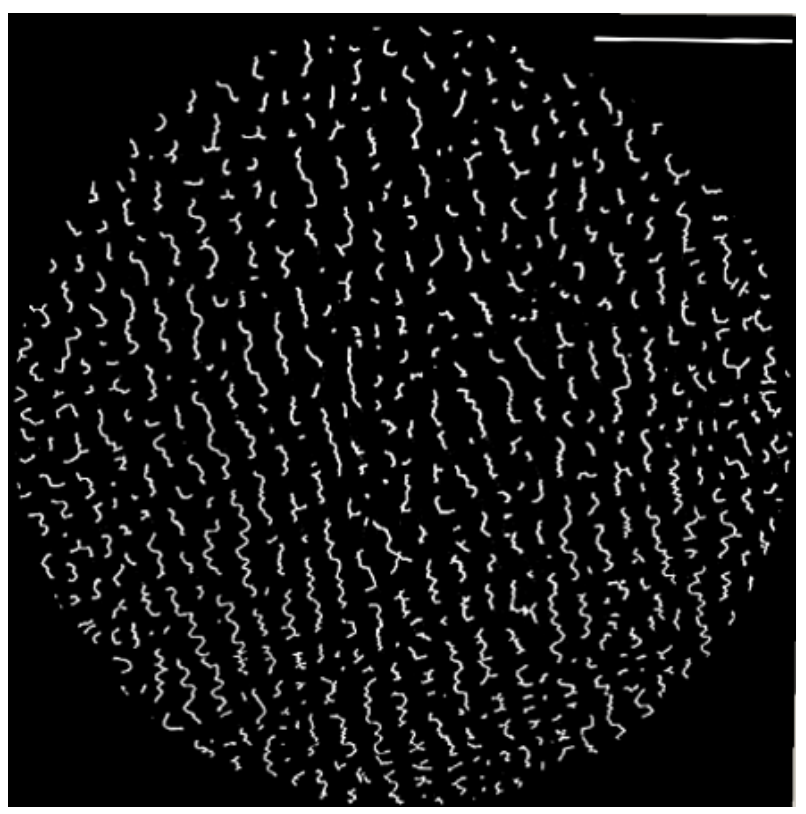

Figure 5.52: Example from core_5c chamber 22 slice 008 of the alignment of pillars in proximity to the siphonal zone.

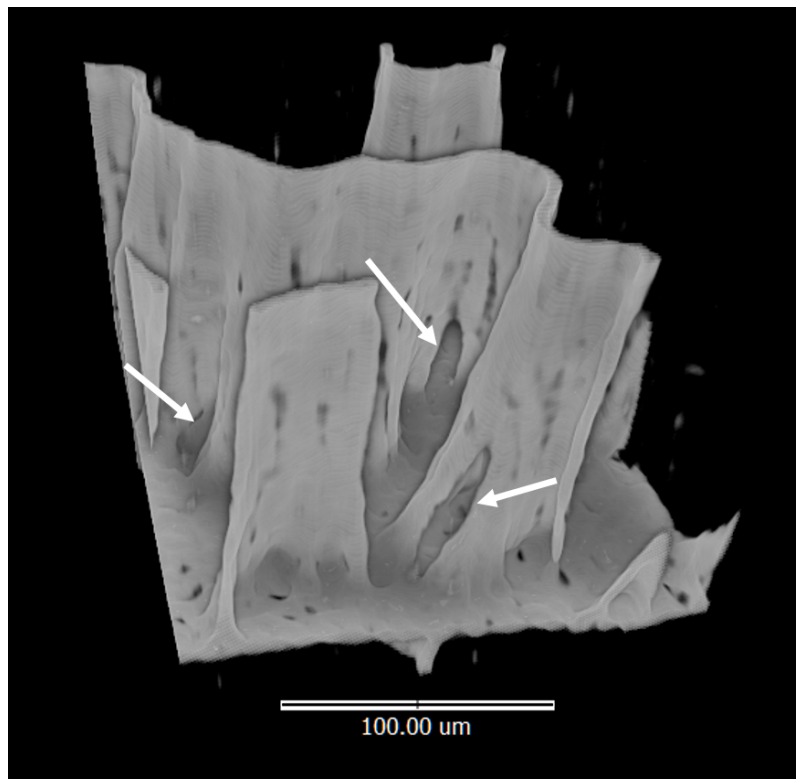

Figure 5.53: Example from core_6b chamber 10 of pillars splitting near the floor of a chamber. 


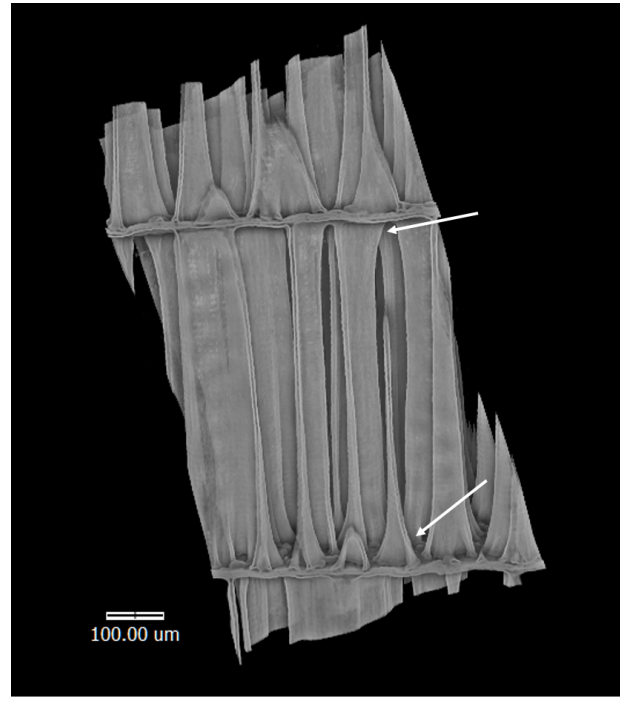

(a)

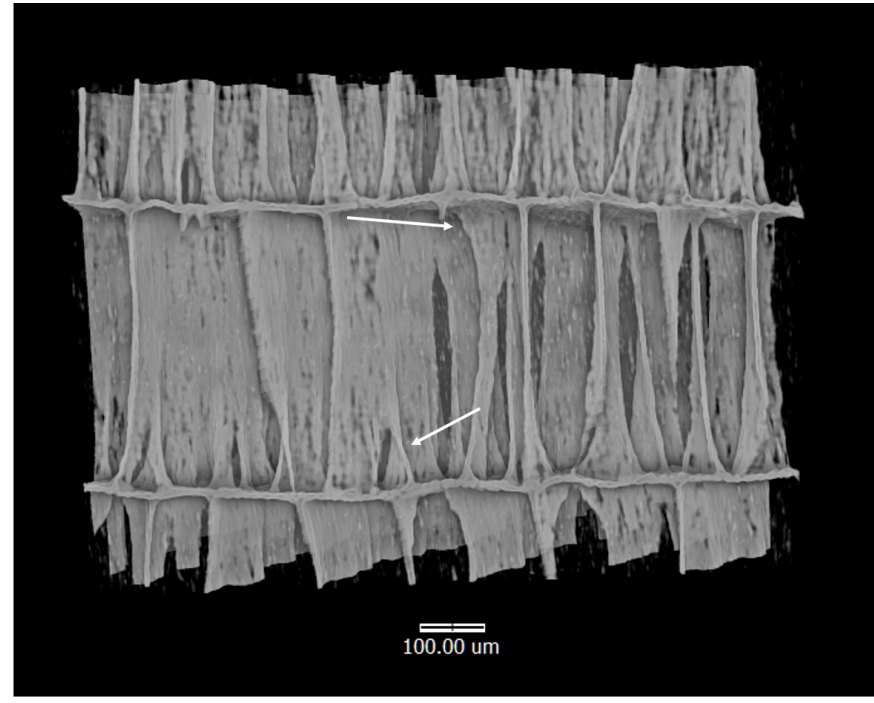

(b)

Figure 5.54: Examples of tree shaped pillars from (a) core_3c and (b) core_6b. Examples of branching and rooting are highlighted with arrows. Due to the virtual cropping of these images, partial segments of other pillars can also be seen.

these areas. Excessive liquid in these upper and anterior regions may cause the cuttlefish to become unbalanced during motion. Figure 5.55 shows two different chambers; (a) is found in core_6b in proximity to the ventral edge, (b) is found in core_3c, in the region with highest tortuosity. It is clear that liquid could flow through chamber (a) rapidly, however liquid in (b) would be slow to flow and in some cases be blocked by branching pillars. This progressive effect on chamber flooding could help the cuttlefish to regulate its buoyancy more effectively. Additionally, it may be assumed that the pillar structure in Figure 5.55(b) is stronger in bending (compressive load due to hydrostatic forces) than the structure in (a) due to an increased second moment of area, and a varying tortuosity might help with any tensile forces experienced in the structure due to the expansion of gas whilst liquid remains. This might be more relevant in deep water species of cuttlefish which experience higher pressures [191].

There is consistency across the cuttlebone with regards to the change in tortuosity within a pillar. Pillars become increasingly tortuous towards the floor of the chamber, substantially so in all but core_6b, which shows a more linear increase. This change in shape has been suggested by Checa et al. [184] as being caused by viscous fingering instability. They have reproduced experimentally the pillar shapes at the roof of a chamber, however have not been able to recreate the patterns seen at the floor, potentially due to the wettability of the surfaces being different at the two extremes, due to two different microstructures indicated in Figure 5.60(b-e).

$\mathrm{X}$-ray $\mu \mathrm{CT}$ allows for both quantitative and qualitative analysis of natural specimens. Qualitative analysis provides context and detail of specific areas, however quantitative allows 


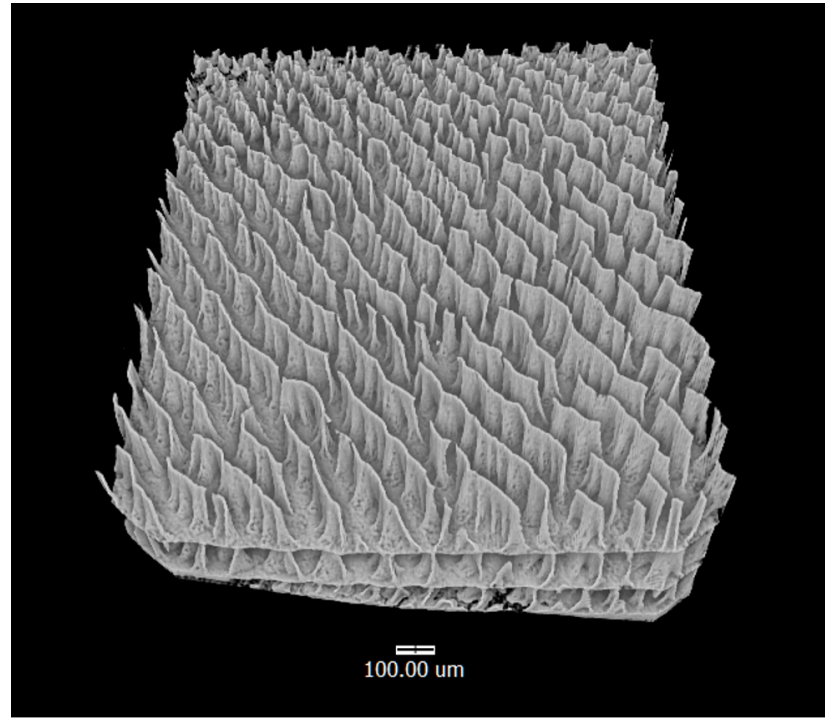

(a)

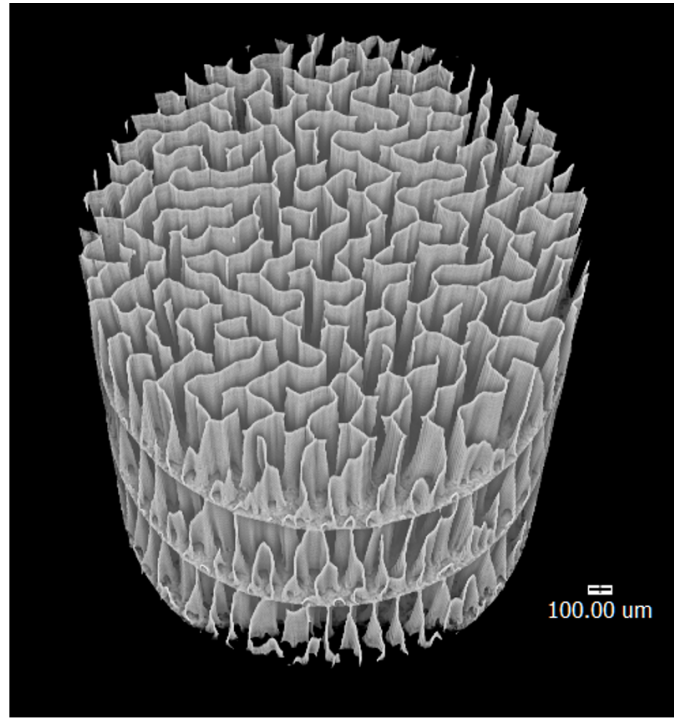

(b)

Figure 5.55: Examples of pillar alignment; (a) core_6b, (b) core_3c.

a complete picture to be built. The quantitative analysis given so far provides a good foundation of a comprehensive description of the cuttlebone. There are limitations however in the methods used during this study, such as sampling area and exclusion of incomplete data. One potential way to combat these challenges could be to incorporate a form of censoring into the results, where all pillars in the sampling area are included, but those that are incomplete are flagged. This method would also bring its own challenges however, such as pillars being considered multiple times in a sampling region. Quantitative limitations from the methods used in this study only seem to have an impact on a relatively small proportion of chambers. Therefore, by combining this approach with a qualitative description, the morphological variations across the cuttlebone are comprehensively described.

The pillar behaviour next to the dorsal shield was hard to quantify in the quantitative samples, due to the pillars transcending the regions of interest. Figure 5.56(a) gives an example of a cross-section of a cuttlebone chamber taken at the interface of the dorsal shield and lamellar matrix, from the lateral edge. This image is taken from the sample CB_w/shield (see Table 5.1). No quantitative analysis was performed on this sample. It is evident here that there is a consistent straightening of the pillars from the intersection of the dorsal shield towards the longitudinal axis of the cuttlebone. The distance, in this sample at least, of the straightened pillars is consistent across chambers. The pillars also bifurcate, maintaining consistent spacing and pillar thickness. Figure 5.50 shows that, based on limited quantifiable data, a thin band of straight pillars run along the length of the dorsal shield. Figure 5.56(b) is a multi-chamber (top two chambers) image of pillars with very low tortuosity and strong alignment anteroposteriorly. This straightening of pillars in the most dorsal chambers may be the most efficient use of material as the dorsal shield will provide 
enough compressive resistance for the surrounding region. Pillars not in the immediate vicinity need to provide more compressive strength so become more tortuous. Pillars found in older chambers at the posterior are generally straighter. When the cuttlefish is young, they inhabit in shallow depths and so pressures are lower [226]. More tortuous regions of cuttlebone potentially develop when the cuttlefish grows and is able to inhabit deeper depths.

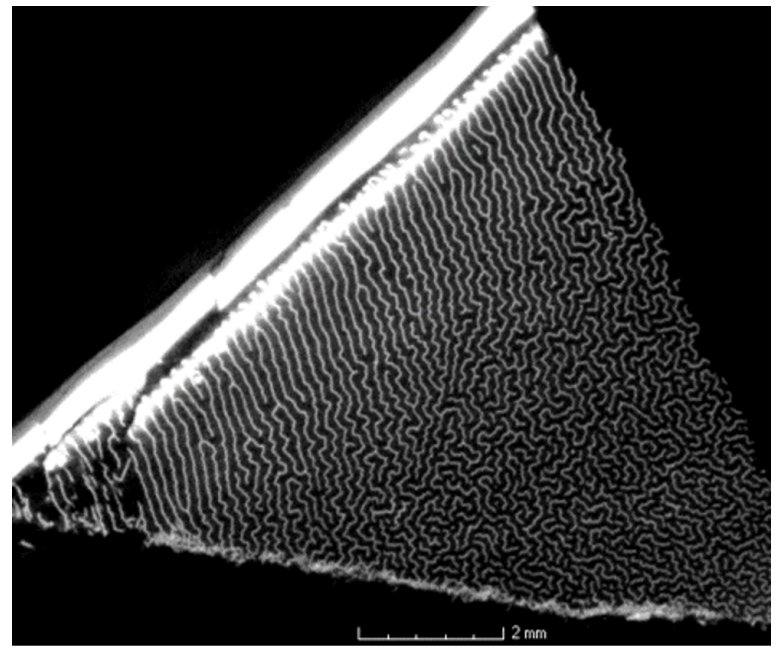

(a)

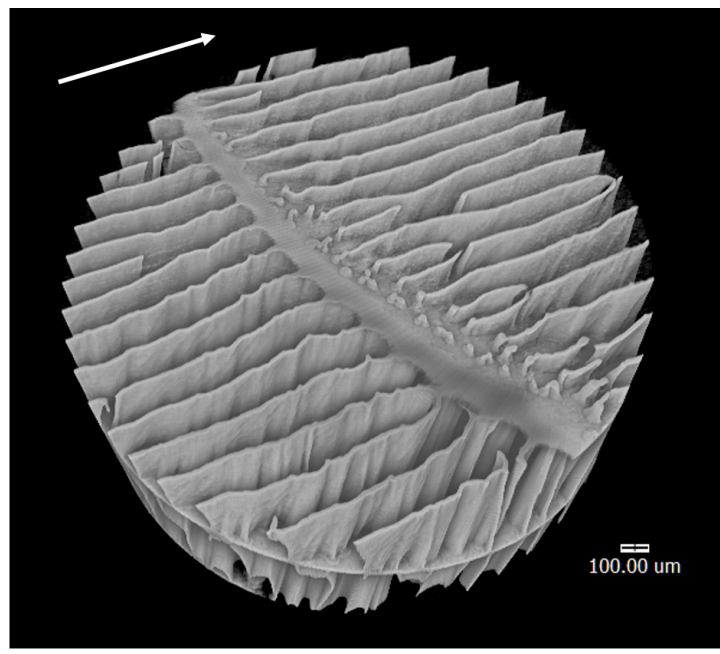

(b)

Figure 5.56: Two images of straight pillars: (a) sample $\mathrm{CB}_{-} \mathrm{w} /$ shield of the intersection of long pillars with the dorsal shield; (b) a multi-chamber image of the top chambers in core_3c. The curved line through the middle of the image is the septum. Arrow indicates anteroposterior direction.

Initial observations of the pillar formation in cuttlebone show that it bears a striking resemblance to a Turing pattern, also known as a reaction-diffusion system [227]. In particular, Figure 5.56(a) shows pillar patterns comparable to those of the angel fish stripes shown by Kondo and Asai [228]. For instance, straight pillars bifurcate whilst spacing is maintained. Close ups of these patterns are given in Figure 5.57(a), along with comparable patterns from cuttlebone (b). It is also interesting to note that these patterns can also be observed on the skin of some cuttlefish (c). Although these patterns are similar, chemical compounds acting as a morphogen would need to be identified for cuttlebone pillars to be considered a Turing pattern.

\subsubsection{Microstructural analysis}

The microstructure of cuttlebone has been studied previously [177, 183, 184, 189] (amongst others). For this work microstructural analysis is used to complement the $\mu \mathrm{CT}$ analysis by providing higher resolution of this hierarchical structure. Regions identified using $\mu \mathrm{CT}$ were investigated using both SE and BSE imaging. 


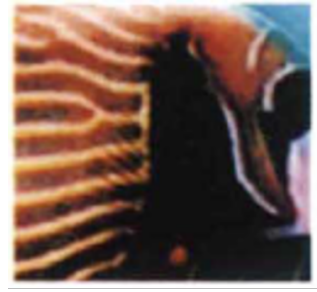

(a.1)

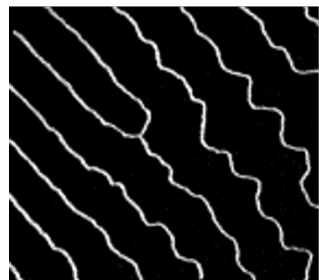

(b.1)

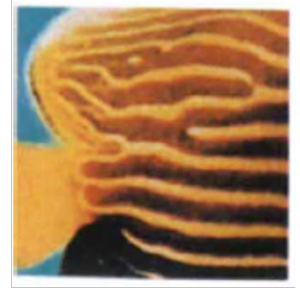

(a.2)

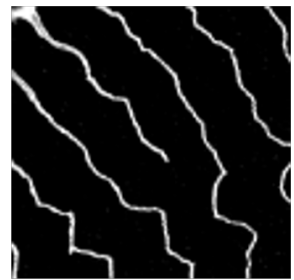

(b.2)

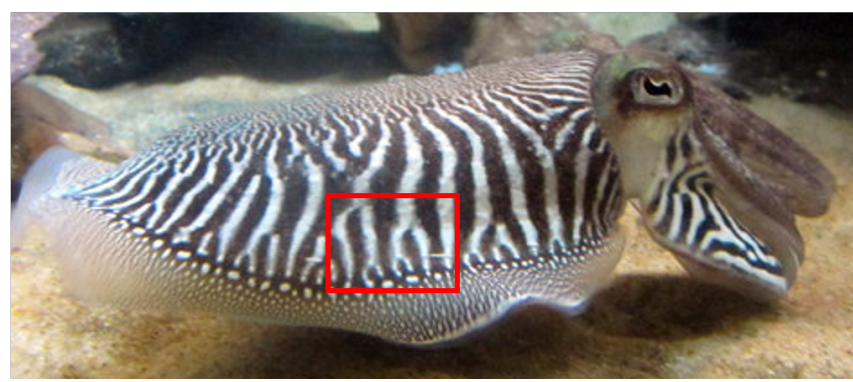

(c)

Figure 5.57: Straight line and bifurcation patterns seen in nature: (a) images of the stripe pattern of Pomacanthus imperator [228], (b) cuttlebone pillars in proximity to the dorsal shield, (c) cuttlefish skin with box highlighting comparable patterns to (a) and (b).

The microstructure of dorsal shield and intersection with lamellar matrix is shown in Figure 5.58. Image (a) shows the focus region. There are three distinct layers of microstructure in the dorsal shield, seen in (b) and (c). The outermost and innermost layers are formed of prismatic tubercles, whereas the middle layer has a lamellar structure. This middle layer varies in height depending on its position to the longitudinal axis [229]. The gap seen in (b) and (c) between the pillars and dorsal shield is also seen in Figure 5.56(a). Image (d) shows a polished section of the most central part of the dorsal shield using BSE imaging which identifies a darker band indicating clear elemental differences. This is interesting and chemical analysis will be performed on this section.

SEM images of pillars are shown in Figure 5.59. The tree shaped pillars identified in the quantitative analysis are clearly shown in image (a) with less branching at the top of the pillars. This image highlights the limitations of capturing the pillar morphology that has been shown using 3D analysis, such as pillar count, length and tortuosity. Image (b) demonstrates the horizontal organic membranes which are remnants of the mineralisation process [184].

The microstructure of septa is shown in Figure 5.60. Images (a) and (b) show the pillars joining septa. The lower pillars show a continuous transition with the chamber roof, however the upper pillars' connection with the septa is far more disjointed. The upper pillars also show knuckle-like globules at their base, which could be a factor in the increasing material-to-air percentage at the floor of chambers seen across the core samples in Figures 5.28-5.34. Horizontal lines seen on the pillars in Figure 5.60(a) are due to growth increments [177]. Images (b-d) show increasingly higher magnifications of septa. The chamber floor and roof have a lamello-fibrillar and prismatic microstructure respectively. The prismatic 


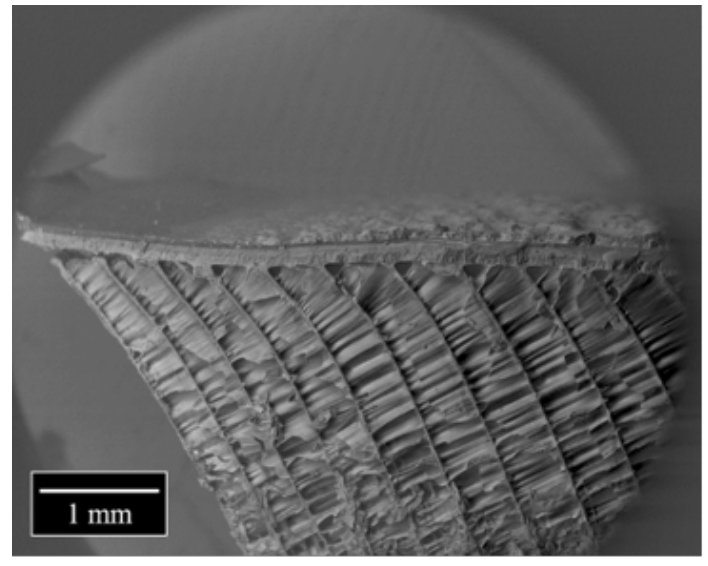

(a)

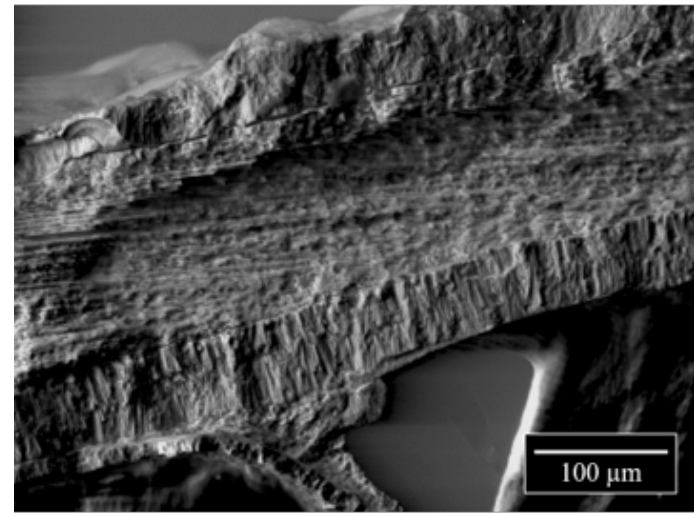

(c)

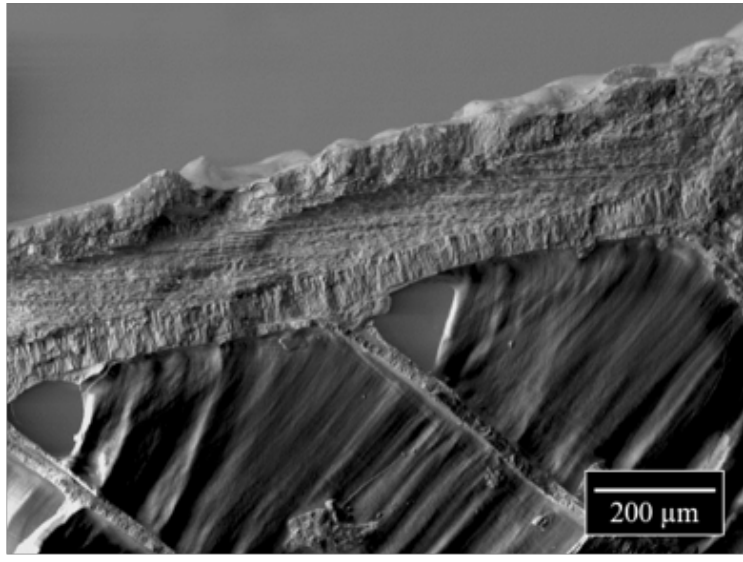

(b)

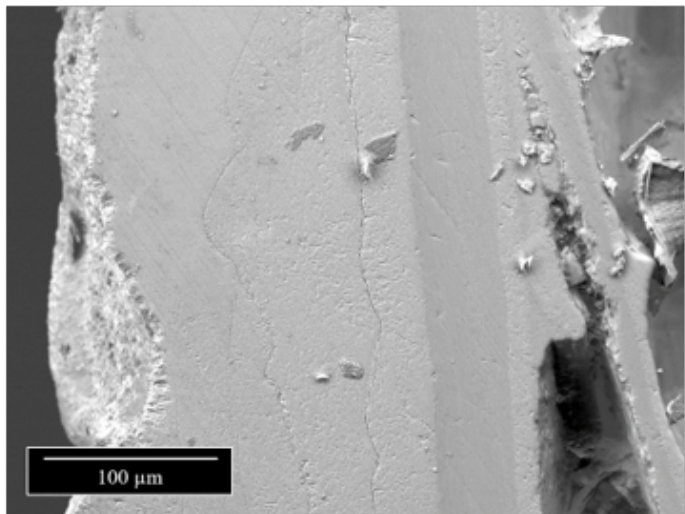

(d)

Figure 5.58: Secondary and backscattered electron SEM images of the dorsal shield and intersection with the lamellar matrix: (a) at lateral edge; (b) higher magnification of two chambers intersecting the shield; (c) three distinct bands of differing microstructures; (d) BSE image of polished section highlighting a change in atomic density.

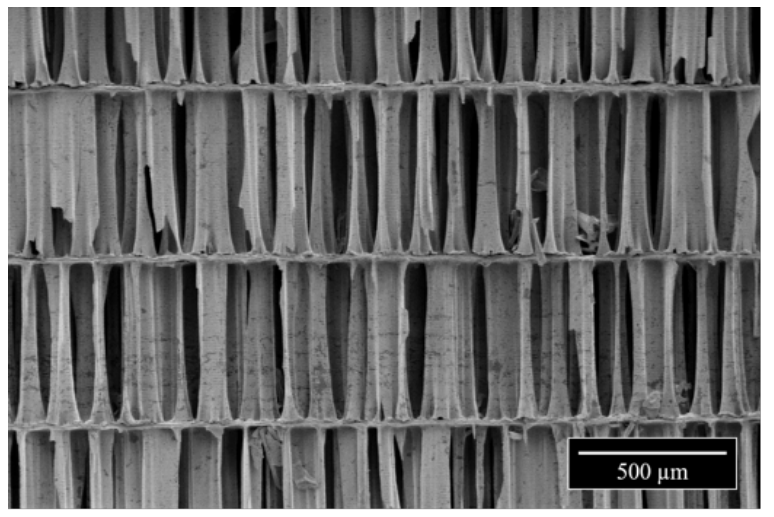

(a)

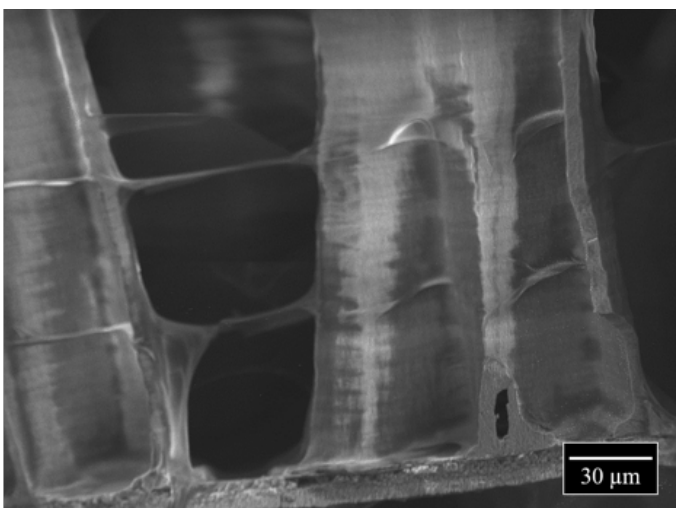

(b)

Figure 5.59: SEM images of pillars: (a) transverse view of the lamellar matrix; (b) pillars with the interconnected organic matrix. Dorsal shield to the top of all images. 
structure of the chamber roof is continuous into the pillars, with the aragonite fibres running perpendicular to the septum and parallel to the pillar height. The chamber floor conversely is made up of bundles of aragonite fibres which appear to change orientation at a high angle in different planes. A simple schematic representing this behaviour is shown in (f). This stacking of aragonite fibres in different orientations is an example of a helical structural design element described in section 2.1.2. Helical designs are linked to increasing toughness due to increased resistance to crack propagation. A reason this structural design element is found in this part of the cuttlebone could be to help prevent the septum experiencing structural failure.

Figure 5.61 shows polished sections of septa using a FIB, both in the transverse (a) and dorsoventral (b) directions. The chamber floor, at bottom of (a), shows a porous structure in comparison to the chamber roof and the section of a pillar. At a $90^{\circ}$ rotation to this, i.e. in the dorsal plane, through the chamber floor, fibres can be distinguished and the pores have a tube-like structure. This is in agreement with the observations made from Figure 5.60. The crystallographic orientation of these bundles of aragonite fibres in the septa is investigated in the next section using EBSD.

\subsubsection{EBSD}

The crystallographic orientation of aragonite in the dorsal shield and septa were investigated using EBSD. The settings used to acquire EBSD maps are given in Table 5.5. Both maps were aligned with the septum parallel to the X-direction.

Band contrast maps for dorsal shield (a) and septum (b) maps are displayed in Figure 5.62. The dorsal shield initial index success rate was $64 \%$, similarly the septum index rate was also $64 \%$. In both images, brighter areas indicate those that diffract and index well. Due to the difficulty in obtaining consistently indexable patterns, only two maps were acquired the outer portion of the dorsal shield (shown in Figure 5.58) and the full height of a septum.

EBSD maps were subject to a minimal clean-up algorithm procedure, removing any wild spikes and growing grains to the nearest 6 neighbours. Only minimal cleaning was applied to maintain a representative map, as some regions contain no indexed points.

Figure 5.63(b) and the corresponding $\{100\}$ pole figure (d) show a preferred orientation of aragonite unit cell $\{100\}$ parallel to the Y-direction. A majority of crystals are aligned in this direction - parallel to the height of the septum. There is approximately a $20^{\circ}$ deviation from the exact $\{100\}$ axis, which may be caused by sampling. Maps (a) and (c), along with the corresponding pole figures indicate that there is a range of orientations about the $\{100\}$ axis.

The top third of the images in Figure 5.63(a-c) represent the prismatic structure in the chamber roof, whilst the lower two thirds of the images represent the fibrillo-lamellar structure found in the chamber floor. From the image in Figure 5.61(a), the porous structure is clearly having a detrimental effect to the indexing in these regions, noted by the small black banded dots in Figure 5.63(a-c). The larger black areas could be due to poor diffraction, 


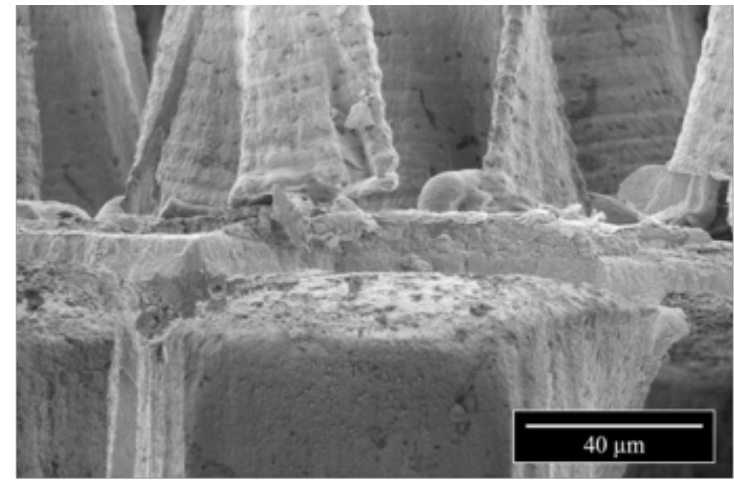

(a)

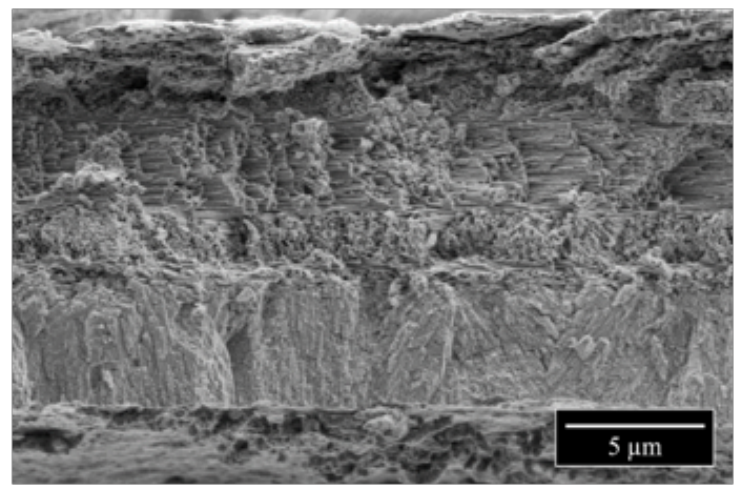

(c)

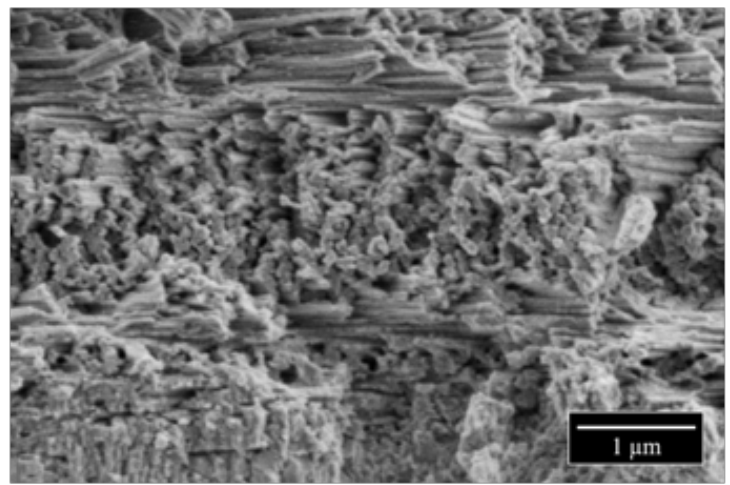

(e)

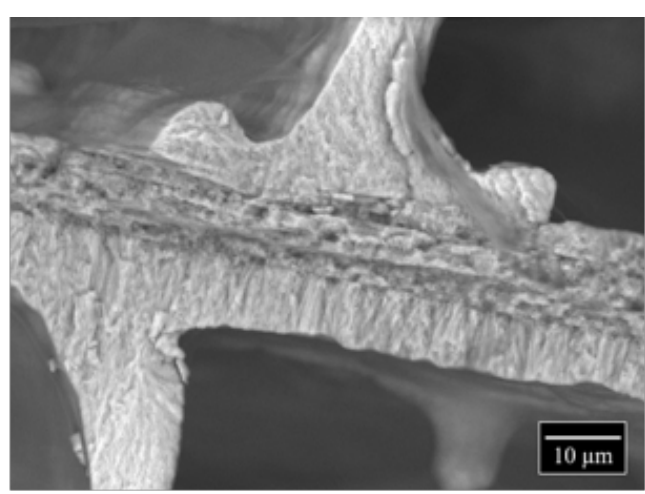

(b)

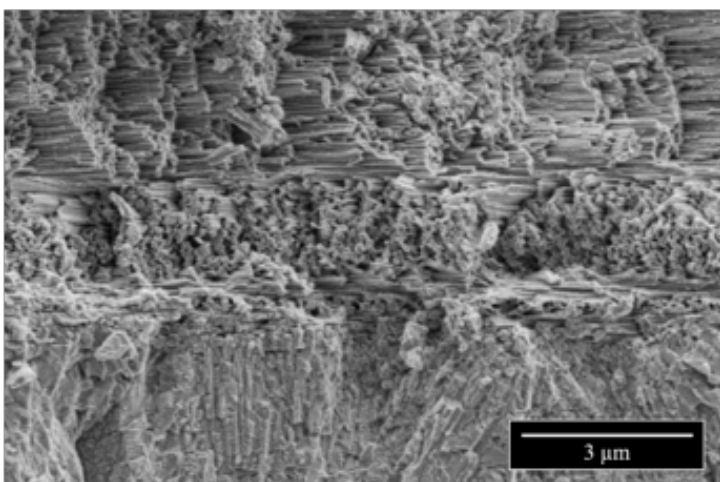

(d)

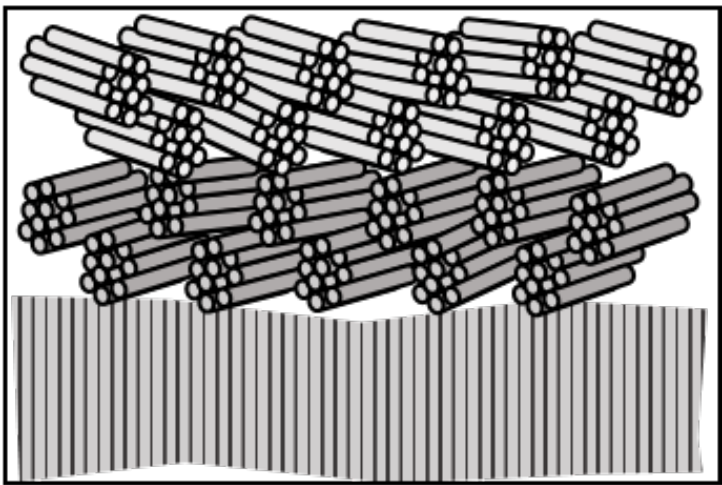

(f)

Figure 5.60: SEM images of septa: (a) pillars intersecting a septum; (b) backscattered image of change of microstructure within a septum and adjoining pillars; (c-d) increasingly higher magnifications of the septum, with the chamber floor and roof having a lamello-fibrillar and prismatic microstructure respectively; (e) increased magnification image of the chamber floor, showing the highly ordered aragonite fibres with different orientations in different planes; (f) a schematic of (e) showing the change in direction of bundles of fibres. Dorsal shield to top of each image. 


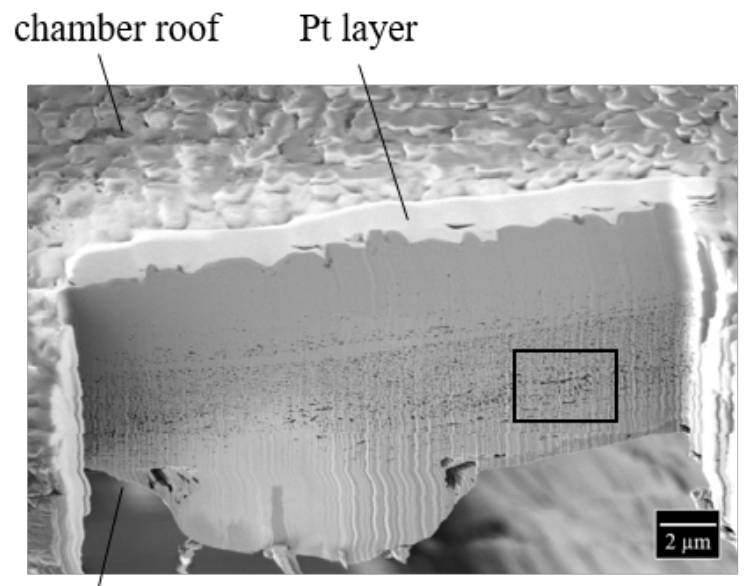

chamber floor

(a)

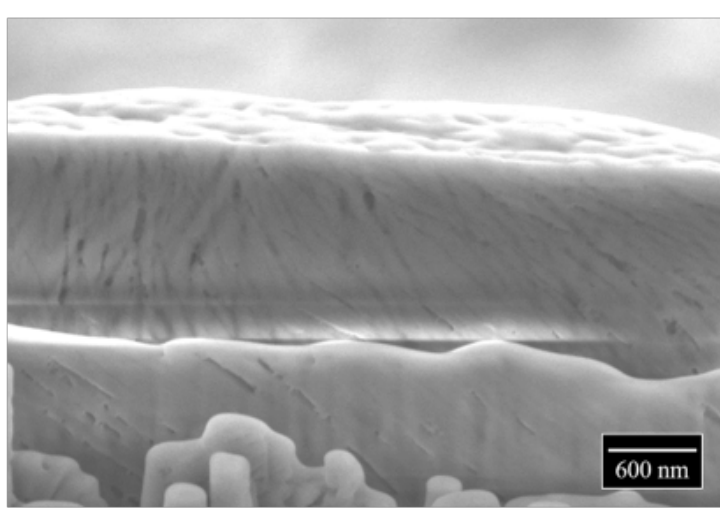

(b)

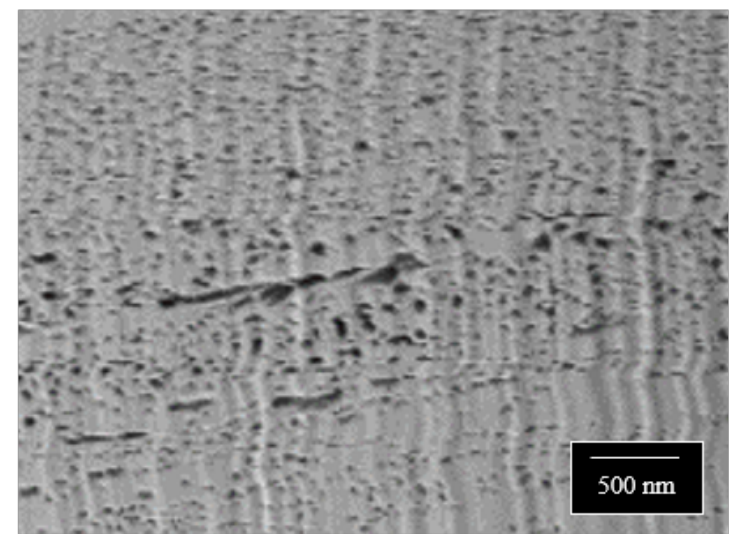

(c)

Figure 5.61: SEM images of a septum that has been sectioned by FIB: (a) in transverse direction (dorsal shield to bottom of image); (b) in the dorsal plane, i.e. at $90^{\circ}$ to (a); (c) zoomed-in region from (a) highlighting porous structure. 


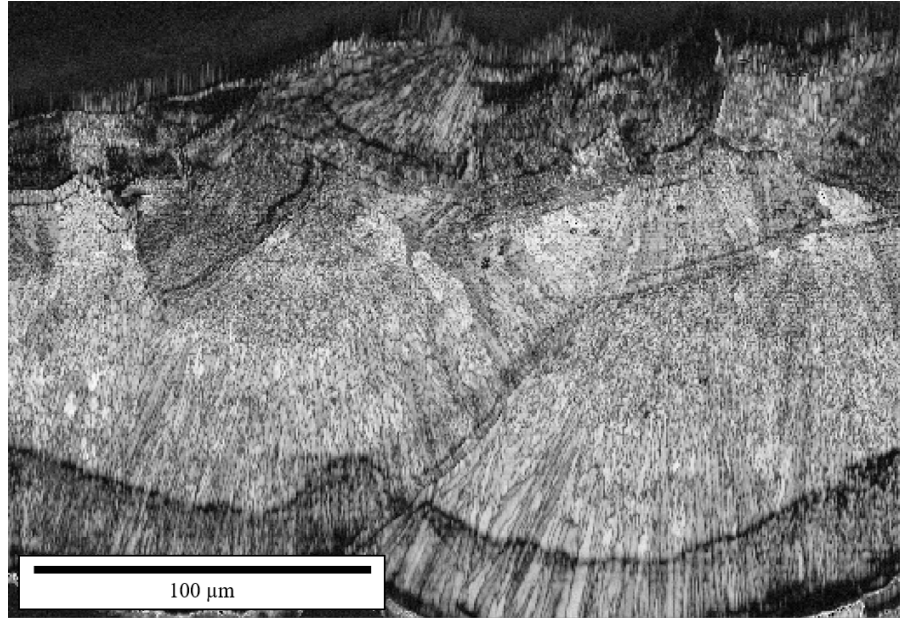

(a)

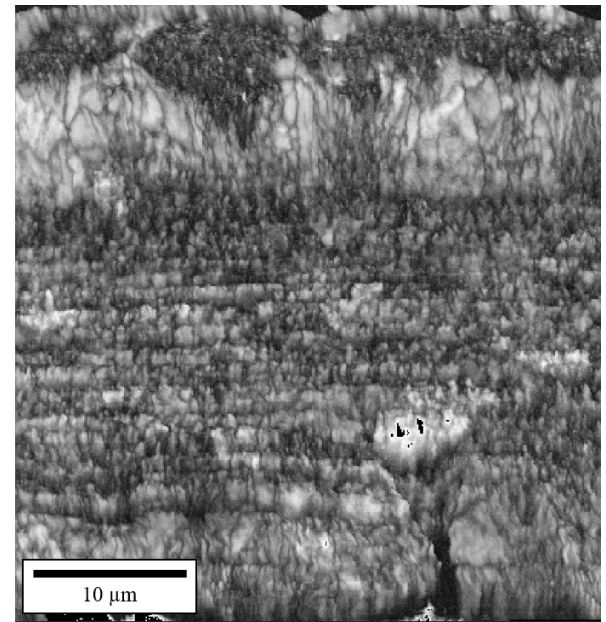

(b)

Figure 5.62: Band contrast maps, showing diffraction intensity: (a) dorsal shield (outer surface to bottom of image); (b) septum (dorsal direction to bottom of image).

from higher amounts of grain boundaries due to smaller crystal size, or higher proportions of organic material, i.e. non-crystalline.

The orientations of the aragonite bundles do not seem to correlate with the in-plane rotations seen in Figure 5.60(e).

The growth of aragonite is preferred along the c-axis $(\{001\})$ relative to other axes [230], therefore it makes sense that the c-axis orientations are in the plane of the septum. As a result, when a new septum is deposited, the aragonite crystals are potentially orientated for the most efficient growth in the longest axes, i.e. laterally and anteroposteriorly across a septum, rather than the height of a septum. This would be indicated by a range of orientations of the $(\{001\})$ and $(\{010\})$ about the $(\{100\})$ parallel to the septa height.

Figure 5.64(b) and the corresponding $\{100\}$ pole figure (d) show a clear preferred orientation in the $\{100\}$ parallel to the Y-direction. The crystals are almost all aligned in this direction - parallel to the thickness of the outer dorsal shield. There is approximately a $15.2^{\circ}$ deviation from the exact $\{100\}$ axis. This is most likely due to sampling, and the curvature of the shield. Maps (a) and (c), along with the $\{001\}$ and $\{010\}$ pole figures indicate that there is a range of orientations about the $\{100\}$ axis.

Contrary to EBSD analysis performed by Cusack and Chung [222] on the dorsal shield, the c-axis of aragonite was not found to be aligned with the long axis of the fibres. Instead, the dorsal shield crystals are orientated in the $\{100\}$ parallel to the shield thickness, with clear rotations about this axis in both the $\{001\}$ and $\{010\}$ directions. The pole figures in Figure 5.64(d) show this.

There were a number of challenges encountered when acquiring EBSD maps of cuttlebone:

- Traditional polishing methods were difficult due to the brittle nature of the lamellar 

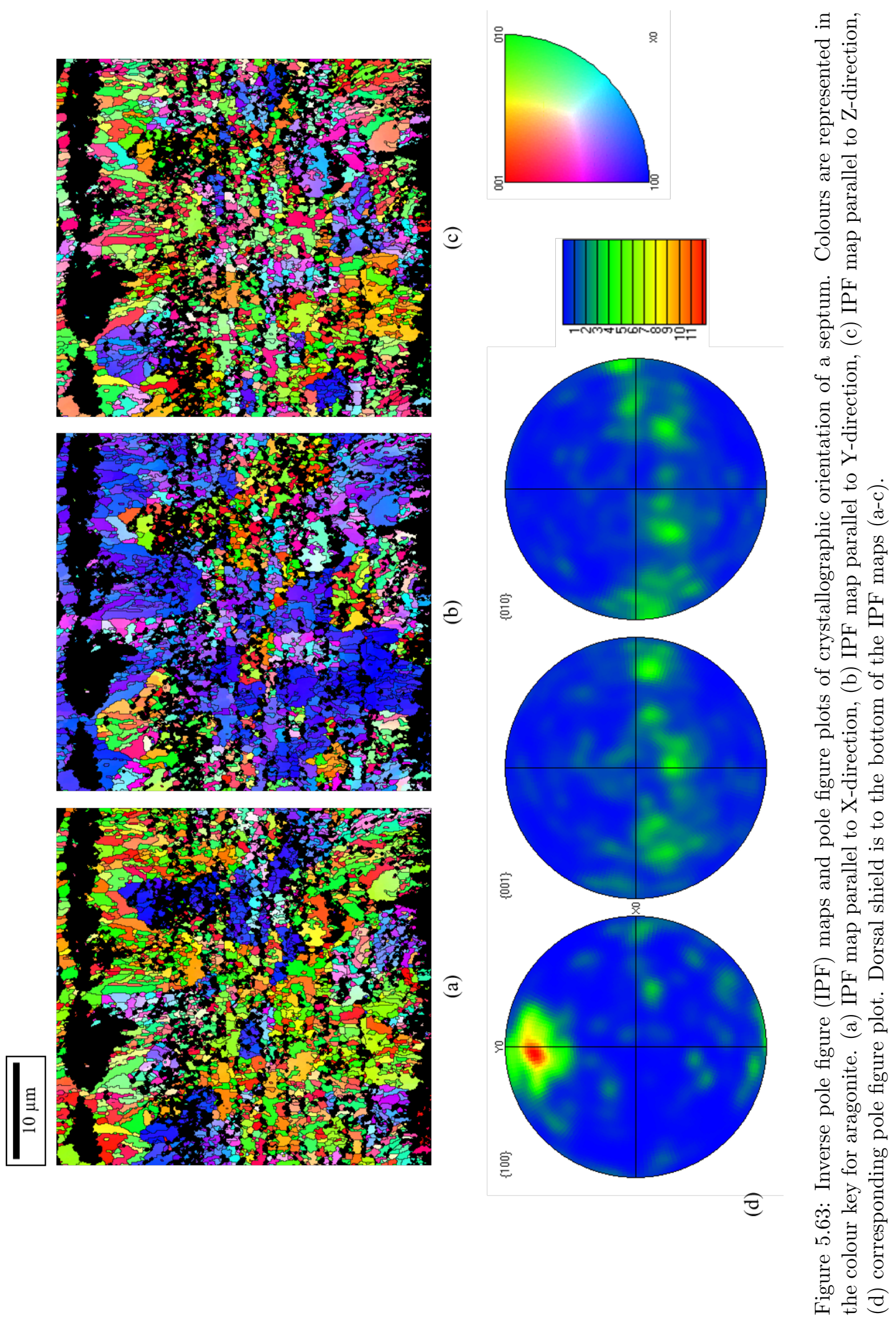


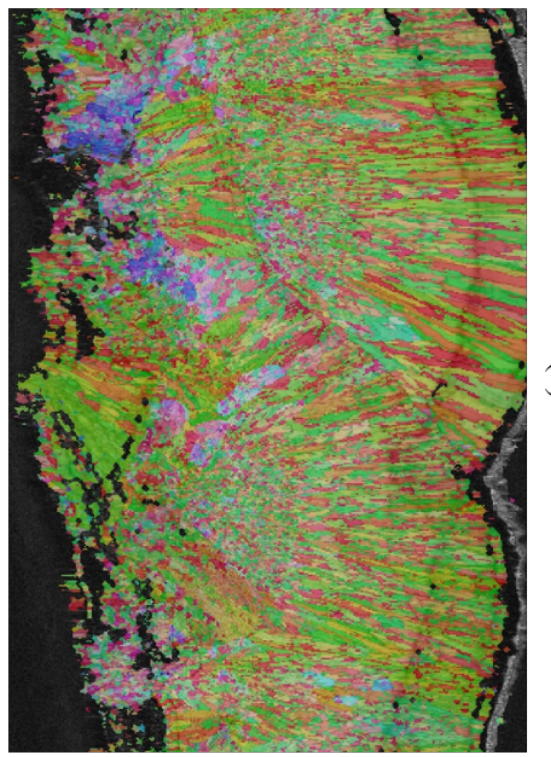

(0)
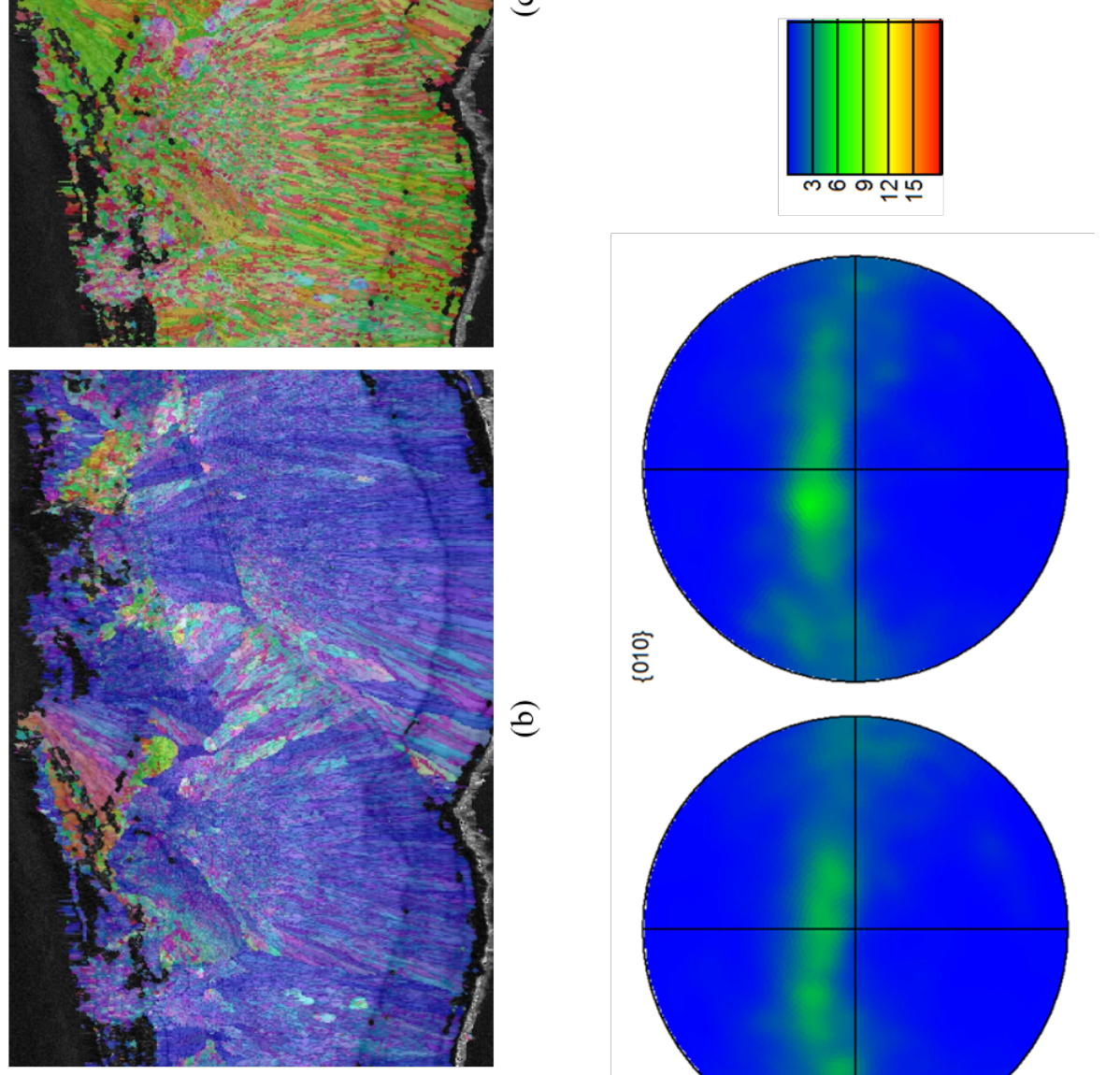

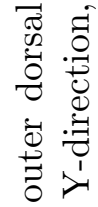

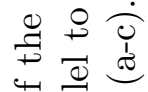

귤

.ำ.

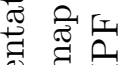

巧

.

룽

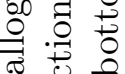

e

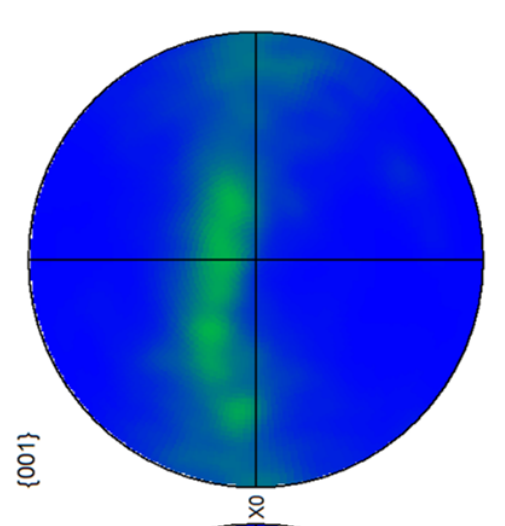

ind

:

$4 x$ 웅

in $ᄋ$. . .

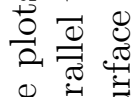

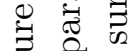

${ }_{5}^{\infty} \infty^{2}$

范

¿ I

령

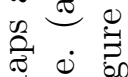

㞼 $\stackrel{0}{.}$

तె

苟

ठํ.

궁 司

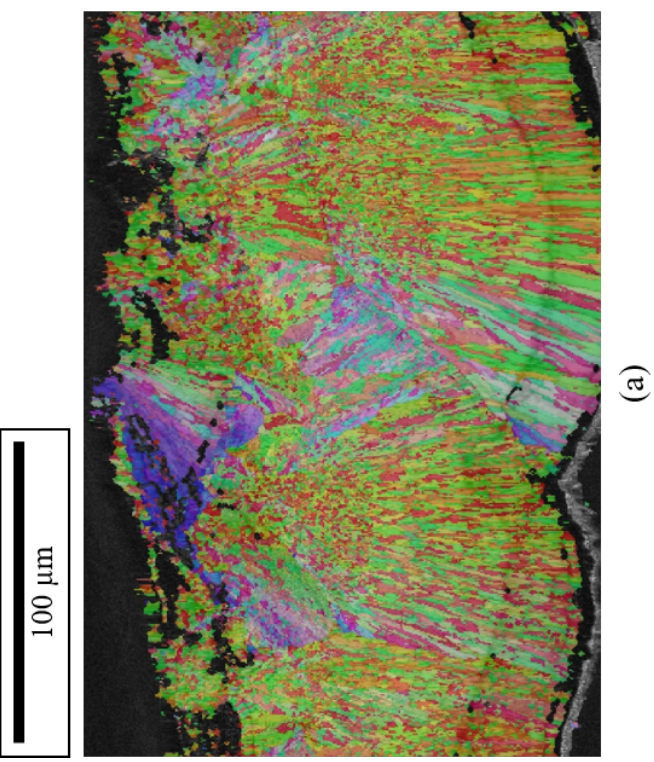

돈류

II 0

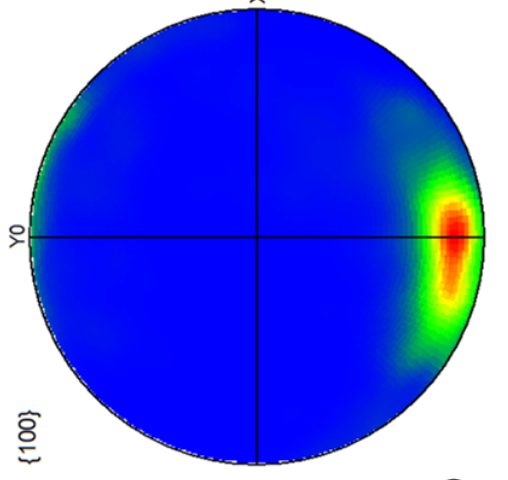

声

范

뭄 坖

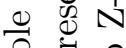

ํ. 융

它式

点芯

- $\quad$ نु

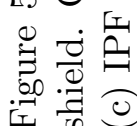


matrix. Cuttlebone was typically softer than the resin it was embedded in. It would grind down at a faster rate than the resin during polishing which would lead to crevices occurring. The result was a sample with topography, therefore unusable for EBSD analysis (BIB and FIB were used to combat this, however these methods are timeconsuming for resulting small areas).

- The porous structure seen in the chamber floor of a septum contributed to poor indexing levels.

- Cuttlebone is a non-conductive sample, therefore it requires carbon coating with an optimum thickness, to reduce charging effects whilst keeping a strong diffraction signal.

- Small feature sizes require a small step size, which increases the time for a map, therefore only small regions are feasible to map.

- As noted previously, the dorsal shield contains approximately 30 - 40\% organic material [177]. This potentially had an effect on the ability to map the inner dorsal shield. The septa have also been identified as having a higher proportion of chitin [181], which would reduce the intensity of diffraction patterns.

Due to the challenging nature of cuttlebone lamellar matrix for EBSD analysis only two maps of relatively small area were produced. Therefore, the results obtained would be strengthened by additional maps from regions of the dorsal shield, septa and pillars.

\subsubsection{Chemical analysis}

As structural changes have been identified, it follows to analyse the chemical distribution across regions investigated in the previous section.

\subsubsection{Electron Dispersive X-ray Spectroscopy}

EDS analysis was carried out on regions of both the dorsal shield and lamellar matrix to monitor any changes in chemistry across the cuttlebone. From previous microstructural analysis and BSE imaging, the dorsal shield was identified as having a band of reduced atomic density. Microstructural changes were also observed both within an individual septum and between the septa and pillars.

EDS maps and spectra were collected from regions of the dorsal shield, septa and pillars. The darker band of green on the right hand side of Figure 5.65 displays the reduction in calcium in the inner region of the dorsal shield.

Three smaller areas, indicated by Map2, Map3 and Map4 in Figure 5.65, were chosen based on their location within distinct regions on the EDS map to calculate the weight percentage of calcium. Map2 in the large higher calcium region on the outer dorsal shield had a calcium weight of $46.7 \%$, Map3 in the dark green band had a calcium weight of $45.3 \%$ 
and Map4 in the innermost portion of the dorsal shield at the interface with the lamellar matrix had a calcium weight of $46.0 \%$.

EDS mapping was also performed on multiple areas of the lamellar matrix, i.e. pillars, septa, and the interface between pillar and septum. No significant changes in chemical composition were seen. This is in contrast to chemical analysis using micro-Raman spectroscopy performed by Florek et al. [181], where the pillars were found to contain more aragonite (polymorph of $\mathrm{CaCO}_{3}$ ) than the septa. This may not be evident using EDS due to the low sensitivity required to determine any changes in these regions.

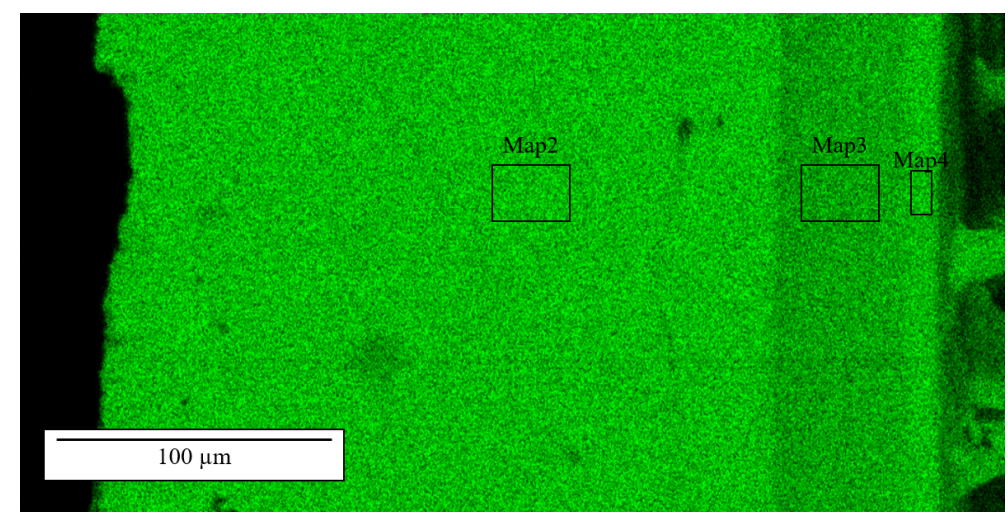

Figure 5.65: EDS map of Ca distribution in the dorsal shield. Boxes depict mapped areas for weight percentage calculations.

This band of less calcified material is known to reduce in size dependent on its location within the dorsal shield [229]. Regions directly above chambers, like the sample in Figure 5.65 , are typically thicker with calcified material, whereas at the lateral edges of the dorsal shield, the organic band is typically a lot thicker. In the example in Figure 5.65, the reduction in calcium was found to be $1.4 \%$ compared to the outer dorsal shield and $0.7 \%$ compared to the innermost portion of the dorsal shield.

\subsubsection{XPS}

XPS spectra and maps were collected from three regions of the cuttlebone. Sample s2 is taken from the centre of the cross-section whereas s3 and sh1 are taken from opposing lateral edges. Stoichiometry and 2D distributions of these areas from a transverse crosssection were acquired. XPS analysis was used in parallel with EDS due to its additional surface sensitivity and quantitative capacity.

Table 5.10 gives the elemental composition percentage of the regions shown in Figure 5.66. These compositions are taken directly from the spectra, an example of which is shown in Figure 5.67. All three samples show composition percentages in the same order of magnitude for each element. Sample sh1 has the highest percentage of calcium, almost three times as much as sample s2, whilst sample s3 has almost twice as much as s2. sh1 also has the 


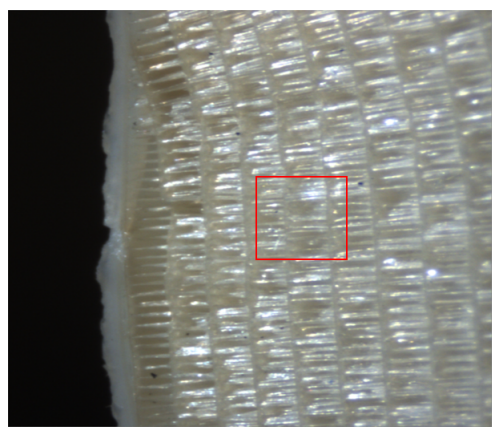

(a)

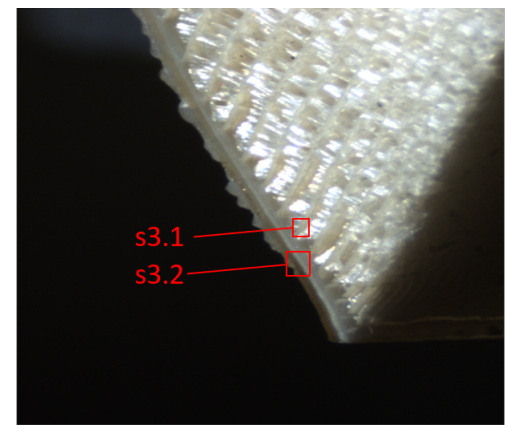

(b)

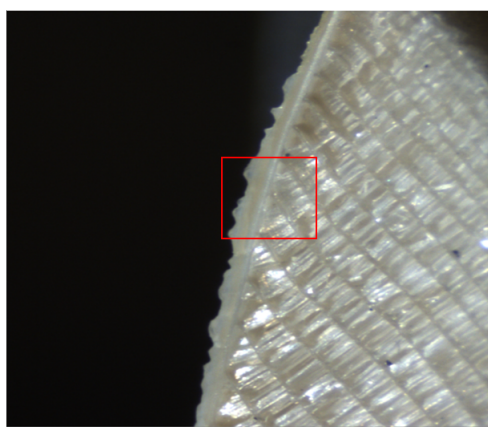

(c)

Figure 5.66: Samples used for XPS analysis: (a) s2; (b) s3; (c) sh1. Each image measures $7 \times 6 \mathrm{~mm}$. Boxes display regions mapped in Figure 5.68.

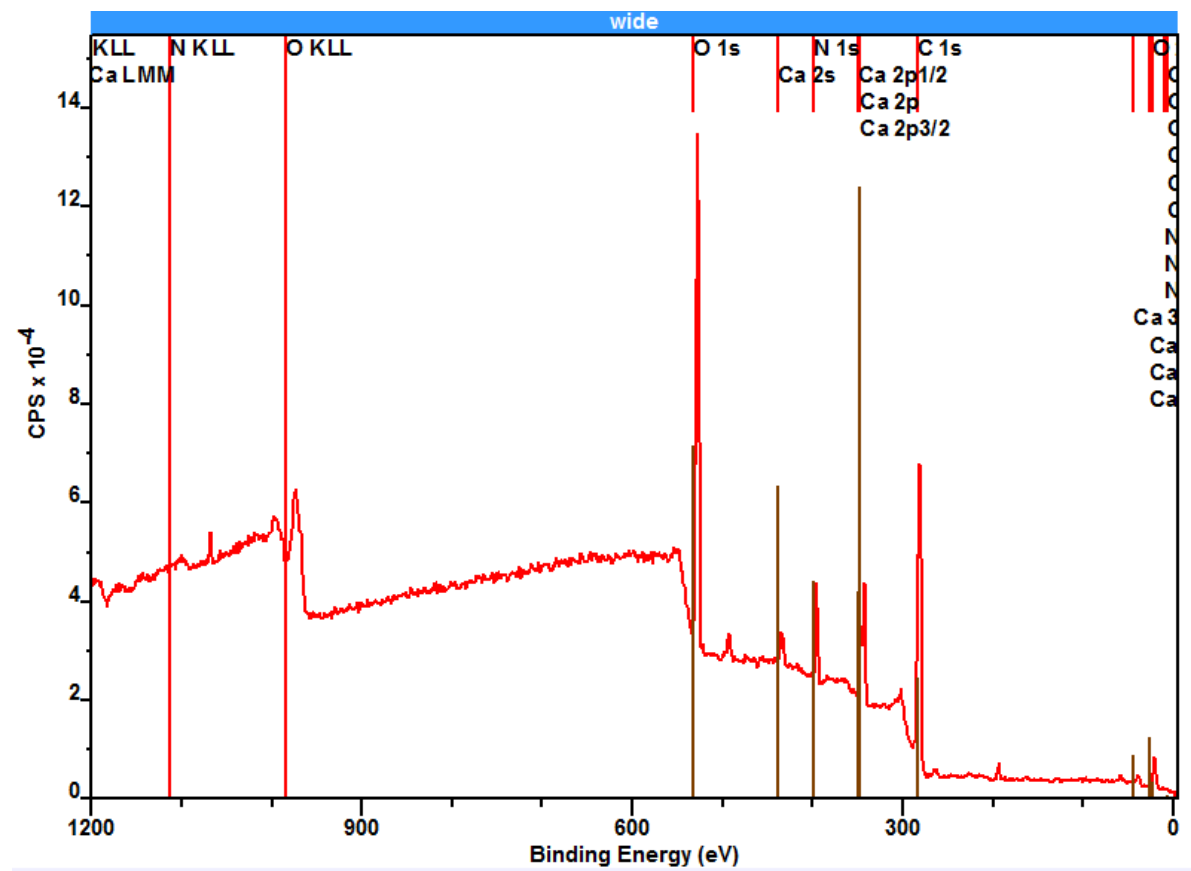

Figure 5.67: Elemental wide spectrum of cuttlebone with annotated peaks shown in Table 5.10 . 
Table 5.10: Region element composition \% acquired by XPS analysis.

\begin{tabular}{lrrrrc} 
& \multicolumn{5}{c}{ Composition (\%) } \\
Region & C 1s & N 1s & O 1s & Ca 2p & Si 2p \\
\hline s2 & 64.67 & 7.86 & 23.86 & 2.18 & 1.43 \\
s3 & 68.68 & 5.38 & 21.73 & 4.21 & - \\
sh1 & 63.29 & 3.54 & 27.25 & 5.92 & - \\
\hline
\end{tabular}

highest amount of oxygen, but the lowest amounts of nitrogen and carbon. It is unclear why no composition percentages are recorded for silicon for s3 and sh1. Perhaps the threshold amount was insufficient to be recorded. The organic component of the cuttlebone is likely to vary across a specimen, hence the fluctuation in the compositions given. However, the use of additional maps of the 2D elemental distributions help to identify the compositions across the cuttlebone.

Maps were acquired for carbon, calcium, oxygen and nitrogen for a range of regions. Some of these are shown in Figure 5.68. Images (a) and (b) from sample s2 show the calcium and carbon distributions respectively. This sample shows a higher distribution of calcium in the septa than the pillars, whereas the inverse is seen for carbon. Image (c) taken from sample s3.1 shows the sample pattern as (a) for calcium. The septum is the bright pink band running diagonally towards the top right corner of the image. The less bright pink, less calcium, region above the black triangle is a pillar. Images (d) and (e) from sample s3.2 show calcium and carbon distributions respectively across the dorsal shield. The darker band in (d) is a reduced band of calcium. This reduced calcium band in the dorsal shield has also been seen in EDS analysis and BSE imaging. However the thickness and position relative to the outer dorsal shield surface is different. This indicates that regions towards the longitudinal axis are different to those at the lateral edges. In addition to this, (e) shows a reduced band of carbon between the calcium band and the dorsal shield edge. Image (f) shows the calcium distribution in sample sh1. This sample appears to have a substantial crack within the dorsal shield. This is shown by the black band running almost vertically. However the reduced calcium band in the shield is still clearly evident. Again, more calcium is noted in the septum compared to the pillars. The black triangles in (c) and (f) are the gaps at the intersection of the lamellar matrix and dorsal shield also seen in Figures 5.56 and 5.58 .

There were no discernible differences across the sampled regions for oxygen and nitrogen. There is the possibility that topography of samples may cause shadowing which could impact on the accuracy of the imaging. All maps in Figure 5.68 are $256 \times 256$ pixels. Therefore depending on the scale, the accuracy of the imaging may be affected. This accounts for the 'spreading' seen in the images.

In Florek et al. [181], micro-Raman maps conducted on a septum show the richest aragonite areas are located along the pillars with the poorest regions along the septa, hence 


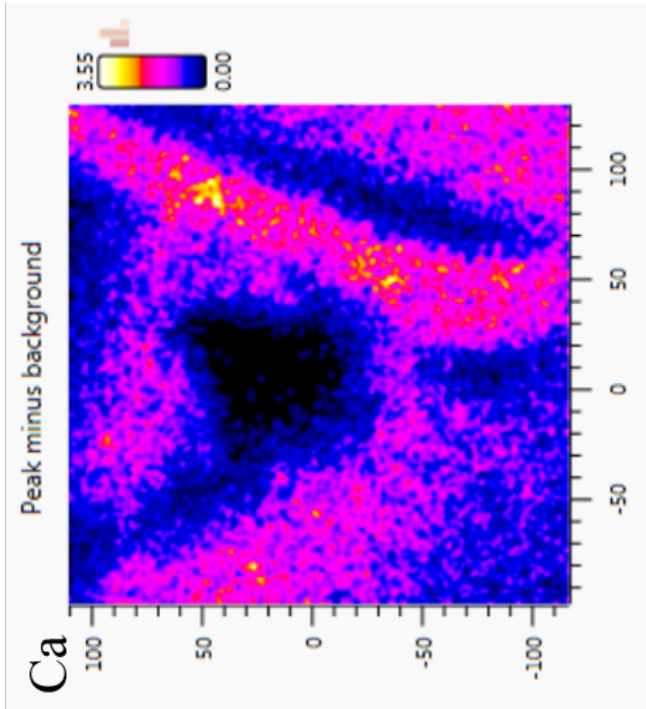

wrt
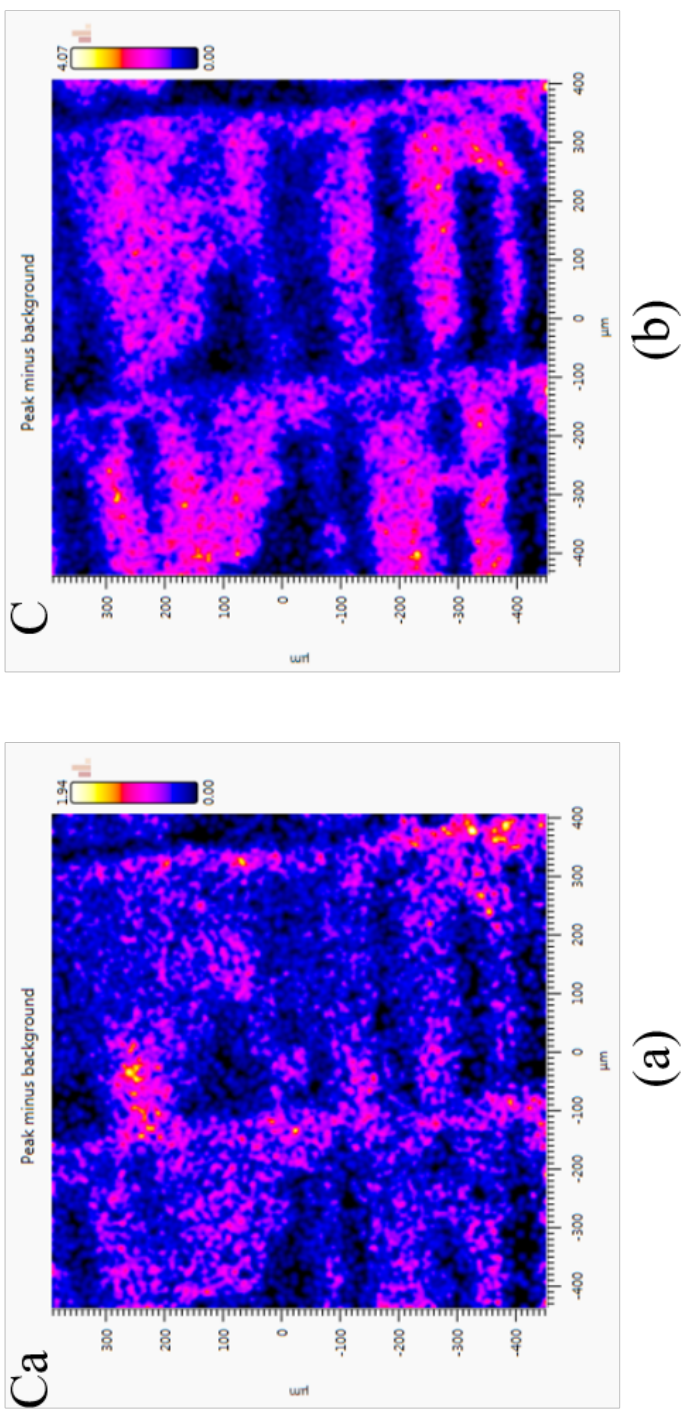
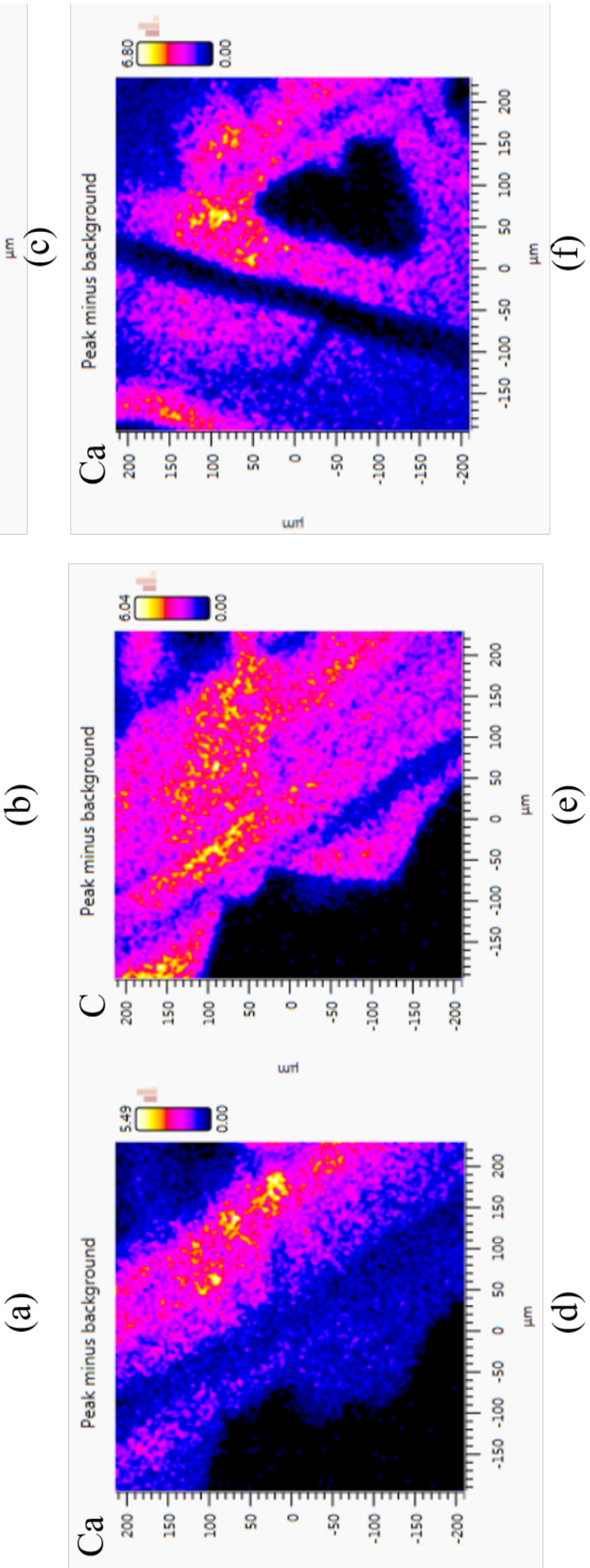

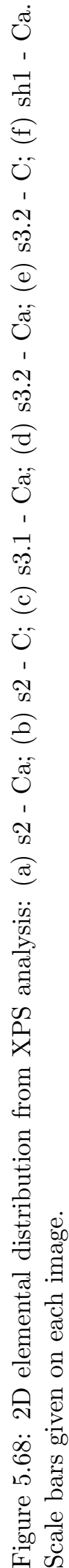


indicating septa are less calcified. It was also shown that septa are richer in chitin than pillars. This contrasts with the analysis performed, which shows that regions richer in calcium were found to be the septa.

\subsubsection{Mechanical analysis}

Further to the structural and chemical investigations, the mechanical properties and characteristics of the cuttlebone were determined using nanoindentation and time-lapse imaging under compressive loading.

\subsubsection{Nanoindentation}

Nanoindentation testing was used to identify the material properties of the different structural components. Nanoindentation was conducted along polished cross-sections of the dorsal shield, pillars and septa.

Table 5.11: Reduced elastic modulus and hardness measurements obtained from nanoindentation testing.

\begin{tabular}{lrr} 
Sample area & $\begin{array}{r}\text { Elastic modulus, } E \\
(\mathrm{GPa})\end{array}$ & $\begin{array}{r}\text { Hardness, } H \\
(\mathrm{GPa})\end{array}$ \\
\hline Dorsal shield (outer) & $63.20 \pm 5.31$ & $3.73 \pm 0.48$ \\
Dorsal shield (inner) & $23.38 \pm 9.23$ & $0.78 \pm 0.59$ \\
Pillars & $51.00 \pm 8.15$ & $4.40 \pm 0.74$ \\
Septa & $29.60 \pm 8.30$ & $1.90 \pm 1.10$ \\
\hline
\end{tabular}

Table 5.11 shows the mean reduced elastic modulus $(E)$ and hardness $(H)$ measurements obtained from nanoindentation testing. The mean reduced elastic modulus of the outer shield is $63.20 \mathrm{GPa}$, almost three times that of the inner shield (23.38 GPa). This relationship is also seen in the mean hardness values, with the outer shield (3.73 GPa) being almost 5 times that of the inner shield $(0.78 \mathrm{GPa})$. The mean reduced elastic modulus and hardness of the pillars (51.00 $\mathrm{GPa}$ and $4.40 \mathrm{GPa}$ respectively) were comparable with the outer shield and roughly twice the septa values (29.60 GPa and 1.90 GPa respectively). The biggest variation in hardness is found in the septa (standard deviation of 1.10). The measured values for dorsal shield show an abrupt transition from the outer to the inner layers, i.e. a gradient was not identified at this interface (based on indents a few microns apart).

An increase in the amount of organic material is evident in the values of reduced elastic modulus and hardness for the inner dorsal shield and septa. Reduced calcium content has been seen in the dorsal shield, see Figures 5.65 and 5.68, for instance. Reduced mineralisation in the septa has not been seen in the chemical analysis in this study, however it has been analysed before by Florek et al. [181]. They found higher proportions of aragonite and 
chitin in the pillars and septa respectively. Their results also support the suggestion from the data in Table 5.11, that the inner dorsal shield and septa show similar levels of mineralisation, whereas the outer dorsal shield and pillars have higher levels of mineralisation and are more similar in composition.

As mentioned previously, the proportion of organic material varies depending on the sampled region of the cuttlebone, hence the differences shown in the samples used for nanoindentation and chemical analysis. Considerable change in elastic modulus was seen across the dorsal shield, compared with the small weight percentage difference noted in section 5.3.2. However other work has shown that there can be a large spread in elastic modulus for a large range of mineral volume fractions [231].

A change in material properties was not identified across an individual septum, where a change in microstructure was clearly evident (see Figure 5.60). This is due to the limitations imposed by the sample septa height and corresponding edge effects encountered with thin structures. From the EBSD maps (Figure 5.63), the crystallographic orientation of aragonite in the septum shows a preferred orientation, which may result in the material properties remaining equivalent. However, this cannot be verified due to limited indentation values across an individual septum and regions analysed with EBSD.

The cuttlebone is a complex composite material of varying morphology. Characterising the material properties of these individual components and relating these to their structures may help to inform the design of cellular materials based on a cuttlebone-like architecture. This relationship may also provide insight for the development of synthetic scaffolds, where the bulk material properties of scaffolds are compared to those of cuttlebone [232].

\subsubsection{Compression testing and time-lapse imaging}

Sections of cuttlebone were loaded progressively and a series of 3D images were obtained using X-ray microtomography. This produced a time-lapse series allowing structural deformation to be visualised. Load $(\mathrm{N})$ and extension $(\mathrm{mm})$ data were recorded at an extension rate of $0.25 \mathrm{~mm} / \mathrm{min}$. Scans were conducted in between each compressive loading stage. Loading was removed when local maximum loads had occurred. A load-displacement curve was used instead of a traditional stress-strain curve because the cross-sectional area used to calculate stress is not equivalent to the area of the pillars in contact with the compression platens.

The corresponding load-displacement curve is given in Figure 5.69. The curve is coloured according to the relevant stage of loading. Each stage shows a similar pattern of linearly increasing displacement with load, until a peak load is reached and followed by a slight decrease in load. There are often short plateau regions after a peak load where displacement increases and the load remains approximately constant. This indicates that the cuttlebone chambers are undergoing localised compaction via buckling of pillars, which in turn leads to a progressive collapse of the chambers. Figures 5.70 and 5.71 help to illustrate this. Inspection of the $3 \mathrm{D}$ renders shows that chamber failure correlates with load maxima in 
each load stage. For example, in load 5 (dark blue curve), there are two maxima, and the $3 \mathrm{D}$ render shows that the bottom 2 chambers have failed. In some instances, chambers that already show some level of damage, like those flagged with a white arrow in the reference 3D render in Figure 5.70, become fully compressed before other chambers are affected. These chambers are shown to be fully compacted in the Load 2 3D render.

The results show a progressive collapse mechanism. This is in agreement with the work conducted by Gower and Vincent [191]. They showed that critical compressive stress varied along the longitudinal axis, from $1.03 \mathrm{MPa}$ at the posterior end to $2.1 \mathrm{MPa}$ at the anterior end. These findings were not however correlated with 3D imaging, as done in this study.

One of the principle functions of the cuttlebone is to reduce the risk of implosion, due to hydrostatic pressures at depth. The progressive collapse of chambers seen in the results is beneficial to the cuttlefish as it has been shown that they can survive multiple chamber failures without catastrophic effects [186]. It has been suggested that the meandering of pillars contributes to compressive stability as it reduces the likelihood of pillar buckling by maximizing their second moment of area [177]. Figure 5.50 shows that, from the quantitative analysis performed on pillar morphology, average tortuosity changes substantially across the cuttlebone. Figure 5.50 and the results in Figures 5.38 - 5.44 show that tortuosity changes both within a chamber and throughout the cuttlebone, in the dorsoventral, transverse and longitudinal axes. This suggests that the level of compressive stability of pillars will vary throughout the cuttlebone, presumably with the most amount of compressive stability occurring in the most tortuous regions. Regions of cuttlebone which regularly fill with cameral fluid to regulate buoyancy typically have mid-levels of tortuosity, as well as being relatively thin, and having low Feret lengths. The combination of these features would make these pillars more vulnerable to failure, however these regions are also supported by the fluid, which may help to resist implosion [186]. If this is indeed the case, the cuttlebone may have evolved over time to deposit less material in these regions, therefore reducing energy expenditure and material resources.

Whilst performing mechanical tests on natural materials, differences in experimental results are expected due to variations in the microstructure and potential defects. Irregularities may include: structural differences due to sampling regions; age and habitat of specimen; defects, both natural and those introduced by sample preparation; and natural variation, for example, septal curvature and thickness, and amount of organic material. In order to overcome this and compare mechanical data, a statistical analysis would be used. However, in this case, visualisation of the damage mechanisms was the principal desired outcome rather than the validation of the previous findings.

The pattern of failure displayed by the cuttlebone is typical of a brittle foam [233], and more generally a cellular solid [48] as it displays linear elastic, plateau deformation and densification mechanical behaviours. The novel architecture of cuttlebone shows promise for energy absorbing applications, due to its progressive failure mechanism.

A more sophisticated technique for the observation of structural deformation is required 


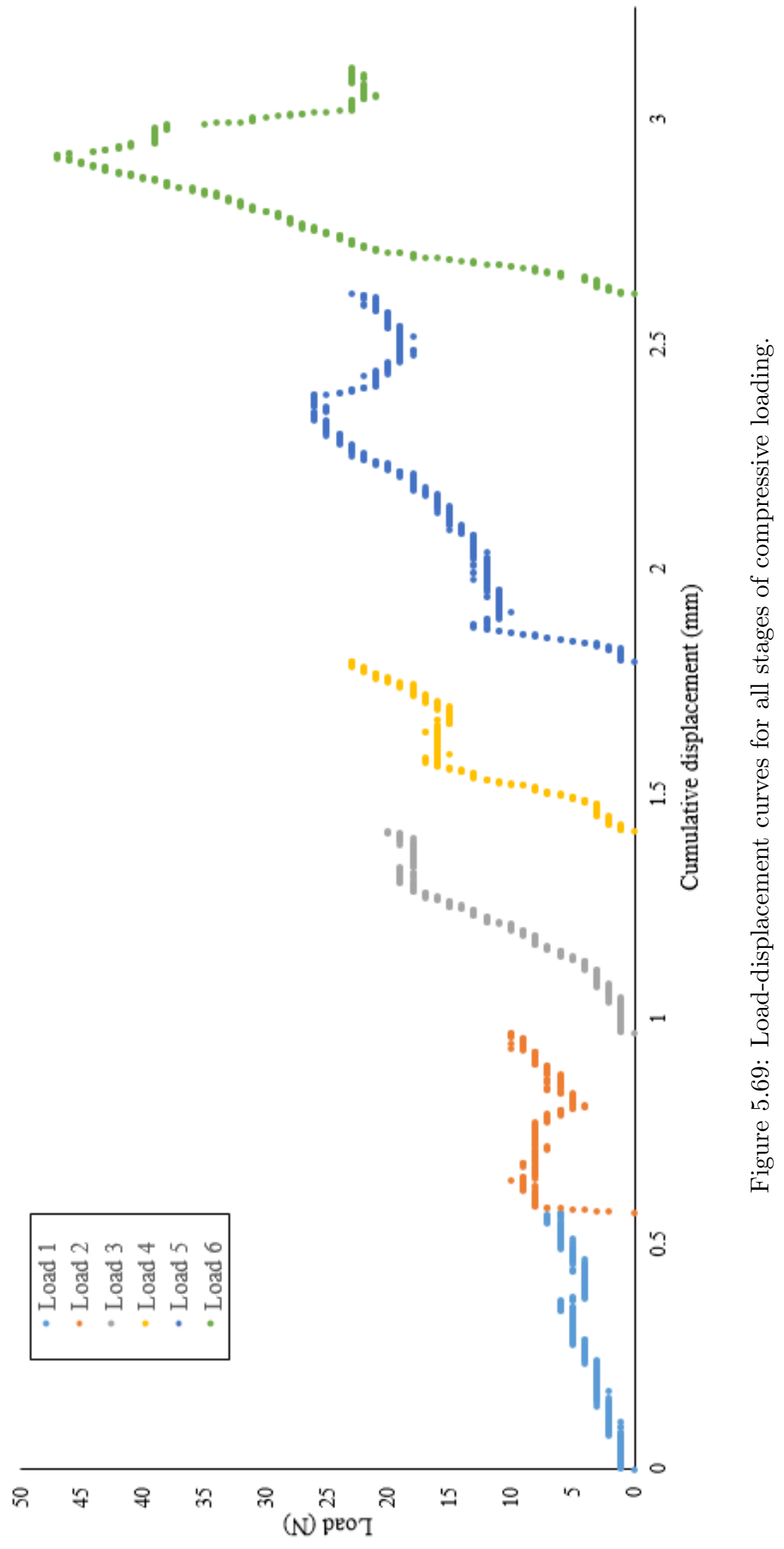




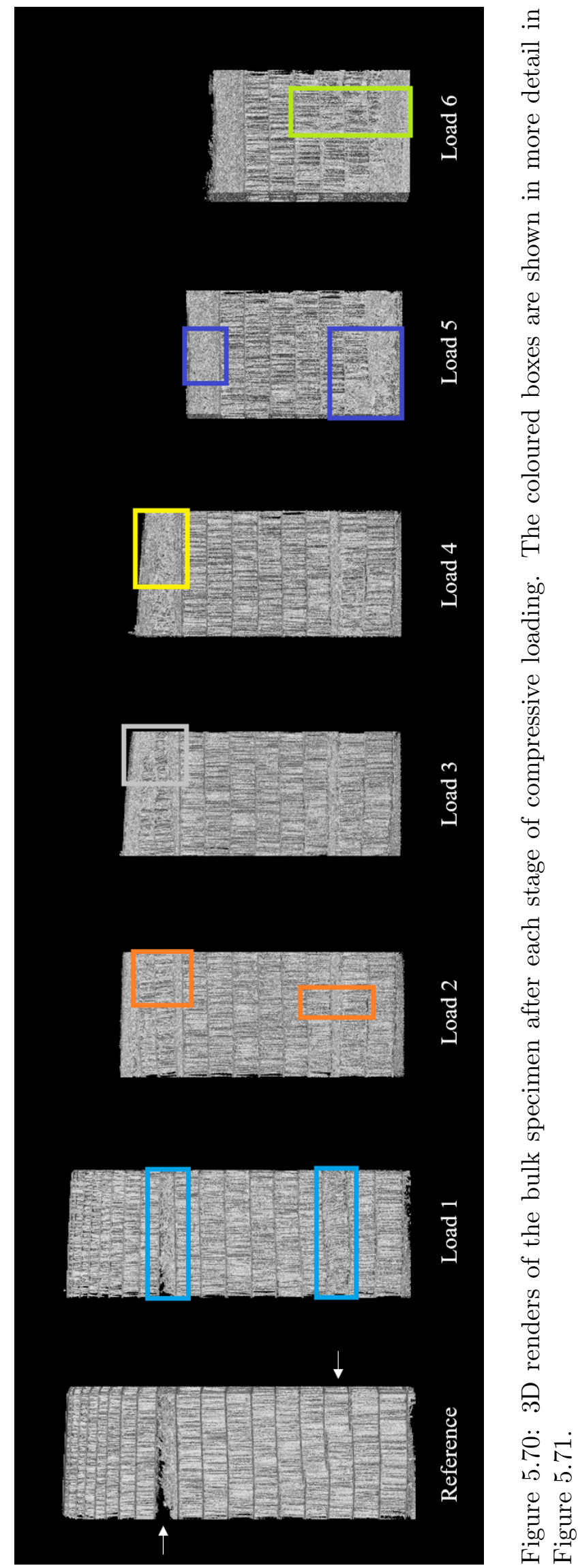




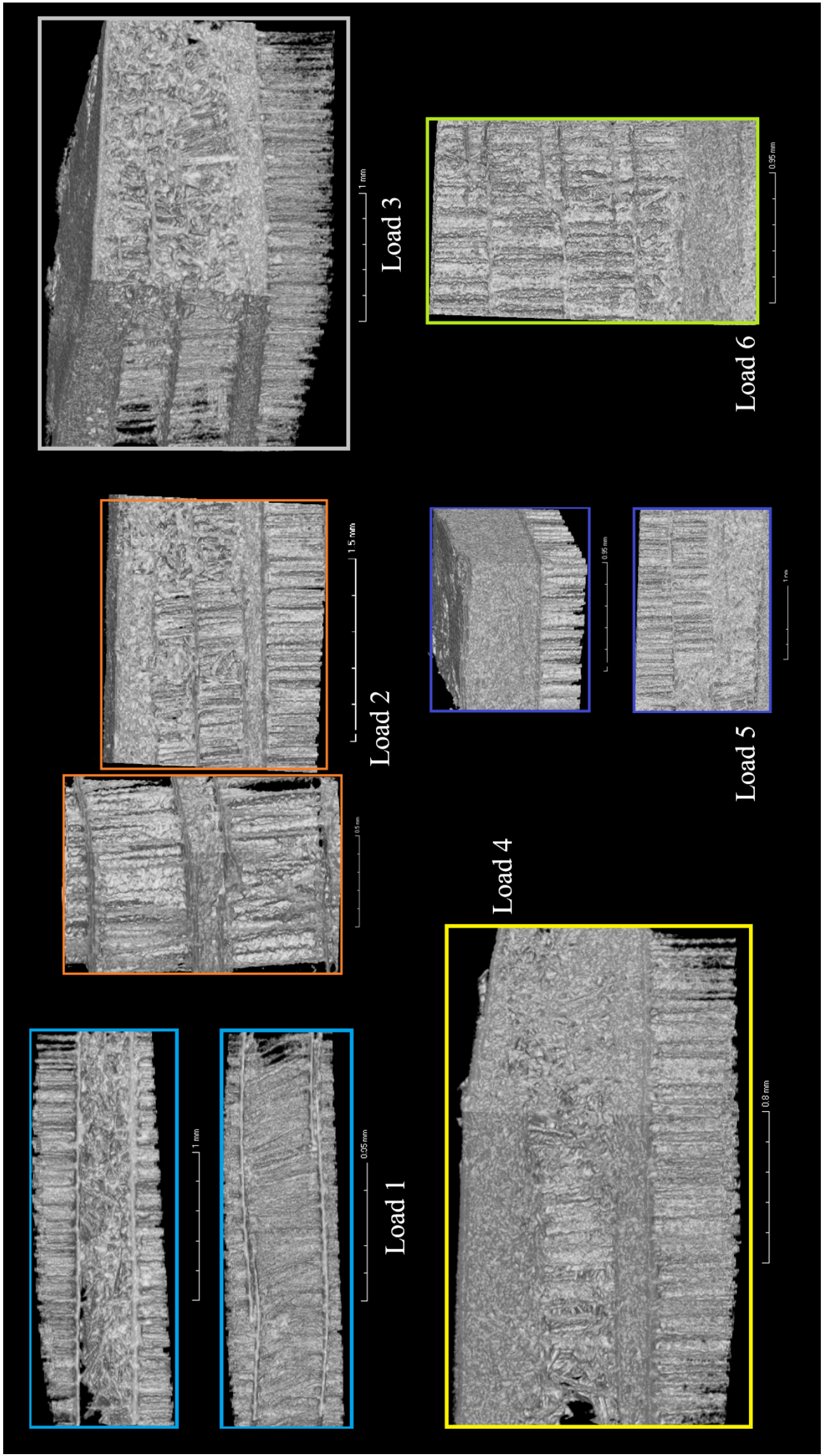

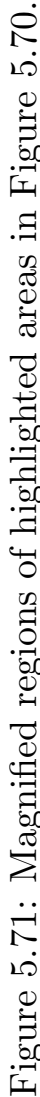


to reduce any deformation occurring throughout the transportation of samples from the Xray $\mathrm{CT}$ scanner to the compression rig, and the removal and reapplication of the compressive loading for each load stage. An in-situ compression/tensile stage for $\mu \mathrm{CT}$ would overcome both of these challenges.

The use of time-lapse imaging provides insight into the failure behaviour of materials. It highlights the compression behaviour of cuttlebone, however in order to investigate single chamber failure, for example, where a pillar buckles first, higher scan resolution and smaller strain increments would be required as well as accompanying quantitative analysis for strain distribution (i.e. digital volume correlation).

The focus of this compression testing and time-lapse imaging was to visualise the failure mechanism of the cuttlebone. The following section explores and compares the behaviour under compression of different regions.

\subsubsection{Compression testing of additive manufactured structures}

The aim of this work was to use 3D printing technology in aiding and understanding the effects of pillar geometry on resistance to compressive forces. 3D printed structures representing cuttlebone geometry were loaded under compression to attempt to link form and function. Load and extension data was recorded at the rates given in Table 5.7. Loading was removed when the structures had clearly failed. For the single pillar and single chamber structures, stress-strain curves were plotted and the cross-sectional area was taken at midpoint. For the three-chambered structures, a load-displacement curve was used as the cross-sectional area of the cuttlebone was not equivalent across the chambers.

Figure 5.72 displays stress-strain curves for three samples of filleted and non-filleted pillars. The curves show that for most of the samples, there are no clear differences in the peak compressive strength. However, there are clear differences between filleted and nonfilleted pillars when loading is continued after initial failure. Inspection of the failed pillars shows that samples without filleting have fractured at the floor and ceiling as well as the midpoint of the pillar. This is likely due to the stress concentration effects of sharp corners at the extremes of the pillars. Those with filleting instead show buckling of the pillars only at the midpoint with compaction of the pillars under increasing load.

Figure 5.73 displays stress-strain curves for four single chamber samples of varying pillar morphologies. cham2 records the highest amount of compressive strength and a sharp reduction in strength once initial failure has occurred. cham 3 has the next highest amount of compressive strength, but displays a very different stress-strain curve in that the chamber loses very little compressive strength once initial failure has occurred, indicating compaction of the chamber. cham 1 and cham 4 record similar amounts of maximum compressive strength but have different stress-strain curves.

All pillars buckle at the midpoint of the cross-section, however the pillars with low tortuosity and high directionality (cham2 and cham4) demonstrate shear failure between layers (septa) in a direction perpendicular to the length of the pillars. cham3 has pillars 


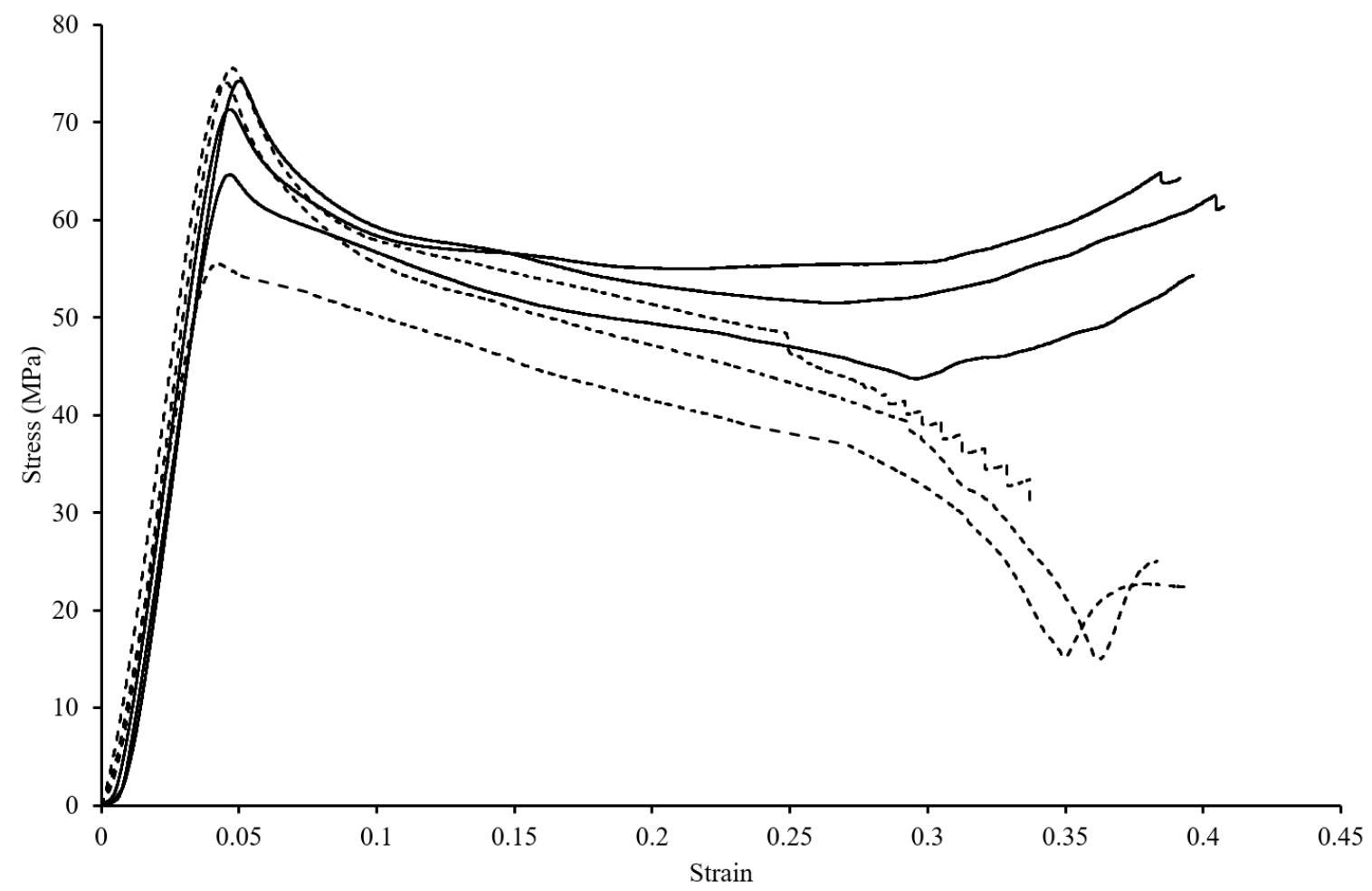

Figure 5.72: Stress strain curves for all single pillars. Filleted pillar data represented by solid lines, non-filleted pillar data represented by dashes. 
with higher tortuosity and these show a stress-strain curve more typical of a cellular structure with a linear elastic region, plateau deformation and densification. cham1 shows behaviour in between these two failure modes. The different failure mechanisms can clearly be seen in Figure 5.74, which displays before and after photos of cham2 and cham3.

Figure 5.75 displays load-displacement curves for a three chamber cuttlebone structure and a three layer honeycomb structure. The three layer cuttlebone structure demonstrates the progressive collapse mechanism identified in section 5.3.3.2. From the plot, failure of the separate chambers can be linked to the individual peaks in the load-displacement curve. This curve follows a typical cellular solid failure mechanism as mentioned previously. The honeycomb structure starts to fail at a slightly higher load and after more displacement. From here the failure is gradual as the honeycomb core buckles. Under observation, the honeycomb fails simultaneously across all layers, rather than in isolated layers like the cuttlebone. Figure 5.76 displays before and after photos of the three layer cuttlebone and honeycomb structures, the differing failure modes are visible in the failed structure images. The amount of energy absorbed can be approximated as the area under the curves in Figure 5.75. The honeycomb structure is able to absorb approximately twice the amount of energy as the cuttlebone structure before they initially fail, however over the total displacement the cuttlebone absorbs marginally less energy. This supports the potential of the progressive failure mechanism providing energy absorption capabilities.

The root-like pillars are shown to impact the position of pillar failure as the stress is concentrated at the midpoint of the pillar rather than at the chamber floor and ceiling. This would help to avoid fractures at septa. Any damage to septa could result in the transfer of fluid between chambers, therefore reducing ability of the cuttlefish to regulate buoyancy effectively [186].

The initial results in the single chamber compression tests help to support the idea that tortuosity of pillars contributes towards compressive stability [177]. cham2 appears to demonstrate shear failure between septa under compression, however these regions of low tortuosity are found in close proximity to the dorsal shield, which may help to mitigate the risk of this occurring due to high rigidity of the shield [213]. cham4 also displays shear failure between septa. This chamber is located in the posterior region, which is an area that regularly fills with cameral fluid to regulate buoyancy, and so the chamber may also be supported by fluid which could help to resist pillar failure [186].

A limitation of this 3D printing approach is that it does not allow for the same material composition or length scales as those found in cuttlebone. The simplified approach demonstrated here could be substantially improved by the addition of multi-material 3D printing, which would allow changes in material properties between structural components to be considered. This would also allow the role of the organic matrix as a toughening mechanism to be investigated. As noted in [234], the differences in indentation modulus in the structural components between pillars and septa may represent a toughening mechanism. This is not captured in this study therefore further work would be required to include these changes in 

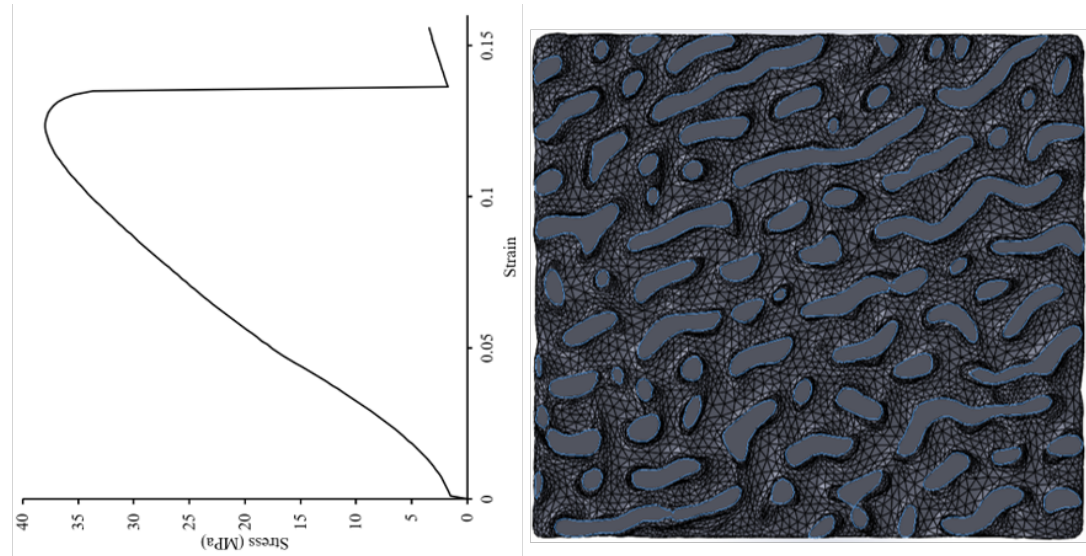

悫
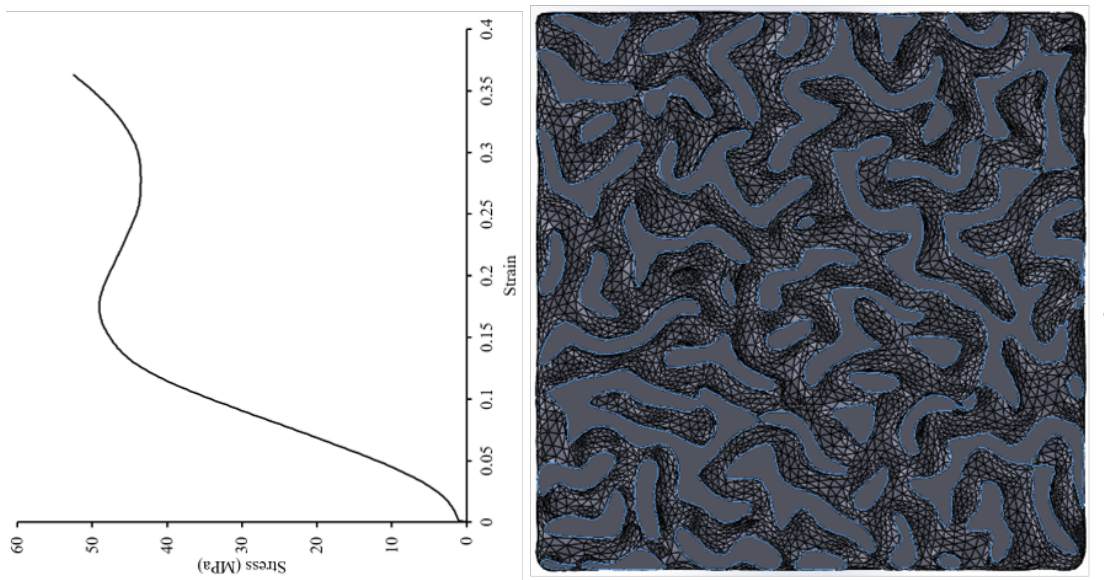

$\stackrel{0}{0}$
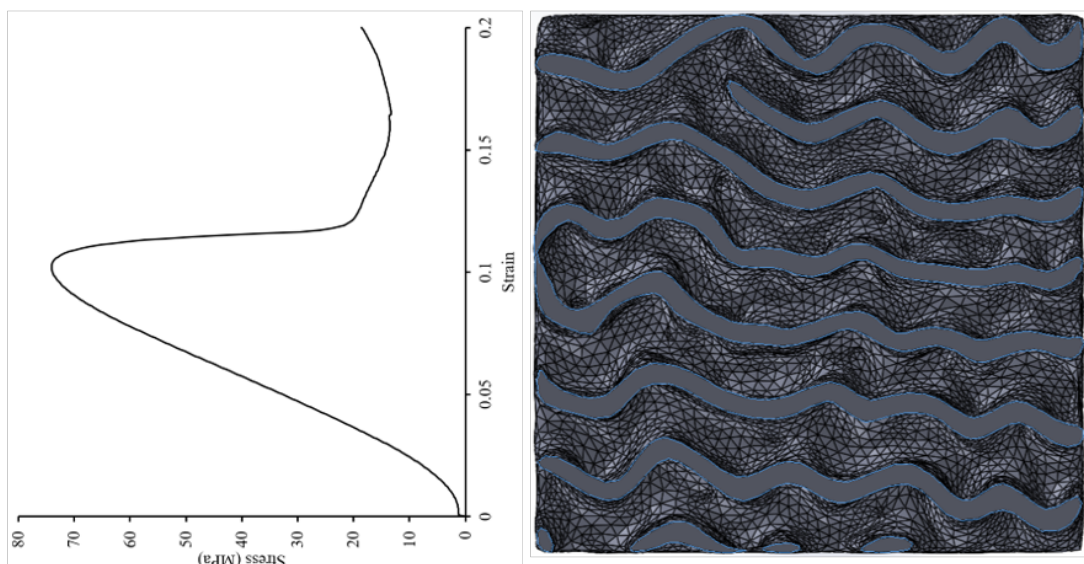

\section{를}
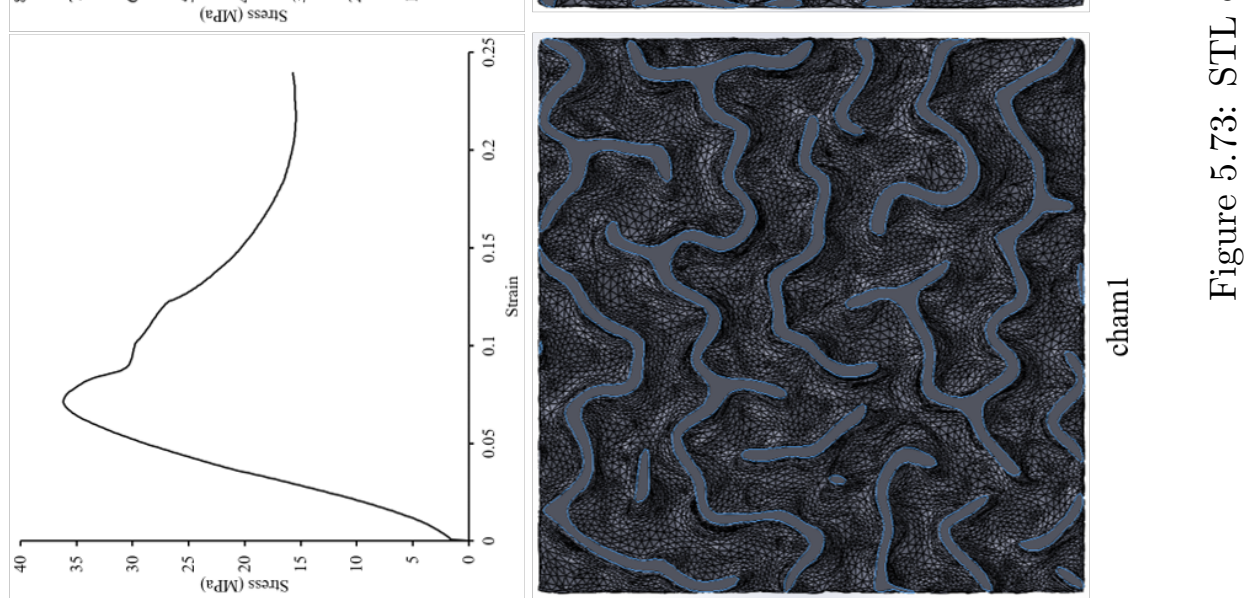

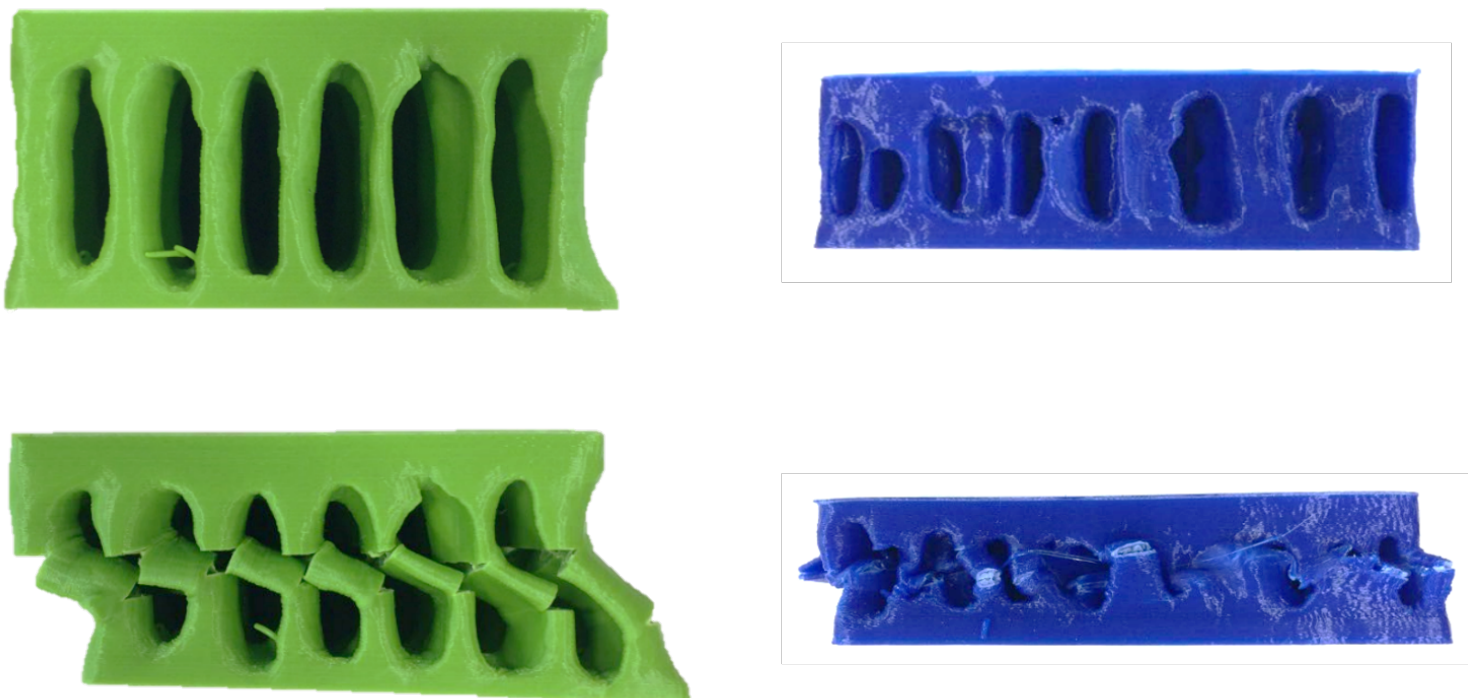

Figure 5.74: Before and after compression photos for cham2 (green) and cham3 (blue).

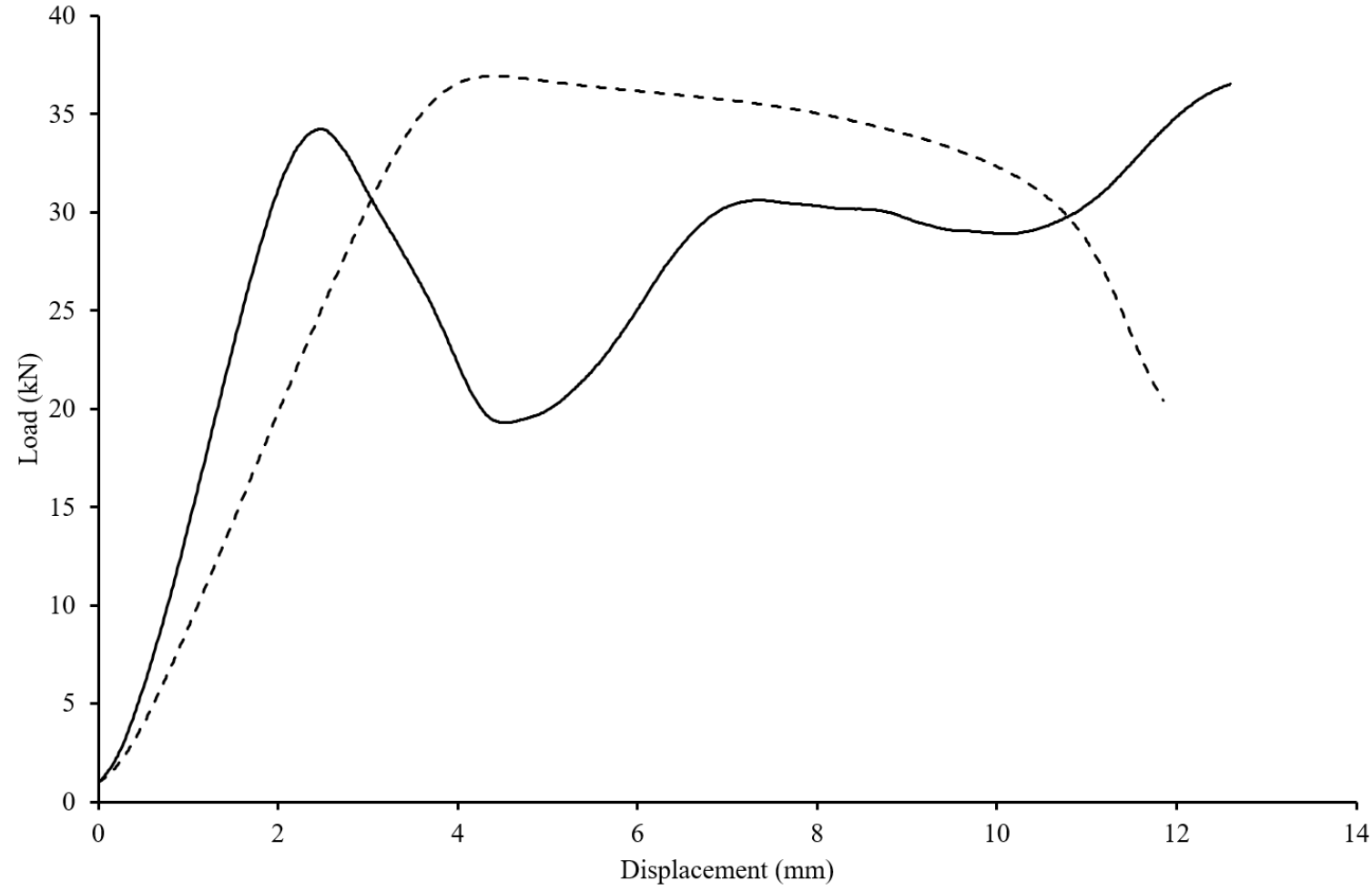

Figure 5.75: Load-displacement curves for three chamber cuttlebone structure (solid line) and three layer honeycomb structure (dashes). 

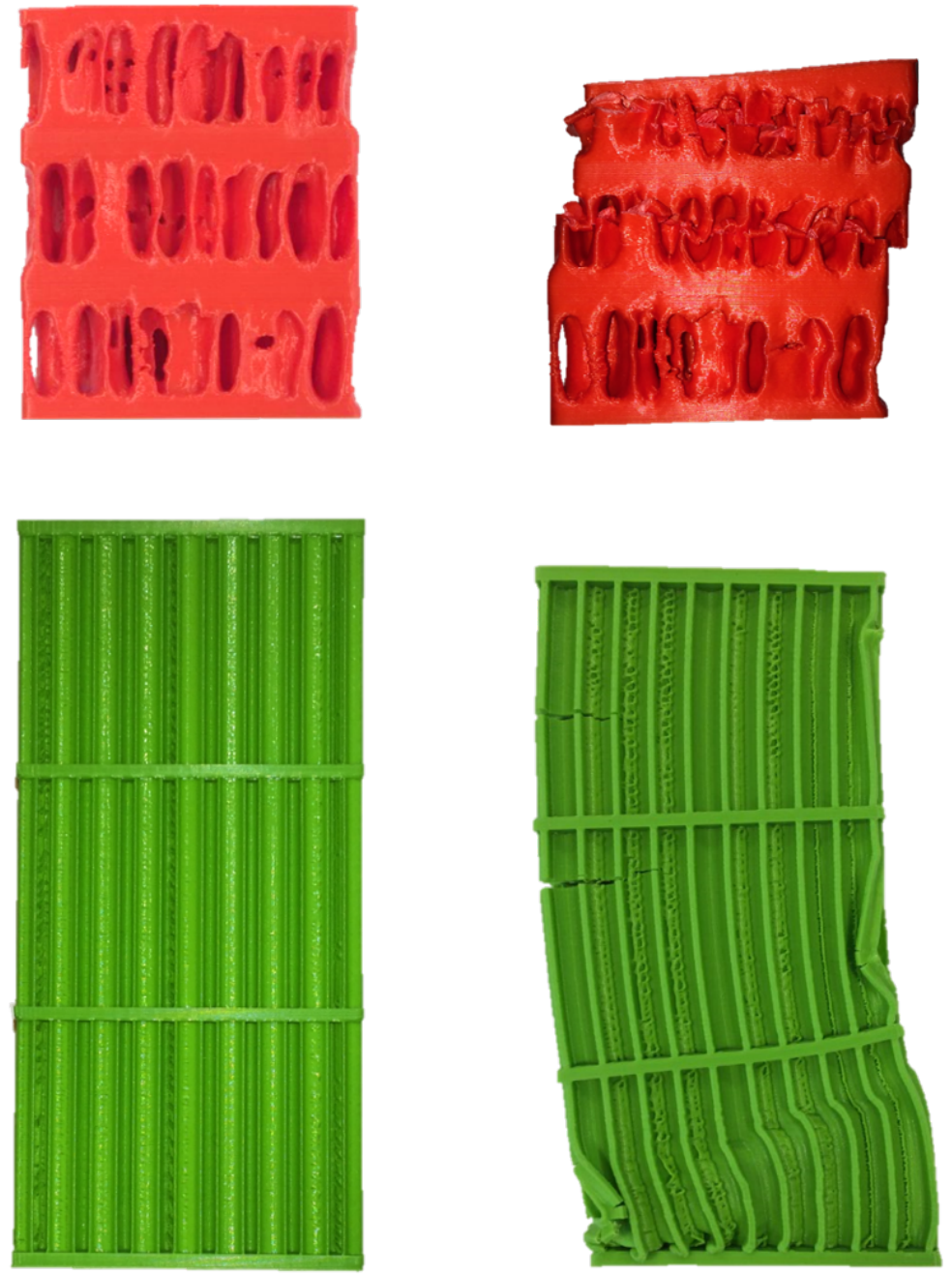

Figure 5.76: Before and after compression photos of three layer cuttlebone structure (red) and three layer honeycomb structure (green). 
material properties and whether they increase the energy absorption potential of cuttlebone structures. The use of a parametric workflow would enable precise evaluation of the various differing characteristics of pillars, for example, the impact of adjusting only pillar thickness, tortuosity or chamber height on the mechanical properties.

Even through using this simplified approach to testing, some hypotheses relating to the mechanical performance of cuttlebone have been supported and strengthened. The results could inform the design of a lightweight, cellular solid for a range of applications.

\subsection{Summary}

The use of X-ray $\mu \mathrm{CT}$ produced a previously unseen amount of detail around the quantification of key chamber and pillar measurements across the cuttlebone in three dimensions. These results identified substantial regional morphological variations, which led to the creation of a suggested framework for pillar count, tortuosity and material-to-air percentage across the entire structure.

An interesting outcome of the morphological investigation was new insight and evidence to support previous findings [225] about the flow of fluid through the cuttlebone when regulating buoyancy. It showed pillar features combining to help control fluid flow in different regions of the cuttlebone. This type of finding is an additional step in the traditional bioinspiration pathway, in that it helps to inform the understanding of form and function in nature.

The 3D data was correlated with high resolution SEM imaging to provide a more complete description across varying length scales. EBSD analysis showed a preferred orientation of aragonite in the $\{100\}$ parallel to the height of the cuttlebone. This knowledge provides significant insight into the growth mechanisms of cuttlebone and this type of information would be of value in the study of biomineralisation.

Chemical analysis highlighted differences in mineralisation across the dorsal shield.

Nanoindentation and hardness tests were performed on polished cross-sections of cuttlebone parallel to the layered structure. Regions of increased organic material showed much lower values of mean hardness and modulus. Compression testing with time-lapse imaging identified a progressive collapse mechanism of the chambers. Individual chambers were then 3D printed and tested to observe the effect of pillar morphology on the resistance to compressive forces. It was found that regions with more tortuosity showed better resistance to compressive stress.

The unique architecture of cuttlebone means it shows great promise for engineering and biomimetic applications. For example, the progressive collapse mechanism could be replicated for energy absorption uses. Also the function of the cuttlebone and its corresponding structure would provide a basis for the design of a sophisticated flotation device. The detailed morphology identified in this work may also provide further insight into areas of the cuttlebone currently used in biomedical applications. 


\section{Chapter 6}

\section{Conclusions}

The goal of this study was to contribute to the ever expanding knowledge of biological materials and systems with the hope of inspiring innovative designs in engineering. The vertebrae of hero shrew (Scutisorex somereni) and cuttlebone of Sepia officinalis were presented as examples of biological materials with enigmatic morphology but little current understanding of their function, therefore presenting huge potential for furthering knowledge of these organisms, and potentially leading to improvements to engineering design.

Hero shrew vertebrae were analysed for the first time using X-ray $\mu \mathrm{CT}$. In addition to capturing information for internal and occluded parts of the vertebrae in $3 \mathrm{D}$, the ability to collect data in this way is a particularly important outcome for structures like these vertebrae due to the rarity of available specimens. The work in this thesis demonstrates this capability for imaging rare or enigmatic specimens non-destructively and permitting significant analyses of morphological features.

Manual segmentation methods were used to separate the vertebral column into individual vertebrae. This process proved to be effective and allowed additive manufacturing to be used to evaluate physical movement between vertebrae, since a hands-on approach was not feasible due to the nature of the specimens. The results, although limited, supported ranges of movement captured by $2 \mathrm{D}$ radiographs from previous research and warrant further work using a more sophisticated approach such as finite element modelling.

The cuttlebone was analysed for the first time in $3 \mathrm{D}$ using a series of X-ray $\mu \mathrm{CT}$ scans. Individual core slices were manually assigned to specific chambers for grouping purposes. The slices were then systematically binarised to capture pillar characteristics for the height of the cuttlebone. This novel approach allowed for quantification of pillars through all the cores which gave a representative overview of pillars in the cuttlebone as a whole.

The wealth of pillar and chamber data collected revealed substantial variation of the internal structure in the cuttlebone. From this, new regional frameworks for several key features of the cuttlebone were suggested. Regions near the siphuncular zone showed high pillar counts, strong pillar alignment and relatively low pillar tortuosity - characteristics well suited to efficient fluid flow. This is in contrast to pillars in regions which are generally 
dry. These pillars were low in count and had high tortuosity, which leads to far less efficient fluid flow. This was evidence to strengthen the understanding of the cuttlebone's ability to regulate buoyancy.

To provide a more complete description of the structure-function relationships, higherresolution structural characterisation, mechanical characterisation and chemical analysis were each performed. High resolution structural characterisation demonstrated clear microstructural differences in the structural components, yet the crystallographic orientation of aragonite was similar across these regions. This knowledge provides vital information into the growth mechanisms of cuttlebone which in turn provides valuable insight in the study of biomineralisation.

Nanoindentation and hardness tests were performed to analyse material properties of the structural components and correlated with chemical analysis which showed that regions of the cuttlebone containing increased organic material had lower values of mean hardness and reduced elastic modulus. Novel time-lapse CT compression testing identified a progressive collapse mechanism of the chambers. To partially support hypotheses made throughout this work linking form and mechanical function, additive manufacturing of geometries obtained from $\mu \mathrm{CT}$ was used as a simple approach to investigate regions of varying pillar morphology and the impact on compressive strength and failure modes.

As a result of this study, further work on the chemical, microstructural and nanomechanical variations seen across the structural components of the cuttlebone has been published [234] and can be found in Appendix B.

A more complete description of these important biological structures is achieved when $\mathrm{X}$-ray $\mu \mathrm{CT}$ is combined with complementary analyses, such as high-resolution electron microscopy, nanoindentation and additive layer manufacturing, which were all used in this study. 


\section{Chapter 7}

\section{Recommendations for Future Work}

Volume data produced using X-ray $\mu \mathrm{CT}$ can be directly used in a range of further applications including finite element modelling and additive layer manufacturing. Material properties acquired through nanoindentation would serve as inputs to the finite element models. The work in this study has provided a strong platform for a number of avenues for further research, some of which are listed below.

\subsection{Hero shrew}

- Finite element analysis would be employed to test current functional hypotheses that the hero shrew uses its spine as leverage to forage for food. Spine mechanics could be tested from CT data produced in this work, or alternatively full body analyses including soft tissue and the muscular system could be developed. This would ultimately need to be validated by live specimens displaying this behaviour. For full body analyses, collaborations with biomechanics specialists would be essential and material properties of the vertebrae and surrounding soft tissue would need to be understood, for example, through nanoindentation. This would also require contrast-enhanced $\mu \mathrm{CT}$ imaging of fully intact hydrated specimens which would need to be fixed and stained with an appropriate contrast agent, in order to visualise the soft tissue in addition to the skeletal structure.

- The full range of movement of the entire vertebrae can be investigated either destructively with a complete specimen or using additive layer manufacturing. An advantage of additive layer manufacturing is that the vertebrae could be modified to investigate direct relationships of tubercle size and position with movement. Simplified models could be used to develop engineering applications in flexible shafts or robotics, for example. 


\subsection{Cuttlebone}

- To further understand fluid flow in different regions of the cuttlebone, finite element modelling coupled with computational fluid dynamics could be employed. The results would help to quantify the impact of cuttlebone geometry on fluid flow. This work would provide a greater understanding of the buoyancy functionality of cuttlebone and could lead to development of flotation devices.

- To further expand on linking mechanical performance to cuttlebone geometry, multimaterial additive layer manufacturing could be used to more accurately represent the changes in material properties found within in pillars and septa. Parametric modelling should also be used to investigate specific functional roles (such as energy absorption through different structural components, and compressive strength) of the pillar morphologies considered in this work. Findings from this could then be used to inform designs of lightweight yet high-strength structures.

- In-situ compression testing would eliminate the challenges encountered in this study and allow the progressive collapse mechanism to be more accurately captured in 3D. This would provide quantitative data which could be taken forward for use with digital volume correlation and therefore the validation of finite element models.

- From a biological perspective, a next step for the developed frameworks could be to consider the ontogeny of the cuttlebone, to increase understanding of the chamber and pillar development though the lifetime of the cuttlefish. 
Appendix A

\section{SolidWorks Sketches}




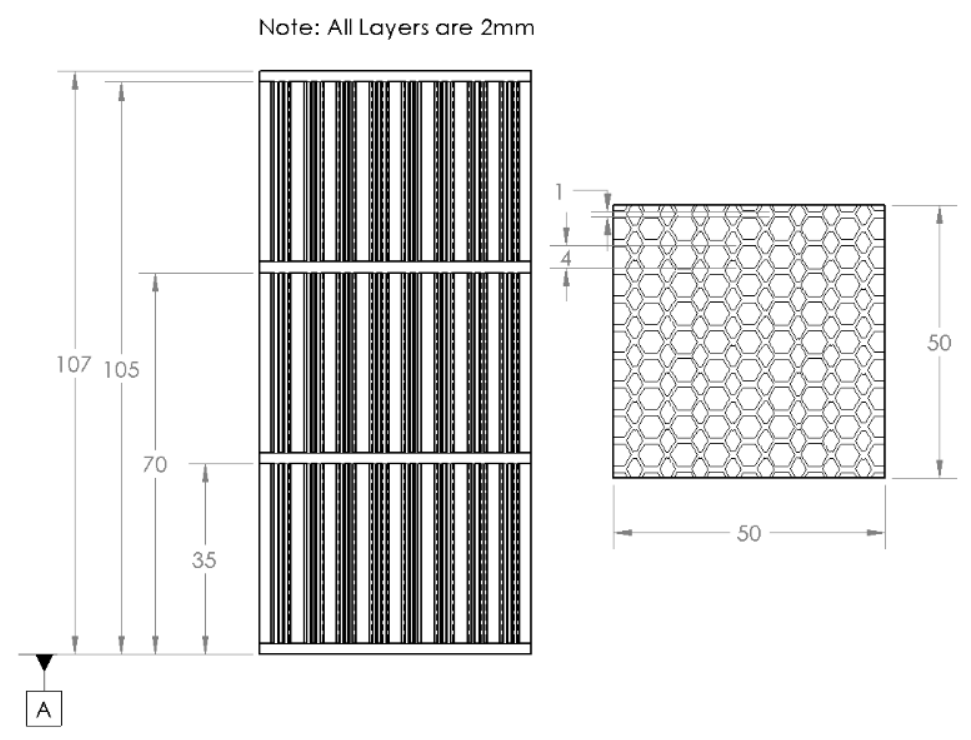

Figure A.1: Sketch of honeycomb structure.

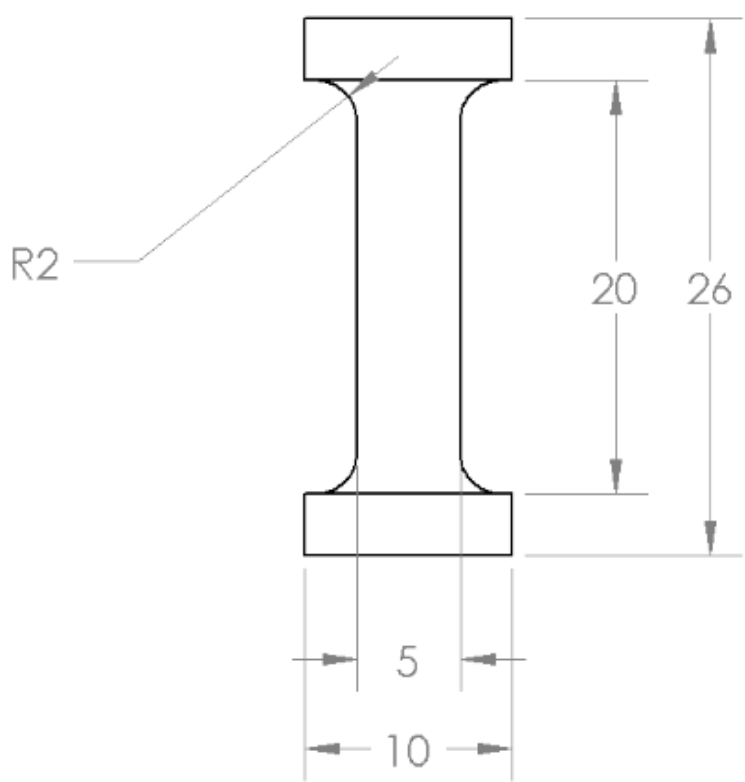

Figure A.2: Sketch of filleted pillar. 
Appendix B

\section{Publications}




\title{
Interrelated chemical-microstructural-nanomechanical variations in the structural units of the cuttlebone of Sepia officinalis
}

\author{
L. North, ${ }^{1}$ D. Labonte, ${ }^{2, a}$ M. L. Oyen, ${ }^{2}$ M. P. Coleman, ${ }^{1}$ H. B. Caliskan, ${ }^{2}$ \\ and R. E. Johnston ${ }^{1, a}$ \\ ${ }^{1}$ Materials Research Centre, College of Engineering, Swansea University, Swansea SA1 8EN, \\ United Kingdom \\ ${ }^{2}$ Cambridge University Engineering Department, Trumpington Street, Cambridge CB2 IPZ, \\ United Kingdom
}

(Received 27 June 2017; accepted 16 October 2017; published online 10 November 2017)

\begin{abstract}
"Cuttlebone," the internalized shell found in all members of the cephalopod family Sepiidae, is a sophisticated buoyancy device combining high porosity with considerable strength. Using a complementary suite of characterization tools, we identified significant structural, chemical, and mechanical variations across the different structural units of the cuttlebone: the dorsal shield consists of two stiff and hard layers with prismatic mineral organization which encapsulate a more ductile and compliant layer with a lamellar structure, enriched with organic matter. A similar organization is found in the chambers, which are separated by septa, and supported by meandering plates ("pillars"). Like the dorsal shield, septa contain two layers with lamellar and prismatic organization, respectively, which differ significantly in their mechanical properties: layers with prismatic organization are a factor of three stiffer and up to a factor of ten harder than those with lamellar organization. The combination of stiff and hard, and compliant and ductile components may serve to reduce the risk of catastrophic failure, and reflect the role of organic matter for the growth process of the cuttlebone. Mechanically "weaker" units may function as sacrificial structures, ensuring a stepwise failure of the individual chambers in cases of overloading, allowing the animals to retain near-neutral buoyancy even with partially damaged cuttlebones. Our findings have implications for our understanding of the structure-property-function relationship of cuttlebone, and may help to identify novel bioinspired design strategies for lightweight yet high-strength foams. (C) 2017 Author(s). All article content, except where otherwise noted, is licensed under a Creative Commons Attribution (CC BY) license (http://creativecommons.org/licenses/by/4.0/). https://doi.org/10.1063/1.4993202
\end{abstract}

Cuttlefish maintain near-neutral buoyancy at varying diving depths through the use of a specialized floatation device, frequently referred to as cuttlebone, which needs to combine high strength with minimum weight. Despite a porosity exceeding $90 \%,{ }^{1}$ the cuttlebones of some species withstand pressures encountered at up to $500 \mathrm{~m}$ in diving depth. ${ }^{2-4}$ This exceptional combination of high compressive strength, porosity, and permeability is extremely desirable for biomimetic and biomedical structural materials, including templates for tissue scaffolds,,${ }^{5,6}$ hydroxyapatite scaffolds, ${ }^{7-9}$ and bone cements. $^{10}$

The remarkable performance of the cuttlebone is linked to its structural architecture: cuttlebone is composed of calcium carbonate $\left(\mathrm{CaCO}_{3}\right)$ in its aragonite polymorph with a mixture of $\beta$-chitin and other protein complexes, and comprises two main structural units: a dorsal shield and a series of chambers. The continuous chambers are separated by parallel septa and supported by a complex arrangement of meandering plates [from here on referred to as "pillars," see Figs. 1(a)-1(d)].

${ }^{\mathrm{a}}$ Authors to whom correspondence should be addressed: r.johnston@swansea.ac.uk and dl416@cam.ac.uk 

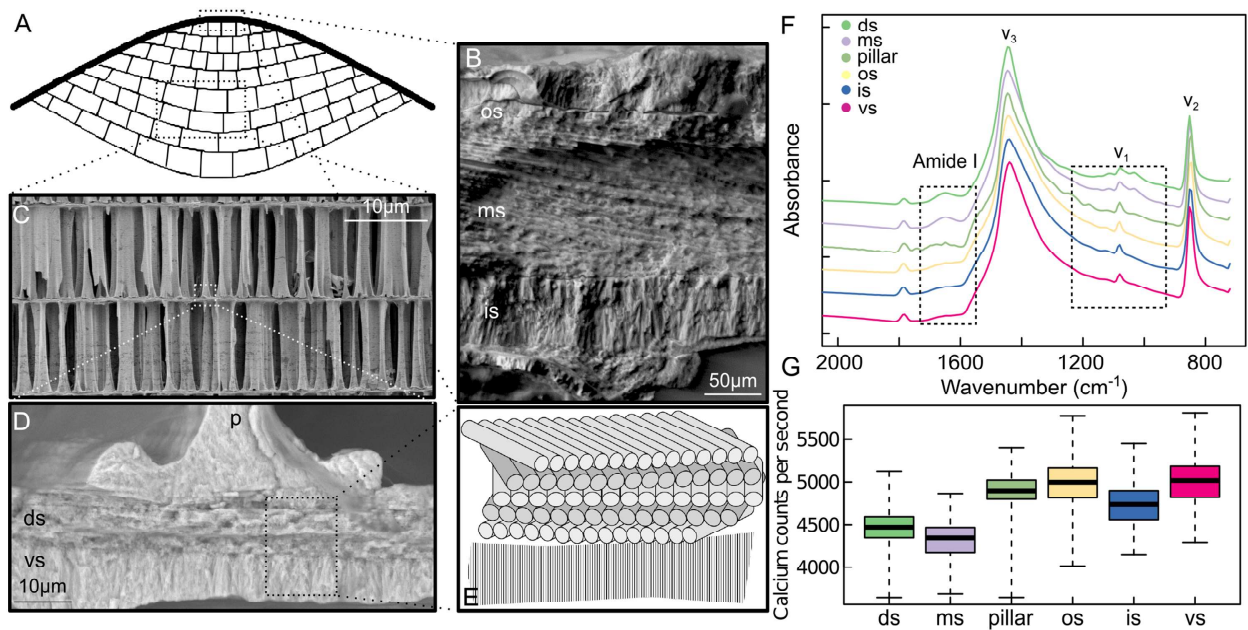

FIG. 1. (a) Schematic cross section of the cuttlebone, indicating the dorsal shield (thick black line) and the chambers (cellular network, thin black lines), which consists of septa and pillars. Scanning electron micrographs of (b) the three distinct layers in the dorsal shield, (c) pillars supporting adjacent chambers, (d) the intersection of a septum and pillar highlighting the different microstructures within a septum. (e) Schematic of the change in direction of fibers in the septum. (f) Chemical analyses of the distinct layered structures in cuttlebone in the form of attenuated total internal reflection (ATR) Fourier Transform Infrared (FTIR) spectra. Each spectrum was normalized and then shifted vertically for clarity. Dashed boxes highlight the aragonite "fingerprint" peak at $1080 \mathrm{~cm}^{-1}$ and the presence of organic macromolecules at around $1650 \mathrm{~cm}^{-1}$, respectively. (g) Box-whisker plots illustrating the variation in calcium content between the three layers within the dorsal shield and the two layers within septa, as measured with energy dispersive X-ray spectroscopy (EDS). FTIR and EDS reveal clear differences in the amount of organic matter and the calcium content between the layers of both the dorsal shield and septa, which are similar among prismatic and lamellar crystal organizations, respectively. p—pillar, os—outer shield, ms—-middle shield, is—inner shield, ds—dorsal septum, and vs—ventral septum.

The macro-structure, ${ }^{1,11-14}$ growth mechanisms, ${ }^{11,15}$ bulk mechanical performance,,${ }^{1,4,12,16}$ and chemical composition of cuttlebone, ${ }^{17}$ as well as the role of the organic constituents in the formation of the chambers and the organization of the crystals have been studied in some detail. $1,11,15,17,18$ However, the structure-property relationships across the identified structural units of this complex biomaterial have not yet been fully investigated (but see Refs. 1,4,16,19-21), although these units were first identified more than 180 years ago. ${ }^{22}$ In this work, we aim to close this gap and provide a detailed chemical, structural, and mechanical analysis of the different structural units, using scanning electron microscopy (SEM), energy dispersive X-ray spectroscopy (EDS), Fourier transform infrared spectroscopy (FTIR), and nanoindentation.

Fresh specimens of Sepia officinalis Linnaeus, 1758, were acquired commercially from around the UK coastline. The cuttlebone was dissected, sectioned into smaller samples (approximately $2 \mathrm{~cm}^{3}$ ) taken from the central region of the cuttlebone, and embedded in epoxy resin (EpoFix, Struers) using vacuum impregnation. Embedded specimens were diamond and colloidal silica polished to $0.04 \mu \mathrm{m}$ finish, and a $7 \mathrm{~nm}$ carbon coating was applied using a Quorum Technologies Q150T turbo-pumped carbon coater.

EDS analysis in the form of line scans at $10 \mathrm{kV}$ was carried out on transverse sections to provide information on the calcium content of both the dorsal shield and the chambers, using a Carl Zeiss Crossbeam 540 FEG SEM equipped with an SMax50 EDS detector, controlled via the Aztec software (Oxford Instruments). 10 line scans were performed with sampling separations within each line of $3.3 \mu \mathrm{m}$ and $0.14 \mu \mathrm{m}$ for the dorsal shield and the septa, and 6 line scans with sampling separations of $2.4 \mu \mathrm{m}$ for the pillars, respectively; each line was measured three times and the arithmetic mean was used in further analyses.

Spectroscopic analysis of the samples was conducted using a PerkinElmer Spotlight400 FTIR fitted with an attenuated total internal reflection (ATR) imaging mode microscope (PerkinElmer, Waltham, Massachusetts, USA). The wavelength resolution was set to $12 \mathrm{~cm}^{-1}$, and eight scans per pixel were collected, with a pixel size of $1.56 \mu \mathrm{m}$. 
Mechanical properties of the different structural units of the cuttlebone were measured in ambient conditions with a TI 950 Triboindenter (Hysitron, Eden Prairie, USA), equipped with a diamond Berkovich indenter with a tip radius of $150 \mathrm{~nm}$. Indentations were conducted in closed-loop displacement-control with a 5-10-5 s load-hold-unload trapezoidal load profile, and a peak displacement of $200 \mathrm{~nm}$ unless stated otherwise. Indentation hardness $(H)$ and indentation modulus $\left(E^{\prime}\right)$ were extracted from the force-displacement data using the Oliver and Pharr method ${ }^{23}$ In order to quantify the variation in $H$ and $E^{\prime}$ across the shield, 18 horizontal (orthogonal to the dorso-ventral axis) lines of 25 vertical (along the dorso-ventral axis) indents were placed across the shield of one specimen, starting on the septum adjacent to the ventral layer of the shield, and finishing on the surrounding epoxy. The vertical and horizontal spacings were $20 \mu \mathrm{m}$ and at least $20 \mu \mathrm{m}$, respectively. The results were averaged either according to their vertical position for plotting, or according to "region" for further statistical analyses.

The mechanical properties of 14 randomly selected pillars were measured at various points along their length. The exact position varied across the pillars due to variable cross-sectional width, and we avoided regions that were thin or close to air gaps; the minimum vertical spacing was $20 \mu \mathrm{m}$. A linear regression of $H$ and $E^{\prime}$ against the indentation position normalized by pillar length revealed that neither differed significantly along the length of the pillars ( $t$-test for the slope, $t$-statistic $=0.40$, $\mathrm{p}$-value $=0.69$ and $\mathrm{t}$-statistic $=0.61$, $\mathrm{p}$-value $=0.52$ for $\mathrm{E}^{\prime}$ and $\mathrm{H}$, respectively). In order to capture the variation of material properties across pillars, 35 further pillars were indented, with three indents per pillar, located approximately at the bottom, middle, and top relative to the pillar length. All results were pooled for further analyses.

Preliminary microscopy of the septum suggested that it contained at least two distinct regions [see Figs. 1(d)], separated roughly at the midline [see also Refs. 15 and 18]. In order to quantify the properties of these two regions, 60 indents were placed approximately centrally in each region, with a horizontal spacing of at least $20 \mu \mathrm{m}$. In addition, we used the accelerated property-mapping (XPM) feature of the Triboindenter to visualize localized changes in material properties. XPM measurements were conducted in force-control, as recommended by the manufacturer. Mechanical properties of the shield were mapped with a $3 \times 148(3 \mu \mathrm{m}$ vertical $\times 3 \mu \mathrm{m}$ horizontal spacing $)$ grid, a peak load of $1 \mathrm{mN}$, and a 1-2-1 s trapezoidal load profile. The variation in mechanical properties at the transition between pillar and septum was visualized with a $12 \times 25(5 \mu \mathrm{m}$ horizontal $\times 2 \mu \mathrm{m}$ vertical $)$ grid, with a peak load of $0.8 \mathrm{mN}$, and a 1-5-1 s trapezoidal loading profile. Only measurements with a contact depth exceeding $60 \mathrm{~nm}$ were used for further analyses, and data excluded by this criterion were linearly interpolated from the surrounding data points. All XPM data were used for plotting mechanical property maps and were not included in the quantitative statistical analysis.

The main structural components of the cuttlebone-the dorsal shield, the septa, and the pillarscan be readily identified by light and electron microscopy (Fig. 1). The dorsal shield consists of two layers formed of prismatic tubercles that encapsulate a middle layer with lamellar aragonite structure [Fig. 1(b) and Ref. 13]. This difference is also apparent in backscattered electron imaging, where the sandwiched layer shows a reduced atomic density [see Fig. S1(a) of the supplementary material]. A similar structural arrangement was found for the septa, which are split at about midline [Figs. 1(d) and 1(e)], confirming previous work, which has shown that the dorsal part of the septa has an aragonite lamella-fibrillar microstructure with fibers oriented in multiple directions on different planes. ${ }^{15,24}$ The prismatic structure of the ventral part, in turn, consists of aragonite fibers oriented parallel to the pillar height, which are crystallographically continuous into the adjacent pillar [Figs. 1(d) and 1(e)].

These structural differences were also evident in both FTIR spectra and EDS data. While the "fingerprint" peaks of aragonite were present in all spectra, components with a lamellar ultrastructure showed a distinct broadening of the v1-mode peak $\left(1080 \mathrm{~cm}^{-1}\right)$, and additional low-intensity peaks between 1000 and $1300 \mathrm{~cm}^{-1}$ as well as at $1650 \mathrm{~cm}^{-1}$, indicative of the presence of a significant amount of organic macromolecules [Fig. 1(f)] ${ }^{18}$ Components with a prismatic morphology, in turn, had a larger Ca count per second (cps), as measured via EDS [4915 \pm 16.1 compared to $4392 \pm 10.75$ $\mathrm{cps}$, for detailed data on individual layers, see Fig. 1(g) and Table SI of the supplementary material]. This difference translated into calcium weight percentages of $46.5 \%$ for the prismatic and $45.15 \%$ for the lamellar components. 
While these differences may sound minor, in combination they lead to a marked difference in mechanical properties. Nanoindentation revealed that units with prismatic structure have an indentation modulus which exceeds that of lamellar components by a factor between two and three, and an indentation hardness which is up to a factor of ten larger (see Figs. 2 and 3 and Table SI of the supplementary material for detailed data). For both the dorsal shield and the septa, the changes in material properties between prismatic and lamellar building blocks appear to be abrupt and occurred on a microscopic length scale, as evident from the XPM [see Figs. 2(c) and 3, respectively]. Note that the ventral septa and the outer shield have a similar chemical composition but vary in indentation modulus and hardness, likely due to edge effects arising from the narrow width of the ventral septum $(<10 \mu \mathrm{m})$.

Cuttlebone needs to be as light as possible to maximize buoyancy but as strong as necessary to ensure sufficient safety factors. These conflicting demands are reflected in a complex bio-architecture, featuring at least six different structural units, which differ not only in their microstructure and chemical composition, but also in their mechanical properties (see Figs. 1-3). Stiff and hard layers with prismatic crystal organization alternate with more compliant and ductile zones, organized in a lamellar form and enriched with organic macromolecules. The inclusion of "weaker" layers may at first seem surprising, as the maximum diving depth is directly related to the mechanical properties for

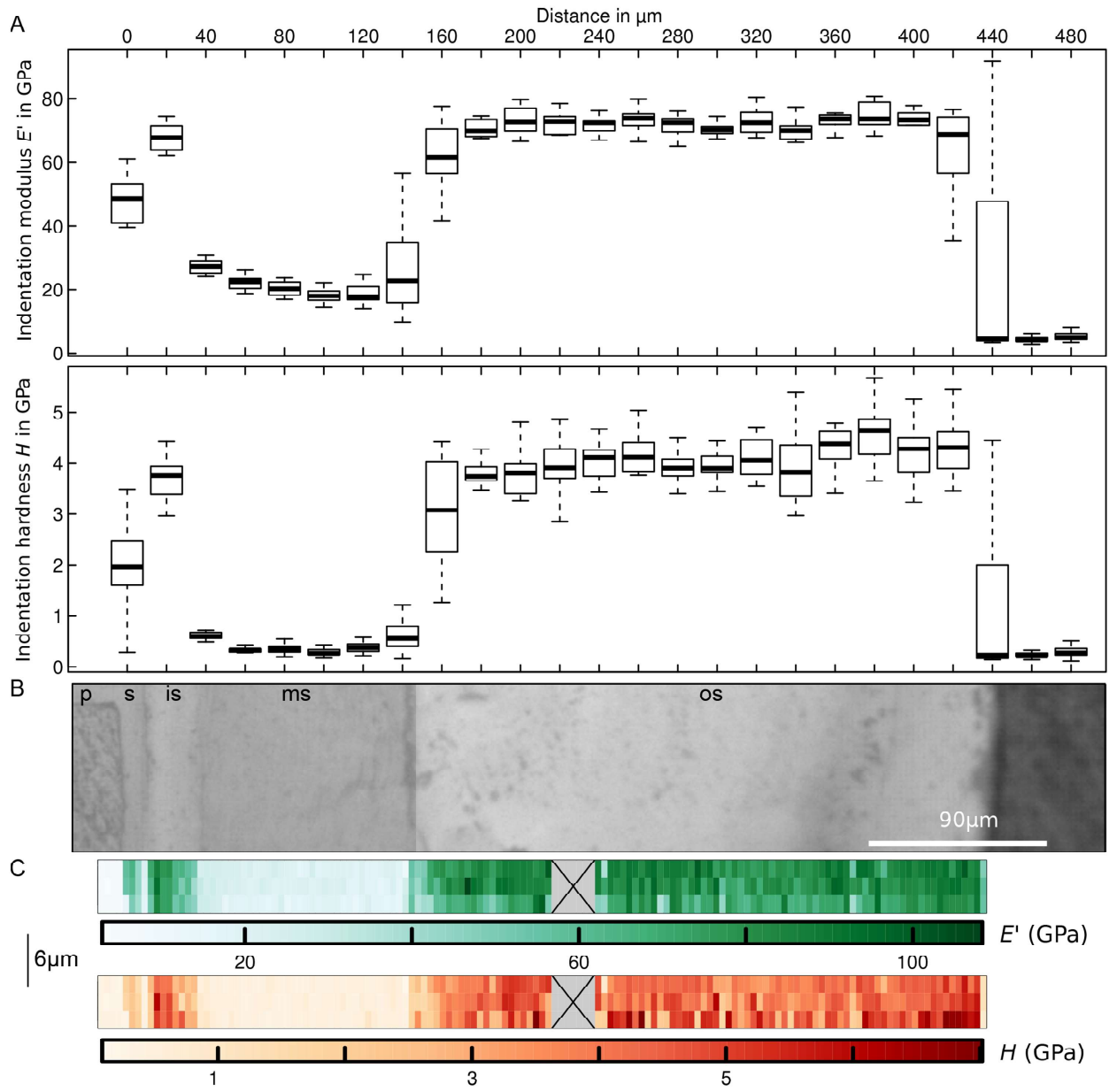

FIG. 2. (a) Box-whisker plots illustrating the variation in indentation modulus and hardness across the different layers in the dorsal shield, which are shown in the light microscopy image in (b) ( $\mathrm{n}=18$ per box-whisker plot). The clear differences in indentation hardness and modulus and the sharp transition between the different layers are visualized further in (c), which shows data collected with the accelerated property mapping feature of the 950 Triboindenter. The crossed-out, grey block in (c) is an unmapped region. p-pillar, os-outer shield, ms - middle shield, is-inner shield, s-septum. 

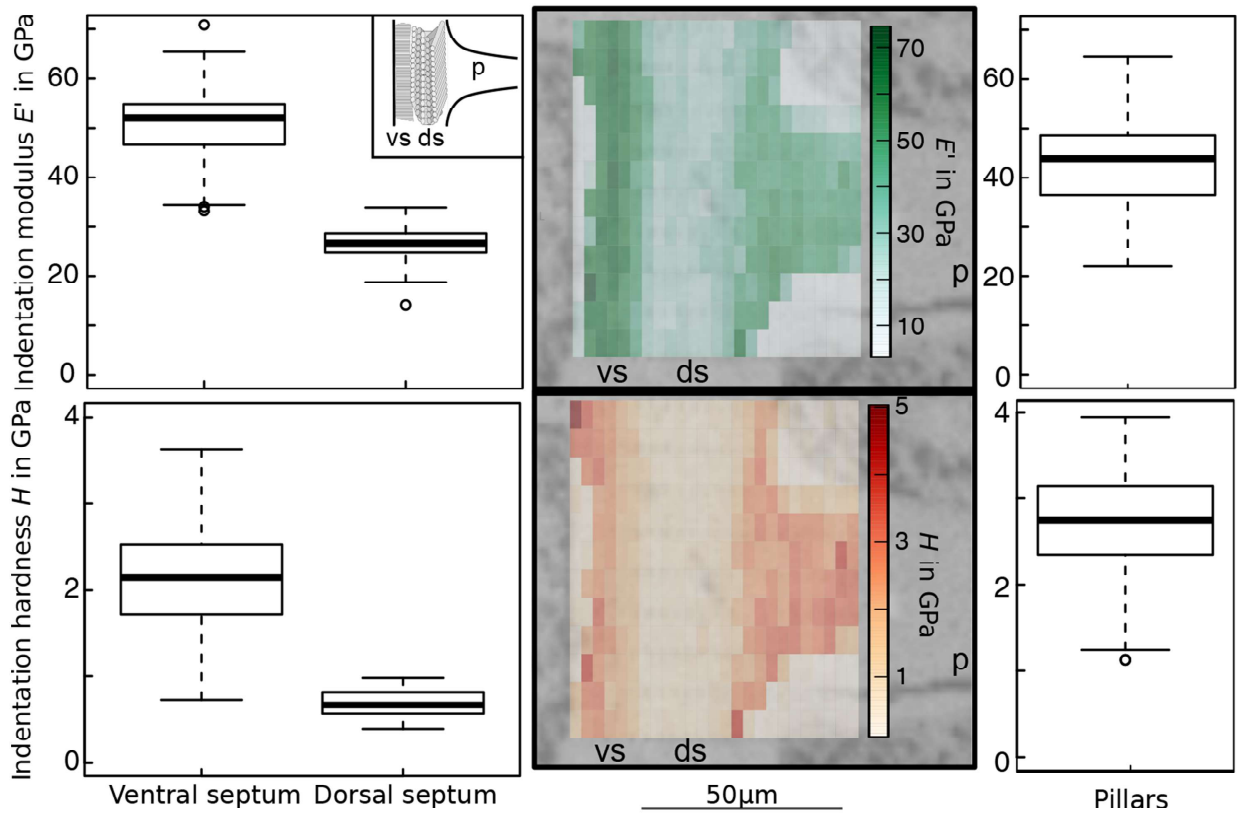

FIG. 3. Box-whisker plots illustrating the variation in indentation modulus and hardness in the two distinct layers in the septa (left-hand side, $n=60$ per layer) and the pillars (right-hand side, $n=275$ for 49 different pillars). The clear differences in indentation hardness and modulus and the apparent sharp transition between the different layers are visualized further in the central region of the figure, which shows data collected with the accelerated property mapping feature of the 950 Triboindenter, overlaid on an light microscopy image of the corresponding region. p — pillar, ds — dorsal septum, vs—ventral septum.

a given geometric arrangement. ${ }^{4,12}$ However, several arguments may be brought forward to rationalize this design strategy.

First, variations in indentation modulus between adjacent layers may represent a toughening mechanism. Cracks are effectively arrested at the interface between adjacent layers if the ratio of their elastic moduli is greater than about $5,{ }^{25}$ as, for example, observed in hexactinellid sponges, ${ }^{26}$ albeit at a much smaller scale. Similar mechanisms have also been described for nacre, ${ }^{25,27,28}$ again at a much smaller scale than described here. Both the middle layer of the dorsal shield and the dorsal septum might be particularly effective in arresting cracks, as they have an unusually small ratio between indentation hardness and modulus (about half of 0.05, the value typical for biological materials), which is a proxy for the tendency of a material to undergo elastic versus inelastic deformation. ${ }^{29}$ Hence, these layers may absorb energy by inelastic deformation instead of feeding it into the propagation of cracks. Indeed, X-ray microCT investigation of the dorsal shield revealed several cases where cracks seemingly originating in the outer shield failed to penetrate the central layer of the shield, and instead were arrested at the interface, providing support for our hypothesis [see Fig. S1(c) of the supplementary material].

Second, and related to the first point, the inclusion of "sacrificial" layers may represent a strategy to ensure controlled and localized structural failure in case of overloading, for example, by directing gross failure to the pillars. The question remains, however, which failure mechanism would be most beneficial for the organism. Overloaded pillars collapse, resulting in a stepwise failure and densification of individual chambers [see Fig. S1(b) of the supplementary material for a 4D X-ray microtomography visualization]. Cuttlefish can survive multiple of such chamber failures, ${ }^{3}$ and the variation in mechanical properties identified in this study might be key to avoid a damaging through-thickness crack in the dorsal shield, which would compromise near-neutral buoyancy.

Third, the proteinous organic matrix plays a major role in cuttlebone formation, ${ }^{15,18,30}$ so that variations in the amount of organic molecules might simply reflect a developmental pattern rather than a mechanical adaptation. 
The way in which the cuttlebone fails when overloaded also has implications for the link between cuttlebone morphology and maximum diving depth established by earlier work. ${ }^{2-4}$ Previous studies have used beam or plate theory to study the mechanical performance of the Sepia cuttlebone with reasonable success. ${ }^{4,12}$ While these studies assumed that failure occurs via elastic buckling of the pillars, there are two other competitive failure modes in foam-like solids, "plastic" collapse, or brittle crushing. ${ }^{31}$ Notably, the maximum sustainable stress, $\sigma$, predicted for the two latter failure modes is directly proportional to the aspect ratio characterizing the repetitive unit, which we define here as wall thickness, $t$, versus wall length, $l$. In contrast, $\sigma \sim(\mathrm{t} /)^{2}$ for elastic buckling ${ }^{31}$ implying that elastic buckling will be the dominant failure mode for structures with small aspect ratios (see supplementary material). However, for animals which dive to deeper depths-and hence typically possess chambers with larger aspect ratios ${ }^{4}$ - the mechanical performance limit might be set by plastic collapse or brittle crushing instead, and indeed the "frayed" failure of some cuttlebone under compression suggests that brittle crushing may be dominant. ${ }^{1,16}$

The identified variations in ultrastructure, chemistry, and mechanical properties across the structural units of the cuttlebone are not only relevant for our understanding of the structure-function relationship in the cuttlebone, but also for the design of bioinspired foams which combine low weight with high strength.

See supplementary material for a summary of the mechanical property data, further electron microscopy, X-ray microCT of layer failure, and a description of possible cuttlebone failure based on a foam model.

The work was supported by the Advanced Imaging of Materials (AIM) facility (EPSRC Grant No. EP/M028267/1), the European Social Fund (ESF) through the European Union's Convergence programme administered by the Welsh Government, the United States Army Corps of Engineers, Engineer Research and Development Centre (ERDC), the Denman Baynes Senior Research Fellowship (to D.L.), the Islamic Development Bank \& the Cambridge Commonwealth, European and International Trust PhD Scholarship (to H.B.C.), and an EPSRC Doctoral Training Award (EPSRC Grant No. EP/K502935/1 to L.N.). The authors would like to thank Peter Davies for microscopy technical assistance and Dr. Ed Pope for discussions on Sepia officinalis behavior. All data created during this research are openly available from the Swansea University data archive at https://doi.org/10.5281/zenodo.1039667.

${ }^{1}$ J. D. Birchall and N. L. Thomas, "On the architecture and function of cuttlefish bone," J. Mater. Sci. 18(7), 2081-2086 (1983).

${ }^{2}$ P. D. Ward and S. V. Boletzky, "Shell implosion depth and implosion morphologies in three species of Sepia (Cephalopoda) from the Mediterranean Sea," J. Mar. Biol. Assoc. U. K. 64(4), 955 (1984).

${ }^{3}$ P. Neige and S. V. Boletzky, "Morphometrics of the shell of three Sepia species (Mollusca: Cephalopoda): Intra-and interspecific variation," Zool. Beitr. 38, 137-156 (1997).

${ }^{4}$ K. Sherrard, "Cuttlebone morphology limits habitat depth in eleven species of Sepia (Cephalopoda: Sepiidae)," Biol. Bull 198(3), 404-414 (2000)

${ }^{5}$ J. H. G. Rocha, A. F. Lemos, S. Agathopoulos, P. Valerio, S. Kannan, F. N. Oktar et al., "Scaffolds for bone restoration from cuttlefish," Bone 37(6), 850-857 (2005).

${ }^{6}$ W. Ogasawara, W. Shenton, S. A. Davis, and S. Mann, "Template mineralization of ordered macroporous chitin-silica composites using a cuttlebone-derived organic matrix," Chem. Mater. 12(10), 2835-2837 (2000)

${ }^{7}$ H. Ivankovic, G. G. Ferrer, E. Tkalcec, S. Orlic, and M. Ivankovic, "Preparation of highly porous hydroxyapatite from cuttlefish bone," J. Mater. Sci.: Mater. Med. 20(5), 1039-1046 (2009).

${ }^{8}$ D. Milovac, G. Gallego Ferrer, M. Ivankovic, and H. Ivankovic, "PCL-coated hydroxyapatite scaffold derived from cuttlefish bone: Morphology, mechanical properties and bioactivity,” Mater. Sci. Eng., C 34, 437-445 (2014).

${ }^{9}$ E. Battistella, S. Mele, I. Foltran, I. G. Lesci, N. Roveri, P. Sabatino et al., "Cuttlefish bone scaffold for tissue engineering: A novel hydrothermal transformation, chemical-physical, and biological characterization," J. Appl. Biomater. Funct. Mater. 2(10), 99-106 (2012).

${ }^{10}$ S. García-Enriquez, H. E. R. Guadarrama, I. Reyes-González, E. Mendizábal, C. F. Jasso-Gastinel, B. García-Enriquez et al., "Mechanical performance and in vivo tests of an acrylic bone cement filled with bioactive Sepia officinalis cuttlebone," J. Biomater. Sci., Polym. Ed. 21(1), 113-125 (2010).

${ }^{11}$ K. Tanabe, Y. Fukuda, and Y. Ohtsuka, "New chamber formation in the cuttlefish Sepia esculenta Hoyle," Venus 44, 55-67 (1985).

${ }^{12}$ D. Gower and J. F. V. Vincent, "The mechanical design of the cuttlebone and its bathymetric implications," Biomimetics 4, $37-58(1996)$

${ }^{13}$ J. J. A. Appellöf, Die Schalen Von Sepia, Spirula Und Nautilus: Studien Über Den Bau Und Das Wachsthum (PA Norsted \& Söner, 1893), Vol. 25 
${ }^{14} \mathrm{~K}$. Bandel and S. V. Boletzky, "Comparative study of the structure, development and morphological relationships of chambered cephalopod shells," Veliger 21(3), 313 (1979).

${ }^{15}$ A. G. Checa, J. H. E. Cartwright, I. Sánchez-Almazo, J. P. Andrade, and F. Ruiz-Raya, "The cuttlefish Sepia officinalis (Sepiidae, Cephalopoda) constructs cuttlebone from a liquid-crystal precursor," Sci. Rep. 5(1), 11513 (2015).

${ }^{16}$ S. A. Tekalur, M. Raetz, and A. Dutta, "Composite design through biomimetic inspirations," in Experimental and Applied Mechanics, edited by T. Proulx (Springer, New York, NY, 2011), Vol. 6, pp. 355-357.

${ }^{17}$ M. Florek, E. Fornal, P. Gómez-Romero, E. Zieba, W. Paszkowicz, J. Lekki et al., "Complementary microstructural and chemical analyses of Sepia officinalis endoskeleton,” Mater. Sci. Eng., C 29(4), 1220-1226 (2009)

${ }^{18}$ C. Le Pabic, A. Marie, B. Marie, A. Percot, L. Bonnaud-Ponticelli, P. J. Lopez et al., "First proteomic analyses of the dorsal and ventral parts of the Sepia officinalis cuttlebone," J. Proteomics 150, 63-73 (2017)

${ }^{19}$ J. Cadman, S. Zhou, Y. Chen, W. Li, R. Appleyard, and Q. Li, "Characterization of cuttlebone for a biomimetic design of cellular structures," Acta Mech. Sin. 26(1), 27-35 (2010)

${ }^{20}$ J. Cadman, C.-C. Chang, J. Chen, Y. Chen, S. Zhou, W. Li et al., "Bioinspired lightweight cellular materials-Understanding effects of natural variation on mechanical properties," Mater. Sci. Eng., C 33(6), 3146-3152 (2013).

${ }^{21}$ A. Knöller, T. Runčevski, R. E. Dinnebier, J. Bill, and Z. Burghard, "Cuttlebone-like $\mathrm{V}_{2} \mathrm{O}_{5}$ nanofibre scaffolds-Advances in structuring cellular solids," Sci. Rep. 7, 42951 (2017).

${ }^{22}$ E. Griffith and E. Pidgeon, "The Mollusca and Radiata," in Arranged by the Baron Cuvier, with Supplementary Additions to Each Order (Whittaker and Company, London, 1834)

${ }^{23}$ W. C. Oliver and G. M. Pharr, "An improved technique for determining hardness and elastic modulus using load and displacement sensing indentation experiments," J. Mater. Res. 7(6), 1564-1583 (1992).

${ }^{24}$ L. A. Doguzhaeva and E. Dunca, "Siphonal zone structure in the cuttlebone of Sepia officinalis," Swiss J. Palaeontol. 134(2), 167-176 (2015)

${ }^{25}$ P. Fratzl, H. S. Gupta, F. D. Fischer, and O. Kolednik, "Hindered crack propagation in materials with periodically varying Young's modulus-Lessons from biological materials," Adv. Mater. 19(18), 2657-2661 (2007).

${ }^{26}$ J. Aizenberg, J. C. Weaver, M. S. Thanawala, V. C. Sundar, D. E. Morse, and P. Fratzl, "Skeleton of Euplectella sp. Structural hierarchy from the nanoscale to the macroscale," Science 309(5732), 275-278 (2005)

${ }^{27} \mathrm{H}$. Kakisawa and T. Sumitomo, "The toughening mechanism of nacre and structural materials inspired by nacre," Sci. Technol. Adv. Mater. 12(6), 64710 (2011)

${ }^{28}$ O. Kolednik, J. Predan, F. D. Fischer, and P. Fratzl, "Bioinspired design criteria for damage-resistant materials with periodically varying microstructure," Adv. Funct. Mater. 21(19), 3634-3641 (2011).

${ }^{29}$ D. Labonte, A.-K. Lenz, and M. L. Oyen, "On the relationship between indentation hardness and modulus, and the damage resistance of biological materials," Acta Biomater. 57, 373-383 (2017).

${ }^{30}$ V. Čadež, S. D. Škapin, A. Leonardi, I. Križaj, S. Kazazić, B. Salopek-Sondi et al., "Formation and morphogenesis of a cuttlebone's aragonite biomineral structures for the common cuttlefish (Sepia officinalis) on the nanoscale: Revisited," J. Colloid Interface Sci. 508, 95-104 (2017)

${ }^{31}$ L. J. Gibson and M. F. Ashby, Cellular Solids: Structure and Properties, Cambridge Solid State Science Series, 2nd ed. (Cambridge University Press, Cambridge, 2001), p. 510, 1. Paperback ed. (with corr.), transferred to digital printing. 


\section{Bibliography}

[1] J. Vincent, Structural Biomaterials. Princeton University Press, 1991.

[2] A. Srinivasan, G. Haritos, and F. Hedberg, "Biomimetics: Advancing man-made materials through guidance from nature," Applied Mechanics Reviews, vol. 44, no. 11, pp. 463-482, 1991.

[3] J. Currey, A. Nash, and W. Bonfield, "Calcified cuticle in the stomatopod smashing limb," Journal of Materials Science, vol. 17, no. 7, pp. 1939-1944, 1982.

[4] J. C. Weaver, G. W. Milliron, A. Miserez, K. Evans-Lutterodt, S. Herrera, I. Gallana, W. J. Mershon, B. Swanson, P. Zavattieri, E. DiMasi, et al., "The stomatopod dactyl club: a formidable damage-tolerant biological hammer," Science, vol. 336, no. 6086, pp. 1275-1280, 2012.

[5] C. Levi, J. Barton, C. Guillemet, E. Bras, and P. Lehuede, "A remarkably strong natural glassy rod: the anchoring spicule of themonorhaphis sponge," Journal of materials science letters, vol. 8, no. 3, pp. 337-339, 1989.

[6] Y. Seki, M. S. Schneider, and M. A. Meyers, "Structure and mechanical behavior of a toucan beak," Acta materialia, vol. 53, no. 20, pp. 5281-5296, 2005.

[7] D. Thompson, On Growth and Form. No. v. 2, University Press, 1968.

[8] J. Currey, Bones: Structure and Mechanics. Princeton University Press, 2002.

[9] "Biological and artificial attachment devices: Lessons for materials scientists from flies and geckos," Materials Science and Engineering: C, vol. 26, no. 8, pp. 1245$1250,2006$.

[10] M. A. Meyers, P.-Y. Chen, A. Y.-M. Lin, and Y. Seki, "Biological materials: Structure and mechanical properties," Progress in Materials Science, vol. 53, no. 1, pp. 1-206, 2008.

[11] M. A. Meyers, J. McKittrick, and P.-Y. Chen, "Structural biological materials: Critical mechanics-materials connections," Science, vol. 339, no. 6121, pp. 773-779, 2013. 
[12] P.-Y. Chen, J. McKittrick, and M. A. Meyers, "Biological materials: Functional adaptations and bioinspired designs," Progress in Materials Science, vol. 57, no. 8, pp. 1492-1704, 2012.

[13] "Biological materials: A materials science approach," Journal of the Mechanical Behavior of Biomedical Materials, vol. 4, no. 5, pp. 626-657, 2011.

[14] S. E. Naleway, J. R. Taylor, M. M. Porter, M. A. Meyers, and J. McKittrick, "Structure and mechanical properties of selected protective systems in marine organisms," Materials Science and Engineering: C, vol. 59, pp. 1143-1167, 2016.

[15] "Structure and mechanical properties of selected biological materials," Journal of the Mechanical Behavior of Biomedical Materials, vol. 1, no. 3, pp. 208-226, 2008.

[16] S. Weiner and P. M. Dove, "An overview of biomineralization processes and the problem of the vital effect," Reviews in mineralogy and geochemistry, vol. 54, no. 1, pp. 1-29, 2003.

[17] A. Miserez, T. Schneberk, C. Sun, F. W. Zok, and J. H. Waite, "The transition from stiff to compliant materials in squid beaks," Science, vol. 319, no. 5871, pp. 1816-1819, 2008.

[18] R. Marshall, D.-G. Orwin, and J. Gillespie, "Structure and biochemistry of mammalian hard keratin," Electron microscopy reviews, vol. 4, no. 1, pp. 47-83, 1991.

[19] K. W. Talmadge, K. Keegstra, W. D. Bauer, and P. Albersheim, "The structure of plant cell walls $i$. the macromolecular components of the walls of suspension-cultured sycamore cells with a detailed analysis of the pectic polysaccharides," Plant Physiology, vol. 51, no. 1, pp. 158-173, 1973 .

[20] G. M. Whitesides and M. Boncheva, "Beyond molecules: Self-assembly of mesoscopic and macroscopic components," Proceedings of the National Academy of Sciences, vol. 99 , no. 8, pp. 4769-4774, 2002.

[21] G. M. Whitesides and B. Grzybowski, "Self-assembly at all scales," Science, vol. 295, no. 5564, pp. 2418-2421, 2002.

[22] M. Kessler, N. Sottos, and S. White, "Self-healing structural composite materials," Composites Part A: applied science and manufacturing, vol. 34, no. 8, pp. 743-753, 2003.

[23] R. O. Ritchie, "The conflicts between strength and toughness," Nature materials, vol. 10, no. 11, pp. 817-822, 2011.

[24] A. Bembey, A. Bushby, A. Boyde, V. Ferguson, and M. Oyen, "Hydration effects on the micro-mechanical properties of bone," Journal of Materials Research, vol. 21, no. 08, pp. 1962-1968, 2006. 
[25] P.-Y. Chen, A. Y.-M. Lin, J. McKittrick, and M. A. Meyers, "Structure and mechanical properties of crab exoskeletons," Acta Biomaterialia, vol. 4, no. 3, pp. 587-596, 2008 .

[26] S. E. Naleway, M. M. Porter, J. McKittrick, and M. A. Meyers, "Structural design elements in biological materials: application to bioinspiration," Advanced Materials, vol. 27 , no. 37 , pp. 5455-5476, 2015 .

[27] Y. Zhao, Z. Xie, H. Gu, C. Zhu, and Z. Gu, "Bio-inspired variable structural color materials," Chemical Society Reviews, vol. 41, no. 8, pp. 3297-3317, 2012.

[28] J. A. Kluge, O. Rabotyagova, G. G. Leisk, and D. L. Kaplan, "Spider silks and their applications," Trends in biotechnology, vol. 26, no. 5, pp. 244-251, 2008.

[29] V. Ottani, D. Martini, M. Franchi, A. Ruggeri, and M. Raspanti, "Hierarchical structures in fibrillar collagens," Micron, vol. 33, no. 7, pp. 587-596, 2002.

[30] D. Raabe, P. Romano, C. Sachs, H. Fabritius, A. Al-Sawalmih, S.-B. Yi, G. Servos, and H. Hartwig, "Microstructure and crystallographic texture of the chitin-protein network in the biological composite material of the exoskeleton of the lobster homarus americanus," Materials science and engineering: A, vol. 421, no. 1, pp. 143-153, 2006.

[31] Y. Bouligand, "Twisted fibrous arrangements in biological materials and cholesteric mesophases," Tissue and Cell, vol. 4, no. 2, pp. 189-217, 1972.

[32] E. A. Zimmermann, B. Gludovatz, E. Schaible, N. K. Dave, W. Yang, M. A. Meyers, and R. O. Ritchie, "Mechanical adaptability of the bouligand-type structure in natural dermal armour," Nature communications, vol. 4, p. 2634, 2013.

[33] W. Yang, V. R. Sherman, B. Gludovatz, M. Mackey, E. A. Zimmermann, E. H. Chang, E. Schaible, Z. Qin, M. J. Buehler, R. O. Ritchie, et al., "Protective role of arapaima gigas fish scales: structure and mechanical behavior," Acta biomaterialia, vol. 10, no. 8, pp. 3599-3614, 2014.

[34] A. K. Dastjerdi and F. Barthelat, "Teleost fish scales amongst the toughest collagenous materials," Journal of the mechanical behavior of biomedical materials, vol. 52, pp. 95107,2015 .

[35] B. J. Bruet, J. Song, M. C. Boyce, and C. Ortiz, "Materials design principles of ancient fish armour," Nature materials, vol. 7, no. 9, pp. 748-756, 2008.

[36] F. Barthelat, H. Tang, P. Zavattieri, C.-M. Li, and H. Espinosa, "On the mechanics of mother-of-pearl: a key feature in the material hierarchical structure," Journal of the Mechanics and Physics of Solids, vol. 55, no. 2, pp. 306-337, 2007. 
[37] L. Tombolato, E. E. Novitskaya, P.-Y. Chen, F. A. Sheppard, and J. McKittrick, "Microstructure, elastic properties and deformation mechanisms of horn keratin," Acta biomaterialia, vol. 6, no. 2, pp. 319-330, 2010.

[38] H. Rhee, M. Horstemeyer, Y. Hwang, H. Lim, H. El Kadiri, and W. Trim, "A study on the structure and mechanical behavior of the terrapene carolina carapace: a pathway to design bio-inspired synthetic composites," Materials Science and Engineering: C, vol. 29, no. 8, pp. 2333-2339, 2009.

[39] I. H. Chen, W. Yang, and M. A. Meyers, "Leatherback sea turtle shell: A tough and flexible biological design," Acta biomaterialia, vol. 28, pp. 2-12, 2015.

[40] I. H. Chen, J. H. Kiang, V. Correa, M. I. Lopez, P.-Y. Chen, J. McKittrick, and M. A. Meyers, "Armadillo armor: mechanical testing and micro-structural evaluation," Journal of the mechanical behavior of biomedical materials, vol. 4, no. 5, pp. 713-722, 2011.

[41] I. H. Chen, W. Yang, and M. A. Meyers, "Alligator osteoderms: Mechanical behavior and hierarchical structure," Materials Science and Engineering: C, vol. 35, pp. 441$448,2014$.

[42] C. R. Jaslow, "Mechanical properties of cranial sutures," Journal of biomechanics, vol. 23, no. 4, pp. 313-321, 1990.

[43] E. Lin, Y. Li, J. C. Weaver, C. Ortiz, and M. C. Boyce, "Tunability and enhancement of mechanical behavior with additively manufactured bio-inspired hierarchical suture interfaces," Journal of Materials Research, vol. 29, no. 17, pp. 1867-1875, 2014.

[44] W. Yang, I. H. Chen, B. Gludovatz, E. A. Zimmermann, R. O. Ritchie, and M. A. Meyers, "Natural flexible dermal armor," Advanced Materials, vol. 25, no. 1, pp. 31$48,2013$.

[45] M. J. Connors, H. Ehrlich, M. Hog, C. Godeffroy, S. Araya, I. Kallai, D. Gazit, M. Boyce, and C. Ortiz, "Three-dimensional structure of the shell plate assembly of the chiton tonicella marmorea and its biomechanical consequences," Journal of structural biology, vol. 177, no. 2, pp. 314-328, 2012.

[46] U. Wegst and M. Ashby, "The mechanical efficiency of natural materials," Philosophical Magazine, vol. 84, no. 21, pp. 2167-2186, 2004.

[47] J. W. Dunlop and P. Fratzl, "Biological composites," Annual Review of Materials Research, vol. 40, pp. 1-24, 2010.

[48] M. Meyers and P. Chen, Biological Materials Science: Biological Materials, Bioinspired Materials, and Biomaterials. Cambridge University Press, 2014. 
[49] U. G. Wegst, H. Bai, E. Saiz, A. P. Tomsia, and R. O. Ritchie, "Bioinspired structural materials," Nature materials, vol. 14, no. 1, pp. 23-36, 2015.

[50] P. Fratzl and R. Weinkamer, "Nature's hierarchical materials," Progress in Materials Science, vol. 52, no. 8, pp. 1263-1334, 2007.

[51] C. Ortiz and M. C. Boyce, "Bioinspired structural materials," Science, vol. 319, no. 5866, pp. 1053-1054, 2008.

[52] M. Webster, "Merriam-webster online dictionary," 2017.

[53] A. R. Studart, "Towards high-performance bioinspired composites," Advanced Materials, vol. 24, no. 37, pp. 5024-5044, 2012.

[54] P. Ball, "Life's lessons in design," Nature, vol. 409, no. 6818, pp. 413-416, 2001.

[55] R. P. Feynman, "There's plenty of room at the bottom," Engineering and science, vol. 23 , no. 5, pp. 22-36, 1960.

[56] M. B. Frank, S. E. Naleway, T. S. Wirth, J.-Y. Jung, C. L. Cheung, F. B. Loera, S. Medina, K. N. Sato, J. R. Taylor, and J. McKittrick, "A protocol for bioinspired design: A ground sampler based on sea urchin jaws," JoVE (Journal of Visualized Experiments), no. 110, pp. e53554-e53554, 2016.

[57] M. M. Porter, Bioinspired Design: Magnetic Freeze Casting. PhD thesis, University of California, San Diego, 2014.

[58] S. Frølich, J. C. Weaver, M. N. Dean, and H. Birkedal, "Uncovering nature's design strategies through parametric modeling, multi-material 3d printing, and mechanical testing," Advanced Engineering Materials, vol. 19, no. 6, 2017.

[59] Y. Bar-Cohen, Biomimetics: biologically inspired technologies. CRC Press, 2005.

[60] Y. Bar-Cohen, Biomimetics: nature-based innovation. CRC press, 2011.

[61] J. S. Turner and R. C. Soar, "Beyond biomimicry: What termites can tell us about realizing the living building," in First International Conference on Industrialized, Intelligent Construction at Loughborough University, 2008.

[62] D. A. LaVan and J. N. Cha, "Approaches for biological and biomimetic energy conversion," Proceedings of the National Academy of Sciences, vol. 103, no. 14, pp. 5251$5255,2006$.

[63] R. J. Wood, "The first takeoff of a biologically inspired at-scale robotic insect," IEEE transactions on robotics, vol. 24, no. 2, pp. 341-347, 2008.

[64] C. Suddath, "A brief history of: Velcro.," Time Magazine, 2010. 
[65] S. Deville, E. Saiz, R. K. Nalla, and A. P. Tomsia, "Freezing as a path to build complex composites," Science, vol. 311, no. 5760, pp. 515-518, 2006.

[66] F. Bouville, E. Maire, S. Meille, B. Van de Moortèle, A. J. Stevenson, and S. Deville, "Strong, tough and stiff bioinspired ceramics from brittle constituents," Nature materials, vol. 13, no. 5, pp. 508-514, 2014.

[67] E. Munch, M. E. Launey, D. H. Alsem, E. Saiz, A. P. Tomsia, and R. O. Ritchie, "Tough, bio-inspired hybrid materials," Science, vol. 322, no. 5907, pp. 1516-1520, 2008

[68] T. Freyman, I. Yannas, and L. Gibson, "Cellular materials as porous scaffolds for tissue engineering," Progress in Materials Science, vol. 46, no. 3, pp. 273-282, 2001.

[69] H. Shin, S. Jo, and A. G. Mikos, "Biomimetic materials for tissue engineering," Biomaterials, vol. 24, no. 24, pp. 4353-4364, 2003.

[70] S. J. Hollister, "Porous scaffold design for tissue engineering," Nature materials, vol. 4, no. 7, pp. 518-524, 2005.

[71] S. Deville, "Freeze-casting of porous ceramics: a review of current achievements and issues," Advanced Engineering Materials, vol. 10, no. 3, pp. 155-169, 2008.

[72] H. Lee, B. P. Lee, and P. B. Messersmith, "A reversible wet/dry adhesive inspired by mussels and geckos," Nature, vol. 448, no. 7151, pp. 338-341, 2007.

[73] L. F. Boesel, C. Greiner, E. Arzt, and A. Del Campo, "Gecko-inspired surfaces: a path to strong and reversible dry adhesives," Advanced Materials, vol. 22, no. 19, pp. 2125-2137, 2010.

[74] A. Lazaris, S. Arcidiacono, Y. Huang, J.-F. Zhou, F. Duguay, N. Chretien, E. A. Welsh, J. W. Soares, and C. N. Karatzas, "Spider silk fibers spun from soluble recombinant silk produced in mammalian cells," science, vol. 295, no. 5554, pp. 472-476, 2002 .

[75] A. Seidel, O. Liivak, and L. W. Jelinski, "Artificial spinning of spider silk," Macromolecules, vol. 31, no. 19, pp. 6733-6736, 1998.

[76] X.-X. Xia, Z.-G. Qian, C. S. Ki, Y. H. Park, D. L. Kaplan, and S. Y. Lee, "Native-sized recombinant spider silk protein produced in metabolically engineered escherichia coli results in a strong fiber," Proceedings of the National Academy of Sciences, vol. 107, no. 32 , pp. 14059-14063, 2010.

[77] B. Bhushan, "Bioinspired structured surfaces," Langmuir, vol. 28, no. 3, pp. 1698$1714,2012$. 
[78] A. Solga, Z. Cerman, B. F. Striffler, M. Spaeth, and W. Barthlott, "The dream of staying clean: Lotus and biomimetic surfaces," Bioinspiration 83 biomimetics, vol. 2, no. 4, p. S126, 2007.

[79] X.-M. Li, D. Reinhoudt, and M. Crego-Calama, "What do we need for a superhydrophobic surface? a review on the recent progress in the preparation of superhydrophobic surfaces," Chemical Society Reviews, vol. 36, no. 8, pp. 1350-1368, 2007.

[80] A. Klaus, V. Kulasekera, and V. Schawaroch, "Three-dimensional visualization of insect morphology using confocal laser scanning microscopy," Journal of Microscopy, vol. 212 , no. 2 , pp. 107-121, 2003.

[81] O. Betz, U. Wegst, D. Weide, M. Heethoff, L. Helfen, W.-K. LEE, and P. Cloetens, "Imaging applications of synchrotron x-ray phase-contrast microtomography in biological morphology and biomaterials science. i. general aspects of the technique and its advantages in the analysis of millimetre-sized arthropod structure," Journal of Microscopy, vol. 227, no. 1, pp. 51-71, 2007.

[82] S. R. Stock, Microcomputed tomography: methodology and applications. CRC press, 2008 .

[83] G. G. Poludniowski, "Calculation of x-ray spectra emerging from an x-ray tube. part ii. x-ray production and filtration in x-ray targets," Medical physics, vol. 34, no. 6, pp. 2175-2186, 2007.

[84] G. N. Hounsfield, "Computerized transverse axial scanning (tomography): Part 1. description of system," The British journal of radiology, vol. 46, no. 552, pp. 10161022, 1973.

[85] P. Tafforeau, R. Boistel, E. Boller, A. Bravin, M. Brunet, Y. Chaimanee, P. Cloetens, M. Feist, J. Hoszowska, J.-J. Jaeger, et al., "Applications of x-ray synchrotron microtomography for non-destructive 3d studies of paleontological specimens," Applied Physics A, vol. 83, no. 2, pp. 195-202, 2006.

[86] A. Scott, I. Sinclair, S. M. Spearing, M. N. Mavrogordato, and W. Hepples, "Influence of voids on damage mechanisms in carbon/epoxy composites determined via high resolution computed tomography," Composites Science and Technology, vol. 90, pp. 147-153, 2014.

[87] V. Cnudde and M. N. Boone, "High-resolution x-ray computed tomography in geosciences: A review of the current technology and applications," Earth-Science Reviews, vol. 123, pp. 1-17, 2013.

[88] P. M. O'Connor, J. J. Sertich, N. J. Stevens, E. M. Roberts, M. D. Gottfried, T. L. Hieronymus, Z. A. Jinnah, R. Ridgely, S. E. Ngasala, and J. Temba, "The evolution of 
mammal-like crocodyliforms in the cretaceous period of gondwana," Nature, vol. 466, no. 7307, pp. 748-751, 2010.

[89] J. Hsieh, B. Nett, Z. Yu, K. Sauer, J.-B. Thibault, and C. A. Bouman, "Recent advances in ct image reconstruction," Current Radiology Reports, vol. 1, no. 1, pp. 39$51,2013$.

[90] E. Maire and P. Withers, "Quantitative x-ray tomography," International materials reviews, vol. 59, no. 1, pp. 1-43, 2014.

[91] A. C. Kak and M. Slaney, Principles of computerized tomographic imaging. SIAM, 2001.

[92] J. Hsieh, Computed tomography: principles, design, artifacts, and recent advances, vol. 114. SPIE press, 2003.

[93] J. Hsieh, "Computed tomography: principles, design, artifacts, and recent advances," SPIE Bellingham, WA, 2009.

[94] R. Garwood, Tomographic reconstruction of Carboniferous Arthropods. PhD thesis, Imperial College, London, 2011.

[95] G. Davis and J. Elliott, "Artefacts in x-ray microtomography of materials," Materials science and technology, vol. 22, no. 9, pp. 1011-1018, 2006.

[96] S. Akin and A. Kovscek, "Computed tomography in petroleum engineering research," Geological Society, London, Special Publications, vol. 215, no. 1, pp. 23-38, 2003.

[97] J. Sijbers and A. Postnov, "Reduction of ring artefacts in high resolution micro-ct reconstructions," Physics in medicine and biology, vol. 49, no. 14, p. N247, 2004.

[98] B. D. Metscher, "Microct for comparative morphology: simple staining methods allow high-contrast 3d imaging of diverse non-mineralized animal tissues," BMC physiology, vol. 9 , no. 1 , p. $11,2009$.

[99] Y. M. Staedler, D. Masson, and J. Schönenberger, "Plant tissues in 3d via x-ray tomography: simple contrasting methods allow high resolution imaging," PloS one, vol. 8, no. 9, p. e75295, 2013.

[100] B. D. Metscher, "Microct for developmental biology: A versatile tool for high-contrast 3d imaging at histological resolutions," Developmental dynamics, vol. 238, no. 3, pp. 632-640, 2009.

[101] B. D. Metscher, "X-ray microtomographic imaging of intact vertebrate embryos," Cold Spring Harbor Protocols, vol. 2011, no. 12, pp. pdb-prot067033, 2011. 
[102] I. Zanette, G. Daghfous, T. Weitkamp, B. Gillet, D. Adriaens, M. Langer, P. Cloetens, L. Helfen, A. Bravin, F. Peyrin, et al., "Looking inside marine organisms with magnetic resonance and x-ray imaging," Imaging marine life, pp. 122-184, 2013.

[103] S. Wilkins, T. E. Gureyev, D. Gao, A. Pogany, and A. Stevenson, "Phase-contrast imaging using polychromatic hard x-rays," Nature, vol. 384, no. 6607, p. 335, 1996.

[104] S. Zabler, P. Cloetens, J.-P. Guigay, J. Baruchel, and M. Schlenker, "Optimization of phase contrast imaging using hard x rays," Review of Scientific Instruments, vol. 76, no. 7, p. $073705,2005$.

[105] T. Weitkamp, A. Diaz, C. David, F. Pfeiffer, M. Stampanoni, P. Cloetens, and E. Ziegler, "X-ray phase imaging with a grating interferometer," Optics express, vol. 13, no. 16, pp. 6296-6304, 2005.

[106] A. Tkachuk, F. Duewer, H. Cui, M. Feser, S. Wang, and W. Yun, "X-ray computed tomography in zernike phase contrast mode at $8 \mathrm{kev}$ with $50-\mathrm{nm}$ resolution using $\mathrm{cu}$ rotating anode x-ray source," Zeitschrift für Kristallographie-Crystalline Materials, vol. 222 , no. $11 / 2007$, pp. $650-655,2007$.

[107] C. K. Hagen, P. Maghsoudlou, G. Totonelli, P. C. Diemoz, M. Endrizzi, L. Rigon, R.-H. Menk, F. Arfelli, D. Dreossi, E. Brun, et al., "High contrast microstructural visualization of natural acellular matrices by means of phase-based x-ray tomography," Scientific reports, vol. 5, p. 18156, 2015.

[108] J.-Y. Jung, S. E. Naleway, N. A. Yaraghi, S. Herrera, V. R. Sherman, E. A. Bushong, M. H. Ellisman, D. Kisailus, and J. McKittrick, "Structural analysis of the tongue and hyoid apparatus in a woodpecker," Acta biomaterialia, vol. 37, pp. 1-13, 2016.

[109] J. Taylor and S. Patek, "Ritualized fighting and biological armor: the impact mechanics of the mantis shrimp's telson," Journal of Experimental Biology, vol. 213, no. 20, pp. 3496-3504, 2010.

[110] M. E. Launey, P.-Y. Chen, J. McKittrick, and R. O. Ritchie, "Mechanistic aspects of the fracture toughness of elk antler bone," Acta biomaterialia, vol. 6, no. 4, pp. 1505$1514,2010$.

[111] W. Yang, S. E. Naleway, M. M. Porter, M. A. Meyers, and J. McKittrick, "The armored carapace of the boxfish," Acta biomaterialia, vol. 23, pp. 1-10, 2015.

[112] C.-Y. Sun and P.-Y. Chen, "Structural design and mechanical behavior of alligator (alligator mississippiensis) osteoderms," Acta biomaterialia, vol. 9, no. 11, pp. 90499064, 2013. 
[113] M. M. Porter, E. Novitskaya, A. B. Castro-Ceseña, M. A. Meyers, and J. McKittrick, "Highly deformable bones: unusual deformation mechanisms of seahorse armor," Acta biomaterialia, vol. 9, no. 6, pp. 6763-6770, 2013.

[114] M. M. Porter, D. Adriaens, R. L. Hatton, M. A. Meyers, and J. McKittrick, "Why the seahorse tail is square," Science, vol. 349, no. 6243, pp. 46-53, 2015.

[115] Y. Seki, S. G. Bodde, and M. A. Meyers, "Toucan and hornbill beaks: a comparative study," Acta Biomaterialia, vol. 6, no. 2, pp. 331-343, 2010.

[116] L. Wen, J. C. Weaver, and G. V. Lauder, "Biomimetic shark skin: design, fabrication and hydrodynamic function," Journal of Experimental Biology, vol. 217, no. 10, pp. 1656-1666, 2014.

[117] P. J. Withers and M. Preuss, "Fatigue and damage in structural materials studied by x-ray tomography," annual review of materials research, vol. 42, pp. 81-103, 2012.

[118] G. Tan, P. Xu, L. B. Lawson, J. He, L. C. Freytag, J. D. Clements, and V. T. John, "Hydration effects on skin microstructure as probed by high-resolution cryo-scanning electron microscopy and mechanistic implications to enhanced transcutaneous delivery of biomacromolecules," Journal of pharmaceutical sciences, vol. 99, no. 2, pp. 730-740, 2010 .

[119] H. D. Barth, E. A. Zimmermann, E. Schaible, S. Y. Tang, T. Alliston, and R. O. Ritchie, "Characterization of the effects of x-ray irradiation on the hierarchical structure and mechanical properties of human cortical bone," Biomaterials, vol. 32, no. 34, pp. 8892-8904, 2011.

[120] T. Lowe, R. J. Garwood, T. J. Simonsen, R. S. Bradley, and P. J. Withers, "Metamorphosis revealed: time-lapse three-dimensional imaging inside a living chrysalis," Journal of the Royal Society Interface, vol. 10, no. 84, p. 20130304, 2013.

[121] S. M. Walker, D. A. Schwyn, R. Mokso, M. Wicklein, T. Müller, M. Doube, M. Stampanoni, H. G. Krapp, and G. K. Taylor, "In vivo time-resolved microtomography reveals the mechanics of the blowfly flight motor," PLoS Biol, vol. 12, no. 3, p. e1001823, 2014

[122] A. T. Jang, J. D. Lin, Y. Seo, S. Etchin, A. Merkle, K. Fahey, and S. P. Ho, "In situ compressive loading and correlative noninvasive imaging of the bone-periodontal ligament-tooth fibrous joint," JoVE (Journal of Visualized Experiments), no. 85, pp. e51147-e51147, 2014.

[123] W. Wiest, S. Zabler, A. Rack, C. Fella, A. Balles, K. Nelson, R. Schmelzeisen, and R. Hanke, "In situ microradioscopy and microtomography of fatigue-loaded dental 
two-piece implants," Journal of synchrotron radiation, vol. 22, no. 6, pp. 1492-1497, 2015.

[124] B. Patterson, N. Cordes, K. Henderson, J. Mertens, A. Clarke, B. Hornberger, A. Merkle, S. Etchin, A. Tkachuk, M. Leibowitz, et al., "In situ laboratory-based transmission x-ray microscopy and tomography of material deformation at the nanoscale," Experimental Mechanics, vol. 56, no. 9, pp. 1585-1597, 2016.

[125] H. Gao, B. Ji, I. L. Jäger, E. Arzt, and P. Fratzl, "Materials become insensitive to flaws at nanoscale: lessons from nature," Proceedings of the national Academy of Sciences, vol. 100, no. 10, pp. 5597-5600, 2003.

[126] C. A. Schneider, W. S. Rasband, K. W. Eliceiri, et al., "Nih image to imagej: 25 years of image analysis," Nat methods, vol. 9, no. 7, pp. 671-675, 2012.

[127] S. Nishikawa and S. Kikuchi, "Diffraction of cathode rays by mica," Nature, vol. 121, pp. 1019-1020, 1928.

[128] S. Nishikawa and S. Kikuchi, "The diffraction of cathode rays by calcite," Proceedings of the Imperial Academy, vol. 4, no. 8, pp. 475-477, 1928.

[129] M. Alam, M. Blackman, and D. Pashley, "High-angle kikuchi patterns," in Proceedings of the Royal Society of London A: Mathematical, Physical and Engineering Sciences, vol. 221, pp. 224-242, The Royal Society, 1954.

[130] D. Dingley, "Diffraction from sub-micron areas using electron backscattering in a scanning electron microscope," Scanning electron microscopy, vol. 2, pp. 569-575, 1984

[131] R. A. Schwarzer, "Automated crystal lattice orientation mapping using a computercontrolled sem," Micron, vol. 28, no. 3, pp. 249-265, 1997.

[132] A. J. Schwartz, M. Kumar, B. L. Adams, and D. P. Field, Electron backscatter diffraction in materials science, vol. 2. Springer, 2009.

[133] M. Ebner, S. Piazolo, F. Renard, and D. Koehn, "Stylolite interfaces and surrounding matrix material: Nature and role of heterogeneities in roughness and microstructural development," Journal of Structural Geology, vol. 32, no. 8, pp. 1070-1084, 2010.

[134] M. Cusack, "Biomineral electron backscatter diffraction for palaeontology," Palaeontology, vol. 59, no. 2, pp. 171-179, 2016.

[135] W. W. Schmahl, E. Griesshaber, R. Neuser, A. Lenze, J. Reinhart, and U. Brand, "The microstructure of the fibrous layer of terebratulide brachiopod shell calcite," European Journal of Mineralogy, vol. 16, no. 4, pp. 693-697, 2004. 
[136] M. Suzuki, J. Kameda, T. Sasaki, K. Saruwatari, H. Nagasawa, and T. Kogure, "Characterization of the multilayered shell of a limpet, lottia kogamogai (mollusca: Patellogastropoda), using sem-ebsd and fib-tem techniques," Journal of structural biology, vol. 171, no. 2, pp. 223-230, 2010.

[137] A. Pérez-Huerta, Y. Dauphin, J. P. Cuif, and M. Cusack, "High resolution electron backscatter diffraction (ebsd) data from calcite biominerals in recent gastropod shells," Micron, vol. 42, no. 3, pp. 246-251, 2011.

[138] E. DiMasi and L. B. Gower, Biomineralization sourcebook: characterization of biominerals and biomimetic materials. CRC Press, 2014.

[139] M. M. Nowell, R. A. Witt, and B. True, "Ebsd sample preparation: Techniques, tips, and tricks," Microscopy and Microanalysis, vol. 11, no. S02, pp. 504-505, 2005.

[140] A. Pérez-Huerta and M. Cusack, "Optimizing electron backscatter diffraction of carbonate biominerals - resin type and carbon coating," Microscopy and Microanalysis, vol. 15, no. 03, pp. 197-203, 2009.

[141] E. Mahway, "Oim user's manual," EDAX-TSL, New Jersey, 2005.

[142] R. F. Cook, "Probing the nanoscale," Science, vol. 328, no. 5975, pp. 183-184, 2010.

[143] D. M. Ebenstein and L. A. Pruitt, "Nanoindentation of biological materials," Nano Today, vol. 1, no. 3, pp. 26-33, 2006.

[144] A. Fischer-Cripps and D. Nicholson, "Nanoindentation. mechanical engineering series," Applied Mechanics Reviews, vol. 57, p. 12, 2004.

[145] J. L. Cuy, A. B. Mann, K. J. Livi, M. F. Teaford, and T. P. Weihs, "Nanoindentation mapping of the mechanical properties of human molar tooth enamel," Archives of Oral Biology, vol. 47, no. 4, pp. 281-291, 2002.

[146] H. C. Lichtenegger, T. Schöberl, M. H. Bartl, H. Waite, and G. D. Stucky, "High abrasion resistance with sparse mineralization: copper biomineral in worm jaws," Science, vol. 298, no. 5592, pp. 389-392, 2002.

[147] K. Zhang, F. Si, H. Duan, and J. Wang, "Microstructures and mechanical properties of silks of silkworm and honeybee," Acta biomaterialia, vol. 6, no. 6, pp. 2165-2171, 2010 .

[148] P. Allison, M. Chandler, R. Rodriguez, B. Williams, R. Moser, C. Weiss, A. Poda, B. Lafferty, A. Kennedy, J. Seiter, et al., "Mechanical properties and structure of the biological multilayered material system, atractosteus spatula scales," Acta biomaterialia, vol. 9, no. 2, pp. 5289-5296, 2013. 
[149] B. Bruet, H. Qi, M. Boyce, R. Panas, K. Tai, L. Frick, and C. Ortiz, "Nanoscale morphology and indentation of individual nacre tablets from the gastropod mollusc trochus niloticus," Journal of Materials Research, vol. 20, no. 09, pp. 2400-2419, 2005.

[150] C. Klapperich, K. Komvopoulos, and L. Pruitt, "Nanomechanical properties of polymers determined from nanoindentation experiments," Journal of tribology, vol. 123, no. 3, pp. 624-631, 2001.

[151] A. Bembey, M. Oyen, A. Bushby, and A. Boyde, "Viscoelastic properties of bone as a function of hydration state determined by nanoindentation," Philosophical Magazine, vol. 86, no. 33-35, pp. 5691-5703, 2006.

[152] M. Oyen, "Nanoindentation of biological and biomimetic materials," Experimental Techniques, vol. 37, no. 1, pp. 73-87, 2013.

[153] W. C. Oliver and G. M. Pharr, "Measurement of hardness and elastic modulus by instrumented indentation: Advances in understanding and refinements to methodology," Journal of materials research, vol. 19, no. 01, pp. 3-20, 2004.

[154] W. C. Oliver and G. M. Pharr, "An improved technique for determining hardness and elastic modulus using load and displacement sensing indentation experiments," Journal of materials research, vol. 7, no. 06, pp. 1564-1583, 1992.

[155] F. Rengier, A. Mehndiratta, H. von Tengg-Kobligk, C. M. Zechmann, R. Unterhinninghofen, H.-U. Kauczor, and F. L. Giesel, "3d printing based on imaging data: review of medical applications," International journal of computer assisted radiology and surgery, vol. 5, no. 4, pp. 335-341, 2010.

[156] H. Lipson and M. Kurman, Fabricated: The new world of 3D printing. John Wiley \& Sons, 2013.

[157] H. N. Chia and B. M. Wu, "Recent advances in 3d printing of biomaterials," Journal of biological engineering, vol. 9, no. 1, p. 4, 2015.

[158] J. A. Lewis and B. Y. Ahn, "Device fabrication: Three-dimensional printed electronics," Nature, vol. 518, no. 7537, pp. 42-43, 2015.

[159] Y. L. Kong, I. A. Tamargo, H. Kim, B. N. Johnson, M. K. Gupta, T.-W. Koh, H.-A. Chin, D. A. Steingart, B. P. Rand, and M. C. McAlpine, "3d printed quantum dot light-emitting diodes," Nano letters, vol. 14, no. 12, pp. 7017-7023, 2014.

[160] A. R. Studart, "Additive manufacturing of biologically-inspired materials," Chemical Society Reviews, vol. 45, no. 2, pp. 359-376, 2016.

[161] G. X. Gu, I. Su, S. Sharma, J. L. Voros, Z. Qin, and M. J. Buehler, "Threedimensional-printing of bio-inspired composites," Journal of biomechanical engineering, vol. 138, no. 2, p. 021006, 2016. 
[162] Z. Qin, B. G. Compton, J. A. Lewis, and M. J. Buehler, "Structural optimization of 3d-printed synthetic spider webs for high strength," Nature communications, vol. 6, p. $7038,2015$.

[163] L. S. Dimas, G. H. Bratzel, I. Eylon, and M. J. Buehler, "Tough composites inspired by mineralized natural materials: computation, 3d printing, and testing," Advanced Functional Materials, vol. 23, no. 36, pp. 4629-4638, 2013.

[164] J. J. Martin, B. E. Fiore, and R. M. Erb, "Designing bioinspired composite reinforcement architectures via $3 \mathrm{~d}$ magnetic printing," Nature communications, vol. 6, p. 8641, 2015 .

[165] J. Kingdon, D. Happold, T. Butynski, M. Hoffmann, M. Happold, and J. Kalina, Mammals of Africa. No. v. 1-6, Bloomsbury Publishing, 2013.

[166] R. Piper, Extraordinary animals: An encyclopedia of curious and unusual animals. Greenwood Publishing Group, 2007.

[167] J. Kingdon, East African Mammals: pt. A. Insectivores and bats, vol. 2. Academic press, 1974.

[168] W. T. Stanley, L. W. Robbins, J. M. Malekani, S. G. Mbalitini, D. A. Migurimu, J. C. Mukinzi, J. Hulselmans, V. Prévot, E. Verheyen, R. Hutterer, et al., "A new hero emerges: another exceptional mammalian spine and its potential adaptive significance," Biology letters, vol. 9, no. 5, p. 20130486, 2013.

[169] J. A. Allen, H. Lang, and J. P. Chapin, The skeletal characters of Scutisorex Thomas. order of the Trustees, American Museum of Natural History, 1917.

[170] A. Ahmed and M. Klima, "Zur entwicklung und funktion der lendenwirbelsäule bei der panzerspitzmaus scutisorex somereni (thomas, 1910)," Z. Saugetierkd., vol. 43, pp. 1-17, 1978.

[171] D. M. Cullinane, D. Aleper, and J. E. Bertram, "The functional and biomechanical modifications of the spine of scutisorex somereni, the hero shrew: skeletal scaling relationships," Journal of Zoology, vol. 244, no. 3, pp. 447-452, 1998.

[172] D. M. Cullinane and D. Aleper, "The functional and biomechanical modifications of the spine of scutisorex somereni, the hero shrew: spinal musculature," Journal of Zoology, vol. 244, no. 3, pp. 453-458, 1998.

[173] D. M. Cullinane and J. E. Bertram, "The mechanical behaviour of a novel mammalian intervertebral joint," Journal of anatomy, vol. 197, no. 4, pp. 627-634, 2000.

[174] T. Stankowich, "Armed and dangerous: predicting the presence and function of defensive weaponry in mammals," Adaptive Behavior, vol. 20, no. 1, pp. 32-43, 2012. 
[175] S. Krauss, E. Monsonego-Ornan, E. Zelzer, P. Fratzl, and R. Shahar, "Mechanical function of a complex three-dimensional suture joining the bony elements in the shell of the red-eared slider turtle," Advanced Materials, vol. 21, no. 4, pp. 407-412, 2009.

[176] E. Denton and J. Gilpin-Brown, "The buoyancy of the cuttlefish, Sepia officinalis (1.)," Journal of the Marine Biological Association of the United Kingdom, vol. 41, no. 02, pp. 319-342, 1961.

[177] J. Birchall and N. Thomas, "On the architecture and function of cuttlefish bone," Journal of Materials Science, vol. 18, no. 7, pp. 2081-2086, 1983.

[178] R. Hackman and M. Goldberg, "Studies on chitin vi. the nature of $\alpha$ - and $\beta$ - chitins," Australian Journal of Biological Sciences, vol. 18, no. 4, pp. 935-946.

[179] N. Okafor, "Isolation of chitin from the shell of the cuttlefish, Sepia officinalis 1.," Biochimica et Biophysica Acta (BBA)-Mucoproteins and Mucopolysaccharides, vol. 101, no. 2, pp. 193-200, 1965.

[180] Y. Dauphin and F. Marin, "The compositional analysis of recent cephalopod shell carbohydrates by fourier transform infrared spectrometry and high performance anion exchange-pulsed amperometric detection," Experientia, vol. 51, no. 3, pp. 278-283, 1995.

[181] M. Florek, E. Fornal, P. Gómez-Romero, E. Zieba, W. Paszkowicz, J. Lekki, J. Nowak, and A. Kuczumow, "Complementary microstructural and chemical analyses of Sepia officinalis endoskeleton," Materials Science and Engineering: C, vol. 29, no. 4, pp. 1220-1226, 2009.

[182] C. Le Pabic, A. Marie, B. Marie, A. Percot, L. Bonnaud-Ponticelli, P. J. Lopez, and G. Luquet, "First proteomic analyses of the dorsal and ventral parts of the Sepia officinalis cuttlebone," Journal of Proteomics, vol. 150, pp. 63-73, 2017.

[183] K. Bandel and S. Boletzky, "Comparative study of the structure, development and morphological relationships of chambered cephalopod shells," Veliger, vol. 21, no. 3, pp. 313-354, 1979.

[184] A. G. Checa, J. H. Cartwright, I. Sánchez-Almazo, J. P. Andrade, and F. Ruiz-Raya, "The cuttlefish Sepia officinalis (sepiidae, cephalopoda) constructs cuttlebone from a liquid-crystal precursor," Scientific reports, vol. 5, p. 11513, 2015.

[185] E. Denton, J. Gilpin-Brown, and J. Howarth, "The osmotic mechanism of the cuttlebone," Journal of the Marine Biological Association of the United Kingdom, vol. 41, no. 02, pp. 351-363, 1961. 
[186] P. D. Ward and S. Von Boletzky, "Shell implosion depth and implosion morphologies in three species of Sepia (cephalopoda) from the mediterranean sea," Journal of the Marine Biological Association of the United Kingdom, vol. 64, no. 04, pp. 955-966, 1984.

[187] P. Neige and S. Boletzky, "Morphometrics of the shell of three Sepia species (mollusca: Cephalopoda): intra-and interspecific variation," Zoologische Beitrage, vol. 38, pp. 137-156, 1997.

[188] D. Milovac, G. G. Ferrer, M. Ivankovic, and H. Ivankovic, "Pcl-coated hydroxyapatite scaffold derived from cuttlefish bone: Morphology, mechanical properties and bioactivity," Materials Science and Engineering: C, vol. 34, pp. 437-445, 2014.

[189] J. J. A. Appellöf, Die schalen von Sepia, Spirula und Nautilus: Studien über den bau und das wachsthum, vol. 25. PA Norsted \& söner, 1893.

[190] K. Tanabe, Y. Fukuda, and Y. Ohtsuka, "New chamber formation in the cuttlefish Sepia esculenta," Venus, vol. 44, no. 1, pp. 55-67, 1985.

[191] D. Gower and J. Vincent, "The mechanical design of the cuttlebone and its bathymetric implications," Biomimetics, vol. 4, pp. 37-58, 1996.

[192] K. M. Sherrard, "Cuttlebone morphology limits habitat depth in eleven species of Sepia (cephalopoda: Sepiidae)," The Biological Bulletin, vol. 198, no. 3, pp. 404-414, 2000.

[193] M. A. Gutowska, F. Melzner, H. O. Pörtner, and S. Meier, "Cuttlebone calcification increases during exposure to elevated seawater pco2 in the cephalopod Sepia officinalis," Marine Biology, vol. 157, no. 7, pp. 1653-1663, 2010.

[194] V. Bettencourt and A. Guerra, "Age studies based on daily growth increments in statoliths and growth lamellae in cuttlebone of cultured Sepia officinalis," Marine Biology, vol. 139, no. 2, pp. 327-334, 2001.

[195] W. Ogasawara, W. Shenton, S. A. Davis, and S. Mann, "Template mineralization of ordered macroporous chitin-silica composites using a cuttlebone-derived organic matrix," Chemistry of Materials, vol. 12, no. 10, pp. 2835-2837, 2000.

[196] J. Rocha, A. Lemos, S. Agathopoulos, P. Valério, S. Kannan, F. Oktar, and J. Ferreira, "Scaffolds for bone restoration from cuttlefish," Bone, vol. 37, no. 6, pp. 850-857, 2005.

[197] Ö. S. Yıldırım, Z. Okumuş, M. Kizilkaya, Y. Özdemir, R. Durak, and A. Okur, "Comparative quantitative analysis of sodium, magnesium, potassium and calcium in healthy cuttlefish backbone and non-pathological human elbow bone," Canadian Journal of Analytical Sciences and Spectroscopy, vol. 52, pp. 270-275, 2007. 
[198] H. Ivankovic, G. G. Ferrer, E. Tkalcec, S. Orlic, and M. Ivankovic, "Preparation of highly porous hydroxyapatite from cuttlefish bone," Journal of Materials Science: Materials in Medicine, vol. 20, no. 5, pp. 1039-1046, 2009.

[199] H. Ivankovic, E. Tkalcec, S. Orlic, G. G. Ferrer, and Z. Schauperl, "Hydroxyapatite formation from cuttlefish bones: kinetics," Journal of Materials Science: Materials in Medicine, vol. 21, no. 10, pp. 2711-2722, 2010.

[200] A. Kasioptas, T. Geisler, C. V. Putnis, C. Perdikouri, and A. Putnis, "Crystal growth of apatite by replacement of an aragonite precursor," Journal of Crystal Growth, vol. 312, no. 16, pp. 2431-2440, 2010.

[201] J. Rocha, A. Lemos, S. Kannan, S. Agathopoulos, and J. Ferreira, "Hydroxyapatite scaffolds hydrothermally grown from aragonitic cuttlefish bones," Journal of Materials Chemistry, vol. 15, no. 47, pp. 5007-5011, 2005.

[202] J. Rocha, A. Lemos, S. Agathopoulos, S. Kannan, P. Valerio, and J. Ferreira, "Hydrothermal growth of hydroxyapatite scaffolds from aragonitic cuttlefish bones," Journal of Biomedical Materials Research Part A, vol. 77, no. 1, pp. 160-168, 2006.

[203] S. Kannan, J. Rocha, S. Agathopoulos, and J. Ferreira, "Fluorine-substituted hydroxyapatite scaffolds hydrothermally grown from aragonitic cuttlefish bones," Acta Biomaterialia, vol. 3, no. 2, pp. 243-249, 2007.

[204] P. Sarin, S.-J. Lee, Z. D. Apostolov, and W. M. Kriven, "Porous biphasic calcium phosphate scaffolds from cuttlefish bone," Journal of the American Ceramic Society, vol. 94 , no. 8, pp. 2362-2370, 2011.

[205] E. Battistella, S. Mele, I. Foltran, I. G. Lesci, N. Roveri, P. Sabatino, and L. Rimondini, "Cuttlefish bone scaffold for tissue engineering: a novel hydrothermal transformation, chemical-physical, and biological characterization," J Appl Biomater Funct Mater, vol. 10, no. 2, pp. 99-106, 2012.

[206] S. Poompradub, Y. Ikeda, Y. Kokubo, and T. Shiono, "Cuttlebone as reinforcing filler for natural rubber," European Polymer Journal, vol. 44, no. 12, pp. 4157-4164, 2008.

[207] S. Shang, K.-L. Chiu, M. C. W. Yuen, and S. Jiang, "The potential of cuttlebone as reinforced filler of polyurethane," Composites Science and Technology, vol. 93, pp. 1722,2014 .

[208] P. Kamalbabu and G. M. Kumar, "Effects of particle size on tensile properties of marine coral reinforced polymer composites," Procedia Materials Science, vol. 5, pp. 802808, 2014. 
[209] K. Periasamy and G. Mohankumar, "Sea coral-derived cuttlebone reinforced epoxy composites: Characterization and tensile properties evaluation with mathematical models," Journal of Composite Materials, vol. 50, no. 6, pp. 807-823, 2016.

[210] C. F. Jasso-Gastinel, S. G. Enriquez, J. Flores, I. Reyes-González, and E. M. Mijares, "Acrylic bone cements modified with bioactive filler," in Macromolecular symposia, vol. 283, pp. 159-166, Wiley Online Library, 2009.

[211] S. García-Enriquez, H. Guadarrama, I. Reyes-González, E. Mendizábal, C. JassoGastinel, B. García-Enriquez, D. Rembao-Bojórquez, and C. Pane-Pianese, "Mechanical performance and in vivo tests of an acrylic bone cement filled with bioactive sepia officinalis cuttlebone," Journal of Biomaterials Science, Polymer Edition, vol. 21, no. 1, pp. 113-125, 2010.

[212] E. Culverwell, S. C. Wimbush, and S. R. Hall, "Biotemplated synthesis of an ordered macroporous superconductor with high critical current density using a cuttlebone template," Chemical Communications, no. 9, pp. 1055-1057, 2008.

[213] J. Cadman, S. Zhou, Y. Chen, and Q. Li, "Cuttlebone: characterisation, application and development of biomimetic materials," Journal of Bionic Engineering, vol. 9, no. 3, pp. 367-376, 2012.

[214] J. Cadman, S. Zhou, Y. Chen, W. Li, R. Appleyard, and Q. Li, "Characterization of cuttlebone for a biomimetic design of cellular structures," Acta Mechanica Sinica, vol. 26 , no. 1, pp. 27-35, 2010.

[215] C. Le Pabic, M. Rousseau, L. Bonnaud-Ponticelli, and S. von Boletzky, "Overview of the shell development of the common cuttlefish Sepia officinalis during early-life stages," Vie et Milieu-Life and Environment, vol. 66, no. 1, pp. 35-42, 2016.

[216] J. Cadman, C.-C. Chang, J. Chen, Y. Chen, S. Zhou, W. Li, and Q. Li, "Bioinspired lightweight cellular materials-understanding effects of natural variation on mechanical properties," Materials Science and Engineering: C, vol. 33, no. 6, pp. 3146-3152, 2013.

[217] A. Limaye, "Drishti: a volume exploration and presentation tool," in Proc Spie, vol. 8506, pp. 85060X-85060X, 2012.

[218] P. Ré and L. Narciso, "Growth and cuttlebone microstructure of juvenile cuttlefish, Sepia officinalis 1., under controlled conditions," Journal of experimental marine biology and ecology, vol. 177, no. 1, pp. 73-78, 1994.

[219] M. A. Gutowska, H. O. Pörtner, and F. Melzner, "Growth and calcification in the cephalopod Sepia officinalis under elevated seawater pco2," Marine Ecology Progress Series, vol. 373, pp. 303-309, 2008. 
[220] C. Li and P. K.-S. Tam, "An iterative algorithm for minimum cross entropy thresholding," Pattern Recognition Letters, vol. 19, no. 8, pp. 771-776, 1998.

[221] M. Doube, M. M. Kłosowski, I. Arganda-Carreras, F. P. Cordelières, R. P. Dougherty, J. S. Jackson, B. Schmid, J. R. Hutchinson, and S. J. Shefelbine, "Bonej: free and extensible bone image analysis in imagej," Bone, vol. 47, no. 6, pp. 1076-1079, 2010.

[222] M. Cusack and P. Chung, "Crystallographic orientation of cuttlebone shield determined by electron backscatter diffraction," Jom, vol. 66, no. 1, pp. 139-142, 2014.

[223] L. North, "3d_print_mount_nikonxt225.stl," 32017.

[224] L. A. Doguzhaeva and E. Dunca, "Siphonal zone structure in the cuttlebone of Sepia officinalis," Swiss Journal of Palaeontology, vol. 134, no. 2, pp. 167-176, 2015.

[225] E. Denton and J. n. Gilpin-Brown, "The distribution of gas and liquid within the cuttlebone," Journal of the Marine Biological Association of the United Kingdom, vol. 41, no. 02, pp. 365-381, 1961.

[226] S. Boletzky, "Sepia officinalis," Cephalopod Life Cycles. Academic Press, London, 1983.

[227] A. M. Turing, "The chemical basis of morphogenesis," Bulletin of mathematical biology, vol. 52, no. 1-2, pp. 153-197, 1990.

[228] S. Kondo and R. Asai, "A reaction-diffusion wave on the skin of the marine angelfish pomacanthus," Nature, vol. 376, no. 6543, p. 765, 1995.

[229] K. Bandel, "Cephalopod shell structure and general mechanisms of shell formation," Skeletal biomineralization: patterns, processes and evolutionary trends, pp. 97-115, 1990.

[230] S. Weiner and L. Addadi, "Design strategies in mineralized biological materials," Journal of Materials Chemistry, vol. 7, no. 5, pp. 689-702, 1997.

[231] M. L. Oyen, V. L. Ferguson, A. K. Bembey, A. J. Bushby, and A. Boyde, "Composite bounds on the elastic modulus of bone," Journal of biomechanics, vol. 41, no. 11, pp. 2585-2588, 2008.

[232] A. Knöller, T. Runčevski, R. E. Dinnebier, J. Bill, and Z. Burghard, "Cuttlebone-like v2o5 nanofibre scaffolds-advances in structuring cellular solids," Scientific Reports, vol. 7 , p. 42951, 2017.

[233] L. J. Gibson and M. F. Ashby, Cellular solids: structure and properties. Cambridge university press, 1999. 
[234] L. North, D. Labonte, M. Oyen, M. Coleman, H. Caliskan, and R. Johnston, "Interrelated chemical-microstructural-nanomechanical variations in the structural units of the cuttlebone of sepia officinalis," APL Materials, vol. 5, no. 11, p. 116103, 2017. 